International Journal of Bifurcation and Chaos, Vol. 10, No. 6 (2000) 1171-1266

(c) World Scientific Publishing Company

\title{
NEURAL EXCITABILITY, SPIKING AND BURSTING
}

\author{
EUGENE M. IZHIKEVICH* \\ The Neurosciences Institute, 10640 John Jay Hopkins Drive, \\ San Diego, CA 92121, USA \\ Center for Systems Science $\mathfrak{G}^{6}$ Engineering, Arizona State University, \\ Tempe, AZ 85287-7606, USA
}

Received June 9, 1999; Revised October 25, 1999

\begin{abstract}
Bifurcation mechanisms involved in the generation of action potentials (spikes) by neurons are reviewed here. We show how the type of bifurcation determines the neuro-computational properties of the cells. For example, when the rest state is near a saddle-node bifurcation, the cell can fire all-or-none spikes with an arbitrary low frequency, it has a well-defined threshold manifold, and it acts as an integrator; i.e. the higher the frequency of incoming pulses, the sooner it fires. In contrast, when the rest state is near an Andronov-Hopf bifurcation, the cell fires in a certain frequency range, its spikes are not all-or-none, it does not have a well-defined threshold manifold, it can fire in response to an inhibitory pulse, and it acts as a resonator; i.e. it responds preferentially to a certain (resonant) frequency of the input. Increasing the input frequency may actually delay or terminate its firing.

We also describe the phenomenon of neural bursting, and we use geometric bifurcation theory to extend the existing classification of bursters, including many new types. We discuss how the type of burster defines its neuro-computational properties, and we show that different bursters can interact, synchronize and process information differently.
\end{abstract}

\section{Introduction}

\subsection{Neurons}

The brain is made up of many types of cells, including neurons, neuroglia, and Schwann cells. The latter two types make up almost one-half of brain's volume, but neurons are believed to be the key elements in signal processing.

There are as many as $10^{11}$ neurons in the human brain, and each can have more than 10,000 synaptic connections with other neurons. Neurons are slow, unreliable analog units, yet working together they carry out highly sophisticated computations in cognition and control.

Action potentials play a crucial role among the many mechanisms for communication between neurons. They are abrupt changes in the electrical potential across a cell's membrane, see Fig. 1, and they can propagate in essentially constant shape away from the cell body along axons and toward synaptic connections with other cells.

The problems of propagation and transmission of neuronal signals are described elsewhere (see e.g. [Shepherd, 1983; Johnston \& Wu, 1995]). In this paper, we discuss mathematical aspects of the generation of action potentials.

\subsection{Why spiking?}

There is a common belief that action potentials are generated only by neurons and solely for the purpose of communication. However, many kinds of cells are known to generate voltage spikes across their cell membranes, including cells from the pumpkin stem, tadpole skin, and annelid eggs. Also, action potentials play certain roles in cell division, fertilization, morphogenesis, secretion of hormones, ion transfer, cell volume control, etc.

*E-mail: Eugene.Izhikevich@nsi.edu; URL: http://math.la.asu.edu/ eugene 

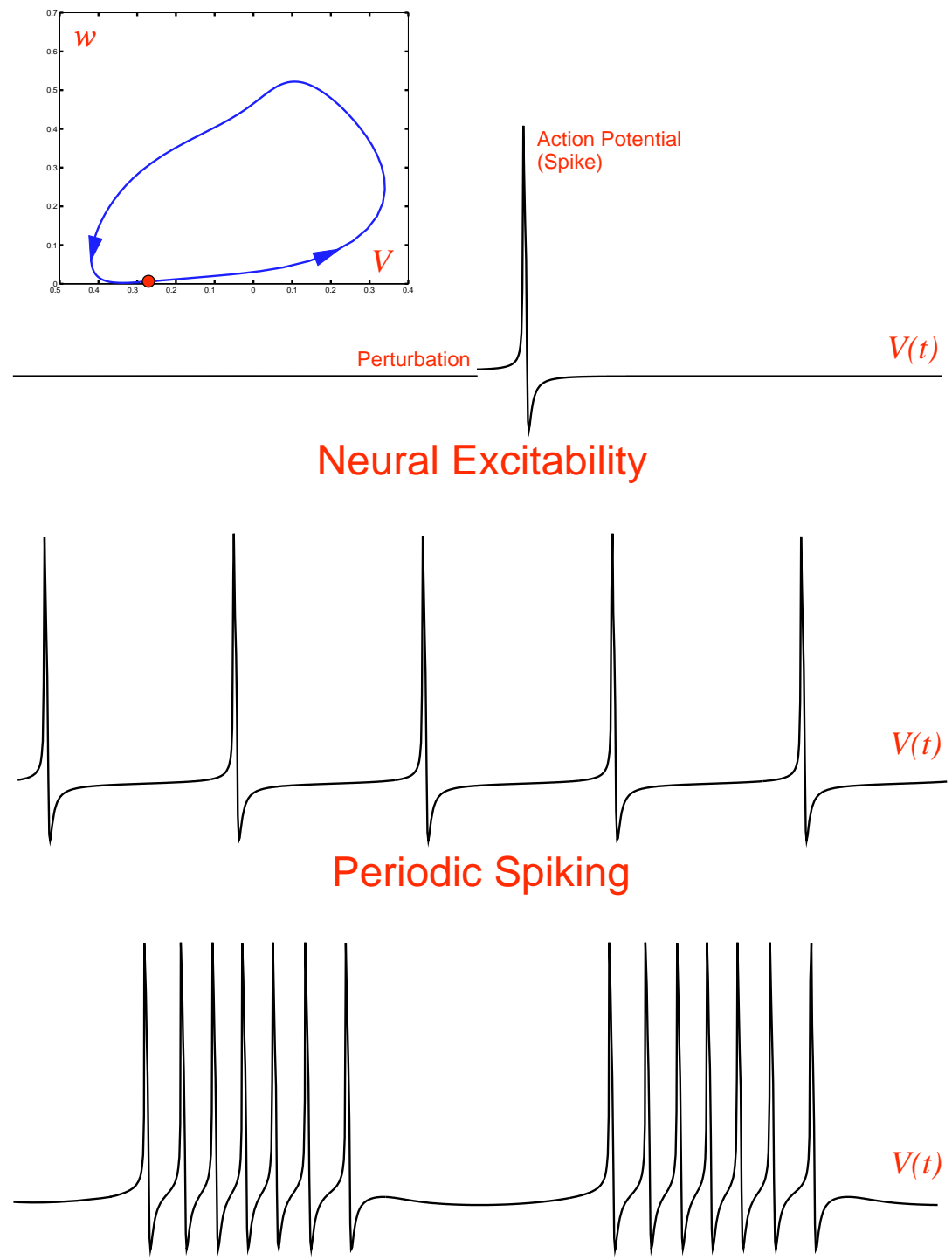

\section{Periodic Bursting}

Fig. 1. Examples of neural excitability, periodic spiking and bursting. (Shown are simulations of the Morris-Lecar [1981] model. A slow subsystem is added to obtain the bursting solution.)

[Shepherd, 1981, 1983], and might be irrelevant to cell-cell signaling. Nonetheless, our major goal in this tutorial paper is to review various spiking mechanisms in the context of neural signaling and information processing.

\subsection{Ionic mechanisms}

Action potentials are generated and sustained by ionic currents through the cell membrane. The ions most involved are sodium, $\mathrm{Na}^{+}$, calcium, $\mathrm{Ca}^{++}$, and potassium, $\mathrm{K}^{+}$. In the simplest case an increase in the membrane potential activates (opens) $\mathrm{Na}^{+}$ and/or $\mathrm{Ca}^{++}$channels, resulting in rapid inflow of the ions and further increase in the membrane potential. Such positive feedback leads to sudden and abrupt growth of the potential. This triggers a relatively slower process of inactivation (closing) of the channels and/or activation of $\mathrm{K}^{+}$channels, which leads to increased $\mathrm{K}^{+}$current and eventually reduces the membrane potential. These simplified positive and negative feedback mechanisms are responsible for the generation of action potentials.

There are more than a dozen of various ionic currents having divers activation and inactivation dynamics and occurring on disparate time 
scales [Llinas, 1988]. Almost any combination of them could result in interesting nonlinear behavior, such as neural excitability. Therefore, there could be thousands of different biophysically detailed conductance-based models. Neither of them is completely right or wrong.

\subsection{Dynamical mechanisms}

In this paper we view neurons from the perspective of dynamical systems, and we use geometrical methods to illustrate possible bifurcations and their role in the computational properties of neurons.

We say that a neuron is quiescent if its membrane potential is at rest or it exhibits small amplitude ("subthreshold") oscillations. In dynamical system terminology this corresponds to the system residing at an equilibrium or a small amplitude limit cycle attractor, respectively. A neuron is said to be excitable if a small perturbation away from a quiescent state can result in a large excursion of its potential before returning to quiescence. We will show that such large excursions exist because the quiescent state is near a bifurcation.

The neuron can fire spikes periodically when there is a large amplitude limit cycle attractor, which may coexist with the quiescent state. We also discuss briefly quasiperiodic and chaotic firing.

\subsection{Excitability}

The type of bifurcation the quiescent state experiences (Figs. 7, 29 and 30) determines the excitable properties of a cell, and hence its

Table 1. A summary of relevant codimension 1 bifurcations of quiescent state. Parameter $\lambda$ measures the distance to the bifurcation.

\begin{tabular}{|c|c|c|c|c|c|c|}
\hline \multirow{2}{*}{$\begin{array}{l}\text { Bifurcation of } \\
\text { Rest State }\end{array}$} & \multicolumn{4}{|c|}{ Initial Spiking } & \multirow{2}{*}{$\begin{array}{l}\text { Subthreshold } \\
\text { Oscillations }\end{array}$} & \multirow{2}{*}{$\begin{array}{l}\text { Reference } \\
\text { Figures }\end{array}$} \\
\hline & Behavior & Frequency & Amplitude & Operation & & \\
\hline Fold & bi-stable & nonzero & fixed & integrator & irrelevant & $7,9,21$ \\
\hline Saddle-node on & & & & & & \\
\hline Invariant Circle & excitable & zero $(\sqrt{\lambda})$ & fixed & integrator & irrelevant & $7,8,9,11$ \\
\hline Supercritical Hopf & excitable & nonzero & zero $(\sqrt{\lambda})$ & resonator & damped & 7 \\
\hline Subcritical Hopf & bi-stable & nonzero & arbitrary & resonator & damped & 7,16 \\
\hline $\begin{array}{l}\text { Bifurcation of } \\
\text { Subthreshold } \\
\text { Oscillation }\end{array}$ & & & & & $\begin{array}{l}\text { Subthreshold } \\
\text { Oscillation } \\
\text { Frequency }\end{array}$ & \\
\hline $\begin{array}{l}\text { Fold } \\
\text { Limit Cycle }\end{array}$ & bi-stable & nonzero & arbitrary & $\begin{array}{l}\text { integrator } \\
\text { resonator }\end{array}$ & nonzero & $\begin{array}{c}35 \\
29,31\end{array}$ \\
\hline $\begin{array}{l}\text { Saddle } \\
\text { Homoclinic Orbit }\end{array}$ & $\begin{array}{l}\text { bi-stable } \\
\text { excitable }\end{array}$ & nonzero & fixed & resonator & zero $(1 /|\ln \lambda|)$ & $29,31,34$ \\
\hline $\begin{array}{l}\text { Saddle-Focus } \\
\text { Homoclinic Orbit }\end{array}$ & $\begin{array}{l}\text { bi-stable } \\
\text { excitable }\end{array}$ & nonzero & fixed & resonator & zero $(1 /|\ln \lambda|)$ & 30 \\
\hline $\begin{array}{l}\text { Focus-Focus } \\
\text { Homoclinic Orbit }\end{array}$ & $\begin{array}{l}\text { bi-stable } \\
\text { excitable }\end{array}$ & nonzero & fixed & resonator & zero $(1 /|\ln \lambda|)$ & \\
\hline $\begin{array}{l}\text { Subcritical } \\
\text { Flip }\end{array}$ & bi-stable & nonzero & arbitrary & $\begin{array}{l}\text { integrator } \\
\text { resonator }\end{array}$ & nonzero & 30 \\
\hline Subcritical & & & & & & \\
\hline Neimark-Sacker & bi-stable & nonzero & arbitrary & resonator & nonzero & 30 \\
\hline Blue-sky Catastrophe & excitable & zero $(\sqrt{\lambda})$ & fixed & integrator & nonzero & 30 \\
\hline $\begin{array}{l}\text { Fold Limit Cycle } \\
\text { on Homoclinic Torus }\end{array}$ & excitable & zero $(\sqrt{\lambda})$ & fixed & integrator & nonzero & 30 \\
\hline
\end{tabular}


neuro-computational attributes. For example,

- When the rest state is near a saddle-node on invariant circle bifurcation, the neuron can fire allor-none spikes with an arbitrary low frequency, it has a well-defined threshold manifold, it can distinguish between excitatory and inhibitory input, and it acts as an integrator; i.e. the higher the frequency of incoming spikes, the sooner it fires.

- When the rest state is near an Andronov-Hopf bifurcation, the neuron fires in a certain frequency range, it does not have all-or-none spikes, it does not have a well-defined threshold manifold, it can fire in response to an inhibitory pulse, and it acts as a resonator; i.e. it responds preferentially to a certain (resonant) frequency of the input. Increasing the input frequency may actually delay or terminate its firing.

We discuss neural excitability in Sec. 2 and summarize some basic results in Table 1.

\subsection{Periodic spiking}

Neuro-computational properties of cells also depend on bifurcations of large amplitude limit cycles corresponding to periodic spiking. In general, such bifurcations differ from bifurcations of quiescent states: For example, when the limit cycle is about to disappear via a saddle homoclinic orbit, a fold limit cycle bifurcation, or it loses stability through a subcritical flip or Neimark-Sacker bifurcation, it coexists with a stable quiescent state. Hence a weak perturbation having appropriate timing can shut down periodic spiking prematurely. We discuss these and other issues in Sec. 3 and summarize some major results in Table 2.

\subsection{Bursting}

When neuron activity alternates between a quiescent state and repetitive spiking, the neuron activity is said to be bursting; see Fig. 1. It is usually caused by a slow voltage- or calcium-dependent process that can modulate fast spiking activity. There

Table 2. A summary of relevant codimension 1 bifurcations of large amplitude spiking. Parameter $\lambda$ measures the distance to the bifurcation.

\begin{tabular}{|c|c|c|c|c|}
\hline \multirow{2}{*}{$\begin{array}{l}\text { Bifurcation of } \\
\text { Periodic Firing }\end{array}$} & \multirow[b]{2}{*}{ Behavior } & \multicolumn{2}{|c|}{ Terminating Spiking } & \multirow[b]{2}{*}{ Locking } \\
\hline & & Frequency & Amplitude & \\
\hline Saddle-node on Invariant Circle & excitable & $\operatorname{zero}(\sqrt{\lambda})$ & fixed & difficult \\
\hline Supercritical Hopf & excitable & nonzero & zero $(\sqrt{\lambda})$ & easy \\
\hline Fold Limit Cycle & bi-stable & nonzero & arbitrary & easy \\
\hline Saddle Homoclinic Orbit & bi-stable & zero $(1 /|\ln \lambda|)$ & fixed & easy (?) \\
\hline Saddle-Focus Homoclinic Orbit & bi-stable & zero $(1 /|\ln \lambda|)$ & fixed & easy (?) \\
\hline Focus-Focus Homoclinic Orbit & bi-stable & zero $(1 /|\ln \lambda|)$ & fixed & easy (?) \\
\hline Subcritical Flip & bi-stable & nonzero & arbitrary & easy \\
\hline Subcritical Neimark-Sacker & bi-stable & nonzero & arbitrary & easy \\
\hline Blue-sky & excitable & zero $(\sqrt{\lambda})$ & fixed & difficult \\
\hline \multicolumn{5}{|l|}{$\begin{array}{l}\text { Bifurcation of } \\
\text { Quasi-periodic } \\
\text { Firing }\end{array}$} \\
\hline $\begin{array}{r}\text { Fold Limit Cycle on } \\
\text { Homoclinic Torus }\end{array}$ & excitable & zero $(\sqrt{\lambda})$ & fixed & difficult \\
\hline
\end{tabular}




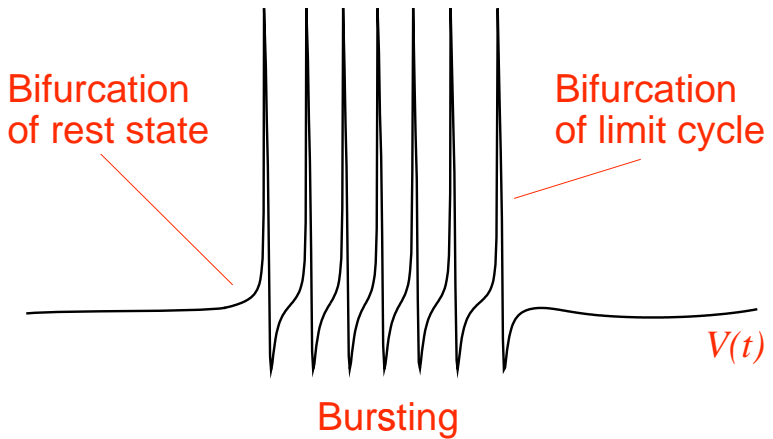

Fig. 2. Two important bifurcations associated with bursting.

are two important bifurcations (see Fig. 2) associated with bursting:

- Bifurcation of a quiescent state that leads to repetitive spiking; see left column in Table 3.

- Bifurcation of a spiking attractor that leads to quiescence; see top row in Table 3.

We restrict our consideration to bifurcations of codimension 1 , since they are the most probable to be encountered in nature.

We refer to a burster as being a point-cycle when the quiescent state is an equilibrium point and the spiking state is a limit cycle. When the quiescent state is a small amplitude (subthreshold) oscillation, then the burster is said to be cycle-cycle.

We refer to a burster as being planar when the fast spiking subsystem is two-dimensional. This imposes severe restriction on possible bifurcations. Table 3 summarizes 24 planar codimension 1 bursters. We name them after the two bifurcations involved. Among them are 16 point-cycle and 8 cycle-cycle bursters, which are in the upper and lower parts of the table, respectively. We discuss them in detail in Sec. 4.

A complete classification of all 16 planar pointcycle bursters, though without the naming scheme, was first provided by Hoppensteadt and Izhikevich [1997, Sec. 2.9]. Among them were the well-known

- "Fold/homoclinic" burster, also known as "square-wave" or Type I burster.

- "Circle/circle" burster, also known as "parabolic" or Type II burster.

- "SubHopf/fold cycle" burster, also known as "elliptic" or Type III burster.

- "Fold/fold cycle" burster, also known as Type IV burster.

- "Fold/Hopf" burster, also known as "tapered" or Type V burster.

- "Fold/circle" burster, also known as "triangular" burster.

Many other bursting types listed in Table 3 are new. We show in Sec. 4 that there could be many more bursters if the fast subsystem is multidimensional. They are classified in Table 4.

The history of formal classification of bursting starts from the seminal paper by Rinzel [1987], who contrasted the bifurcation mechanism of the "square-wave", "parabolic", and "elliptic" bursters. Then, Bertram et al. [1995] suggested to refer to

Table 3. A classification of codimension 1 planar fast-slow bursters. The upper (lower) part of the table corresponds to point-cycle (cycle-cycle) bursters. See also Table 4 and Figs. 53 and 126.

\begin{tabular}{|c|c|c|c|c|}
\hline Bifurcations & $\begin{array}{c}\text { Saddle-Node } \\
\text { on Invariant } \\
\text { Circle }\end{array}$ & $\begin{array}{c}\text { Saddle } \\
\text { Homoclinic } \\
\text { Orbit }\end{array}$ & $\begin{array}{c}\text { Supercritical } \\
\text { Andronov-Hopf }\end{array}$ & Fold Limit Cycle \\
\hline Fold & fold/circle & fold/homoclinic & fold/Hopf & fold/fold cycle \\
\hline $\begin{array}{l}\text { Saddle-Node on } \\
\text { Invariant Circle }\end{array}$ & circle/circle & circle/homoclinic & circle/Hopf & circle/fold cycle \\
\hline $\begin{array}{l}\text { Supercritical } \\
\text { Andronov-Hopf }\end{array}$ & Hopf/circle & Hopf/homoclinic & Hopf/Hopf & Hopf/fold cycle \\
\hline $\begin{array}{l}\text { Subcritical } \\
\text { Andronov-Hopf }\end{array}$ & subHopf/circle & subHopf/homoclinic & subHopf/Hopf & subHopf/fold cycle \\
\hline Fold Limit Cycle & fold cycle/circle & fold cycle/homoclinic & fold cycle/Hopf & fold cycle/fold cycle \\
\hline $\begin{array}{l}\text { Saddle Homoclinic } \\
\text { Orbit }\end{array}$ & homoclinic/circle & homoclinic/homoclinic & homoclinic/Hopf & homoclinic/fold cycle \\
\hline
\end{tabular}



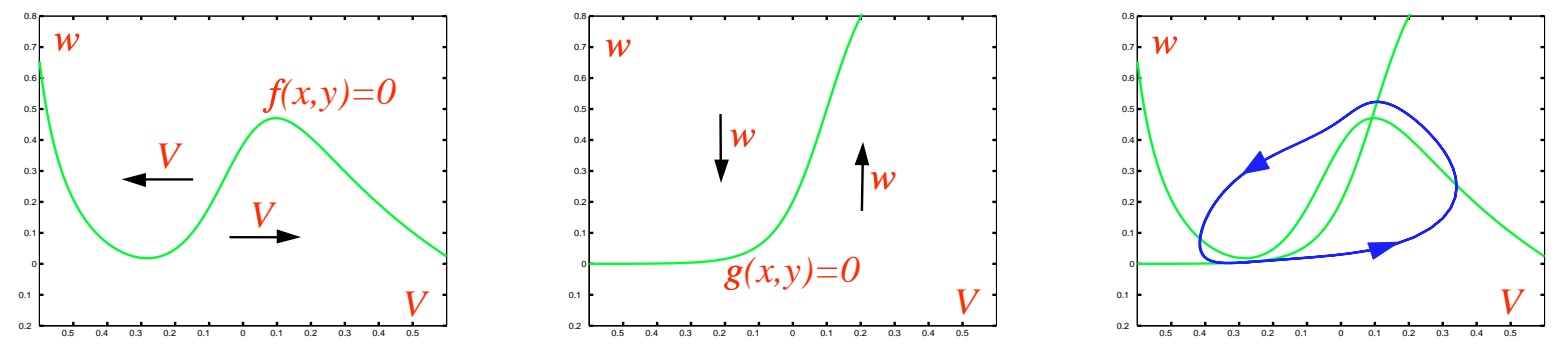

Fig. 3. Typical nullclines of planar neural systems. (Shown are nullclines for the Morris-Lecar [1981] model.)

the bursters using Roman numbers, and they added a new, Type IV type. Another, "tapered" type of bursting was studied simultaneously and independently by Holden and Erneux [1993a, 1993b], Smolen et al. [1993], and Pernarowski [1994]. Later de Vries [1998] suggested to refer to it as Type V burster. Yet another, "triangular" type of bursting was studied by Rush and Rinzel [1994], making the total number of identified bursters to be 6 . Their bifurcation mechanisms are summarized in Fig. 126.

There is a drastic difference between our approach to classification of bursting, and that of the scientists mentioned above. They use a bottom-up approach; that is, they consider biophysically plausible conductance-based models describing experimentally observable cellular behavior and then they try to determine the type of bursting these models exhibit. In contrast, we use the top-down approach: We consider all possible pairs of codimension 1 bifurcations of rest and spiking states, which result in different types of bursting, and then we invent a conductance-based model exhibiting each bursting type. Thus, many of our bursters are "theoretical" in the sense that they have yet to be seen in experiments. We return to this issue in Sec. 6.1.

\subsection{Nullclines and phase plane analysis}

We keep our exposition of bifurcations in neuron dynamics as general as possible. Although we use biophysically detailed Hodgkin-Huxley type neural models to illustrate many issues, most of our bifurcation diagrams and phase portraits are not related to any specific system of equations. Thus, we emphasize the essentials and omit irrelevant details.

Most of the bifurcations discussed here can be illustrated using a two-dimensional (planar) system of the form

$$
\begin{aligned}
\mu \dot{x} & =f(x, y) \\
\dot{y} & =g(x, y) .
\end{aligned}
$$

Much insight into the behavior of such systems can be gained by considering their nullclines, i.e. the sets determined by the conditions $f(x, y)=0$ or $g(x, y)=0$; see Fig. 3. When $0<\mu \ll 1$, nullclines are called fast and slow, respectively. Since the language of nullclines is universal in many areas of applied mathematics, we depict them (as green curves) in most illustrations.

\subsection{The canonical model approach}

Whenever possible, we use canonical models to illustrate neuron dynamics. Briefly, a model is canonical for a family of dynamical systems if every member of the family can be transformed into the model by a piecewise continuous possibly noninvertible change of variables; see Fig. 4. The definition of a canonical model generalizes the notions of topological normal form and versal unfolding, and it is discussed in detail in Chapter 4 in [Hoppensteadt \& Izhikevich, 1997], where one can find many examples of canonical models for neuroscience.

The advantage of considering a canonical model lies in its universality since the model provides information about behavior of the entire family. For example, the canonical model (2) describes the dynamics of any Class 1 excitable neuron regardless of the peculiarities of equations one chooses to model its activity. That is, if one modifies equations and adds more variables and parameters to take into account more ions, currents, pumps, etc., but the model still exhibits Class 1 excitability, then it still can be converted into the canonical model (2) by a (possibly different) change of variables. Thus, taking into account more biological data does not change the form of the canonical model (2), but 


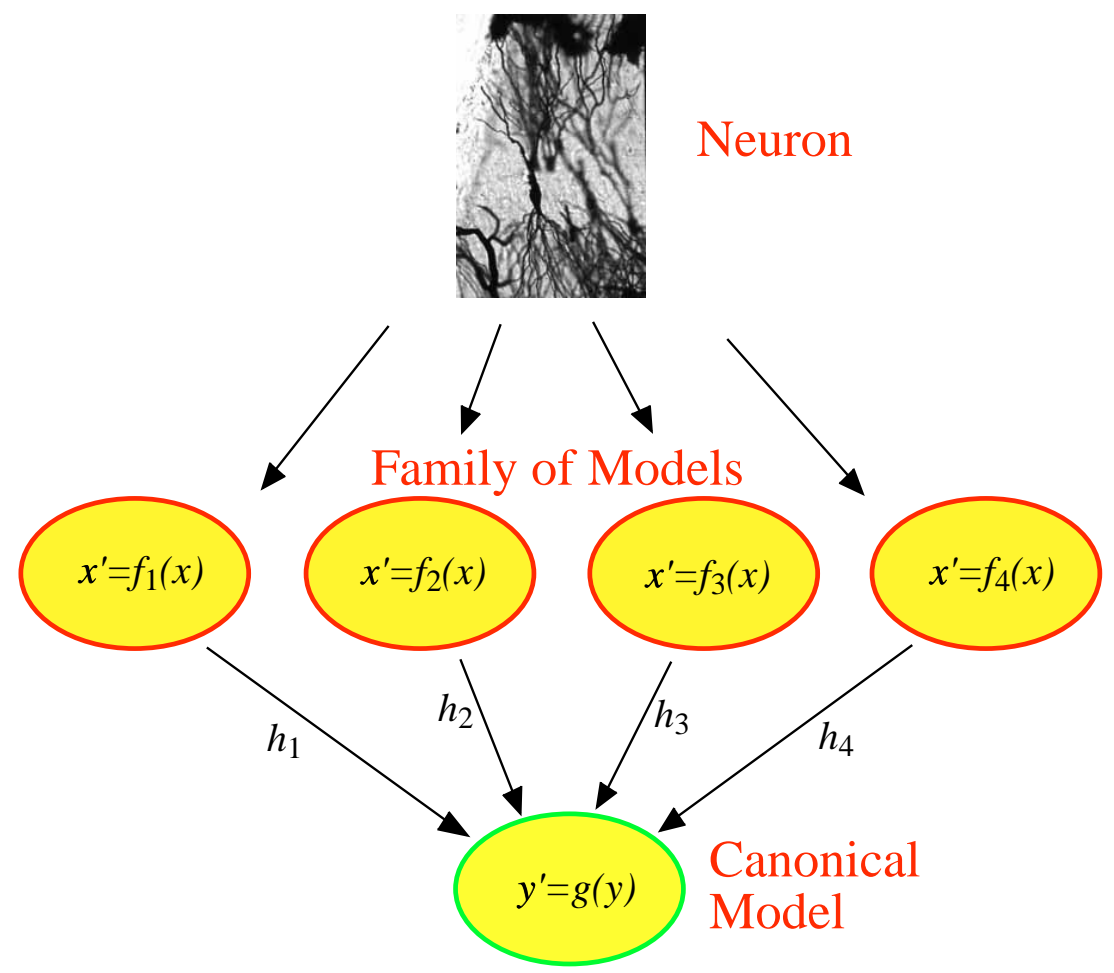

Fig. 4. A model $y^{\prime}=g(y)$ is canonical for the family of neural models, if each member of the family can be transformed into $y^{\prime}=g(y)$ by a piecewise continuous change of variables.

may only refine our knowledge of possible values of the parameter $r$ in the model.

\section{Neural Excitability}

Consider a system having a stable equilibrium that is a global attractor. According to the intuitive definition of excitability, small perturbations near the equilibrium can cause large excursions for the solution before it returns to the equilibrium. In dynamical system terminology this corresponds to a largeamplitude trajectory that starts and ends near the equilibrium; see Figs. 5 and 101. Such a trajectory is referred to as being a periodic pseudo-orbit, and the word "pseudo" is used to stress that the end points $x\left(t_{1}\right)$ and $x\left(t_{2}\right)$ are near but not equal to each other. If $x\left(t_{1}\right)=x\left(t_{2}\right)$, then the word "pseudo" should be dropped.

Thus, according to our definition, a dynamical system having a stable equilibrium is excitable if there is a large amplitude periodic pseudo-orbit passing near the equilibrium, as in Fig. 5. How large is "large" and how near is "near" depends on the context. Such periodic pseudo-orbit exists because the dynamical system is near a bifurcation. Indeed, a small perturbation of the vector field can cause the end points $x\left(t_{1}\right)$ and $x\left(t_{2}\right)$ to coalesce, thereby creating a periodic orbit, which corresponds to repetitive spiking. Thus, neurons are excitable because they are near a bifurcation (transition) from quiescence to repetitive firing.

Now suppose the equilibrium is not a global attractor, but has certain domain of attraction. Then we say that the system is excitable if the boundary of attraction is near the equilibrium. As in the previous case, small perturbations near the equilibrium can cause large excursions for the solution, but the solution does not return to the equilibrium. It is easy to see that such a neuron is also near a bifurcation, because a small perturbation of the vector field can cause the equilibrium to approach its boundary of attraction, resulting in loss of stability or disappearance.

Hodgkin's Classification of Excitability. A simple but useful criterion for classifying excitability was suggested by Hodgkin [1948]. He stimulated a cell by applying currents of various strengths. When the current is weak the cell is quiet. When the current is strong enough, the cell starts to fire repeatedly; see Fig. 6 . 

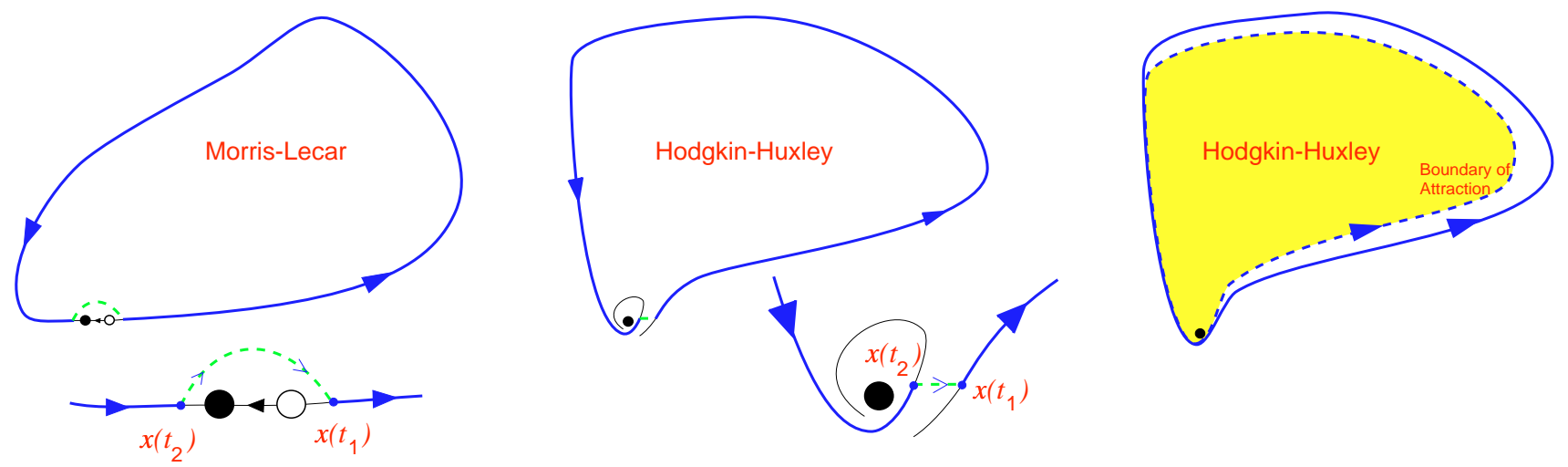

Fig. 5. A system is excitable when the rest state has a periodic pseudo-orbit (blue) or the boundary of attraction domain (yellow) is near the rest state. (A periodic pseudo-orbit is a piece of solution $x(t), t \in\left[t_{1} ; t_{2}\right]$ such that $x\left(t_{1}\right)$ is near $x\left(t_{2}\right)$.)

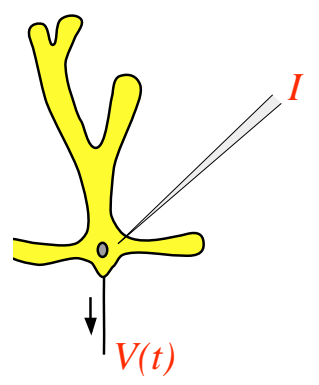

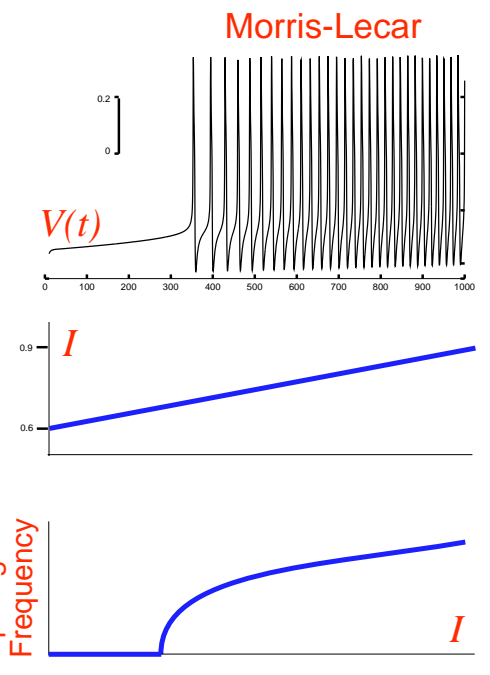

Class 1 Neural Excitability
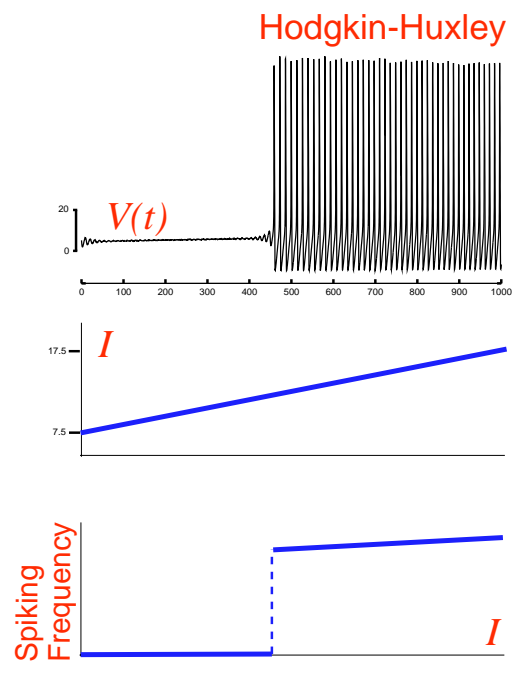

Class 2 Neural Excitability

Fig. 6. Transition from rest to repetitive spiking in two biophysical models when the strength of applied current, $I$, increases. (Noise is added to the Hodgkin-Huxley system to reduce the slow passage effect.)

He divided neurons into two classes according to the frequency of emerging firing:

- Class 1 neural excitability. Action potentials can be generated with arbitrarily low frequency. The frequency increases with increasing the applied current.

- Class 2 neural excitability. Action potentials are generated in a certain frequency band that is relatively insensitive to changes in the strength of the applied current.

Class 1 excitable neurons fire at frequencies that vary smoothly over a range of about $5-150 \mathrm{~Hz}$. The frequency band of the Class 2 excitable neurons is usually in the range $75-150 \mathrm{~Hz}$, but these bands can vary from neuron to neuron. The exact num- bers are not important to us here. The qualitative distinction between Class 1 and Class 2 excitable neurons is that the emerging oscillations have zero frequency in the former and nonzero frequency in the latter. This reflects different underlying bifurcation mechanisms.

Possible Bifurcations. The first attempt to classify excitability using dynamical systems belongs to FitzHugh [1955], although he did not use the bifurcation theory explicitly. In what follows we use the approach suggested by Rinzel and Ermentrout [1989] and consider the strength of applied current in Hodgkin's experiments as being a quasistatic bifurcation parameter. When the current increases, 

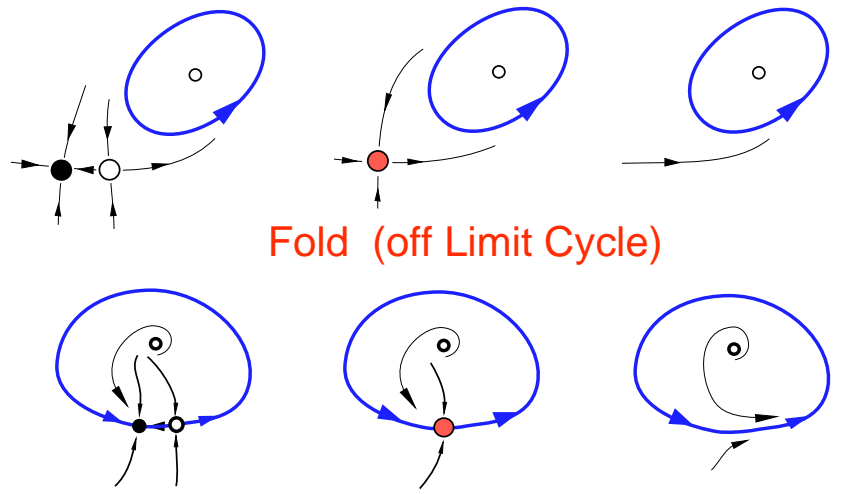

Saddle-Node on Invariant Circle
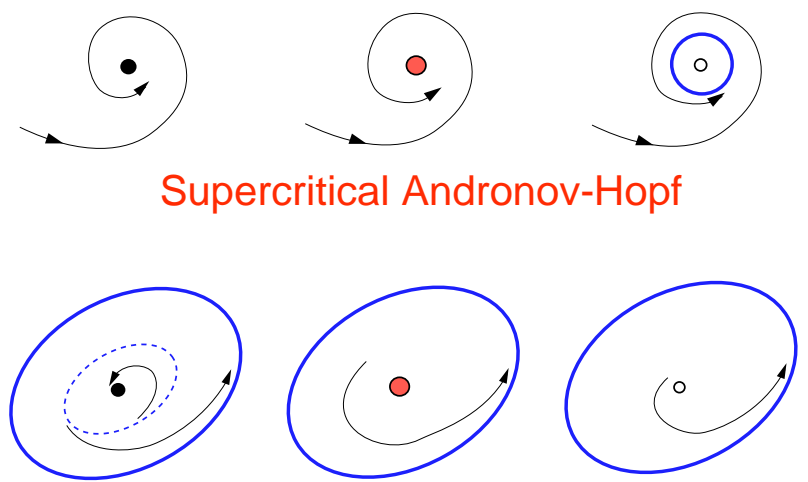

Subcritical Andronov-Hopf

Fig. 7. Codimension 1 bifurcations corresponding to a transition from equilibrium to oscillatory dynamics. Fold bifurcation occurs when the Jacobian matrix at the equilibrium has a zero eigenvalue. We refer to it as the saddle-node on invariant circle bifurcation when the center manifold makes a loop. Andronov-Hopf bifurcation occurs when the matrix has a pair of complex-conjugate eigenvalues with zero real part; see [Hoppensteadt \& Izhikevich, 1997] or [Kuznetsov, 1995] for complete definitions.

the rest potential increases until a bifurcation occurs, resulting in loss of stability or disappearance of the rest potential, and the neuron activity becomes oscillatory. The bifurcation resulting in transition from a quiescent to an oscillatory state determines the class of neural excitability.

Since there is an infinite number of possible bifurcations of an equilibrium, we consider only bifurcations of codimension 1 . Those corresponding to transition from an equilibrium to a limit cycle are summarized in Fig. 7.

We will see that

- Class 1 neural excitability is observed when a rest potential disappears through a saddle-node bifurcation on an invariant circle.

- Class 2 neural excitability is observed when a rest potential loses stability via an Andronov-Hopf bifurcation.

Is it not amazing that there are only two different dynamical (bifurcation) mechanisms for neural excitability despite the overwhelming number of ionic mechanisms? Another amazing fact is that Hodgkin's classification was largely ignored by the computational neuroscience community until the seminal publication by Rinzel and Ermentrout [1989].

The classification above is not perfect. For example, the fold (off a stable limit cycle) bifurcation can result in Class 1 or Class 2 excitability depending on the frequency of the existing limit cycle. If the system is near a codimension 2 saddle-node separatrix loop bifurcation (see Fig. 21), then the limit cycle is of low frequency. Similarly, the stable limit cycle in the subcritical Andronov-Hopf bifurcation may have low frequency when the system is near a certain codimension 2 bifurcation, which would appear to be Class 1 excitability. One may argue that we should disregard these cases because they have larger codimension and hence are not likely to be encountered in nature. However, bifurcations of codimension 2 do play important roles in neural bursting, and evolution may have developed separate physiological mechanisms to keep cell dynamics near such bifurcations.

\subsection{Class 1 excitable systems}

Let us consider a saddle-node on invariant circle bifurcation, which always results in Class 1 excitability with a typical periodic pseudo-orbit depicted in Fig. 5. Such a bifurcation is exhibited by the Wilson-Cowan model [Hoppensteadt \& Izhikevich, 1997], the VCON model [Hoppensteadt, 1997], the Morris-Lecar, Connor models [Ermentrout, 1996], the Traub model [Traub \& Miles, 1991], the Bower model [Wilson \& Bower, 1989], and many other biophysically detailed models. It is ubiquitous in twodimensional relaxation systems

$$
\begin{aligned}
\mu \dot{x} & =f(x, y) \\
\dot{y} & =g(x, y)
\end{aligned} \quad x, y \in \mathbb{R}, \mu \ll 1,
$$

having nullclines intersecting as shown in Fig. 8.

It is widely believed that the original HodgkinHuxley [1952] model does not exhibit Class 1 excitability unless the potassium $A$-current is added [Connor \& Stevens, 1971]. Rush and Rinzel [1995] have shown recently that $A$-current is not necessary 

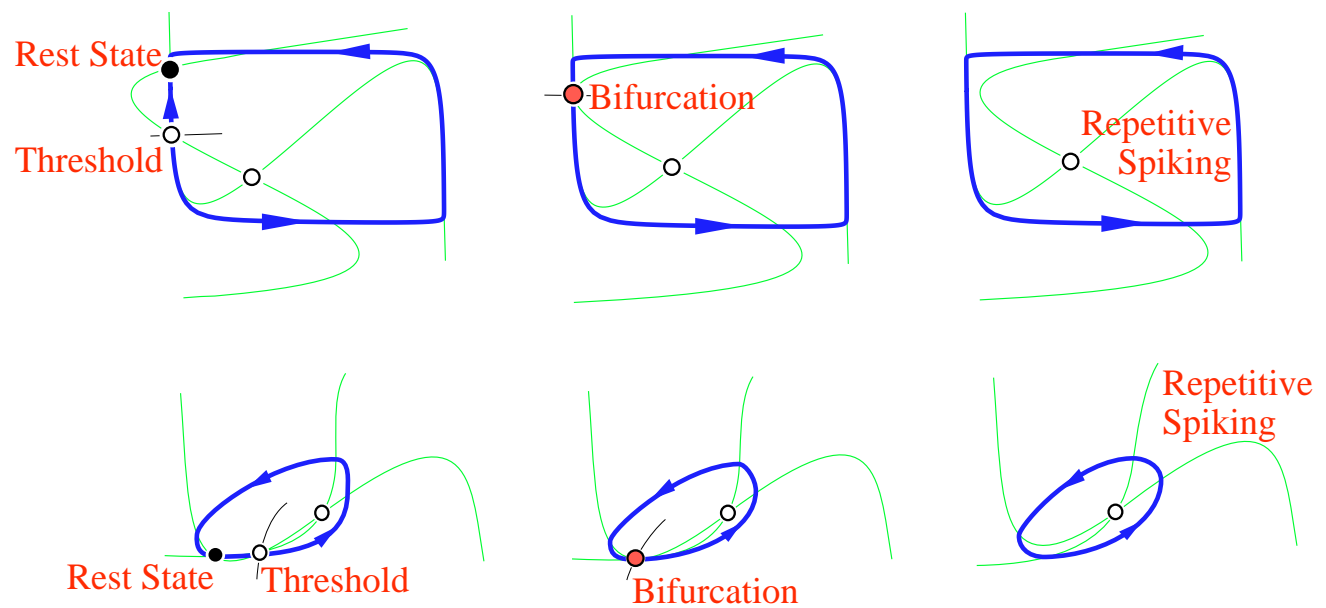

Fig. 8. Saddle-node on invariant circle bifurcation in two-dimensional relaxation systems (from [Hoppensteadt \& Izhikevich, 1997].

for Class 1 excitability if the sodium and potassium (in)activation curves in the Hodgkin-Huxley model are shifted appropriately.

\subsubsection{Threshold}

Whenever a bifurcation involves a saddle, the system has a well-defined threshold - the stable manifold of the saddle [FitzHugh, 1955], which is often referred to as a separatrix since it separates into two regions of the phase space having different qualitative behavior. Indeed, small perturbations of the solution that do not lead beyond the separatrix in Fig. 9 decay, while those crossing it grow away exponentially thereby producing a spike. Such a system is said to have all-or-none behavior. Because the solution eventually returns to the stable node (rest point), the system is not oscillatory, but excitable.

Notice that the threshold is a codimension 1 manifold, which can be a point only when the system is one-dimensional. Thus, it is futile to seek a threshold value of the membrane voltage, unless there is a way to freeze all the other variables.

\subsubsection{Canonical model for Class 1 excitable systems}

When the saddle and node in Fig. 8 coalesce and disappear, the vector field remains small at the location of the saddle-node point. Therefore, the solution $(x(t), y(t))$ spends most time near that point, then makes a relatively fast excursion, or spike. This observation lays the basis for proving the Ermentrout-Kopell Theorem for Class 1

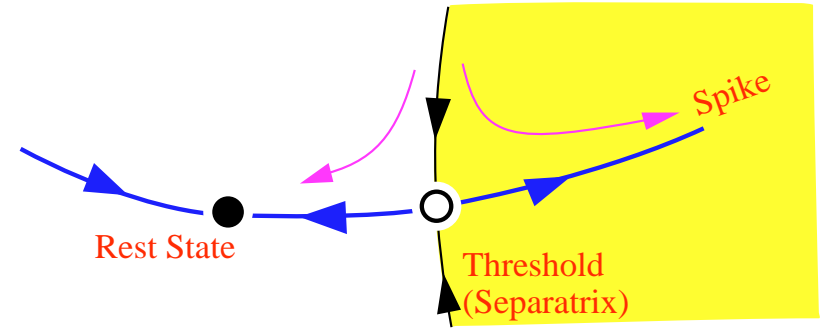

Fig. 9. Excitable behavior and a threshold at fold (saddlenode) bifurcation.

Neural Excitability [Hoppensteadt \& Izhikevich, 1997, Theorem 8.3].

Theorem 1. (Ermentrout-Kopell). A family of dynamical systems of the form

$$
\dot{x}=f(x, \lambda), \quad x \in R^{m}, \lambda \in \mathbb{R},
$$

having a saddle-node on invariant circle bifurcation for $\lambda=0$ has a nonlocal canonical model

$$
\vartheta^{\prime}=(1-\cos \vartheta)+(1+\cos \vartheta) r,
$$

plus higher-order terms in $\lambda$, where ${ }^{\prime}=d / d \tau, \tau=$ $\sqrt{|\lambda|} t$ is slow time, $\vartheta \in \mathbb{S}^{1}$ is a canonical variable along the invariant circle, and $r \in \mathbb{R}$ is some parameter that depends on $f$ and $\lambda$. That is, there is an open $\mathcal{O}(1)$-neighborhood, $W$, of the invariant circle and a mapping $h: W \rightarrow \mathbb{S}^{1}$ that projects all solutions of (1) to those of (2).

The mapping $h: W \rightarrow \mathbb{S}^{1}$ blows up a small neighborhood of the saddle-node point and compresses the entire invariant circle to an open set 


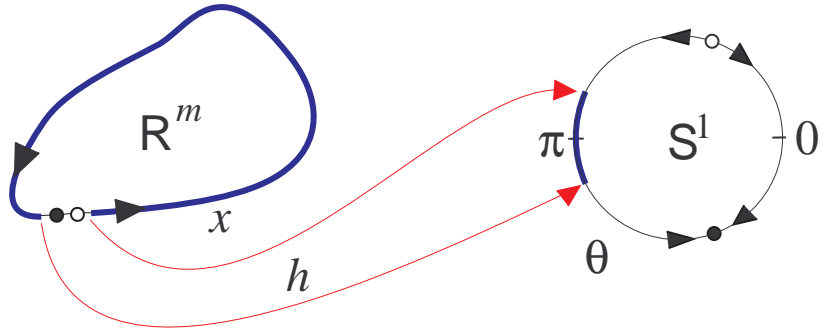

Fig. 10. The transformation $h$ maps solutions of (1) to those of (2) (from [Izhikevich, 1999b]).

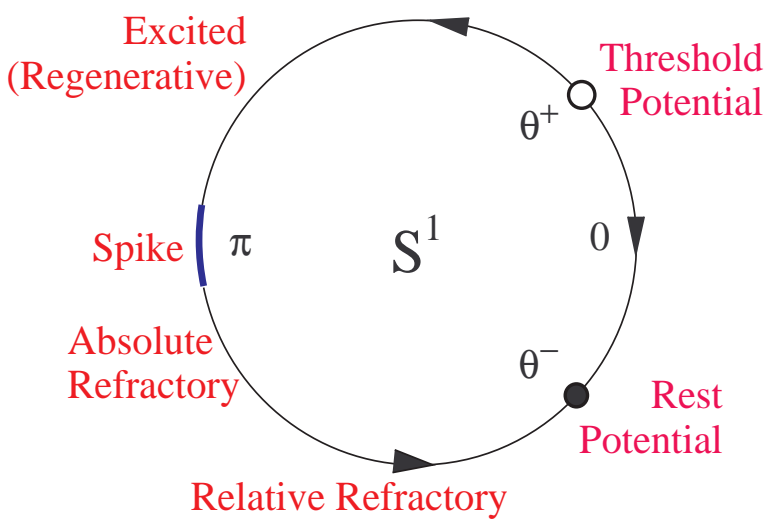

Fig. 11. Physiological diagram of the canonical model (2) for Class 1 neural excitability (from [Hoppensteadt \& Izhikevich, 1997]).

around $\pi \in \mathbb{S}^{1}$; see Fig. 10. Thus, when $x$ makes a rotation around the invariant circle (generates a spike), the canonical variable $\vartheta$ crosses a tiny open set around $\pi$.

The canonical model (2) has a simple behavior:

- If $r<0$, then there are two equilibria

$$
\vartheta^{ \pm}= \pm \cos ^{-1} \frac{1+r}{1-r}
$$

which are the rest and threshold states; see Fig. 11. The system is excitable in the following sense: Small perturbations of $\vartheta$ that do not lead beyond the threshold value $\vartheta^{+}$die out exponentially; in contrast, if $\vartheta$ is perturbed beyond the threshold, it grows further, passes the spike value $\vartheta=\pi$, and only after that returns to the rest state $\vartheta^{-}$. The equilibria $\vartheta^{-}$and $\vartheta^{+}$coalesce when $r \rightarrow 0$.

- If $r>0$, then there are no equilibria, and $\vartheta(t)$ oscillates with the frequency $\omega=2 \sqrt{r}$. Hence the original system (1) oscillates with the frequency $2 \sqrt{|\lambda| r}$, which has been confirmed by experimen- tal observations (see [Ermentrout, 1996; Guckenheimer et al., 1997]).

We see that $r$ plays the role of a bifurcation parameter in (2). When it crosses 0 , the qualitative behavior changes. If $r \neq 0$, then we can use the change of variables [Izhikevich, 1999b]

$$
\varphi=2 \operatorname{atan} \frac{1}{\sqrt{|r|}} \tan \frac{\vartheta}{2}
$$

to transform the canonical model into one of the following simple forms

$$
\begin{array}{ll}
\varphi^{\prime}=-\omega \cos \varphi & (\text { excitable activity; } r<0) \\
\varphi^{\prime}=\omega & \text { (periodic activity; } r>0)
\end{array}
$$

where $\omega=2 \sqrt{|r|}$ is a positive parameter. The transformation (3) justifies the empirical observation that the behavior of the canonical model for negative $r$ is equivalent to that for $r=-1$; and for positive $r$ is equivalent to that for $r=+1$.

\subsubsection{Slow adaptation currents}

While deriving the canonical model (2) we implicitly assume that all ionic processes in (1) occur on the time scale much faster than the interspike interval. To take into account slowly (in-)activating ionic currents, we consider the system

$$
\begin{aligned}
& \dot{x}=f(x, y, \lambda) \\
& \dot{y}=\mu g(x, y) .
\end{aligned}
$$

If $\mu=\mathcal{O}(|\lambda|)$, then this system is not near a saddlenode on invariant circle bifurcation, but near some other bifurcation of large codimension. Nevertheless, if dynamics of $y$ satisfy some fairly general and biophysically plausible conditions, such as $y=0$ is an exponentially stable equilibrium when $x$ is quiescent, then one can derive the canonical model

$$
\begin{aligned}
& \vartheta^{\prime}=(1-\cos \vartheta)+(1+\cos \vartheta)(r+s u) \\
& u^{\prime}=\delta(\vartheta-\pi)-\eta u
\end{aligned}
$$

where $\eta \ll 1$, and $\delta$ is the Dirac delta function. Whenever $\vartheta$ crosses $\pi$ (fires a spike), the slow variable $u$ experiences a step-like increase, then it slowly relaxes to $u=0$. The sign of $s$ determines whether this firing advances or delays the next firing, which results in spike facilitation or adaptation, respectively. We discuss the latter phenomenon in Sec. 3.1. 


\subsubsection{Weakly connected networks}

The canonical model (2) is probably the simplest excitable system in mathematical neuroscience: It is one-dimensional, ${ }^{1}$ it has Class 1 neural excitability or periodic activity, and it is biologically plausible in the sense that any other Class 1 excitable neuro-system can be converted to the form (2) by an appropriate change of variables. It is not surprising that it can be generalized [Hoppensteadt \& Izhikevich, 1997; Izhikevich, 1999b] to a weakly connected network of Class 1 excitable neurons

$$
\dot{x}_{i}=f_{i}\left(x_{i}, \lambda\right)+\varepsilon \sum_{j=1}^{n} g_{i j}\left(x_{i}, x_{j}, \lambda, \varepsilon\right)
$$

where $\varepsilon \ll 1$ measures the strength of connections.

Theorem 2. Suppose the system (6) satisfies the following two conditions

- Each subsystem

$$
\dot{x}_{i}=f_{i}\left(x_{i}, \lambda\right)
$$

has a saddle-node bifurcation on an invariant circle for $\lambda=0$.

- Each function $g_{i j}=0$ when $x_{j}$ is in some small neighborhood of the rest state.

Then there is an $\varepsilon_{0}>0$ such that for all $\varepsilon \ll \varepsilon_{0}$ the family (6) has one of the following canonical models depending on the relative magnitudes of $|\lambda|$ and $\varepsilon$ (see Fig. 12).

Case 1. $|\lambda| \ll \varepsilon^{2}$.

$$
\begin{aligned}
\vartheta_{i}^{\prime}= & \left(1-\cos \vartheta_{i}\right)+\left(1+\cos \vartheta_{i}\right) r_{i} \\
& +\sum_{j=1}^{n} w_{i j}\left(\vartheta_{i}\right) \delta\left(\vartheta_{j}-\pi\right)
\end{aligned}
$$

where $\delta$ is the Dirac delta function, each function $w_{i j}$ has the form

$$
w_{i j}\left(\vartheta_{i}\right)=2 \operatorname{atan}\left(\tan \frac{\vartheta_{i}}{2}+s_{i j}\right)-\vartheta_{i},
$$

see Fig. 13, and each $s_{i j}$ is a constant.

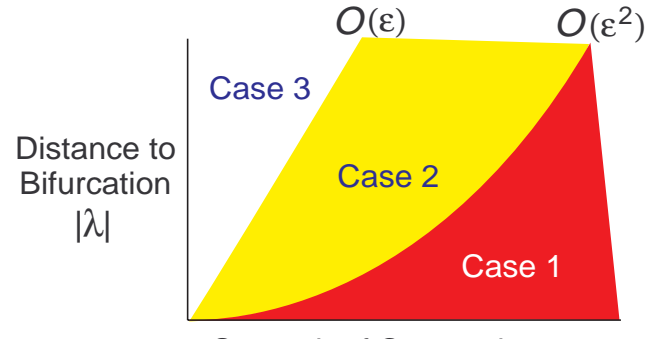

Strength of Connections $\varepsilon$

Fig. 12. A weakly connected network of Class 1 excitable neurons (6) has various canonical models depending on the relative sizes of $\lambda$ and $\varepsilon$ (from [Izhikevich, 1999b]).

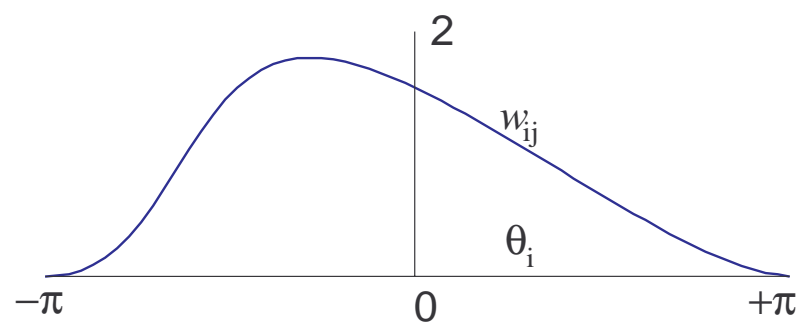

Fig. 13. A typical graph of the function (9). (Here $s_{i j}=1$. From [Izhikevich, 1999b].)

Case 2. $\varepsilon^{2} \ll|\lambda| \ll \varepsilon$.

$\vartheta_{i}^{\prime}=\left(1-\cos \vartheta_{i}\right)+\left(1+\cos \vartheta_{i}\right)\left(r_{i}+\sum_{j=1}^{n} s_{i j} \delta\left(\vartheta_{j}-\pi\right)\right)$

Case 3. $|\lambda| \gg \varepsilon$ and subsystems (7) have limit cycles with equal frequency.

$$
\varphi_{i}^{\prime}=\omega_{i}+\sum_{j=1}^{n} s_{i j} H\left(\varphi_{j}-\varphi_{i}\right), \quad H(\chi)=1-\cos \chi,
$$

The canonical phase model (11) differs from the Kuramoto model, which has $H(\chi)=\sin \chi$.

Systems (9) and (10) are pulse-coupled neural networks in the sense that they are uncoupled unless at least one $\vartheta_{j}$ crosses $\pi$; i.e. it fires a spike. This event produces a step-like increase in other variables due to the term containing Dirac's delta function. Unlike the standard integrate-and-fire model, the magnitude of the pulse is not a constant, but it depends on the current state of the post-synaptic neuron.

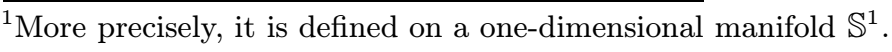




\subsubsection{Slowly connected networks}

When we consider weakly connected systems of the form (6) we implicitly assume that synaptic transmission is relatively fast in comparison with the interspike period. To study the case of slow transmission, we consider the system of the form

$$
\begin{aligned}
& \dot{x}_{i}=f_{i}\left(x_{i}, \lambda\right)+\varepsilon \sum_{j=1}^{n} g_{i j}\left(x_{i}, y_{j}\right) \\
& \dot{y}_{i}=\mu p_{i}\left(x_{i}, y_{i}\right)
\end{aligned}
$$

where the vector $y_{i}$ describes slow synaptic processes. We require that $y_{i}=0$ be an exponentially stable equilibrium when $x_{i}$ is quiescent. The canonical model for the system above is derived elsewhere, and it has the form

$$
\begin{aligned}
& \vartheta_{i}^{\prime}=\left(1-\cos \vartheta_{i}\right)+\left(1+\cos \vartheta_{i}\right)\left(r_{i}+\sum_{j=1}^{n} s_{i j} w_{j}\right) \\
& w_{i}^{\prime}=\delta\left(\vartheta_{j}-\pi\right)-\eta w_{i}
\end{aligned}
$$

where $\eta=\mathcal{O}(\mu / \sqrt{|\lambda|})$. The term $s_{i i} w_{i}$ denotes not a self-synapse, but a slow adaptation $\left(s_{i i}<0\right)$ or facilitation $\left(s_{i i}>0\right)$ process.

A remarkable fact is that $\varepsilon$ does not have to be small for the derivation to be valid. This collaborates the well-known principle that strongly but slowly connected systems are similar to weakly connected systems in many respects [Frankel \& Kiemel, 1993].

\subsubsection{Class 1 excitable neurons are integrators}

Consider the canonical model (8) or (10) and suppose that $s_{i j}>0$, which corresponds to excitatory

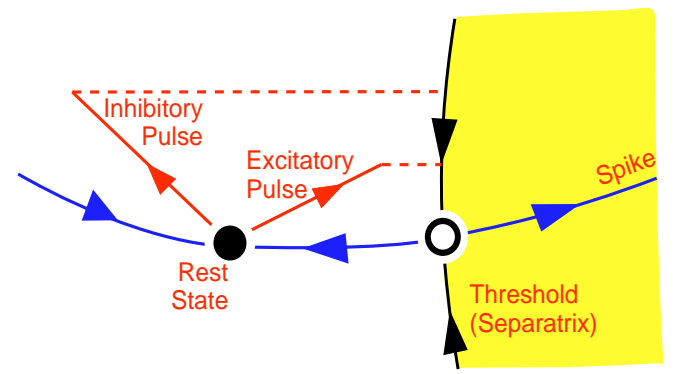

Class 1 Excitable Neuron synapse. Then both $w_{i j}\left(\vartheta_{i}\right)$ and $\left(1+\cos \vartheta_{i}\right)$ are non-negative for any $\vartheta_{i}$. Thus, each incoming spike advances $\vartheta_{i}$ toward $\pi$. The higher the frequency of incoming spikes, the sooner $\vartheta_{i}$ will reach $\pi$ and "fire". This important "integrate-and-fire" feature makes Class 1 excitable systems integrators. In contrast, we will show that Class 2 excitable systems may act as resonators; i.e. they respond preferentially to certain resonant frequencies of the input.

\subsubsection{Post-inhibitory spikes}

Class 1 excitatory neurons never fire in response to weak inhibitory pulse. Indeed, from Fig. 14 we see that weak excitatory (inhibitory) pulses decrease (increase) the distance to the threshold manifold. This is not valid for Class 2 excitable neurons as we discuss below.

Nevertheless, a strong inhibitory input could make Class 1 neurons fire, e.g. when the threshold manifold curves around the rest state as we illustrate in Fig. 15. Similarly, prolonged inhibitory input could elicit action potentials too via activating slow subthreshold inward currents, such as the $h$-current. Thus, Class 1 excitatory neurons could exhibit post-inhibitory spikes, but only when inhibition is either strong or prolonged.

\subsubsection{Synchronization}

Identical Class 1 excitable neurons are difficult to synchronize. This was shown numerically by [Hansel et al., 1995], and analytically by Ermentrout [1996] for a pair of neurons. Later this result was extended to networks of arbitrary number of neurons [Izhikevich, 1999b].

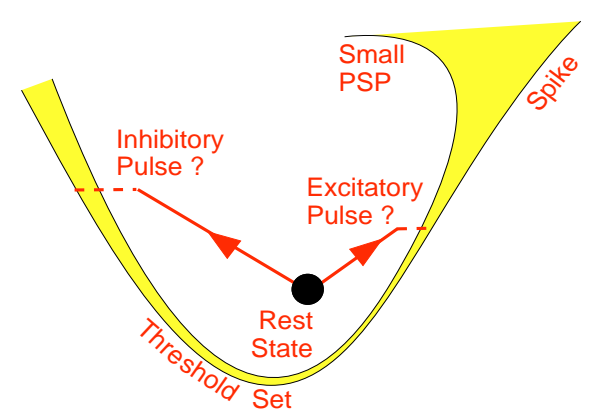

Class 2 Excitable Neuron

Fig. 14. Class 1 excitatory neurons: Excitatory (inhibitory) pulses decrease (increase) the distance to the threshold manifold (red dotted line). Class 2 excitatory neurons: Both excitatory and inhibitory pulses decrease the distance to the threshold set, thereby making the distinction between excitation and inhibition a bit confusing. 


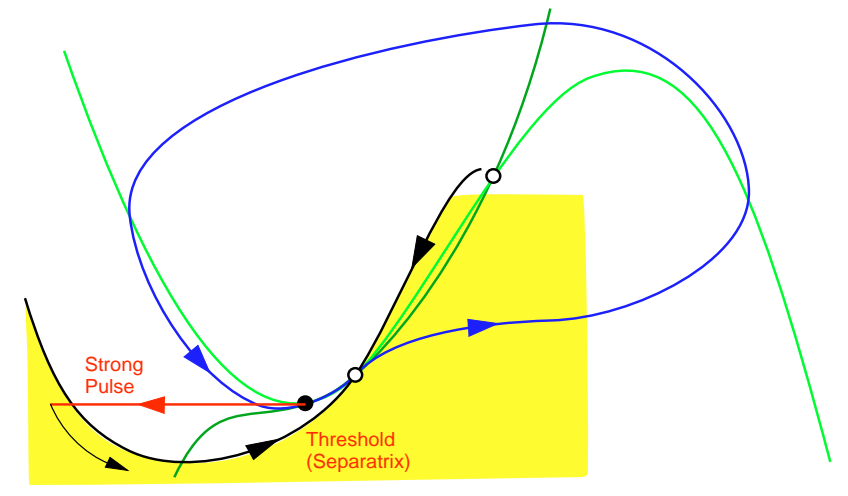

Fig. 15. A strong inhibitory input can elicit spike in Class 1 excitable neuron when the threshold manifold curves around the rest state.

Indeed, consider the phase model (11) and suppose that $\omega_{1}=\omega_{2}$ and $s_{12}=s_{21}=1$. Then, the phase difference, $\chi=\varphi_{2}-\varphi_{1}$, satisfies

$$
\chi^{\prime}=H(-\chi)-H(\chi) \equiv 0 .
$$

because $H(\chi)=1-\cos \chi$ is an even function. This prevents stable synchronization, at least on the time scale of order $1 / \varepsilon$. In contrast, Class 2 excitable systems near supercritical Andronov-Hopf bifurcation have

$$
H(\chi)=\sin (\chi-\psi),
$$

where $\psi \in \mathbb{S}^{1}$ is some parameter that has the meaning of the natural phase difference [Hoppensteadt \& Izhikevich, 1996] because $\chi \rightarrow \psi$ in the unidirectional case. If $\psi \neq \pm \pi / 2$, then such systems always synchronize stably. Whether or not this fact can be extended to all Class 2 excitable and spiking systems is still unknown, although numerous simulations suggest so.

\subsection{Class 2 excitable systems near an Andronov-Hopf bifurcation}

The Andronov-Hopf bifurcation was thought to be the primary bifurcation of the rest potential in neurons because it is a primary route from rest to oscillations in the Hodgkin-Huxley model, which is one of the most significant models in computational neuroscience. As a result, this bifurcation has been scrutinized numerically and analytically by many researches (see e.g. [Hassard, 1978; Troy, 1978; Rinzel \& Miller, 1980; Hassard et al., 1981; Holden et al., 1991; Bedrov et al., 1992]).

A remarkable historical fact is that many important neuroscience properties, such as all-or-none response, threshold, and integration, have been introduced or illustrated using classical HodgkinHuxley model despite the fact that the model does not exhibit any of these properties, as we see below.

The Hodgkin-Huxley model, as well as many other biophysical models, has a typical bifurcation structure as depicted in Fig. 16. While the bifurcation parameter $I$ increases, stable and unstable limit cycles appear via fold limit cycle bifurcation. The latter shrinks down to the rest state and makes it lose stability via subcritical Andronov-Hopf bifurcation.

At any value of $I$ the phase portrait is equivalent to that of the topological normal form for a Bautin bifurcation [Kuznetsov, 1995; Izhikevich, 2000a]

$$
z^{\prime}=(a+\mathrm{i} \omega)+b z|z|^{2}-z|z|^{4}, \quad z \in \mathbb{C},
$$

Apart from qualitative illustration of bifurcations in Hodgkin-Huxley-type systems, the model above may be of limited value since it fails to reflect the relaxation nature of the dynamics.

\subsubsection{Threshold, excitability, and bistability}

An important consequence of relaxation dynamics is that the flow can undergo large contractions and expansions. For example, the stable and unstable limit cycles in Fig. 16(b) are so close to each other when they are near an equilibrium, that they could become indistinguishable if small noise is introduced into the system.

Figures 16(b) and 16(c) illustrate the bistable nature of dynamics of the Hodgkin-Huxley model. The unstable limit cycle separates the basins of attraction of the rest state and the large amplitude limit cycle corresponding to repetitive spiking. Therefore, the unstable cycle is a threshold manifold.

When the equilibrium is a global attractor, as in Fig. 16(a), a small perturbation of a solution near the equilibrium can still produce a large deviation; see Fig. 17. Therefore, the system is excitable with a typical periodic pseudo-orbit depicted in Fig. 5. However, a threshold manifold may not exist. Indeed, if the initial condition lies in the yellow region between the two solutions in the right inlet in Fig. 17, the system can produce a spike having an arbitrary intermediate amplitude. We refer to the region as being a threshold set. We see that the Hodgkin-Huxley model does not have all-or-none response. 

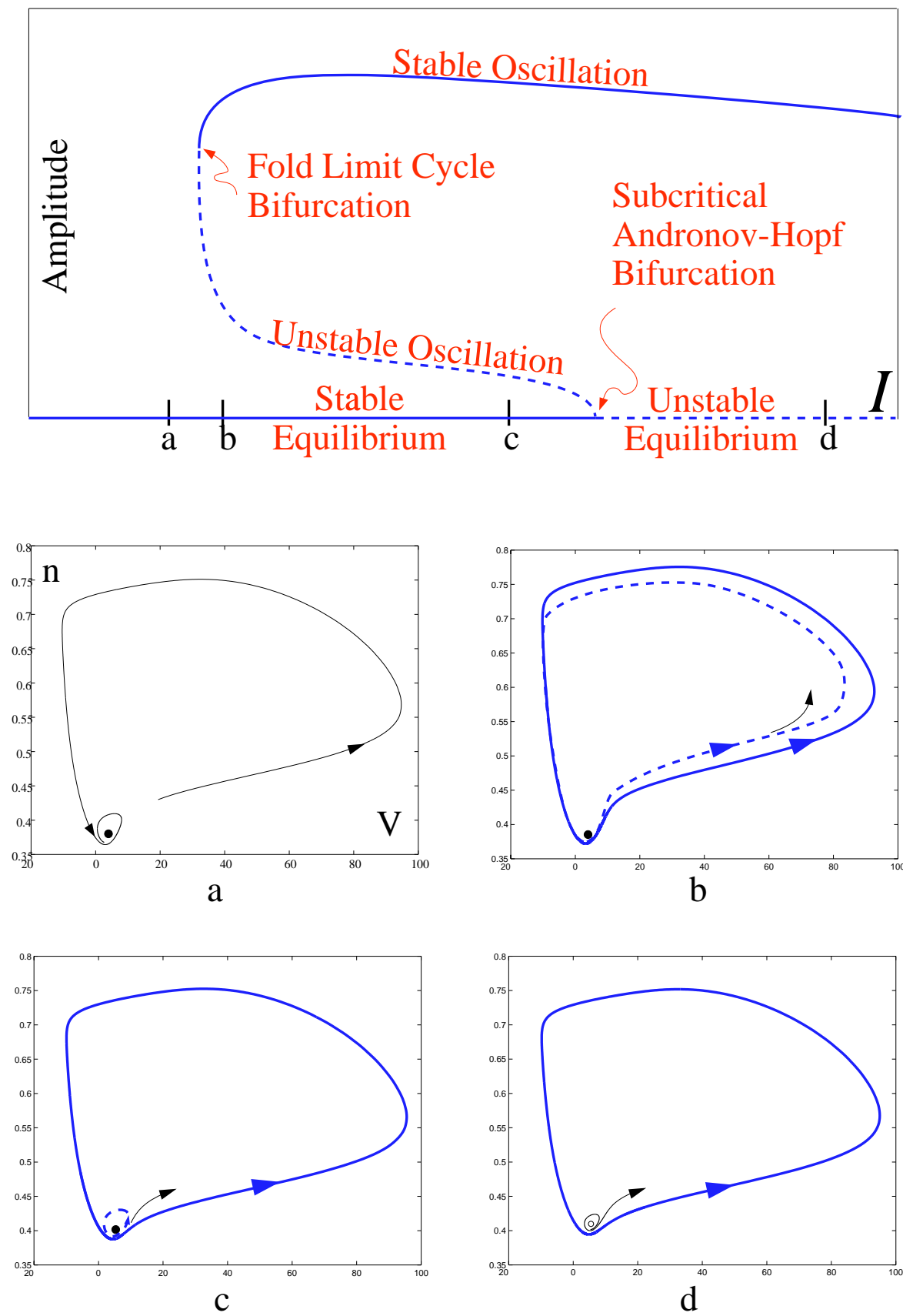

Fig. 16. Typical bifurcation structure in Hodgkin-Huxley model.

\subsubsection{Quasithreshold phenomenon}

The absence of a well-defined threshold manifold in the Hodgkin-Huxley model was discussed in detail by FitzHugh [1955], who made the following observation: The distance between the solutions producing small PSP and large spike in Fig. 17 can be as small as $0.1 \mu \mathrm{V}$, which is smaller than the noisy fluctuations of the membrane potential. Thus, to observe an intermediate-amplitude spike in the Hodgkin-Huxley model one needs to specify the initial condition with the accuracy beyond the limits of uncertainty which appear when the physical interpretation of the model is considered. As a result, the model exhibits all-or-none behavior almost all the time for almost all initial conditions, and its threshold set looks almost like a manifold. FitzHugh [1955] referred to this as being a quasithreshold phenomenon. 


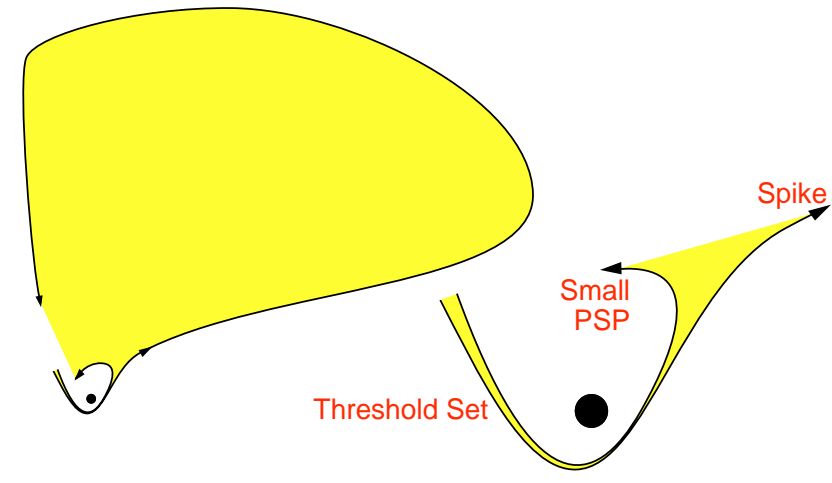

Fig. 17. Neural systems near Andronov-Hopf bifurcation may not have a well-defined threshold. Depicted are two "nearby" solutions of the Hodgkin-Huxley system from Fig. 16(a). One corresponds to a small amplitude postsynaptic potential (PSP), while the other evolves into an action potential.

\subsubsection{Response to doublets}

An excitable system at an Andronov-Hopf bifurcation possesses an important information processing capability: Its response to a pair (or a sequence) of stimuli depends on the timing between the stimuli relative to the period of the small amplitude damped oscillation at the equilibrium. We illustrate this in Fig. 18, which motivated the creation of a simple resonate-and-fire model [Izhikevich, 2001]. A pair of relatively strong pulsed perturbations (a doublet), may or may not evoke an action potential depending on its interspike interval. When the interval is near the period of the damped oscillations (or its multiple), then the effect of the perturbations can accumulate. Otherwise the perturbations may effectively cancel each other. Thus, the interspike interval in doublets or triplets plays an important role. This phenomenon is a part of the mechanism of FM (Frequency Modulated) interactions, and it is quite general in neural models [Hoppensteadt \& Izhikevich, 1998; Izhikevich, 1999a]. We discuss it in detail in Sec. 3.5.

\subsubsection{Selective communication and multiplexing}

The same doublet may or may not elicit response in a postsynaptic neuron depending on its eigenfrequency. This provides a powerful mechanism for selective communication between such neurons; see Fig. 19. In particular, such neurons can multiplex; i.e. send many messages via a single transmission line; see Fig. 20.

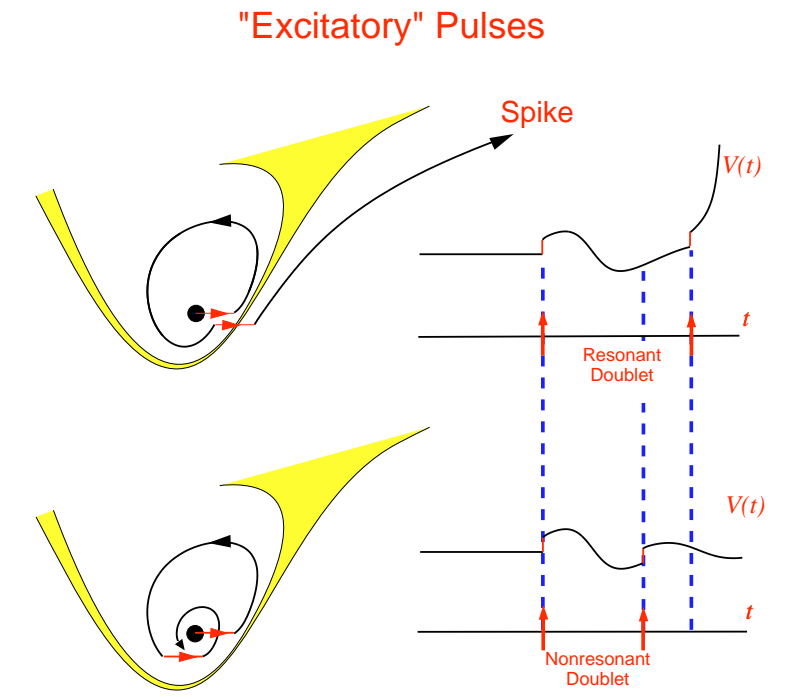

\section{"Inhibitory" Pulses}

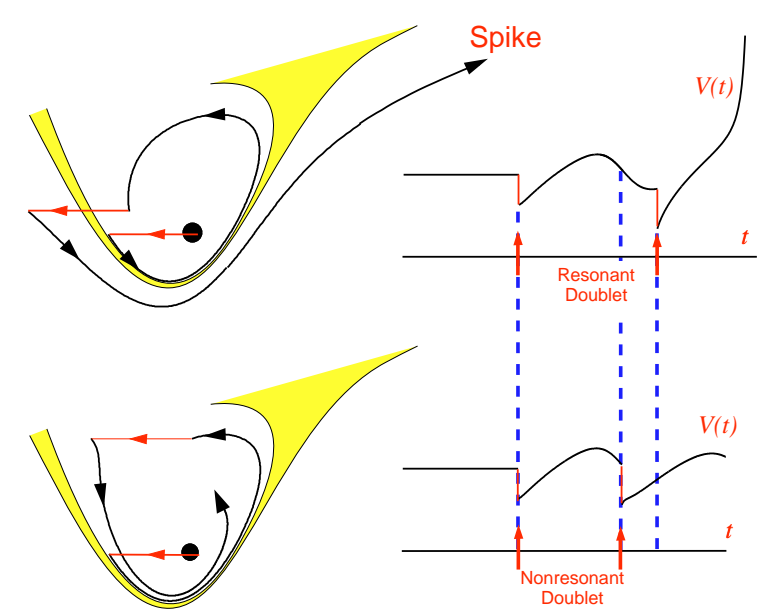

Fig. 18. Response of the Hodgkin-Huxley model to a doublet (i.e. a pair of pulses) depends on the length of the interspike interval. Notice that an "inhibitory" pulse can make the neuron fire.

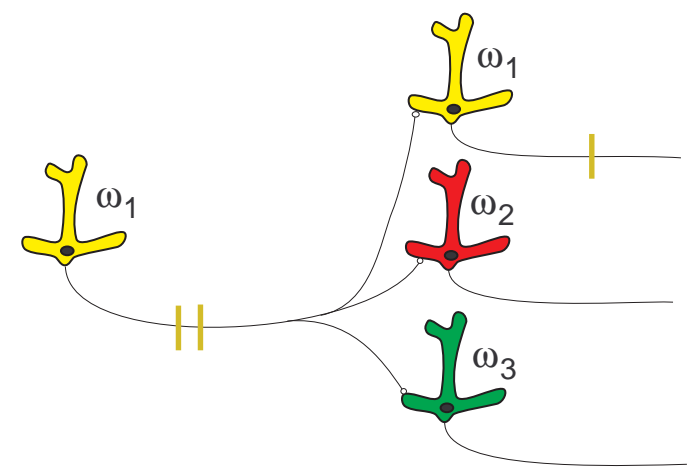

Fig. 19. Selective interaction: A doublet with the interspike frequency $\omega_{1}$ is resonant for another neuron having similar eigenfrequency and nonresonant for the others (from [Izhikevich, 2001]). 


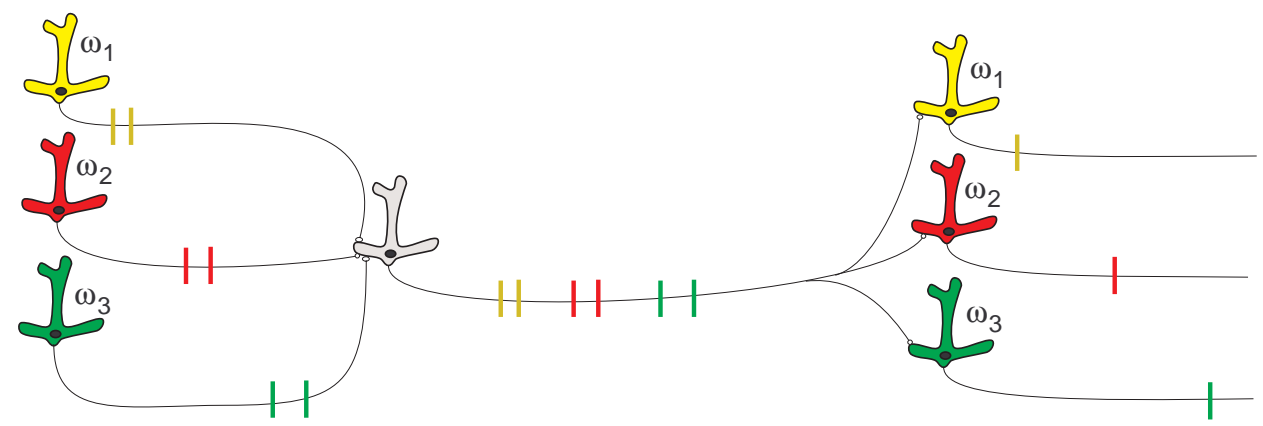

Fig. 20. Multiplexing of neural signals via doublets: Resonate-and-fire neurons having equal eigenfrequencies can interact selectively without any cross interference with other resonate-and-fire neurons (from [Izhikevich, 2001]).

\subsubsection{Weak stimulation}

Figure 18 is only an illustration, since presynaptic pulses do not elicit a step-like increase in membrane potential of postsynaptic Class 2 excitable neurons. They rather induce a weak and relatively slow forcing. Nevertheless, the FM mechanism persists, as we show next.

Let us disregard the global structure of the flow and consider its dynamics in a small neighborhood of the rest state. We are interested in a response of the system near the rest state to a weak external stimulation $\varepsilon I(t)$ that is due to incoming spikes.

It is well known that any system

$$
\dot{x}=f(x, \varepsilon), \quad x \in \mathbb{R}^{m}
$$

in an $\varepsilon$-neighborhood of the Andronov-Hopf bifurcation can be reduced to its topological normal form

$$
\dot{z}=(\varepsilon a+\mathrm{i} \omega) z \pm z|z|^{2}, \quad z \in \mathbb{C},
$$

by a continuous transformation. Applying the transformation to the weakly forced system

$$
\dot{x}=f(x, \varepsilon)+\varepsilon I(t)
$$

results in

$$
\dot{z}=\varepsilon J(t)+(\varepsilon a+\mathrm{i} \omega) z \pm z|z|^{2}
$$

plus higher-order terms, where $J(t) \in \mathbb{C}$ is a linear projection of $I(t) \in \mathbb{R}^{m}$. The change of variables

$$
z=\sqrt{\varepsilon} v e^{\mathrm{i} \omega t}, \quad v \in \mathbb{C},
$$

results in the equation

$$
\dot{v}=\sqrt{\varepsilon} J(t) e^{-\mathrm{i} \omega t}+\varepsilon\left(a v \pm v|v|^{2}\right) .
$$

We average this system and obtain the equation

$$
\dot{v}=\sqrt{\varepsilon} b+\varepsilon\left(a v \pm v|v|^{2}\right),
$$

where

$$
b=\lim _{T \rightarrow \infty} \frac{1}{T} \int_{0}^{T} J(t) e^{-\mathrm{i} \omega t} d t
$$

is the Fourier coefficient of $J(t)$ corresponding to frequency $\omega$. The key observation here is that $b$ can vanish even when $J(t) \neq 0$.

We assume that the equilibrium is stable, i.e. $a<0$. If $b=0$, then $v$, and hence $x$, stay near the equilibrium. In contrast, If $b \neq 0$, then $v$ grows like $\sqrt{\varepsilon} b t$ until $x=\mathcal{O}(\sqrt{\varepsilon} v)$ leaves a small neighborhood of the rest state, possibly getting outside of the unstable limit cycle in Fig. 16(c) and generating a spike.

\subsubsection{Class 2 excitable neurons are resonators}

We see that a system near an Andronov-Hopf bifurcation acts as a bandpass filter: It extracts the component of the external input $I(t)$ that corresponds to the "resonant" eigenfrequency $\omega$ and disregards the rest of the spectrum. Thus, in order to evoke a response, one should stimulate such a neuron at the resonant frequency. This behavior has been described in thalamic [Hutcheon et al., 1994; Puil et al., 1994] and cortical neurons [Jansen \& Karnup, 1994; Gutfreund et al., 1995; Hutcheon et al., 1996]. Llinas [1988, 1991] refers to such neurons as being resonators. In contrast to Class 1 excitable neurons, increasing the frequency of stimulation may delay or even terminate firing of a Class 2 excitable neuron [Izhikevich, 2001], since it may decrease the value of $|b|$ defined by (14). We return to this issue in Sec. 3.5 when we discuss FM interactions. 


\subsubsection{Post-inhibitory spike}

A salient neuro-computational feature of Class 2 excitable neurons is that they can fire in response to a weak inhibitory pulse (see Fig. 18), which is referred to as being a post-inhibitory spike. This makes the distinction between excitation and inhibition a bit confusing, since both can lead to an action potential. Such a confusion does not exist in Class 1 excitable neurons, because weak excitatory (inhibitory) pulses decrease (increase) the distance to the threshold manifold; see Fig. 14. Since the threshold set is always "wrapped" around the rest state of Class 2 excitable neuron, any perturbation would eventually move the solution closer to the set, thereby facilitating the neuron's response to other pulses having appropriate timing.

\subsection{Saddle-node separatrix-loop bifurcation}

Now consider the case of fold (off limit cycle) bifurcation as in Fig. 17. If the limit cycle is sufficiently far away from the saddle-node point, then its frequency is generically nonzero, and hence such a bistable system has Class 2 excitability.

Quite often however the limit cycle is near the saddle-node point, as in Fig. 21. This usually happens when the system is near a codimension 2 bifurcation called saddle-node separatrix-loop bifurcation [Levi et al., 1978; Schecter, 1987; Hoppensteadt, 1997], whose complete unfolding is depicted in Fig. 22. The saddle-node separatrix-loop bifurcation is typical in two-dimensional systems having nullclines intersected as in Fig. 23. Such systems include Morris-Lecar, Chay-Cook, and WilsonCowan models.

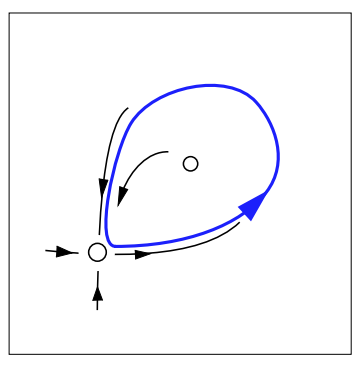

Fold (off Limit Cycle) Bifurcation

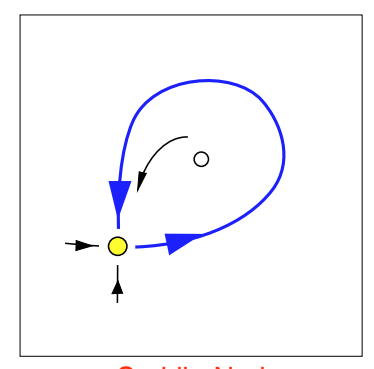

Saddle-Node Separatrix-Loop Bifurcation
Fig. 21. Fold bifurcation can be near a limit cycle if the system is near a saddle-node separatrix loop bifurcation.

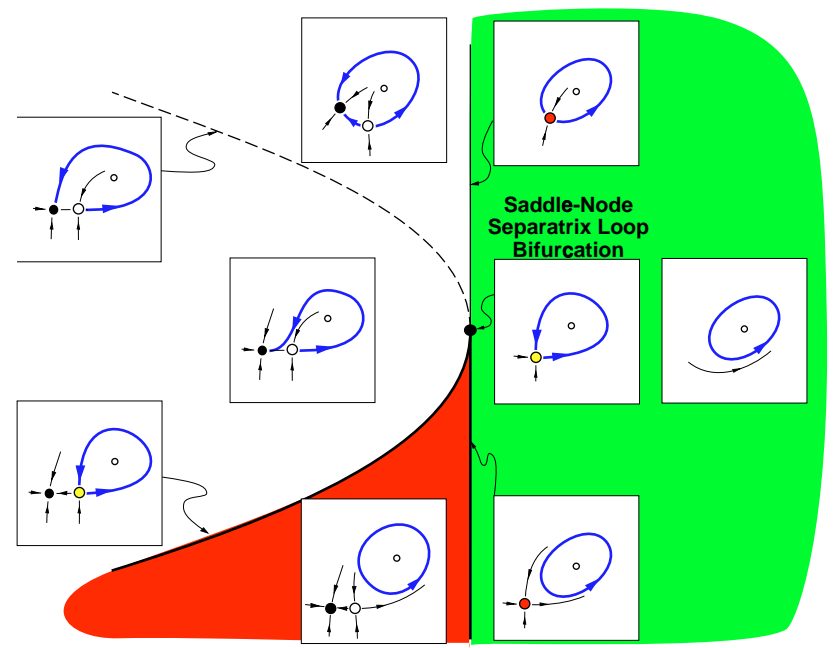

Fig. 22. Unfolding of a codimension 2 saddle-node separatrix loop bifurcation (from [Hoppensteadt \& Izhikevich, 1997]).
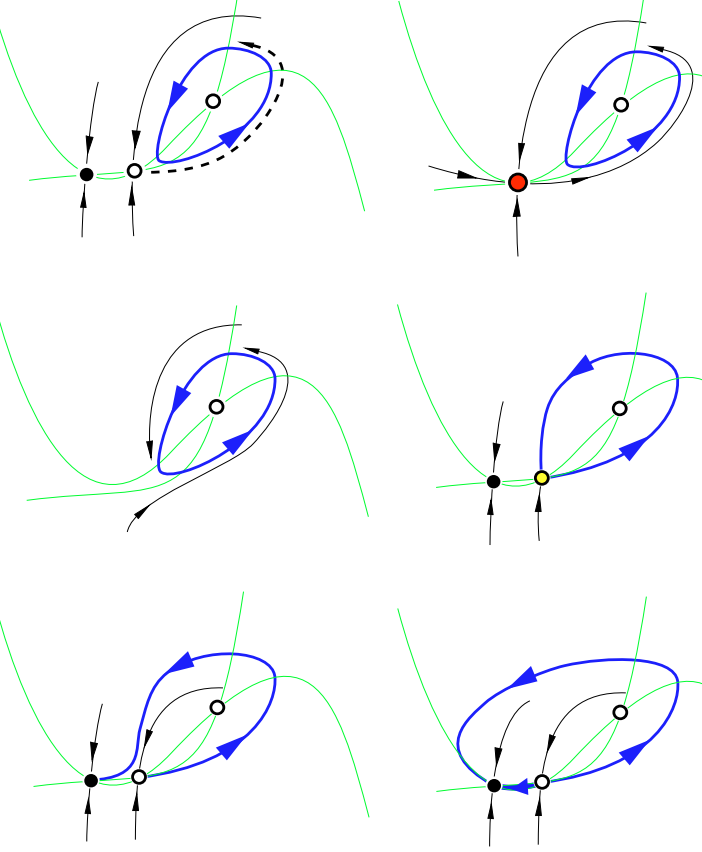

Fig. 23. Phase portraits from Fig. 22 are typical in twodimensional systems, such as Wilson-Cowan or Morris-Lecar models (modified from [Hoppensteadt \& Izhikevich, 1997]).

As we will see in Sec. 4 below, this bifurcation plays an important role in at least four types of bursting including the "fold/homoclinic" bursting, which is also known as "square-wave" bursting.

\subsubsection{Canonical model}

It follows from the normally hyperbolic compact invariant manifold theory [Fenichel, 1971; 


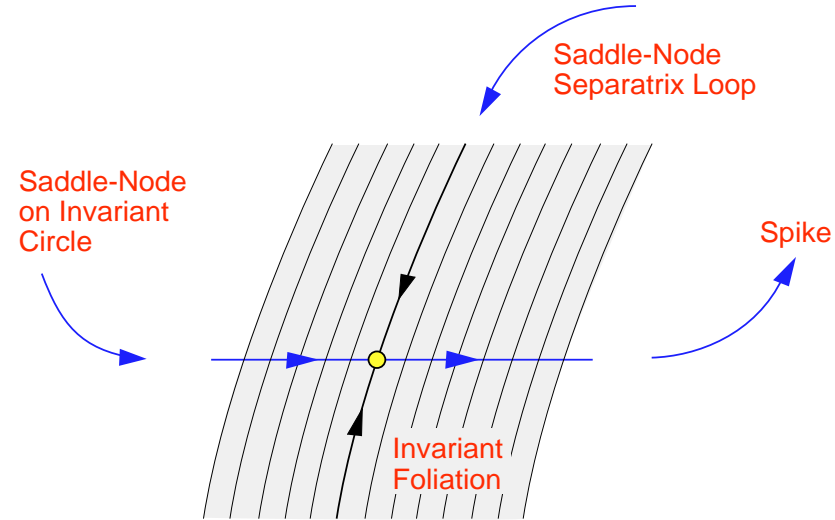

Fig. 24. A small neighborhood of the saddle-node point can be invariantly foliated by stable submanifolds.

Hoppensteadt \& Izhikevich, 1997, Chap. 4] that a small neighborhood of the saddle-node point can be invariantly foliated by stable submanifolds, which are frequently referred to as being isochrons in the context of oscillatory systems; see Fig. 24. Any two distinct solutions starting on the same submanifold will eventually approach each other and have identical asymptotic behavior. This fact was used in the proof of the Ermentrout-Kopell theorem to reduce the dimension of a system.

Now consider a system near a saddle-node separatrix loop bifurcation. All solutions in some small neighborhood of the saddle-node point (shaded area in Fig. 24) approach exponentially the center manifold. A solution on the center manifold slowly diverges from the equilibrium, makes an excursion (spike) and returns to the neighborhood (shaded area). But it enters the neighborhood along one of the stable submanifolds. The spike occurs when the canonical variable $\vartheta$ in (2) crosses a tiny neighborhood of $\pi$, but instead of being reset to $-\pi$, the variable $\vartheta$ acquires some new value $a \in \mathbb{S}^{1}$ that is determined by the location of the stable submanifold that was hit by the separatrix loop; see Fig. 25 .

Thus, one can prove under certain natural conditions that the canonical model for the saddlenode separatrix-loop bifurcation has the form (2) with the exception that

$$
\vartheta(t) \leftarrow a \quad \text { when } \vartheta(t)=\pi .
$$

The parameter $r$ in the canonical model is local in the sense that it depends on some partial derivatives near the equilibrium [Hoppensteadt \& Izhikevich, 1997, Chap. 8]. In contrast, the parameter $a$ is global since it depends on Melnikov's integral along the unperturbed separatrix trajec-
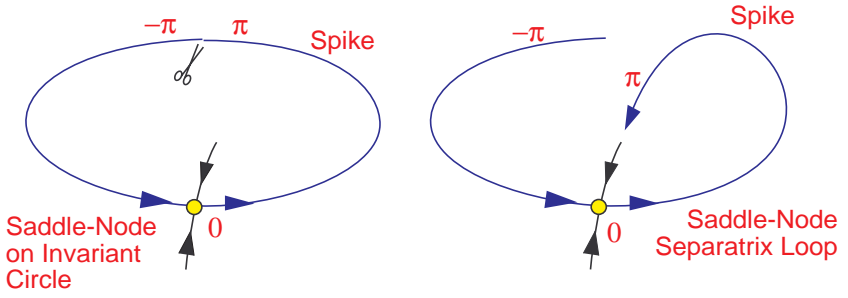

Fig. 25. The saddle-node separatrix loop bifurcation can be treated the same way as the saddle-node on invariant circle bifurcation with the exception that the variable $\vartheta$ in (2) is reset to some value $a$ after crossing $\pi$.

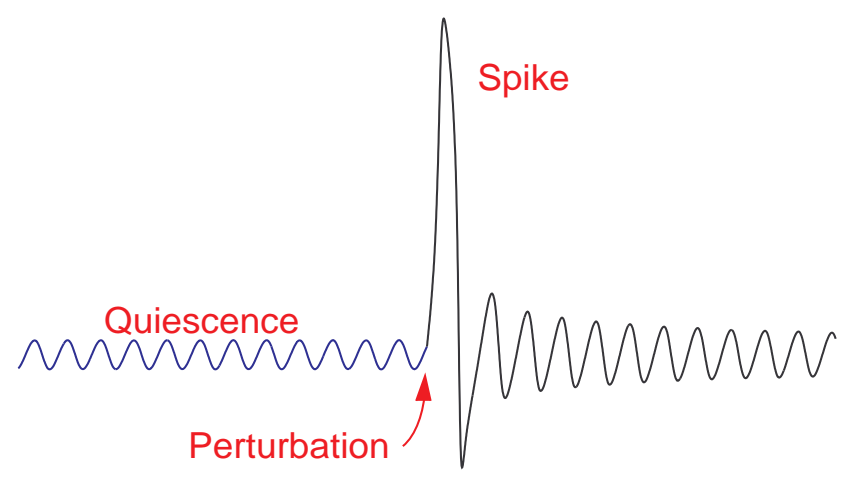

Fig. 26. An example of a small amplitude subthreshold oscillation (blue) corresponding to the quiescent state.

tory. The canonical model exhibits a fold bifurcation when $r=0$, and a saddle homoclinic orbit bifurcation when $r<0$ and $\cos a=(1+r) /(1-r)$. Its bifurcation diagram is similar to the one depicted in Fig. 22, where the $a$-axis is pointed downward.

\subsection{Fast subthreshold oscillations}

So far we have considered neuron dynamics at an equilibrium corresponding to the rest (quiescent) state. Next suppose that the membrane potential has a fast stable small amplitude "subthreshold" oscillation corresponding to the quiescent state, as we illustrate in Fig. 26. Such neurons have been recorded, e.g. in layer 4 of the guinea pig frontal cortex [Llinas et al., 1991]. Fast subthreshold oscillations can be seen in conductance-based models [Shorten \& Wall, 2000; Wang, 1993].

The simplest and possibly least interesting case is when the neuron's activity is always oscillatory in a certain parameter range with an amplitude proportional to the external input, as in Fig. 27. In this case the distinction between the sub- and superthreshold oscillations may be made by the synaptic release mechanism; that is, the amplitude of 


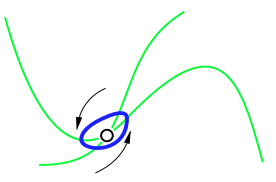

No External Input

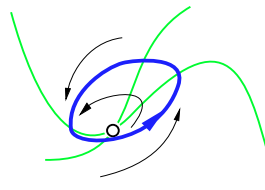

Small External Input

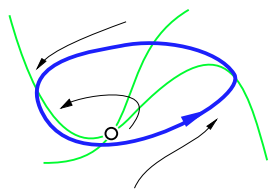

Large External Input

Fig. 27. The amplitude of oscillation may depend on the external input.

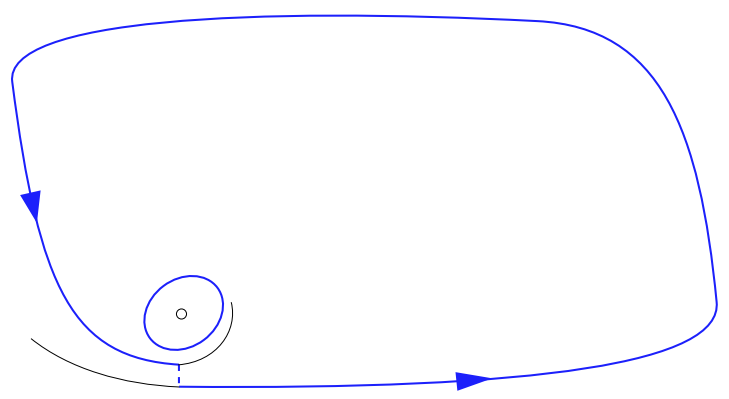

Fig. 28. Existence of a large amplitude periodic pseudoorbit near small amplitude "subthreshold" limit cycle makes the dynamics excitable.

oscillation is said to be superthreshold if it is large enough to trigger the release of a neurotransmitter from the presynaptic endings.

Notice that small amplitude subthreshold oscillations do not preclude the system from being excitable, since it can still have a large amplitude periodic pseudo-orbit; see Fig. 28.

\subsubsection{Possible bifurcations}

In a more interesting case the small amplitude limit cycle may bifurcate so that some large amplitude limit cycle corresponding to a periodic spiking becomes globally stable.

Obviously, the saddle-node on invariant circle and the supercritical Andronov-Hopf bifurcation should be dismissed as possible bifurcations, since they result in a stable equilibrium and not in a large amplitude limit cycle. Similarly, the supercritical flip (period doubling) and supercritical NeimarkSacker bifurcations [Kuznetsov, 1995] should be discarded since the newborn attractors lie in a small neighborhood of the old ones, which is often referred to as a soft loss of stability.

We consider next bifurcations that result in sharp loss of stability. These are the fold limit cycle and saddle homoclinic orbit bifurcations in the planar case (see Fig. 29), and the saddle-focus homoclinic orbit, subcritical flip, subcritical NeimarkSacker, fold limit cycle on homoclinic torus, and
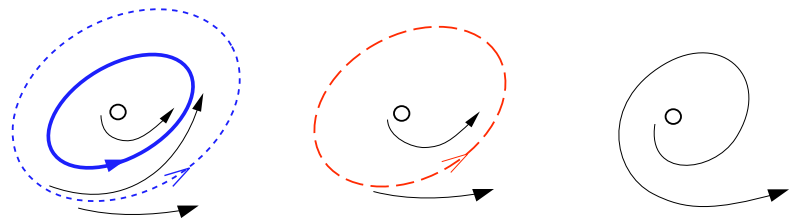

Fold Limit Cycle Bifurcation
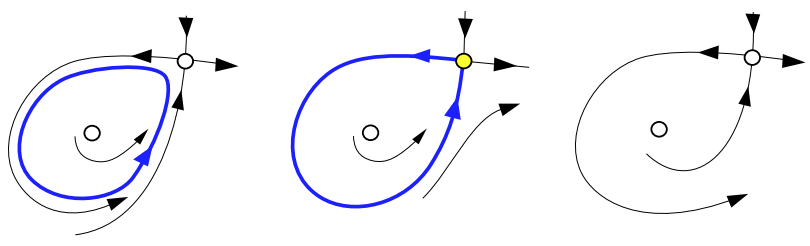

Saddle Homoclinic Orbit Bifurcation

Fig. 29. Codimension 1 bifurcations of a stable limit cycle in planar systems that result in sharp loss of stability (see also Fig. 30). Fold limit cycle: Stable limit cycle is approached by an unstable one, they coalesce, and then disappear. Saddle homoclinic orbit: A limit cycle grows into a saddle. Unstable manifold of the saddle makes a loop and returns via the stable manifold (separatrix).

the "blue-sky" bifurcations in the three-dimensional case; see Fig. 30. The only bifurcation left is the focus-focus homoclinic orbit, which we do not illustrate since it occurs in systems of dimension 4 and up; see [Kuznetsov, 1995].

The planar bifurcations are ubiquitous in twodimensional systems having nullclines intersected as in Fig. 31. Notice the shape of the slow nullcline for the saddle homoclinic orbit bifurcation. One can easily modify existing models, such as the van der Pol or FitzHugh-Nagumo oscillators, to get such dynamics. For example, to make Fig. 34 we used

$$
\begin{aligned}
\dot{v} & =v-v^{3} / 3-w \\
\dot{w} & =\varepsilon(a+v-S(w))
\end{aligned}
$$

where

$$
S(w)=\frac{b}{1+e^{(c-w) / d}},
$$

is an $S$-shaped function. Such a system often exhibits the big saddle homoclinic orbit bifurcation depicted in Fig. 32.

There is a drastic change in the behavior of the small limit cycle when the system approaches the bifurcation state. Its frequency is nonzero for the fold limit cycle, flip, Neimark-Sacker, blue-sky, and fold limit cycle on homoclinic torus bifurcations, and zero for the homoclinic orbit bifurcations since the cycle becomes a homoclinic trajectory to an equilibrium. This gives a criterion to distinguish the bifurcations experimentally. Both cases, 


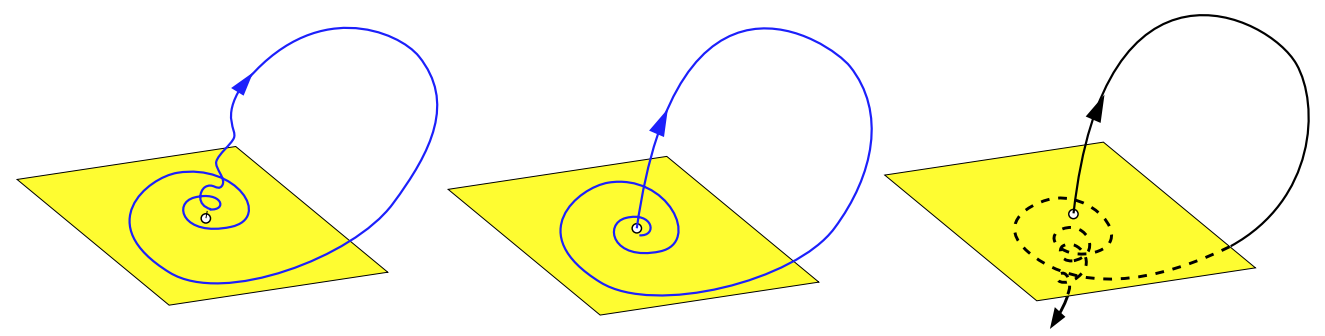

Saddle-Focus Homoclinic Orbit Bifurcation
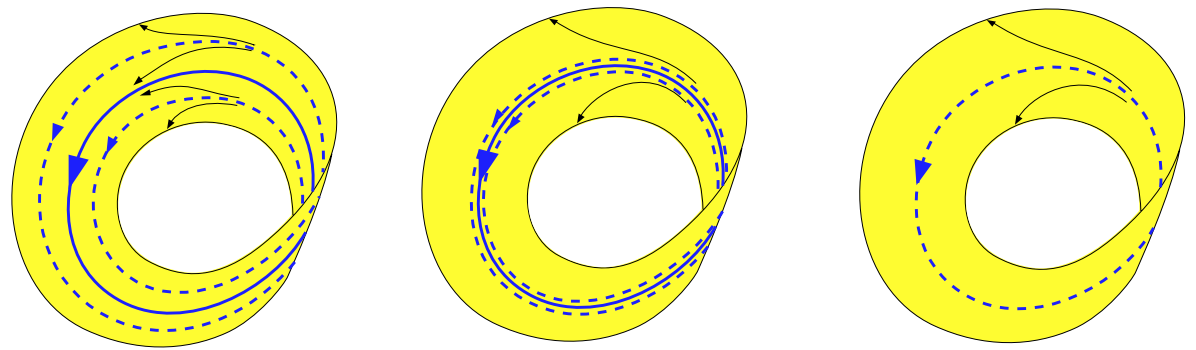

Subcritical Flip Bifurcation
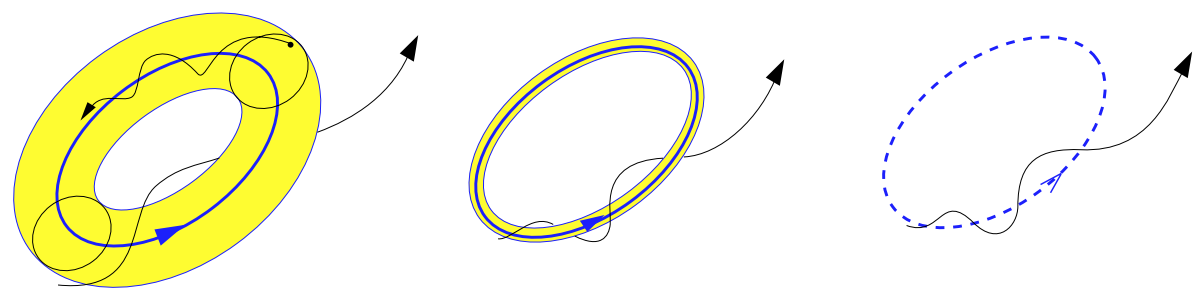

Subcritical Neimark-Sacker Bifurcation
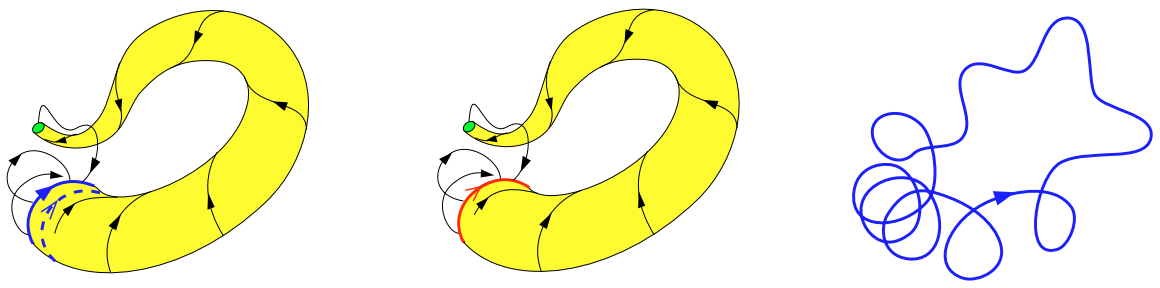

Blue-Sky Catastrophe
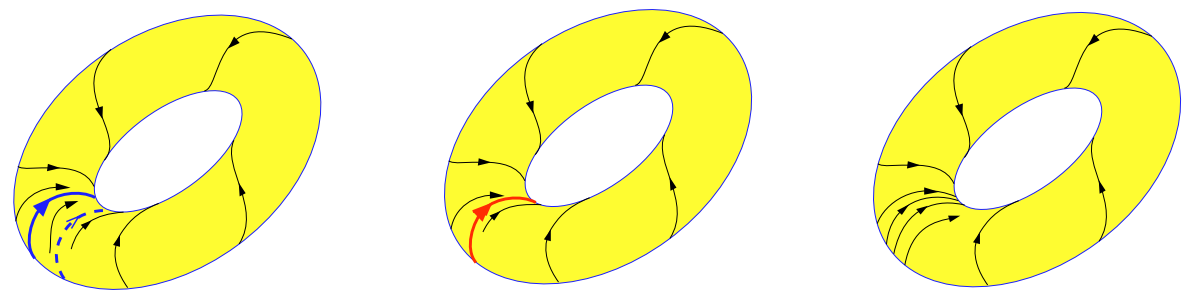

Fold Limit Cycle on Homoclinic Torus Bifurcation

Fig. 30. Codimension 1 bifurcations of a stable limit cycle in three-dimensional systems that result in sharp loss of stability (see also Fig. 29). Saddle-focus homoclinic orbit: A limit cycle grows into a saddle-focus and becomes a homoclinic orbit. Subcritical flip: The stable limit cycle is approached by an unstable limit cycle having twice the period, they coalesce, and the former loses stability. Subcritical Neimark-Sacker: An unstable invariant torus shrinks down to a stable limit cycle. Fold limit cycle on homoclinic torus: An unstable manifold of a nonhyperbolic limit cycle returns to the cycle forming a homoclinic torus. Blue-sky catastrophe: An unstable manifold of a nonhyperbolic limit cycle becomes a tube and returns to the cycle forming "French horn"; see [Kuznetsov, 1995; Il'iashenko \& Li, 1999] for detailed definitions. 

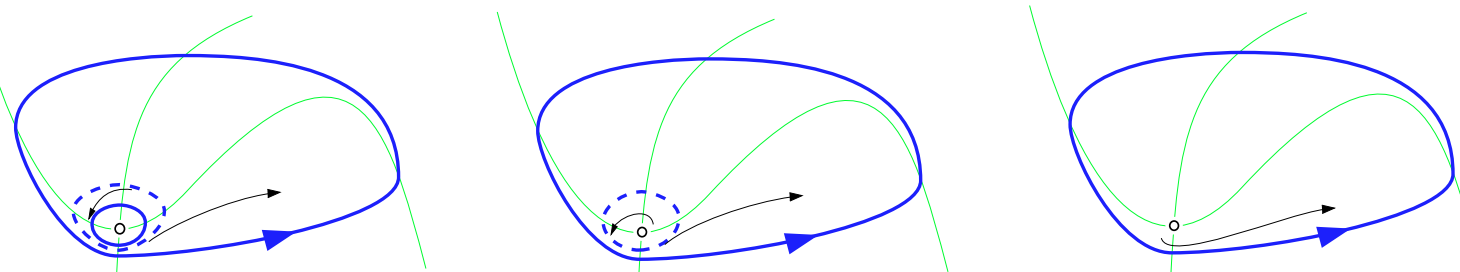

Fold Limit Cycle Bifurcation
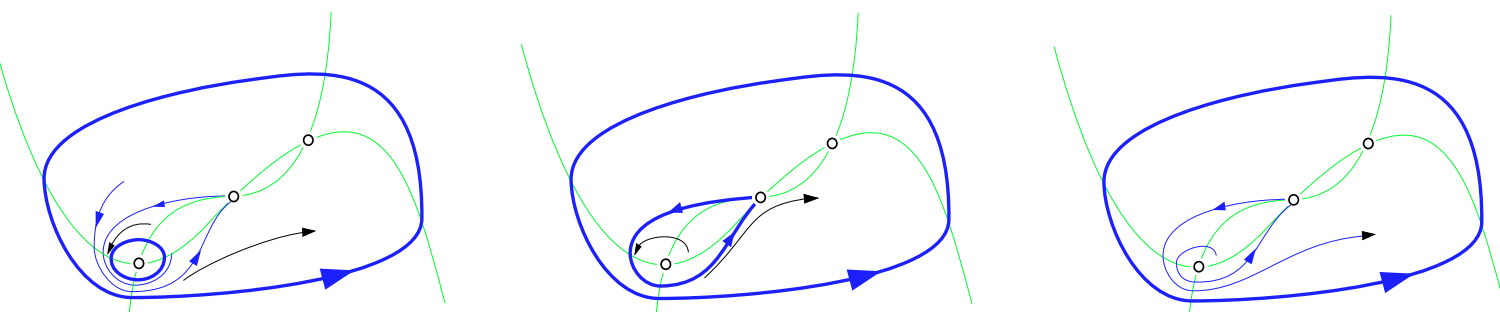

Saddle Homoclinic Orbit Bifurcation

Fig. 31. Typical intersections of nullclines for fold limit cycle and saddle homoclinic orbit bifurcations.

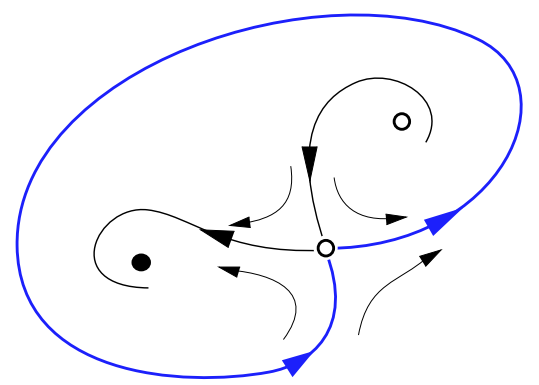

Fig. 32. Big saddle homoclinic orbit (from [Hoppensteadt \& Izhikevich, 1997]).

i.e. zero and nonzero frequency, were recorded in vitro by Llinas et al. [1991].

Below we discuss neuro-computational properties of neurons having subthreshold oscillation. Our results are summarized in the lower part of Table 1.

\subsubsection{Class of excitability}

Recall that the class of excitability is determined not by the frequency of the subthreshold oscillation, but by the frequency of the emerging spiking; that is, by the frequency of the large amplitude limit cycle, such as the one in Fig. 31. The figure suggests that the frequency may be nonzero in those two planar cases, thus resulting in Class 2 excitability. Similarly, the saddle-focus homoclinic orbit, subcritical flip, and subcritical Neimark-Sacker bifurcations result in Class 2 excitability unless the system is near certain codimension 2 bifurcations.
In contrast, the blue-sky catastrophe and the fold limit cycle on homoclinic torus bifurcation result in Class 1 excitability. Indeed, in both cases the solution spends most of its time rotating around the recently disappeared small amplitude fold limit cycle thereby producing long-period spiking.

\subsubsection{Threshold and excitability}

Figure 31 illustrates bistable dynamics of planar systems having a fold limit cycle bifurcation. There is a well-defined threshold manifold: The intermediate, unstable limit cycle.

In contrast, when the fold limit cycle has a homoclinic structure; e.g. a homoclinic orbit or a torus, then the system has a periodic pseudo-orbit (see Fig. 33), and hence is only excitable. Local behavior near such a fold cycle is similar to that near a saddle-node on invariant circle where stable, unstable, and fold limit cycles correspond to node, saddle, and saddle-node equilibria, respectively. The system has a well-defined threshold manifold the union of an unstable limit cycle and its stable submanifold (yellow region in Fig. 35). Such an oscillatory system acts as an integrator.

The saddle, saddle-focus, and focus-focus homoclinic orbit bifurcations have qualitatively similar neuro-computational properties: They have well-defined thresholds; namely, the saddle's stable manifold. The systems may be bistable (Fig. 31) or 


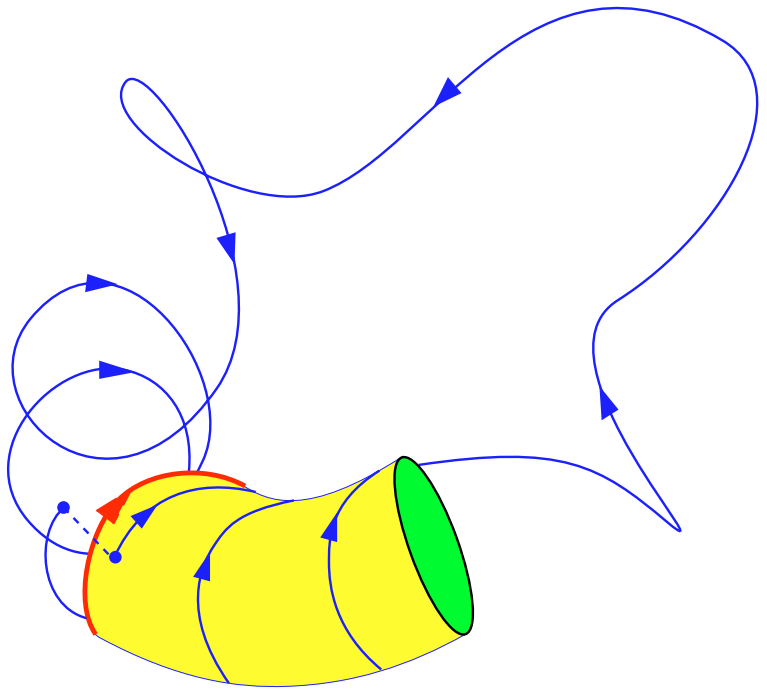

Fig. 33. An existence of a large amplitude periodic pseudoorbit near small amplitude "subthreshold" fold limit cycle makes the dynamics excitable.

only excitable (Fig. 34) depending on the existence of an enclosing limit cycle.

Stable limit cycles near subcritical flip and Neimark-Sacker bifurcations also have well-defined threshold manifolds; namely, the stable submanifold of unstable double-period cycle and the unstable torus, respectively.

\subsubsection{Nonlinear resonators}

Neural systems having subthreshold oscillations may have an interesting property: Their response to a brief relatively strong stimulus depends on the timing of the stimulus relative to the phase of the oscillation. We illustrate this issue in Fig. 34 using (15) near the saddle homoclinic orbit bifurcation. It is easy to see that the response depends on where the solution is on the limit cycle at the time of the perturbation.

There is a similarity between the mechanisms depicted in Figs. 18 and 34. Both use the fact that a neuron may have its own subthreshold temporal dynamics that affect its response to a perturbation. The only difference is that the former cannot oscillate by itself; therefore, it needs a first spike to evoke a damped subthreshold oscillation whose phase would augment the response to the second spike.
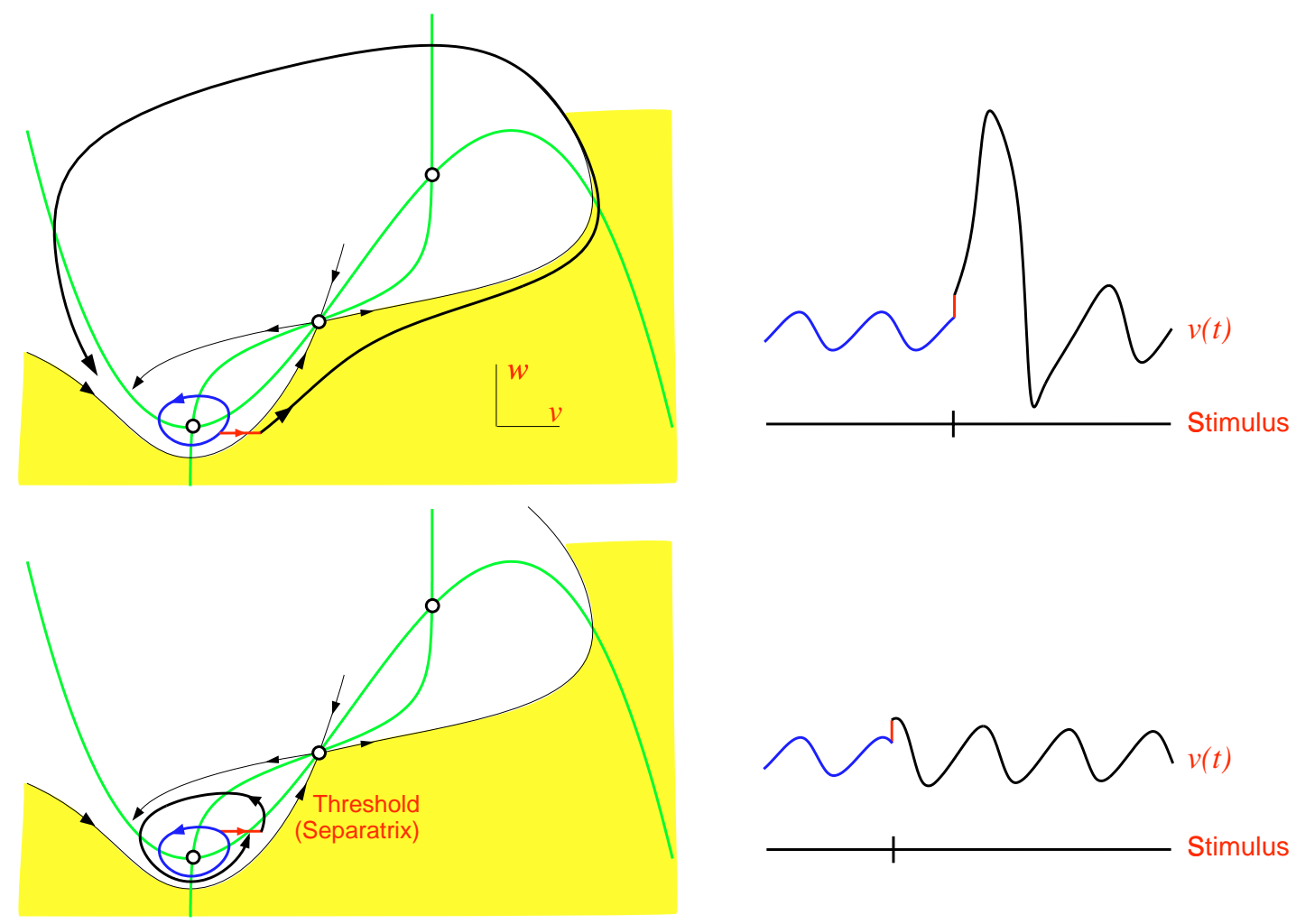

Fig. 34. Response of a system having a small amplitude oscillation depends on the timing of the incoming spike relative to the phase of the subthreshold oscillation. 


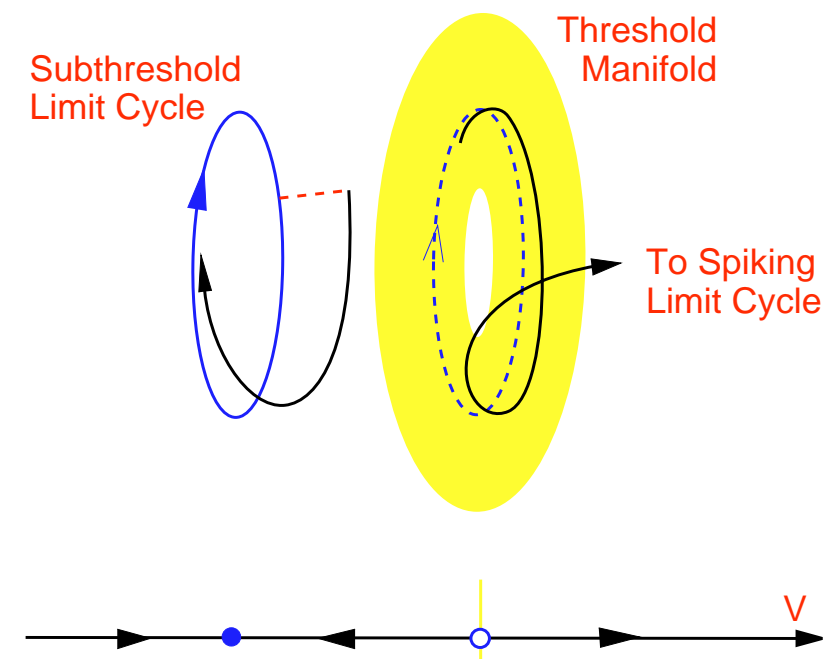

Fig. 35. A system near fold limit cycle bifurcation can still act as integrator: The effect of perturbations does not depend on the subthreshold oscillation phase. Moreover, increasing the subthreshold oscillation amplitude does not decrease the distance to the threshold manifold.

We could have also used any other bifurcations (except blue-sky and homoclinic torus) to illustrate the mechanism depicted in Fig. 34. A distinguished feature of the saddle homoclinic orbit bifurcation is that there is an open region above the cycle in Fig. 34 such that if perturbations are directed toward this region, then they would never elicit a spike regardless of their timing and strength.

Finally, notice that the existence of subthreshold oscillation does not necessarily imply that the system is a resonator, especially when its dimension is greater than 2. For example, the system near fold limit cycle bifurcation depicted in Fig. 35 acts as an integrator. Although appropriately timed perturbations can change the amplitude of oscillation, but such a change does not decrease significantly the distance to the threshold manifold, and hence does not facilitate the spike, see also Fig. 122.

\section{Periodic Spiking}

So far we have described mechanisms of transition from a quiescent state to repetitive firing; that is, from an equilibrium or a small amplitude limit cycle to a large amplitude limit cycle attractor. How does the large attractor arise?

Much insight on possible mechanisms for the appearance of the large attractor can be gained when we consider possible mechanisms of transition
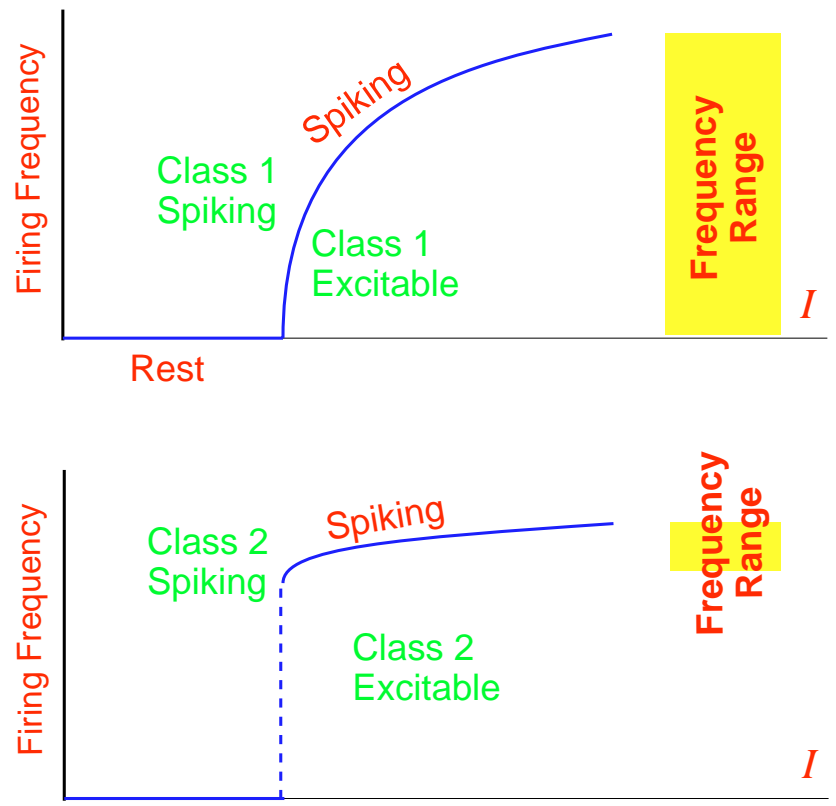

Rest

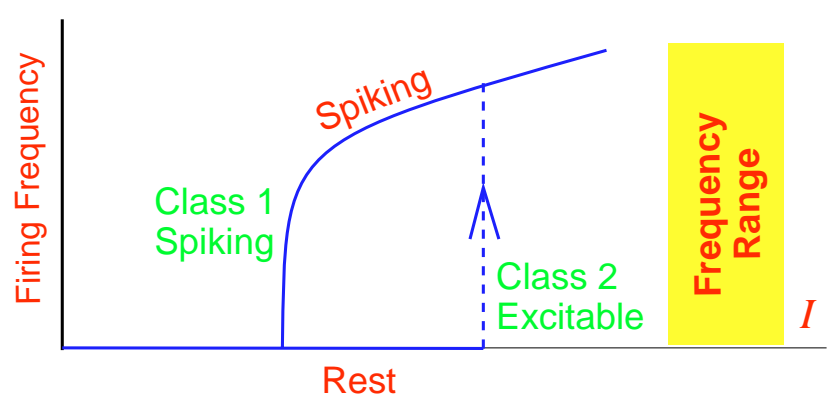

Fig. 36. Class 1 (2) excitable systems exhibit zero (nonzero) emerging spiking. Class 1 (2) spiking systems exhibit zero (nonzero) terminating spiking.

from repetitive spiking to a rest state. Following Hodgkin's experiment [1948] we suggest the following classification of repetitive spiking based on the frequency as oscillations terminate; see Fig. 36.

- Class 1 Spiking Systems exhibit terminating oscillations having arbitrary low frequency.

- Class 2 Spiking Systems exhibit oscillations that terminate with a nonzero frequency.

We stress that studying terminating oscillations provides a clue about how the attractor corresponding to repetitive spiking appears and disappears. It usually does not provide any information on how the spiking activity appears. The latter issue is related to neural excitability, as discussed in the previous section.

It is easy to see that a saddle-node on invariant circle bifurcation results in a system that is 


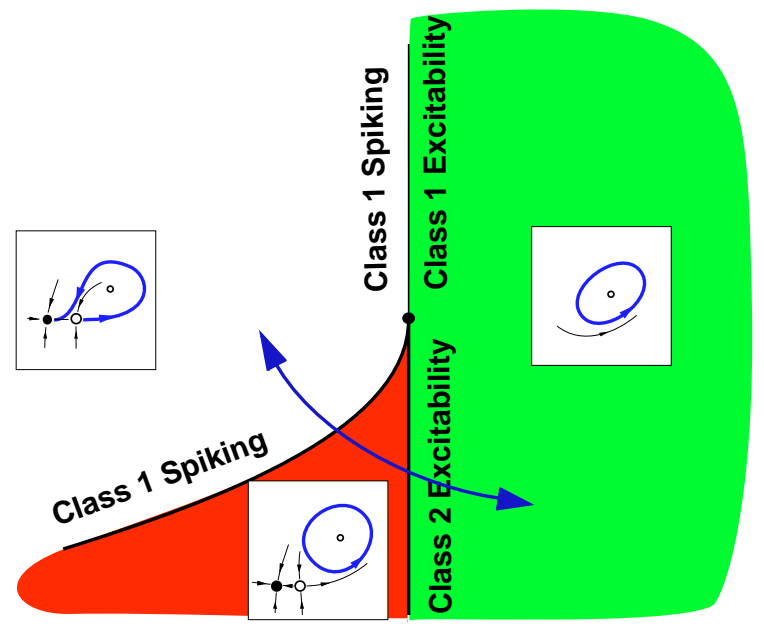

Fig. 37. If the rest state disappears via fold (off limit cycle) bifurcation and the limit cycle disappears via saddle homoclinic orbit bifurcation (see Fig. 22 for reference), then the system is Class 2 excitable but Class 1 spiking.

simultaneously Class 1 excitable and Class 1 spiking. Similarly, the supercritical Andronov-Hopf bifurcation results in a Class 2 excitable and Class 2 spiking system. In both cases the transitions from rest to repetitive firing and back occur via the same bifurcation.

In general, the bifurcation of the rest state may not be the same as the bifurcation of the limit cycle. In this case a stable rest state and a stable limit cycle coexist making the dynamics bistable, and the class of excitability may not be the same as the class of spiking. We will present several such examples when we discuss bursting.

An example of Class 2 excitable but Class 1 spiking system is the fold (off limit cycle) bifurcation. If the system is sufficiently away from the codimension 2 saddle-node separatrix-loop bifurcation (see Figs. 22 and 37), then the frequency of the limit cycle is not zero at the moment the rest state disappears. This results in Class 2 excitability. The limit cycle can disappear via a saddle-node on invariant circle or saddle homoclinic orbit bifurcation. Both bifurcations are homoclinic, and hence they result in Class 1 spiking.

Possible Bifurcations. When a repetitive spiking activity shuts down, the limit cycle attractor either loses its stability or disappears. If it loses stability via a soft bifurcation, such as the supercritical flip (period doubling) or supercritical Neimark-Sacker, the new attractor lies in a small neighborhood of the old one, so that the system continues to fire but with a different firing pattern. To shut down the firing, the loss of stability must be sharp; e.g. via a subcritical flip or subcritical Neimark-Sacker bifurcations; see Fig. 30.

The large amplitude limit cycle can also disappear, for example, via saddle homoclinic orbit or fold limit cycle bifurcation (Fig. 29), saddle-node on invariant circle or supercritical Andronov-Hopf bifurcation (Fig. 7), or those from Fig. 30.

We will see that

- Class 1 spiking occurs via saddle-node on invariant circle, saddle, saddle-focus, or focus-focus homoclinic orbit, blue-sky, or fold limit cycle on homoclinic torus bifurcations.

- Class 2 spiking occurs via supercritical Andronov-Hopf, fold limit cycle, subcritical flip, or subcritical Neimark-Sacker bifurcations.

Bifurcations and Current $I$. The most obvious way to study bifurcations in neuron dynamics is to use the same electrode that measures the membrane voltage to induce the current $I$, which we treat as a bifurcation parameter. There could be many interpretations of the physiological meaning of $I$. For example, we can interpret $I$ as a synaptic current

$$
I=g_{\mathrm{syn}}\left(E_{\mathrm{syn}}-V\right)
$$

or as the current that is due to the difference of voltages in soma and dendrites

$$
I=g\left(V_{\mathrm{dendr}}-V\right)
$$

etc. Thus, the "actual" bifurcation parameters in the spiking mechanism are $g_{\text {syn }}, V_{\text {dendr }}$, etc., but not $I$. Therefore, instead of changing $I$, we should slowly change, e.g. $g_{\text {syn }}$, measure the voltage, and inject $I$ calculated according to (16), which involves the dynamic clamp techniques [Sharp et al., 1993; Hutcheon et al., 1996]. Both procedures are equivalent when the membrane potential is at rest, but they may provide quite different bifurcation pictures when the potential oscillates. Thus, one should use (16) or its equivalent to study bifurcations of spiking limit cycles.

Another potential problem is that the rate of change of a bifurcation parameter should be slow enough so that it is treated as a constant by the fast spiking subsystem. Yet it should not be too slow, or else slow physiological processes start to interfere and may significantly distort the bifurcations picture. 


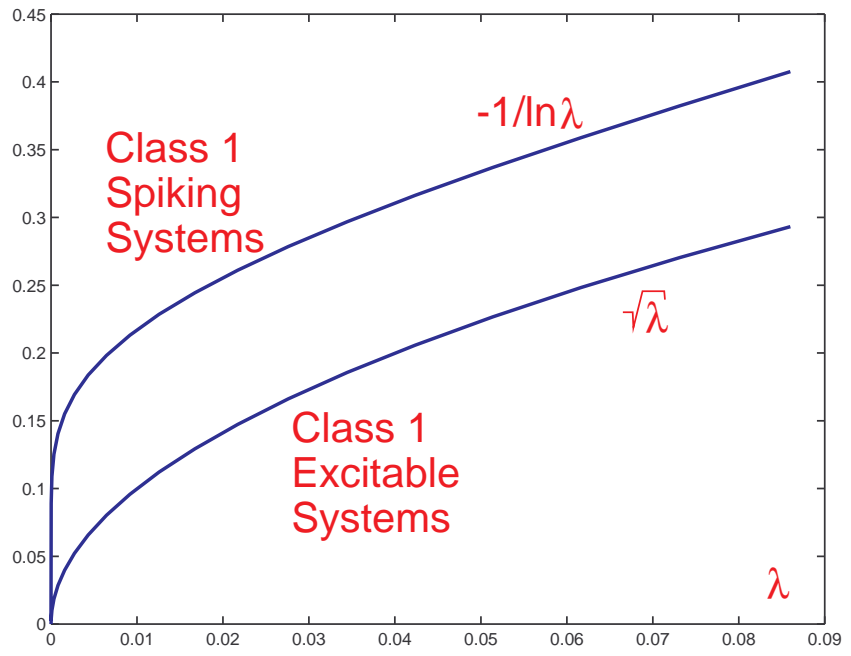

Fig. 38. The function $-1 / \ln \lambda$ may not seem to vanish as $\lambda \rightarrow 0$.

\subsection{Class 1 spiking systems}

Suppose the limit cycle disappears via one of the following bifurcations:

- Saddle-node on invariant circle bifurcation, or blue-sky catastrophe.

- Saddle, saddle-focus, or focus-focus homoclinic orbit bifurcations.
In all these cases the cycle becomes homoclinic to an equilibrium, hence its period goes to infinity, and the frequency to zero. All these bifurcations result in Class 1 spiking, but the frequency of terminating oscillations have different asymptotic behavior: The former group's frequency decays as $\mathcal{O}(\sqrt{\lambda})$, where $\lambda$ measures the distance to the bifurcation (see Sec. 2.1), while the latter exhibits $\mathcal{O}(1 /|\ln \lambda|)$. We plot these functions in Fig. 38 to illustrate a possible pitfall: The latter may not seem to vanish as $\lambda \rightarrow 0$, incorrectly suggesting a frequency curve for Class 2 spiking system. Thus, extra caution should be used when measuring the functions experimentally.

\subsubsection{Threshold and bistability}

An important difference between the bifurcations leading to Class 1 spiking is that there may be no coexistence of attractors at the saddle-node on invariant circle and blue-sky bifurcations, while there is at the saddle homoclinic orbit bifurcations. Thus, the former may not result in bistable dynamics. A system is either excitable or oscillatory, and no pulse can shut down the oscillation. In contrast, the latter always result in bistable systems having a
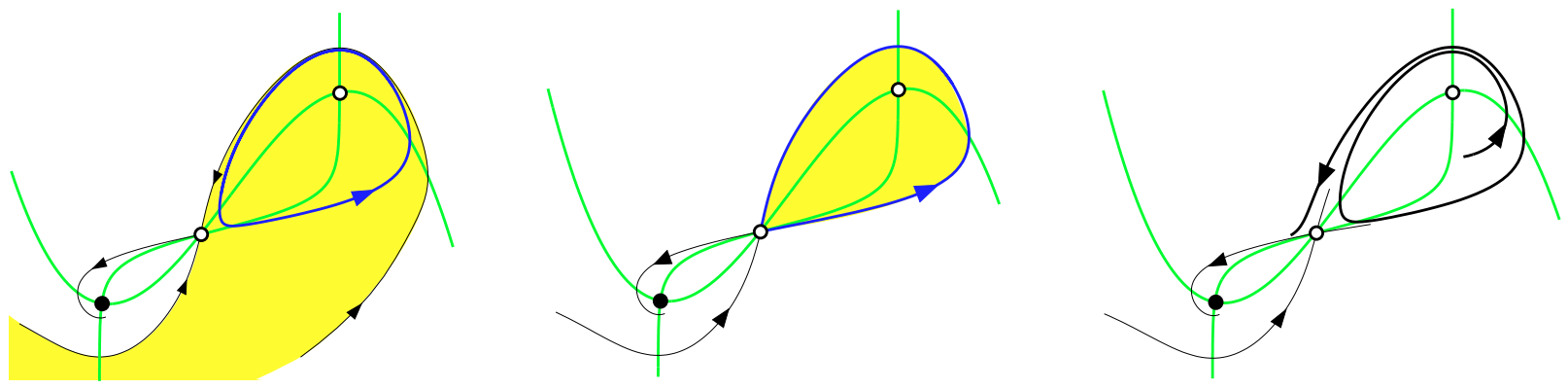

\section{Saddle Homoclinic Orbit}
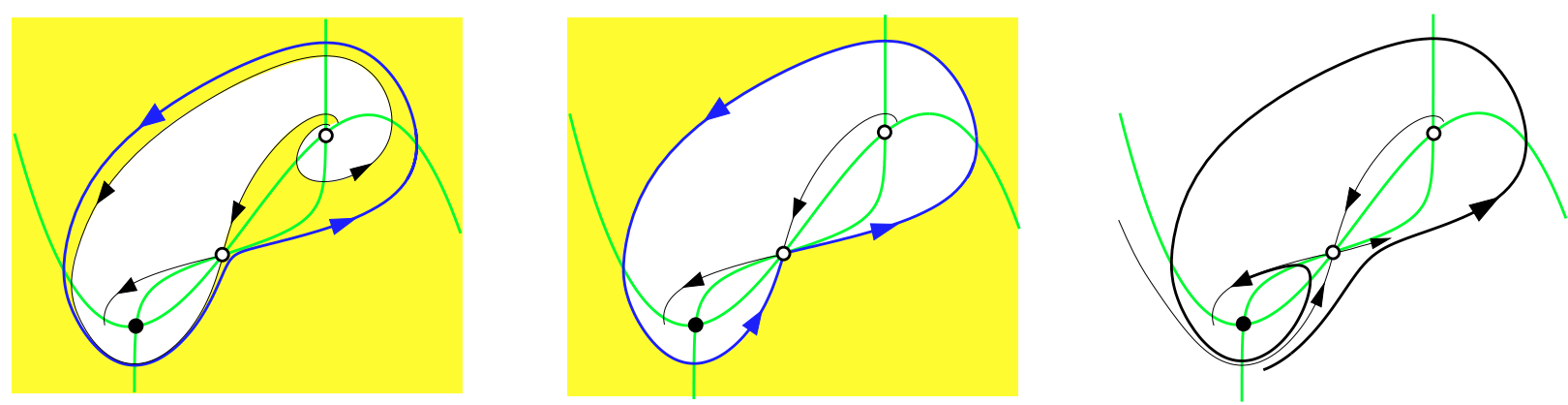

Big Saddle Homoclinic Orbit

Fig. 39. Disappearance of a limit cycle via saddle homoclinic orbit bifurcation in (15). Any perturbation that pushes the solution out of the yellow area would shut down oscillation. 
threshold manifold, and the oscillation can be shut down by an appropriately placed pulse, as we illustrate in Fig. 39. Thus, the two cases differ not only in the asymptotics of spiking frequency, but also in the coexistence of spiking and rest states.

\subsubsection{Spike frequency adaptation}

Slow adaptation processes can lower the frequency of periodic spiking. To illustrate this issue we use the canonical model for Class 1 excitability (4) with an additional adaptation current (5), whose dynamic is depicted in Fig. 40. One can clearly see that the spiking slows down while the adaptation process denoted by $u$ builds up.

In Fig. 41 we change the bifurcation parameter $r$, which has the meaning of injected current, and compare the asymptotic spiking frequency of the canonical model with and without adaptation currents. As one expects, the currents lower significantly the frequency curve.

Wang [1998] observed an interesting phenomenon: The slow adaptation currents seemed to linearize the frequency curve. Later Ermentrout [1998] has proven that this is a general property of all Class 1 spiking systems, i.e. that the infinite slope of the frequency curve at zero becomes finite. Indeed, let $\omega(\lambda)$ describe the dependence of the firing rate on $\lambda$ when there is no adaptation. In our cases $\omega^{\prime}(0)=\infty$. Let $\alpha$ be the amount of nega- tive feedback so that the true firing rate becomes $\omega(\lambda-\alpha)$. The feedback is proportional to the firing rate, i.e.

$$
\alpha=\beta(\omega(\lambda-\alpha))
$$

where $\beta(\omega)$ is some function satisfying

$$
\beta(0)=0 \quad \text { and } \quad \beta^{\prime}(0)>0 .
$$

Following [Ermentrout, 1998] we implicitly differentiate the equation with respect to $\lambda$ to obtain

$$
\frac{d \alpha}{d \lambda}=\beta^{\prime}(\omega(\lambda-\alpha)) \omega^{\prime}(\lambda-\alpha)\left(1-\frac{d \alpha}{d \lambda}\right),
$$

which results in

$$
\frac{d \alpha}{d \lambda}=\frac{\beta^{\prime}(\omega) \omega^{\prime}}{1+\beta^{\prime}(\omega) \omega^{\prime}} \rightarrow 1 \quad \text { as } \lambda \rightarrow 0 .
$$

Hence $\alpha(\lambda) \approx \lambda$, and from (17) we can see that the true firing rate is $\lambda / \beta^{\prime}(0)$. In particular, it has a finite slope $1 / \beta^{\prime}(0)$ at the bifurcation point, see Fig. 42.

As was pointed by Ermentrout [1998], the linearization takes place in some intermediate neighborhood of the bifurcation where the interspike interval is smaller than the adaptation time scale. The method discussed above breaks down at the vicinity of the bifurcation point unless the adaptation is unrealistically slow. In Fig. 41 we magnified
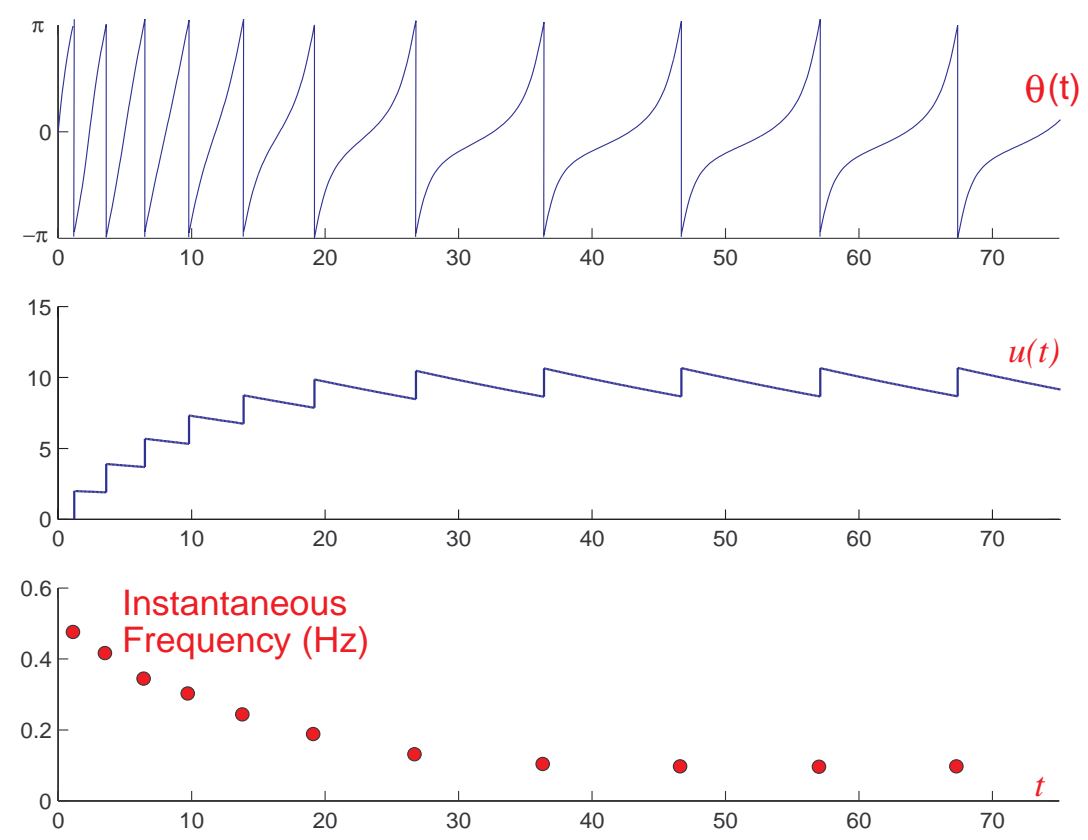

Fig. 40. Slow adaptation processes can change the frequency of repetitive spiking. Shown are simulations of the canonical model $(4,5)$ with $r=2, s=-1 / 5$ and $\eta=1 / 50$. 

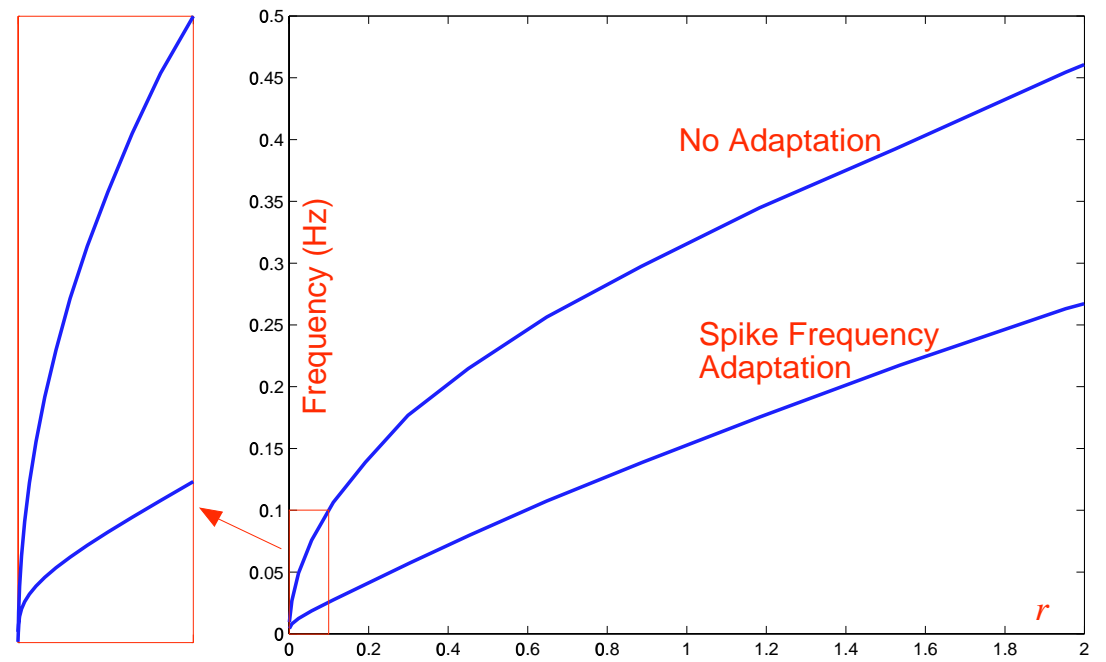

Fig. 41. The frequency/current curve for the canonical model $(4,5)$ from Fig. 40 with $(s=-1 / 10)$ and without $(s=0)$ slow adaptation currents. $\eta=1 / 25$. The rectangle on the left-hand side shows magnification of the origin.
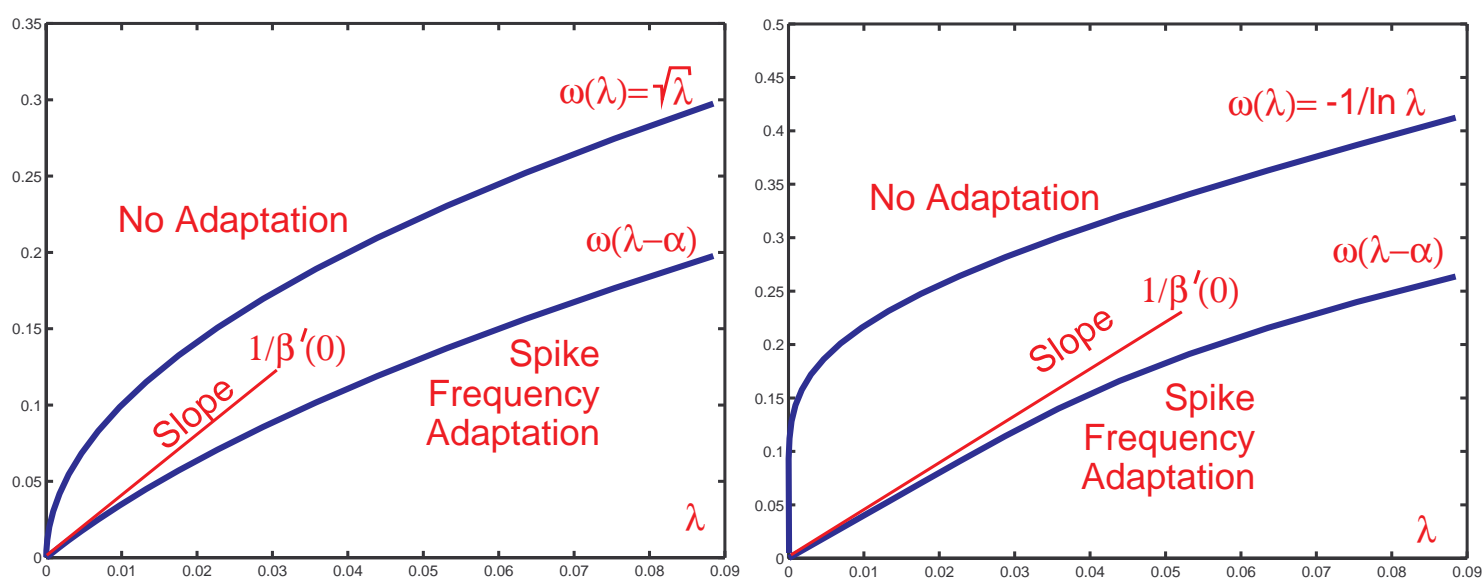

Fig. 42. Spike adaptation can linearize the frequency curves. The function $\beta(\omega)=\omega / 4$ was used to illustrate the issue.

a small neighborhood of the origin to demonstrate that the linearized frequency curve eventually becomes nonlinear with an infinite slope. Thus, adaptation linearizes the frequency curve only in the sense that it pushes nonlinearity closer to the bifurcation point.

\subsection{Class 2 spiking systems}

Whether a limit cycle shrinks to a point via an Andronov-Hopf bifurcation, disappears via a fold limit cycle bifurcation or loses stability via a subcritical flip or Neimark-Sacker bifurcations, its frequency does not vanish. Hence, all these bifurcations result in Class 2 spiking. In the latter two cases there is a well-defined threshold manifold, and the dynamics can be bistable. Thus, an appropriate pulse may shut down the oscillation.

\subsection{Coefficient of variation}

Neurons exhibit random firing patterns when they are subject to random stimulation. The firing randomness can be measured by the coefficient of variation $C_{V}$. Periodic firing has $C_{V}=0$, while Poisson one has $C_{V}=1$. Most cortical neurons have high values of $C_{V}$, while many biophysically detailed Hodgkin-Huxley type models have low ones. This inconsistency is discussed by Softky and Koch [1993]. Gutkin and Ermentrout [1998] showed that the inconsistency may lie in the bifurcation mechanism of generation of action potentials. 
To illustrate the issue, consider the frequency curves in Fig. 36 and suppose that random $I$ fluctuates between the rest and spiking parts. If a system is Class 2 spiking, then it is either silent or fires with a regular interspike interval that is relatively independent from $I$. This leads to low $C_{V}$. In contrast, if the system is Class 1 excitable, then the interspike interval is quite random because it is sensitive to small perturbations of $I$, especially near the bifurcation.

\subsection{Quasi-periodic spiking}

Neural firing can be quasiperiodic or chaotic when the system's dimension is greater than 2 . The former case implies that there is a large amplitude stable invariant torus. Such a torus usually appears and disappears via supercritical Neimark-Sacker bifurcations [Kuznetsov, 1995] of a large amplitude stable limit cycle. In this case the class of spiking is determined by bifurcations of the limit cycle, which we discussed above, and not of the torus.

An interesting case is when the torus is homoclinic to a nonhyperbolic small amplitude limit cycle corresponding to a "subthreshold" oscillation, see Fig. 30. In this case the quasiperiodic firing appears straight from the small limit cycle [Kuznetsov, 1995; Il'iashenko \& Li, 1999]. It has two frequencies: One corresponds to a subthreshold oscillation, it is defined by the frequency of the small limit cycle. The other corresponds to firing, it depends on the distance from bifurcation, $\lambda$, and has the same asymptotic, $\mathcal{O}(\sqrt{\lambda})$, as the one for saddle-node on invariant circle bifurcation or blue-sky catastrophe. Hence such a quasiperiodic spiking belongs to Class 1.

Studying chaotic firing goes beyond the scope of this paper, although we mention chaotic bursting in Sec. 5.3.

\subsection{Phase equations and FM interactions}

Let us consider weakly connected neurons having periodic or quasiperiodic activity

$$
\dot{x}_{i}=f_{i}\left(x_{i}\right)+\varepsilon \sum_{j=1}^{n} g_{i j}\left(x_{i}, x_{j}, \varepsilon\right)
$$

where $\varepsilon \ll 1$ measures the strength of connections. Hoppensteadt and Izhikevich [1997] obtained the estimate $0.004<\varepsilon<0.008$ using experimental data from the hippocampus. Neocortex data suggests 10 times stronger connections.

Dynamics of (18) can be described by a weakly connected phase model

$$
\dot{\vartheta}=\omega_{i}+\varepsilon \sum_{j=1}^{n} h_{i j}\left(\vartheta_{i}, \vartheta_{j}, \varepsilon\right) .
$$

Moreover, one can prove (see [Hoppensteadt \& Izhikevich, 1997, Chap. 9], for exposition of relevant theory) that there is actually a continuous change of variables that transforms (18) into the phase model, thereby making it a canonical model.

Here $\vartheta_{i} \in \mathbb{S}^{1}$ is the phase of oscillation of the $i$ th neuron, and $\omega_{i}$ is its frequency. Each function $h_{i j}$ depends on $f_{i}, f_{j}$, and $g_{i j}$. Its meaning is quite different from that of $g_{i j}$.

- Each function $g_{i j}\left(x_{i}, x_{j}, \varepsilon\right)$ in (18) describes physical connections from the $j$ th to the $i$ th oscillator.

- Each function $h_{i j}\left(\vartheta_{i}, \vartheta_{j}, \varepsilon\right)$ in (19) describes effective connections from the $j$ th to the $i$ th oscillator. That is, it describes how the phase of the $j$ th oscillator affects that of the $i$ th one.

The function $h_{i j}$ has the following interesting property: It is nearly constant and does not depend on $\vartheta_{j}$ unless $\omega_{i}$ and $\omega_{j}$ are low-order quasiresonant [Hoppensteadt \& Izhikevich, 1997, 1998], i.e. unless

$$
\left|p \omega_{i}+q \omega_{j}\right|<\varepsilon
$$

for some small integers $p$ and $q$. This result has been generalized for quasiperiodic oscillators [Izhikevich, 1999a].

A direct consequence of this result is the following: If the frequencies are not resonant, then the phase of the $i$ th oscillator does not depend on the phase of the $j$ th one. Therefore, such oscillators cannot lock; They cannot even influence each other (because $h_{i j}=$ const), even when they are interconnected $\left(g_{i j} \neq\right.$ const). We see that in order to interact, it is not enough to have physical connection between two oscillators, they must also establish a certain low-order resonance relation between their frequencies. We refer to such interactions as being Frequency Modulated (FM).

\subsubsection{Damped subthreshold oscillation}

Let us illustrate the major point of the FM interaction theory using weakly connected neurons near 
Andronov-Hopf bifurcation, which we considered at the end of Sec. 2.2. Each such neuron exhibits damped subthreshold oscillation of membrane potential with some frequency $\omega_{i}$. A canonical model for a weakly connected network (18) of such neurons has the form [Aronson et al., 1990; Hoppensteadt \& Izhikevich, 1996, 1997]

$$
\dot{z}_{i}=\left(\varepsilon a_{i}+\mathrm{i} \omega_{i}\right) z_{i} \pm z_{i}\left|z_{i}\right|^{2}+\varepsilon \sum_{j=1}^{n} c_{i j} z_{j} .
$$

It is similar to (13). The change of variables

$$
z_{i}=\sqrt{\varepsilon} v_{i} e^{\mathrm{i} \omega_{i} t}, \quad v_{i} \in \mathbb{C}, \quad i=1, \ldots, n,
$$

results in the system

$$
\dot{v}_{i}=\varepsilon\left(a_{i} v_{i} \pm v_{i}\left|v_{i}\right|^{2}+\sum_{j=1}^{n} c_{i j} e^{\mathrm{i}\left(\omega_{j}-\omega_{i}\right) t} v_{j}\right),
$$

which could easily be averaged.

If $\omega_{j} \neq \omega_{i}$, then the highly oscillatory term $c_{i j} e^{\mathrm{i}\left(\omega_{j}-\omega_{i}\right) t} v_{j}$ vanishes after averaging, and the variable $v_{j}$ does not participate in the $i$ th equation. Hence the phase of the $j$ th neuron cannot influence that of the $i$ th neuron. Such neurons do not interact even though $x_{j}$ and $x_{i}$ are physically connected provided that $g_{i j} \neq$ const in (18). In contrast, if $\omega_{j}=\omega_{i}$, then

$$
c_{i j} e^{\mathrm{i}\left(\omega_{j}-\omega_{i}\right) t} v_{j}=c_{i j} v_{j}
$$

survives the averaging. Hence the $j$ th variable participates in the $i$ th equation.

We see that the existence of physical connections between two oscillators, which are accounted by the function $g_{i j}\left(x_{i}, x_{j}\right)$, does not guarantee that the oscillators can influence each other. There should also be certain relation between their frequencies, e.g. $\omega_{j}=\omega_{i}+\mathcal{O}(\varepsilon)$, or else the physical connections are not effective.

Having a low-order resonant relation, e.g. $\omega_{j}=2 \omega_{i}$, is not enough here because periodic activity at an Andronov-Hopf bifurcation does not have noticeable harmonics or subharmonics. However, it is usually enough far away from the bifurcation, since the limit cycle becomes distorted and periodic activity acquires significant harmonics and subharmonics.

\subsubsection{Fast subthreshold oscillation}

If a neuron exhibits fast subthreshold oscillation of its membrane potential, then its response to a brief strong input may depend on the amplitude and timing of the input, as we discussed in Sec. 2.4.

Now suppose the input is weak, so that it never evokes an action potential, but can modulate the subthreshold oscillation, e.g. by changing its phase, so that the neuron would react differently to a future strong pulse. From the FM interaction theory it follows that the phase of subthreshold oscillation can be affected only by those neurons that fire with a certain resonant frequency. By changing the frequency of the subthreshold limit cycle, the neuron can control the set of the presynaptic neurons that can modulate its dynamics.

\subsubsection{Spiking oscillations}

Now consider two periodically spiking neurons. From the FM interaction theory it follows that the neurons communicate only when there is a loworder quasiresonance relation between their firing frequencies. Thus, a neuron can turn off and on its connection with the other neurons simply by changing the frequency of spiking without changing the synaptic efficacy. The entire brain can rewire itself dynamically without changing the synaptic hardware.

One can argue that studying periodically spiking neurons could have a questionable biological value because neurons do not fire periodic spike trains. They do, however, when they are in the bursting regime, as we describe in the next section. In Sec. 4.6 we show how the theory of FM interactions helps to understand synchronization properties of weakly connected bursters.

\section{Bursting}

So far we have considered spiking mechanisms assuming that all parameters of the neuron are fixed. From now on we drop this assumption and consider neural systems of the form

$$
\dot{x}=f(x, u),
$$

where $u$ represents slowly changing parameters in the system.

Quasistatic Bifurcation Parameter. We treat $u$ from (20) as being a vector of quasistatic bifurcation parameters that control neural behavior. The 


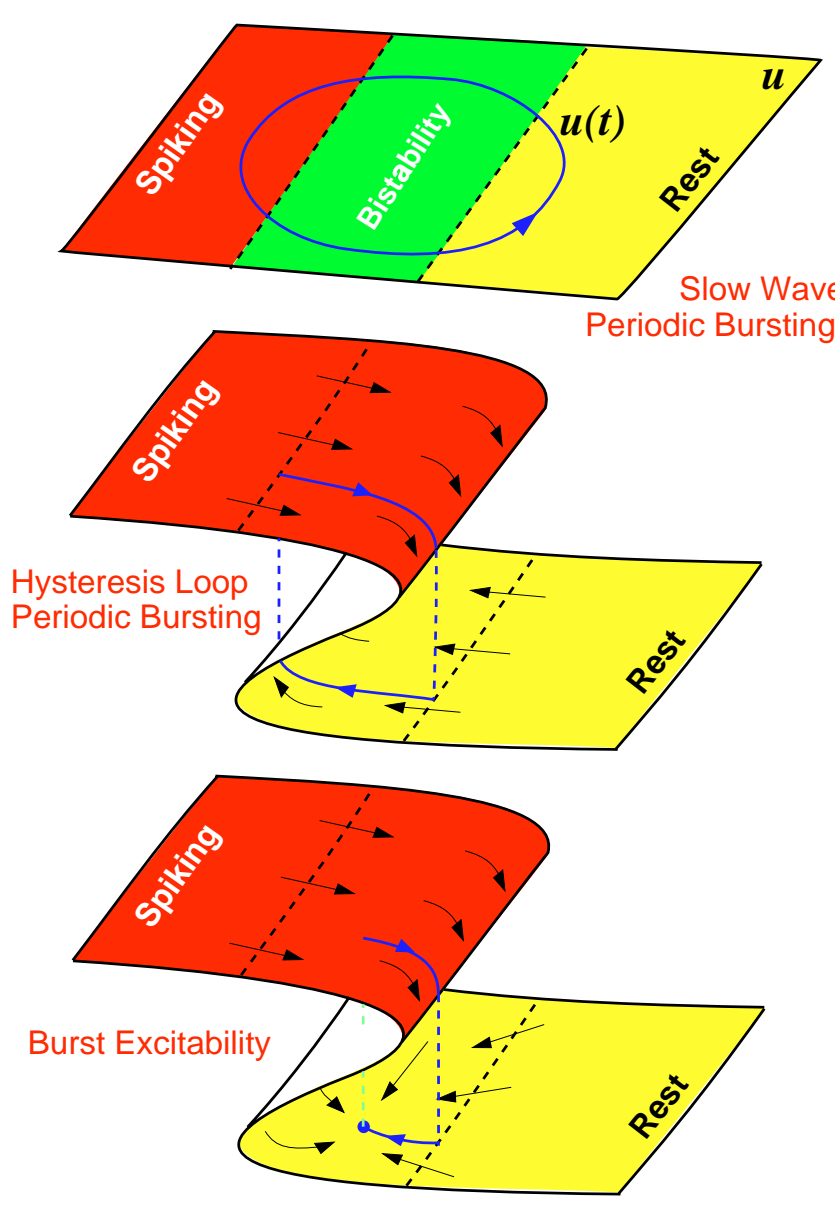

Fig. 43. Parameter $u$ in (20) can control spiking behavior. When $u$ changes slowly, the neuron can exhibit bursting behavior.

neuron can be excitable, bistable, or spiking depending on the value of $u$; see Fig. 43 .

If $u$ visits the spiking and quiescent areas periodically, the neuron exhibits periodic bursting behavior. During each burst the neuron can fire many spikes or just a doublet or a triplet, depending on the period of time parameter $u$ spends in the spiking area relative to the period of a single spike.

There could be many causes for periodic activity of $u$, as shown in Fig. 43. The simplest one is when $u$ has a limit cycle attractor that is (relatively) independent of the value of $x$. We refer to such bursters as slow wave periodic bursters.

In general, $u$ may not have a periodic attractor for any fixed value of $x$. In fact, it could always converge to an equilibrium for all relevant $x$; see Fig. 44. However, the location of the equilibrium may depend on $x$, which would cause periodic bursting.

Usually, the value of $u$ depends strongly on the current state $x(t)$ : Repetitive firing of $x$ pushes $u$
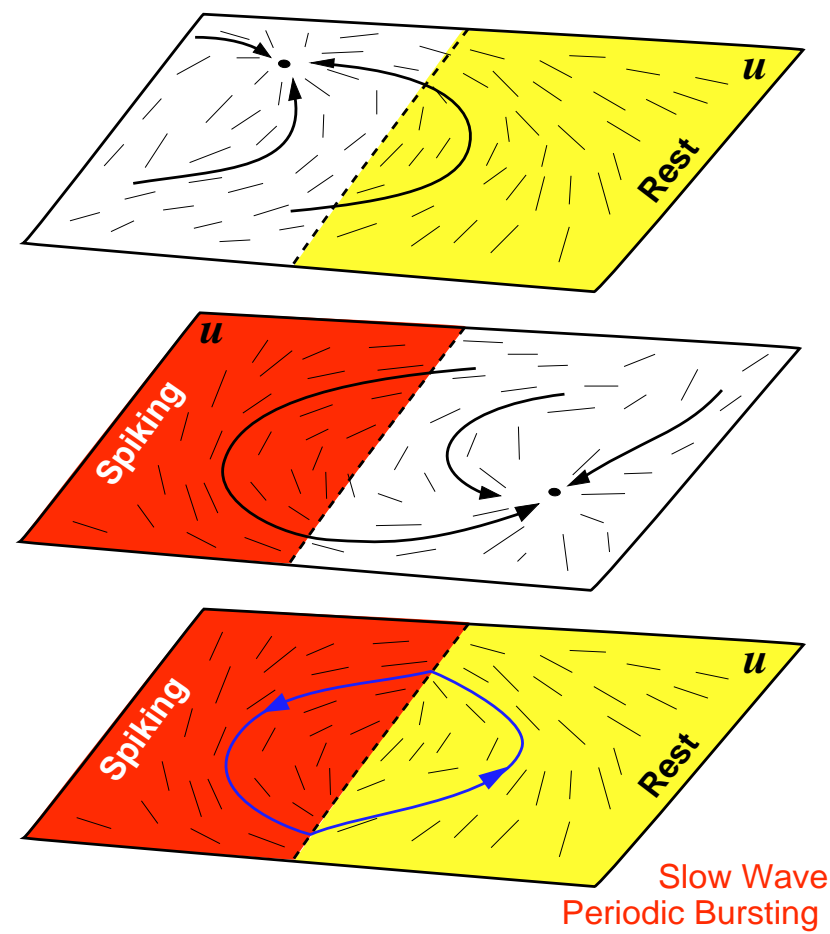

Fig. 44. A neural system may exhibit slow wave periodic bursting even when the parameter $u$ in (20) has an equilibrium dynamics for any fixed $x$.

outside the spiking area, which results in termination of firing. If, in addition, quiescent $x$ pushes $u$ outside the resting area, then $u$ visits the spiking and quiescent areas periodically, and the neuron exhibits hysteresis loop periodic bursting. The parameter $u$ may be a scalar in this case. If quiescent $x$ does not push $u$ beyond the bistable area, then the neuron exhibits burst excitability: It has quiescent excitable dynamics, but its response to perturbations is not a single spike, but a burst of spikes, as we illustrate in Fig. 45.

\subsection{Fast-slow bursting}

We say that a neuron is a fast-slow burster if its bursting behavior can be described by a singularly perturbed system of the form

$$
\begin{array}{ll}
\dot{x}=f(x, u) & \text { (Fast Spiking) } \\
\dot{u}=\mu g(x, u) & \text { (Slow Modulation) }
\end{array}
$$

where $\mu \ll 1$ represents the ratio of time scales between spiking and modulation. Vector $x \in \mathbb{R}^{m}$ describes relatively fast processes associated with generation of action potentials, and $u \in \mathbb{R}^{k}$ describes relatively slow processes that modulate $x$. Considering dynamics of $x$ and $u$ separately constitutes 


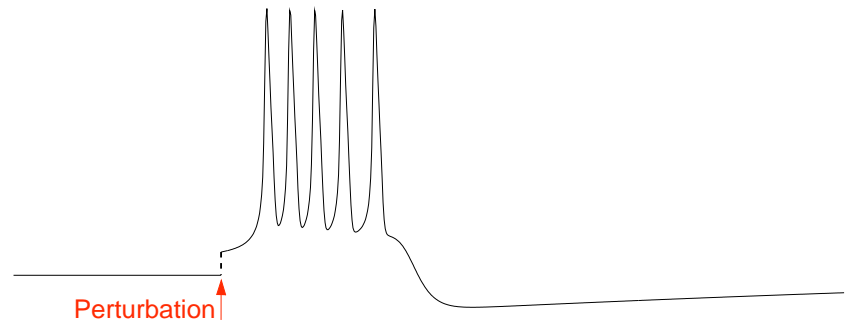

Fig. 45. Burst excitability in the Morris-Lecar model with an additional slow subsystem; see bottom of Fig. 43 .

the method of dissection of neural bursting [Rinzel \& Lee, 1987], or of quasistatic state approximation [Hoppensteadt, 1993].

We say that the burster is " $m+k$ " when the fast subsystem is $m$-dimensional and the slow subsystem is $k$-dimensional. In this paper we provide many examples of " $2+1$ " and " $2+2$ " bursters.

\subsubsection{Classical singular perturbation theory}

Asymptotic theory of singularly perturbed dynamical systems is well-developed [Hoppensteadt, 1993; Mishchenko et al., 1994] and one would expect to find methods to analyze fast-slow bursters of the form (21). However, the basic assumption of the theory that the fast subsystem has only equilibrium dynamics, is violated when the neuron starts to fire periodically. Thus, the entire bulk of theory is helpless in studying fast-slow bursting. (An exception is the Pontryagin's problem, which is related to "fold cycle/fold cycle" bursting; see Sec. 7 in [Mishchenko et al., 1994]).

\subsubsection{Averaging}

One can try to use averaging to get some insight into the behavior of the system (21). This approach was used successfully, e.g. by Rinzel and Lee [1986], Pernarowski et al. [1992], Smolen et al. [1993] and Baer et al. [1995].

Let $x(t)=x(t, u)$ be a solution of the fast subsystem when $u=$ const. Using it in the slow subsystem results in

$$
\dot{u}=\mu g(x(t, u), u),
$$

which can be reduced to

$$
\dot{w}=\mu \bar{g}(w)
$$

by a near-identity change of variables $w=u+o(\mu)$. Here

$$
\bar{g}(w)=\lim _{T \rightarrow \infty} \frac{1}{T} \int_{0}^{T} g(x(t, w), w) d t
$$

is the average of $g$. Unfortunately, many problems may arise when $u$ passes slowly bifurcation values. For example, the period of $x(t, u)$ may go to infinity, as happens in Class 1 systems, or transients may take as long as $1 / \mu$ units of time, or the averaged system (22) is not Lipchitz. In all these cases the straightforward averaging discussed above fails. We provide an example when we discuss "Hopf/Hopf" hysteresis loop bursting.

\subsubsection{Equivalent voltage}

If a neural model exhibits bistable behavior, then the function $\bar{g}$ in (22) is not single-valued, but it has layers (sheets) similar to those depicted in Fig. 43. This is the result of the averaging that removes completely the fast dynamics. To study possible hysteresis behavior we need to retain at least one "fast" variable. This is always possible in the following important class of fast-slow bursters

$$
\begin{aligned}
& \dot{x}=f(x, u) \\
& \dot{u}=\mu g(h(x), u)
\end{aligned}
$$

where $h: \mathbb{R}^{m} \rightarrow \mathbb{R}$ is an arbitrary function, e.g. the membrane voltage at state $x$. Notice that the image of $h$ is a scalar.

If the slow variable is a scalar too, then for any value of $u$ there is a (possibly nonunique) value of another scalar, $v$, such that $g(v, u)=\bar{g}(u)$, where $\bar{g}(u)$ is the averaged function defined above. We refer to $v$ as being the equivalent voltage [Bertram et al., 1995]. It allows to reduce (23) to the twodimensional system of the form

$$
\begin{aligned}
& \dot{v}=s(v, u) \\
& \dot{u}=\mu g(v, u)
\end{aligned}
$$

where $g$ is exactly the same as in (21), and $s: \mathbb{R}^{2} \rightarrow \mathbb{R}$ is some function that can be found numerically. Its set of zeroes often has the $N$-shape depicted in Fig. 46. If (23) describes a hysteresis loop periodic burster, then system (24) is a relaxation oscillator. Thus, most of the results regarding synchronization of coupled relaxation oscillators can be applied to coupled bursters.

\subsection{Classification}

Below we provide a classification scheme for periodic fast-slow bursting. Any such bursting involves 


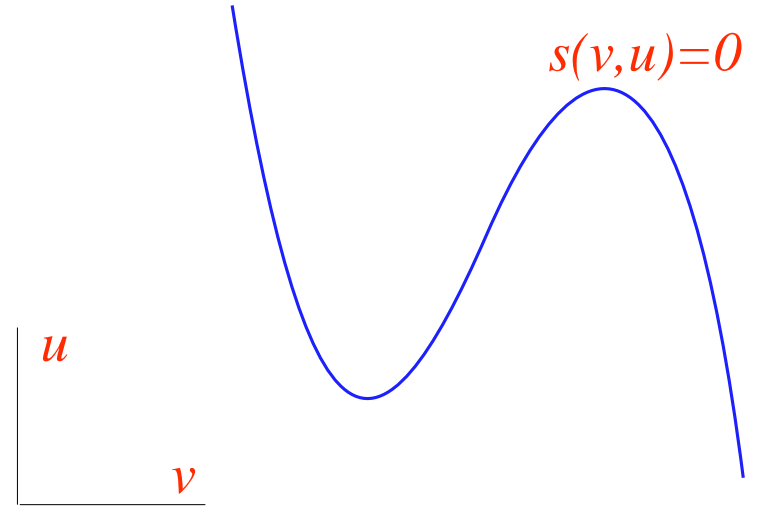

Fig. 46. The set of zeroes of function $s$ in (24) often has an $N$-shape.

at least two bifurcations (see Fig. 2):

- Bifurcation of the quiescent state leading to repetitive spiking.

- Bifurcation of the repetitive spiking leading to the quiescent state.

There could also be other bifurcations, which do not switch between the rest and spiking states. Since there are 12 relevant codimension 1 bifurcations of the quiescent state and 10 relevant codimension 1 bifurcations of the spiking state, there are 120 different types of codimension 1 bursters; see Table 4 .

We refer to a burster as being planar when the fast subsystem is two-dimensional, i.e. the burster is " $2+k$ ". This imposes severe restriction on possible bifurcations. In particular, there are only 24 various types of planar codimension 1 fast-slow bursters, which are summarized in Table 3 . We emphasize planar bursters because they have a greater chance to be encountered in computer simulations.

Various bursters can be combined into groups depending on the nature of quiescent and spiking state at the bifurcations. If the quiescent state is a stable equilibrium and the spiking state is a limit cycle attractor, then we say that the burster is point-cycle. A cycle-cycle burster occurs when the quiescent state is a small amplitude, "subthreshold" limit cycle attractor. Similarly, point-torus and cycle-torus bursters are when the spiking state is quasiperiodic. A point-point bursting is discussed in Sec. 4.5. It occurs when the parameter $\mu$ in (21) is not small enough. A cycle-point bursting is also feasible, but we do not discuss it here.

\subsubsection{What is the name?}

We face a challenging problem: How to label all those bursters? We want self-explanatory names that are easy to remember and understand. Thus, we should not number them [Bertram et al., 1995], since it would lead, e.g. to bursters of Type XXVII, Type LXIII, Type LCXVI, etc. We cannot use descriptions such as "elliptic", "parabolic", "hyperbolic", "triangular", "rectangular", etc., since they are misleading. Instead, we base our nomenclature on the names of the two bifurcations involved. Still, many bifurcations have multiple names. For example, a fold bifurcation is also known as saddle-node, tangent, or limit point bifurcation. Whenever possible we choose the shortest name. For example, we refer to the saddle-node on invariant circle bifurcation (also known as fold or saddle-node on limit cycle) as just a circle bifurcation, partially because it is the only codimension 1 bifurcation of a circle flow. We refer to an Andronov-Hopf bifurcation, also known as Poincaré-Andronov-Hopf, as just Hopf when it is supercritical and subHopf when it is subcritical. Our results are summarized in Table 4. For example, the famous "square-wave" (Type I) burster is a "fold/homoclinic" burster according to our classification, because the rest state disappears via fold bifurcation and the limit cycle attractor disappears via saddle homoclinic orbit bifurcation; see Fig. 59.

\subsection{Planar point-cycle bursting}

In this section we give examples of planar pointcycle bursters. For this we add slow subsystems to some well-known neural models, such as MorrisLecar, that have sufficiently rich bifurcation structure. The easiest way to get bursting is to take a two-dimensional slow subsystem having a "slow wave" (subcellular) limit cycle attractor that intersects the desired bifurcation curves of a fast (spiking) subsystem. All such bursters are " $2+2$ ". This is a classical approach to obtain the "circle/circle" ("parabolic") bursting [Ermentrout \& Kopell, 1986a, 1986b]. We show, however, that each burster can be of hysteresis loop type with only one slow variable, although finding an example of such " $2+1$ " bursting is more difficult.

For the sake of convenience, we provide a list of references to relevant illustrations in Table 5 .

\subsubsection{Slow wave bursting}

Consider the four types of planar point-cycle bursting that are depicted in the upper left corner of Table 4. All of them can be generated by a 
Table 4. A classification of codimension 1 fast-slow bursters. See also Figs. 53 and 126.

\begin{tabular}{|c|c|c|c|c|c|c|c|c|c|c|}
\hline Bifurcations & $\begin{array}{c}\text { Saddle-Node } \\
\text { on Invariant } \\
\text { Circle }\end{array}$ & $\begin{array}{c}\text { Saddle } \\
\text { Homoclinic } \\
\text { Orbit }\end{array}$ & $\begin{array}{c}\text { Supercritical } \\
\text { Andronov-Hopf }\end{array}$ & $\begin{array}{l}\text { Fold } \\
\text { Limit } \\
\text { Cycle }\end{array}$ & $\begin{array}{l}\text { Subcritical } \\
\text { Flip }\end{array}$ & $\begin{array}{c}\text { Subcritical } \\
\text { Neimark-Sacker }\end{array}$ & $\begin{array}{c}\text { Saddle-Focus } \\
\text { Homoclinic } \\
\text { Orbit }\end{array}$ & $\begin{array}{c}\text { Focus-Focus } \\
\text { Homoclinic } \\
\text { Orbit }\end{array}$ & $\begin{array}{c}\text { Blue-sky } \\
\text { Catastrophe }\end{array}$ & $\begin{array}{c}\text { Fold Cycle on } \\
\text { Homoclinic } \\
\text { Torus }\end{array}$ \\
\hline Fold & $\begin{array}{l}\text { fold/ } \\
\text { circle }\end{array}$ & $\begin{array}{c}\text { fold/ } \\
\text { homoclinic }\end{array}$ & $\begin{array}{l}\text { fold/ } \\
\text { Hopf }\end{array}$ & $\begin{array}{c}\text { fold/ } \\
\text { fold cycle }\end{array}$ & $\begin{array}{l}\text { fold/ } \\
\text { flip }\end{array}$ & $\begin{array}{l}\text { fold/ } \\
\text { Sacker }\end{array}$ & $\begin{array}{c}\text { fold/ } \\
\text { saddle-focus } \\
\text { homoclinic }\end{array}$ & $\begin{array}{l}\text { fold/ } \\
\text { focus-focus } \\
\text { homoclinic }\end{array}$ & $\begin{array}{c}\text { fold/ } \\
\text { blue-sky }\end{array}$ & $\begin{array}{l}\text { fold/ } \\
\text { torus }\end{array}$ \\
\hline $\begin{array}{l}\text { Saddle-Node } \\
\text { on Invariant } \\
\text { Circle }\end{array}$ & $\begin{array}{l}\text { circle/ } \\
\text { circle }\end{array}$ & $\begin{array}{c}\text { circle/ } \\
\text { homoclinic }\end{array}$ & $\begin{array}{l}\text { circle/ } \\
\text { Hopf }\end{array}$ & $\begin{array}{c}\text { circle/ } \\
\text { fold cycle }\end{array}$ & $\begin{array}{l}\text { circle/ } \\
\text { flip }\end{array}$ & $\begin{array}{l}\text { circle/ } \\
\text { Sacker }\end{array}$ & $\begin{array}{c}\text { circle/ } \\
\text { saddle-focus } \\
\text { homoclinic }\end{array}$ & $\begin{array}{l}\text { circle/ } \\
\text { focus-focus } \\
\text { homoclinic }\end{array}$ & $\begin{array}{l}\text { circle/ } \\
\text { blue-sky }\end{array}$ & $\begin{array}{l}\text { circle/ } \\
\text { torus }\end{array}$ \\
\hline $\begin{array}{l}\text { Supercritical } \\
\text { Andronov- } \\
\text { Hopf }\end{array}$ & $\begin{array}{l}\text { Hopf/ } \\
\text { circle }\end{array}$ & $\begin{array}{c}\text { Hopf/ } \\
\text { homoclinic }\end{array}$ & $\begin{array}{l}\text { Hopf/ } \\
\text { Hopf }\end{array}$ & $\begin{array}{l}\text { Hopf/ } \\
\text { fold cycle }\end{array}$ & $\begin{array}{l}\text { Hopf/ } \\
\text { flip }\end{array}$ & $\begin{array}{l}\text { Hopf/ } \\
\text { Sacker }\end{array}$ & $\begin{array}{c}\text { Hopf/ } \\
\text { saddle-focus } \\
\text { homoclinic }\end{array}$ & $\begin{array}{l}\text { Hopf/ } \\
\text { focus-focus } \\
\text { homoclinic }\end{array}$ & $\begin{array}{c}\text { Hopf/ } \\
\text { blue-sky }\end{array}$ & $\begin{array}{l}\text { Hopf/ } \\
\text { torus }\end{array}$ \\
\hline $\begin{array}{l}\text { Subcritical } \\
\text { Andronov- } \\
\text { Hopf }\end{array}$ & $\begin{array}{l}\text { subHopf/ } \\
\text { circle }\end{array}$ & $\begin{array}{l}\text { subHopf/ } \\
\text { homoclinic }\end{array}$ & $\begin{array}{l}\text { subHopf/ } \\
\text { Hopf }\end{array}$ & $\begin{array}{l}\text { subHopf/ } \\
\text { fold cycle }\end{array}$ & $\begin{array}{l}\text { subHopf/ } \\
\text { flip }\end{array}$ & $\begin{array}{l}\text { subHopf/ } \\
\text { Sacker }\end{array}$ & $\begin{array}{l}\text { subHopf/ } \\
\text { saddle-focus } \\
\text { homoclinic }\end{array}$ & $\begin{array}{l}\text { subHopf/ } \\
\text { focus-focus } \\
\text { homoclinic }\end{array}$ & $\begin{array}{l}\text { subHopf/ } \\
\text { blue-sky }\end{array}$ & $\begin{array}{l}\text { subHopf/ } \\
\text { torus }\end{array}$ \\
\hline $\begin{array}{l}\text { Fold } \\
\text { Limit } \\
\text { Cycle }\end{array}$ & $\begin{array}{l}\text { fold cycle/ } \\
\text { circle }\end{array}$ & $\begin{array}{l}\text { fold cycle/ } \\
\text { homoclinic }\end{array}$ & $\begin{array}{c}\text { fold cycle/ } \\
\text { Hopf }\end{array}$ & $\begin{array}{l}\text { fold cycle/ } \\
\text { fold cycle }\end{array}$ & $\begin{array}{l}\text { fold cycle/ } \\
\text { flip }\end{array}$ & $\begin{array}{l}\text { fold cycle/ } \\
\text { Sacker }\end{array}$ & $\begin{array}{l}\text { fold cycle/ } \\
\text { saddle-focus } \\
\text { homoclinic }\end{array}$ & $\begin{array}{l}\text { fold cycle/ } \\
\text { focus-focus } \\
\text { homoclinic }\end{array}$ & $\begin{array}{l}\text { fold cycle/ } \\
\text { blue-sky }\end{array}$ & $\begin{array}{l}\text { fold cycle/ } \\
\text { torus }\end{array}$ \\
\hline $\begin{array}{l}\text { Saddle } \\
\text { Homoclinic } \\
\text { Orbit }\end{array}$ & $\begin{array}{l}\text { homoclinic/ } \\
\text { circle }\end{array}$ & $\begin{array}{l}\text { homoclinic/ } \\
\text { homoclinic }\end{array}$ & $\begin{array}{l}\text { homoclinic/ } \\
\text { Hopf }\end{array}$ & $\begin{array}{l}\text { homoclinic/ } \\
\text { fold cycle }\end{array}$ & $\begin{array}{c}\text { homoclinic/ } \\
\text { flip }\end{array}$ & $\begin{array}{l}\text { homoclinic/ } \\
\text { Sacker }\end{array}$ & $\begin{array}{l}\text { homoclinic/ } \\
\text { saddle-focus } \\
\text { homoclinic }\end{array}$ & $\begin{array}{l}\text { homoclinic/ } \\
\text { focus-focus } \\
\text { homoclinic }\end{array}$ & $\begin{array}{l}\text { homoclinic/ } \\
\text { blue-sky }\end{array}$ & $\begin{array}{l}\text { homoclinic/ } \\
\text { torus }\end{array}$ \\
\hline
\end{tabular}




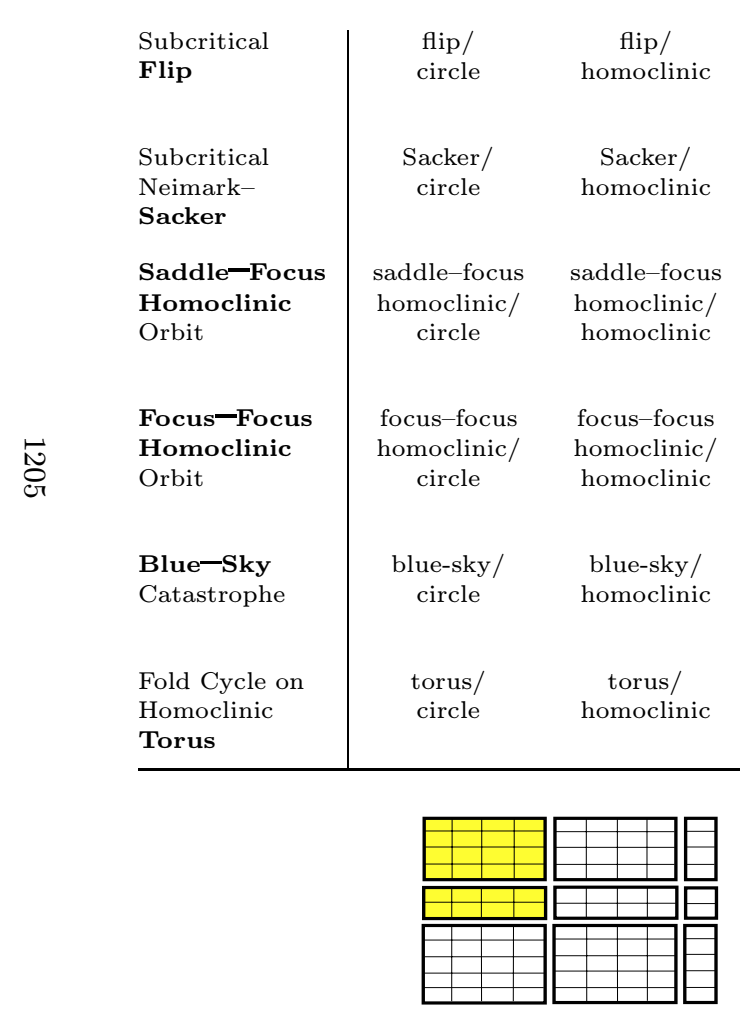

Planar

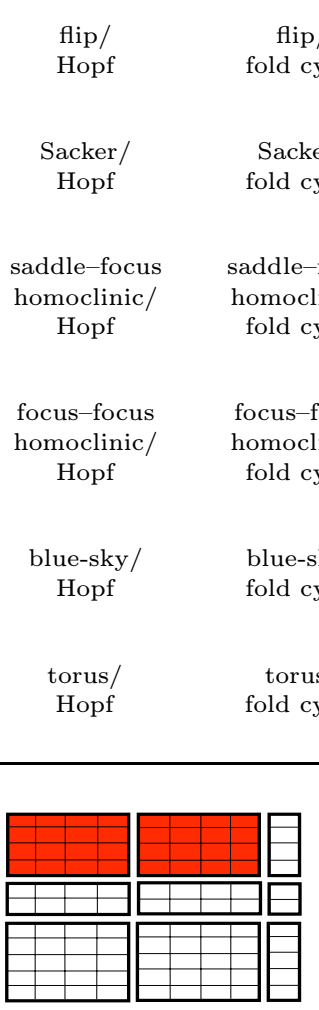

Point-Cycle

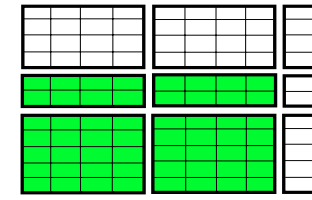

Cycle-Cycle

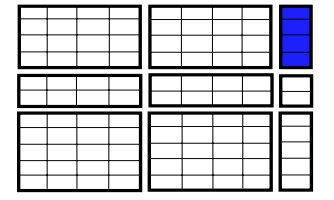

Point-Torus

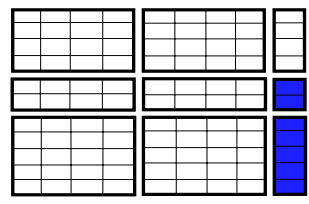

Cycle-Torus

\begin{tabular}{|c|c|c|c|}
\hline $\begin{array}{c}\text { flip/ } \\
\text { saddle-focus } \\
\text { homoclinic }\end{array}$ & $\begin{array}{c}\text { flip/ } \\
\text { focus-focus } \\
\text { homoclinic }\end{array}$ & $\begin{array}{c}\text { flip/ } \\
\text { blue-sky }\end{array}$ & $\begin{array}{l}\text { flip/ } \\
\text { torus }\end{array}$ \\
\hline $\begin{array}{c}\text { Sacker/ } \\
\text { saddle-focus } \\
\text { homoclinic }\end{array}$ & $\begin{array}{c}\text { Sacker/ } \\
\text { focus-focus } \\
\text { homoclinic }\end{array}$ & $\begin{array}{l}\text { Sacker/ } \\
\text { blue-sky }\end{array}$ & $\begin{array}{l}\text { Sacker/ } \\
\text { torus }\end{array}$ \\
\hline $\begin{array}{l}\text { saddle-focus } \\
\text { homoclinic/ } \\
\text { saddle-focus } \\
\text { homoclinic }\end{array}$ & $\begin{array}{l}\text { saddle-focus } \\
\text { homoclinic/ } \\
\text { focus-focus } \\
\text { homoclinic }\end{array}$ & $\begin{array}{l}\text { saddle-focus } \\
\text { homoclinic/ } \\
\text { blue-sky }\end{array}$ & $\begin{array}{l}\text { saddle-focus } \\
\text { homoclinic/ } \\
\text { torus }\end{array}$ \\
\hline $\begin{array}{l}\text { focus-focus } \\
\text { homoclinic/ } \\
\text { saddle-focus } \\
\text { homoclinic }\end{array}$ & $\begin{array}{l}\text { focus-focus } \\
\text { homoclinic/ } \\
\text { focus-focus } \\
\text { homoclinic }\end{array}$ & $\begin{array}{l}\text { focus-focus } \\
\text { homoclinic/ } \\
\text { blue-sky }\end{array}$ & $\begin{array}{c}\text { homoclinic/ } \\
\text { torus }\end{array}$ \\
\hline $\begin{array}{c}\text { blue-sky/ } \\
\text { saddle-focus } \\
\text { homoclinic }\end{array}$ & $\begin{array}{l}\text { blue-sky/ } \\
\text { focus-focus } \\
\text { homoclinic }\end{array}$ & $\begin{array}{l}\text { blue-sky/ } \\
\text { blue-sky }\end{array}$ & $\begin{array}{c}\text { blue-sky/ } \\
\text { torus }\end{array}$ \\
\hline $\begin{array}{c}\text { torus/ } \\
\text { saddle-focus } \\
\text { homoclinic }\end{array}$ & $\begin{array}{c}\text { torus/ } \\
\text { focus-focus } \\
\text { homoclinic }\end{array}$ & $\begin{array}{c}\text { torus/ } \\
\text { blue-sky }\end{array}$ & $\begin{array}{l}\text { torus/ } \\
\text { torus }\end{array}$ \\
\hline
\end{tabular}


Table 5. A list of figure numbers.

\begin{tabular}{|c|c|c|c|c|}
\hline Bifurcations & $\begin{array}{c}\text { Saddle-Node } \\
\text { on Invariant } \\
\text { Circle }\end{array}$ & $\begin{array}{c}\text { Saddle } \\
\text { Homoclinic } \\
\text { Orbit }\end{array}$ & $\begin{array}{c}\text { Supercritical } \\
\text { Andronov-Hopf }\end{array}$ & $\begin{array}{c}\text { Fold } \\
\text { Limit } \\
\text { Cycle }\end{array}$ \\
\hline Fold & $47,61,87$ & $47,59,60,86$ & 63 & 68,87 \\
\hline $\begin{array}{l}\text { Saddle-Node } \\
\text { on Invariant } \\
\text { Circle }\end{array}$ & $\begin{array}{c}47,49 \\
52,56,57 \\
58,87\end{array}$ & $47,62,87$ & 67 & $64,65,66$ \\
\hline $\begin{array}{l}\text { Supercritical } \\
\text { Andronov-Hopf }\end{array}$ & 72 & 71 & $\begin{array}{l}48,83 \\
84,85\end{array}$ & 48,75 \\
\hline $\begin{array}{l}\text { Subcritical } \\
\text { Andronov-Hopf }\end{array}$ & $73,74,87$ & $69,70,87$ & $48,76,77$ & $\begin{array}{l}48,78 \\
80,82\end{array}$ \\
\hline $\begin{array}{l}\text { Fold Limit } \\
\text { Cycle }\end{array}$ & 98 & 97 & 99 & $93,94,96$ \\
\hline $\begin{array}{l}\text { Saddle } \\
\text { Homoclinic } \\
\text { Orbit }\end{array}$ & 92 & 88,89 & 90 & 92 \\
\hline
\end{tabular}

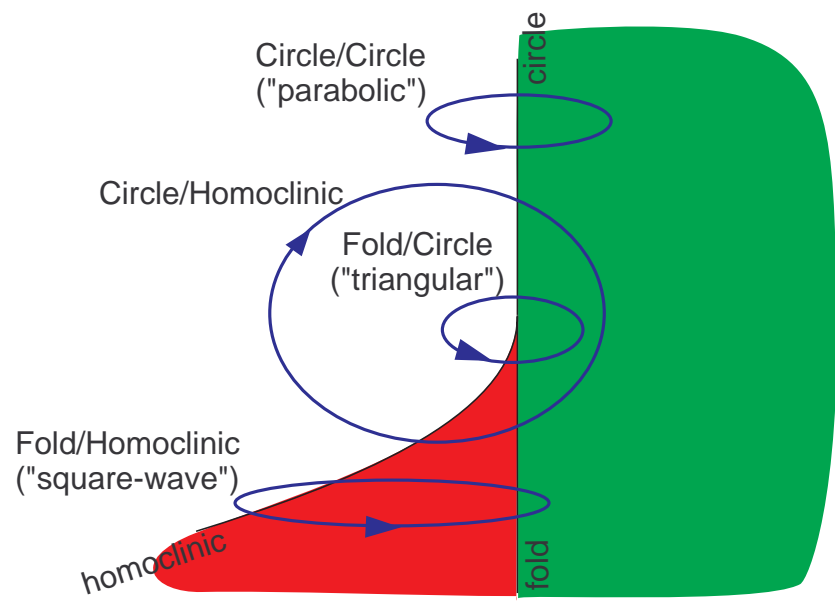

Fig. 47. A neural system near saddle-node separatrix loop bifurcation (see Fig. 22) can exhibit four different types of fast-slow bursting.

system near a saddle-node separatrix loop bifurcation that we discussed in Sec. 2.3. Indeed, the two-dimensional vector of bifurcation parameters can slowly cross the bifurcation curves depicted in Fig. 22. The type of bursting would depend on the location of the slow limit cycle; see Fig. 47.

Similarly, a system near a Bautin bifurcation can exhibit four types of bursting when the bifurcation parameters $a$ and $b$ in topological normal form (12) change slowly; see Fig. 48.

The figures illustrate an important issue: Many bursters, such as "circle/homoclinic", "fold/circle", "Hopf/fold cycle", and "subHopf/Hopf", may involve more than two bifurcations. For example, the "circle/homoclinic" bursting from Fig. 47 exhibits a fold bifurcation during the spiking stage. The bifurcation does not turn the spiking off, but it gives birth to a stable rest state and a saddle, which will participate in the saddle homoclinic orbit bifurcation that turns the spiking off.

Since there can be many other bifurcations of the quiescent and spiking state besides those responsible for onset and termination of the spiking phase, the latter two do not define the type of bursting uniquely, but rather specify a large class, which may have subtypes.

\subsubsection{Hysteresis loop bursting}

The vector of bifurcation parameters in Figs. 47 and 48 has a slow limit cycle attractor. Therefore all those bursters are of the slow wave type. One can easily make the "fold/homoclinic" and the "subHopf/fold cycle" bursters to be of hysteresis loop type by taking advantage of the bistability of attractors. 

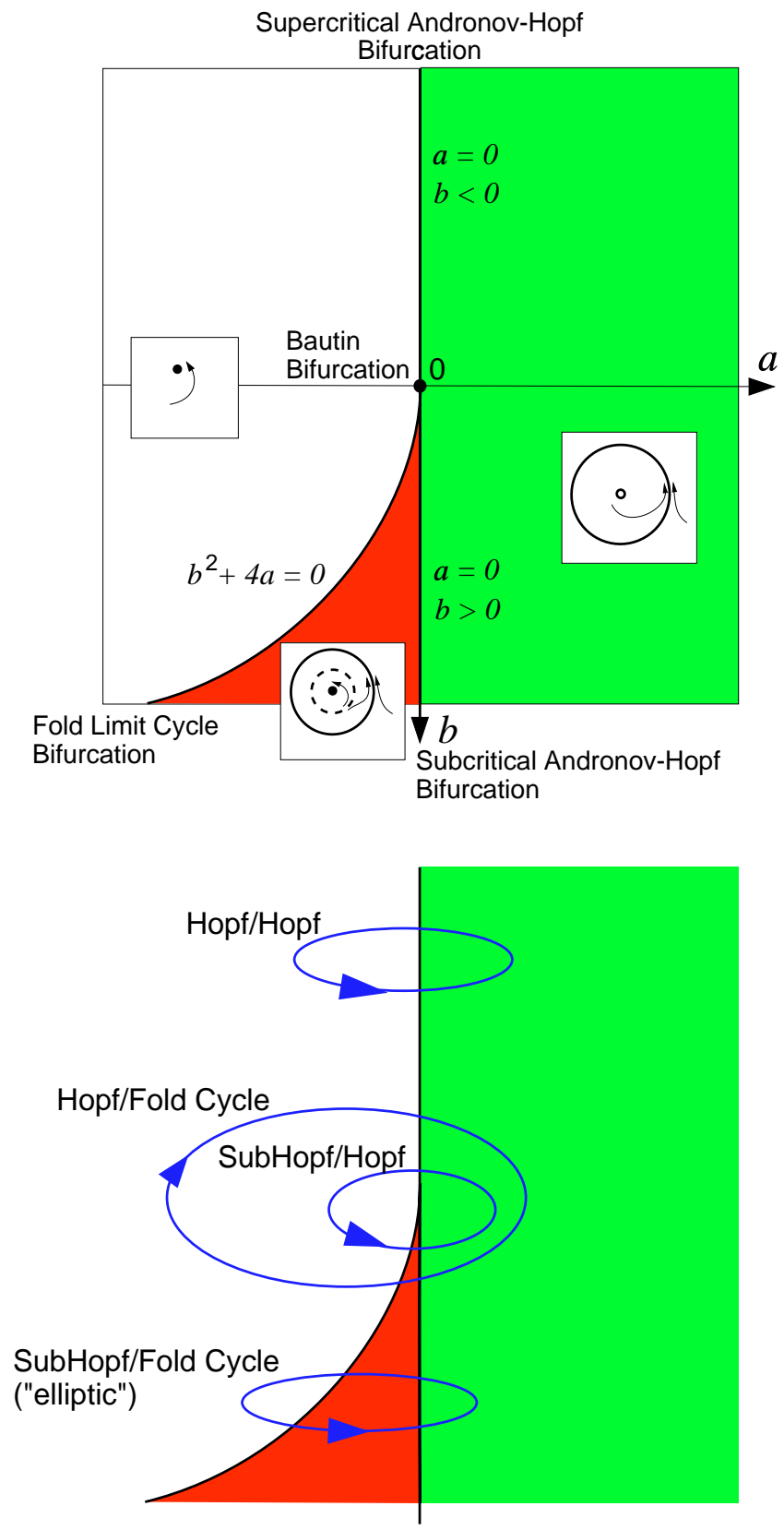

Fig. 48. A neural system near Bautin bifurcation can exhibit four different types of fast-slow bursting.

Contrary to the biophysical folklore, any burster can be of hysteresis loop type with onedimensional slow variable. Indeed, consider the fast-slow hysteresis loop depicted on the left-hand side of Fig. 49. It is customary to refer to the upper (lower) branch as being the up-state (down-state) of a neuron [Wilson, 1993; Wilson \& Kawaguchi, 1996]; see Fig. 50. While the fast variable moves along the up-state, the slow variable may cross some bifurcation curves so that the equilibrium may bifurcate into a limit cycle, and then back to the

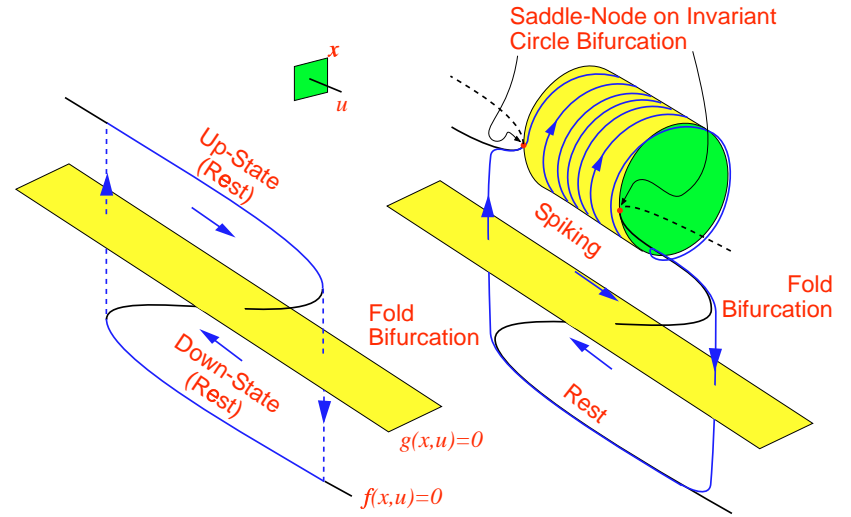

Fig. 49. "Circle/circle" ("parabolic") hysteresis loop periodic bursting with one-dimensional slow variable: The rest (up-)state disappears via saddle-node on invariant circle bifurcation; the spiking limit cycle disappears via another saddle-node on invariant circle bifurcation; the hysteresis behavior occurs due to the coexistence of the up- and downstates. See also Fig. 49.

equilibrium, thereby producing a burst of spikes; see Fig. 51. We depicted the well-known "circle/circle" ("parabolic") bursting on the right-hand side of Fig. 49 to illustrate the issue. The bursting is not of slow wave type, but of hysteresis loop type (" $2+1$ "); see also Fig. 52. In Fig. 53 we show that all planar point-cycle bursters can be of hysteresis loop type.

\subsubsection{Point-Point hysteresis loops}

We refer to the hysteresis loop depicted in Fig. 49 as being a point-point hysteresis loop because it consists of two branches of equilibria corresponding to the up-state and down-state. Such a two-state behavior is exhibited by planar systems having nullclines intersected as in Fig. 54. Notice that the fast nullcline is not cubic but quintic; that is, it has two local maxima and minima. This usually reflects fast activation dynamics of two inward currents having different activation thresholds, e.g. transient low-threshold calcium current and the orthodox sodium current [Rush \& Rinzel, 1994] or two different calcium currents. Such a system can have five equilibria.

The transitions between the up- and downstates do not have to occur exclusively via fold bifurcations. They can also occur via subcritical Andronov-Hopf bifurcations. Hence, there are four different point-point hysteresis loops, which we summarize in Fig. 55. One can modify the dynamics on the upper branch so that it has a window of periodic spiking activity, which would lead to a 


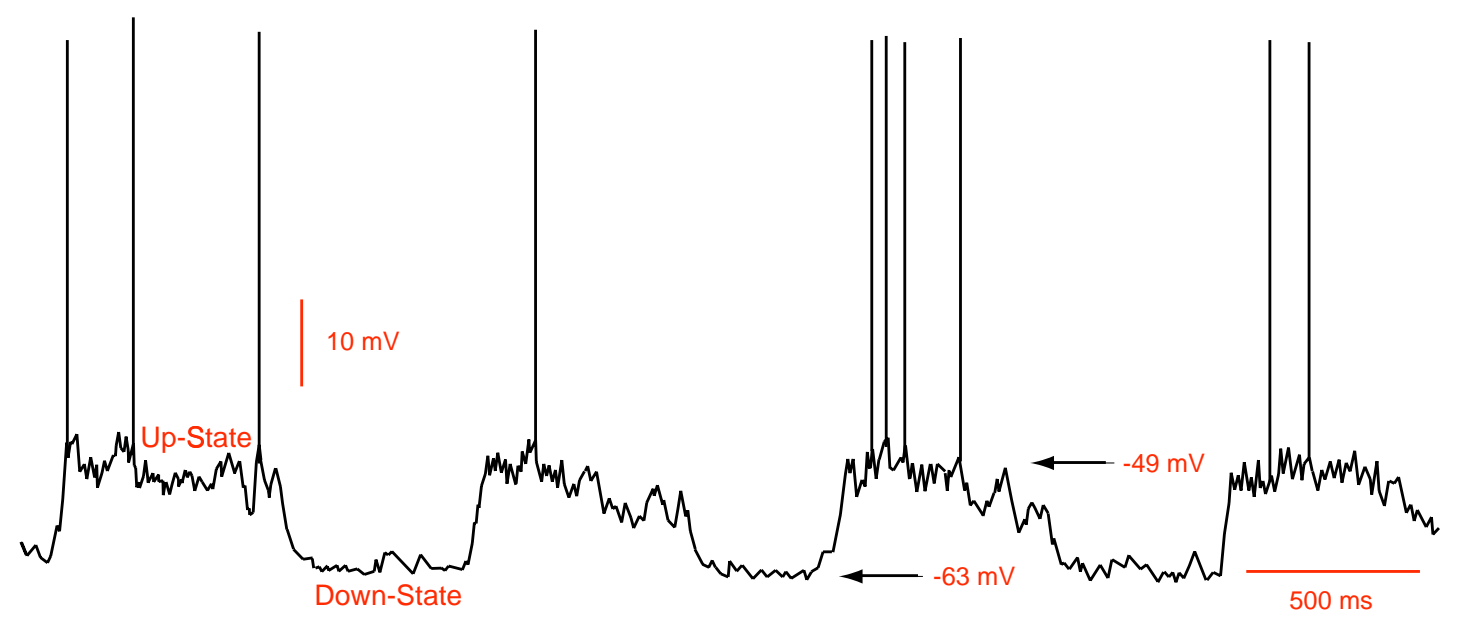

Fig. 50. Intracellular recordings of spontaneous activity of neostriatal spiny cells reveal two-state behavior. Notice that only the up-state is excitable. (Courtesy of Charles Wilson, University of Tennessee.)
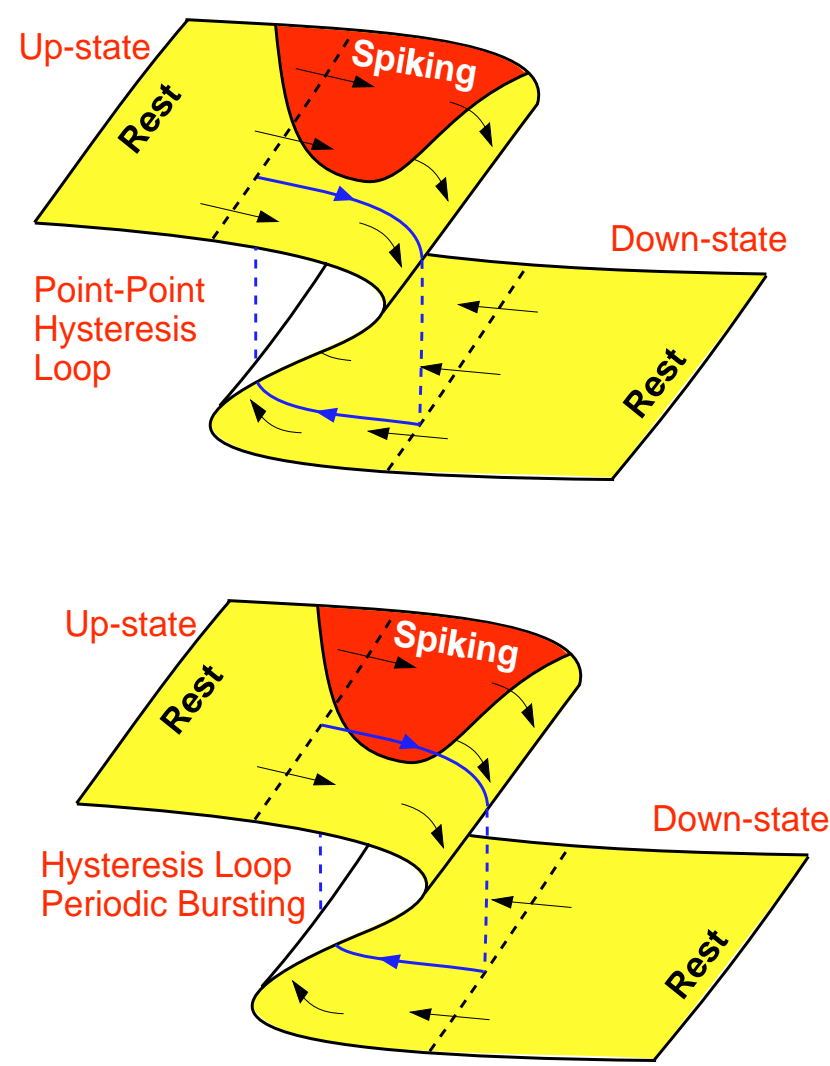

Fig. 51. Modification of dynamics on the upper branch of the point-point hysteresis loop: The slow variable crosses bifurcation curves, creates a window of periodic spiking activity, and results in bursting behavior.

hysteresis loop bursting, as in Fig. 51. For example, one can put two "circle" bifurcations on the upper branch of any of the hysteresis loops, as we do in Fig. 56, and get four different subtypes of the "circle/circle" bursting. Similarly, one can have four subtypes of "circle/Hopf", "Hopf/circle", and "Hopf/Hopf" hysteresis loop bursters.

A remarkable fact is that the point-point hysteresis loops themselves can exhibit bursting behavior despite the fact that there are no stable limit cycles. This happens when the small parameter $\mu$ in (21) is not small enough, as we discuss in Sec. 4.5.

Considering point-point hysteresis loops raises the following issue: The spiking state during the active phase of bursting might be irrelevant to what the cell is doing. For example, the pancreatic $\beta$ cells secrete insulin while they are in the up-state regardless whether their membranes produce spikes or not. Similarly, neurons without axons, such as granule cells in the olfactory bulb, or with short axons, such as many interneurons, may not need spikes to activate synaptic transmission. A mere transition to the up-state may be enough to trigger release of neurotransmitter. Even if such cells exhibit bursting dynamics, their essential neurocomputational features might depend only on the type of the hysteresis loop. In contrast, if a bursting neuron has a long axon, then it might need propagating spikes to trigger synaptic transmission in distant synapses. In this case it is important to understand the mechanism of initiation and termination of repetitive spiking during the burst.

\subsection{4. "Circle/circle" ("parabolic") bursting}

The transition from quiescent state to repetitive spiking and back occurs via a saddle-node on invariant circle bifurcation; see Fig. 47. Since the frequency of emerging and terminating spiking 


\section{"Circle/Circle" Bursting}
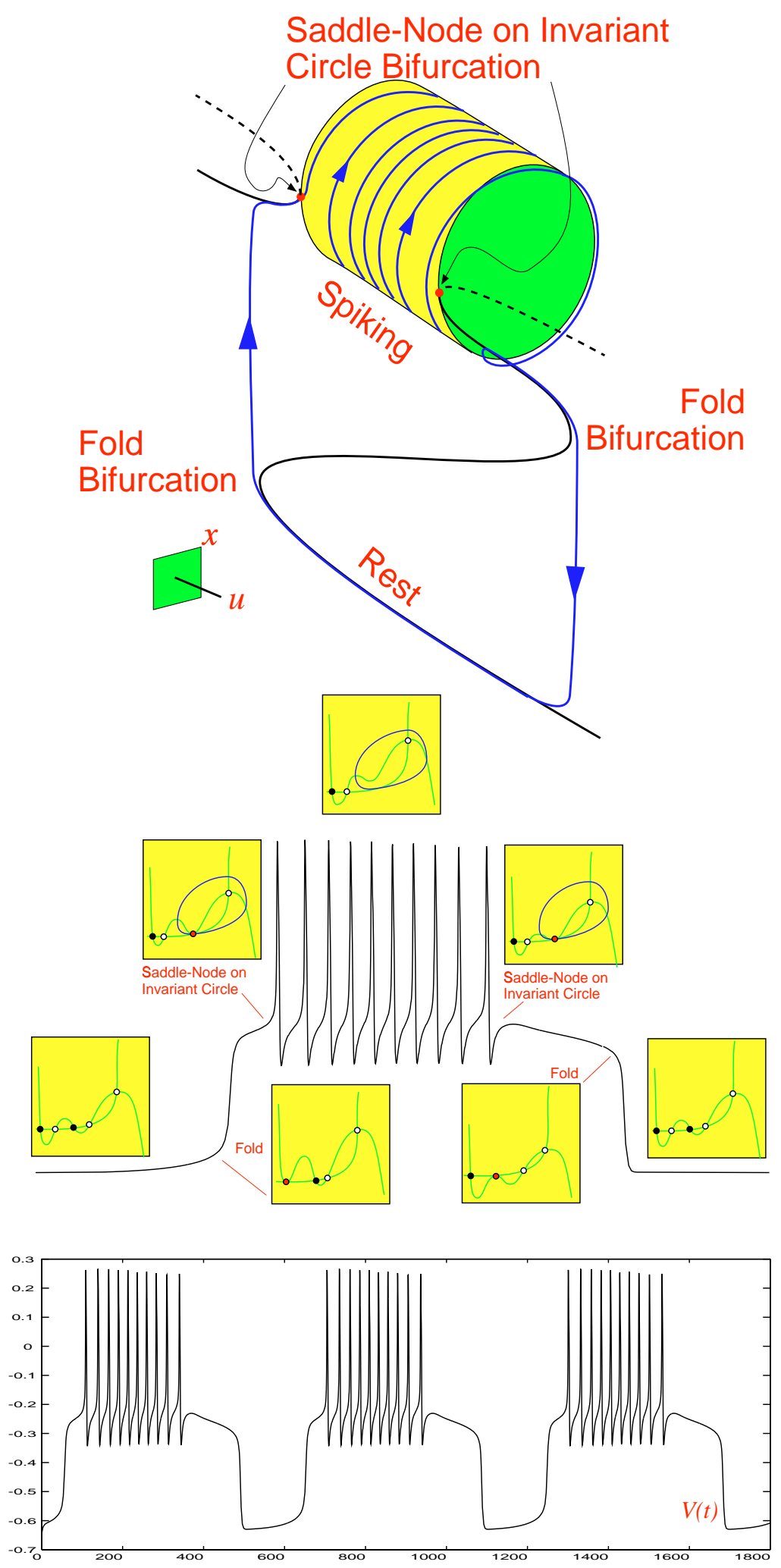

Fig. 52. "Circle/circle" ("parabolic") bursting via "fold/fold" hysteresis loop: The transition from quiescent state to repetitive spiking and back occurs via a saddle-node on invariant circle bifurcation. The transitions between the down-state (lower branch) and the up-state (upper branch) occur via fold bifurcations. Shown are simulations of the Morris-Lecar system with two additional low-threshold currents: Inward current as in Fig. 62 and an outward current $I_{R}(V, u)=0.2(1+\tanh \{(V+$ $0.3) / 0.1\}) u^{4}\left(E_{\mathrm{k}}-V\right)$. The other parameters and variables are as in Fig. 62 except $I=-0.067$ and $\mu=0.0025$. 


\section{Bifurcation of Spiking State}

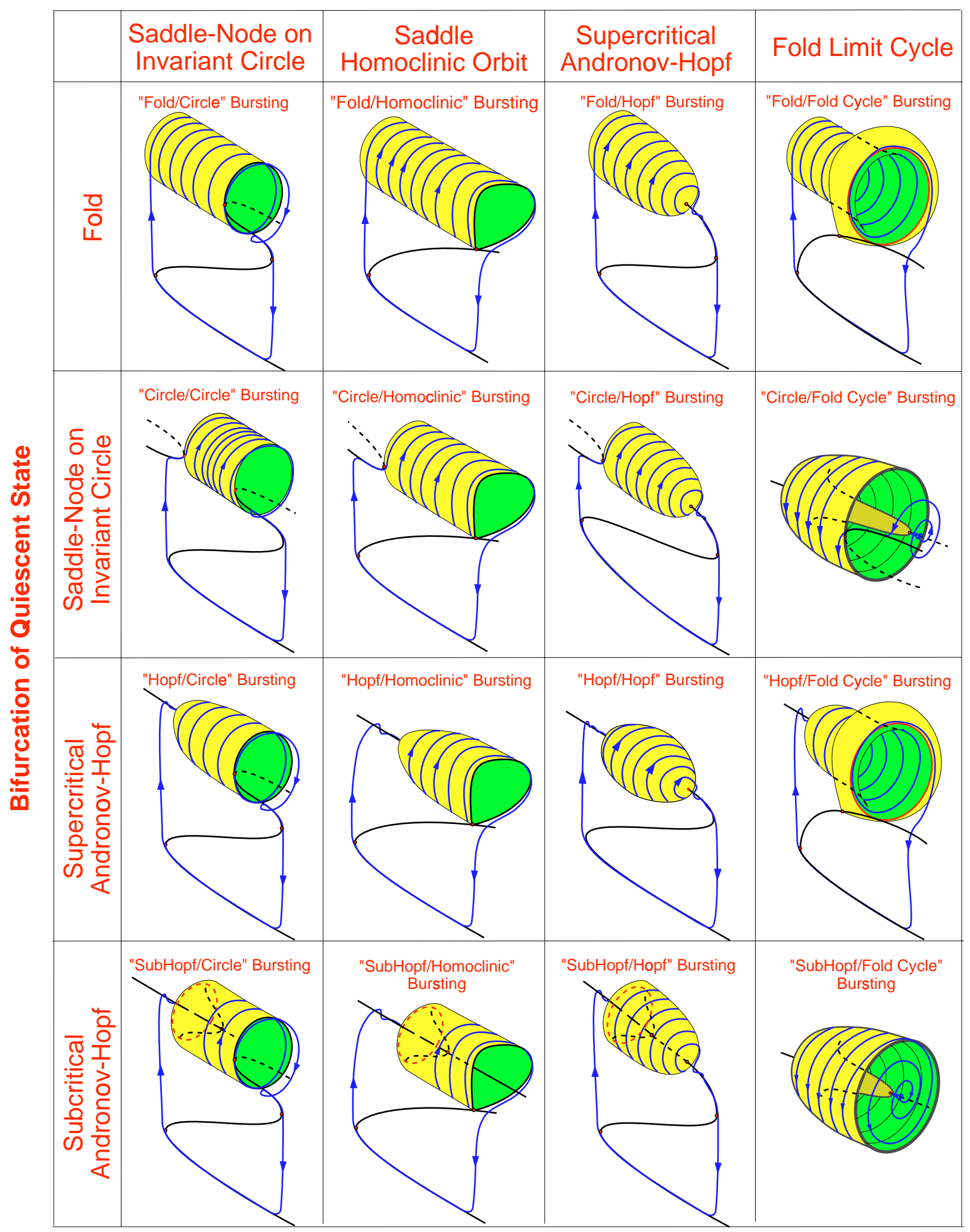

Fig. 53. Examples of planar point-cycle fast-slow codimension 1 bursters of hysteresis loop type. 

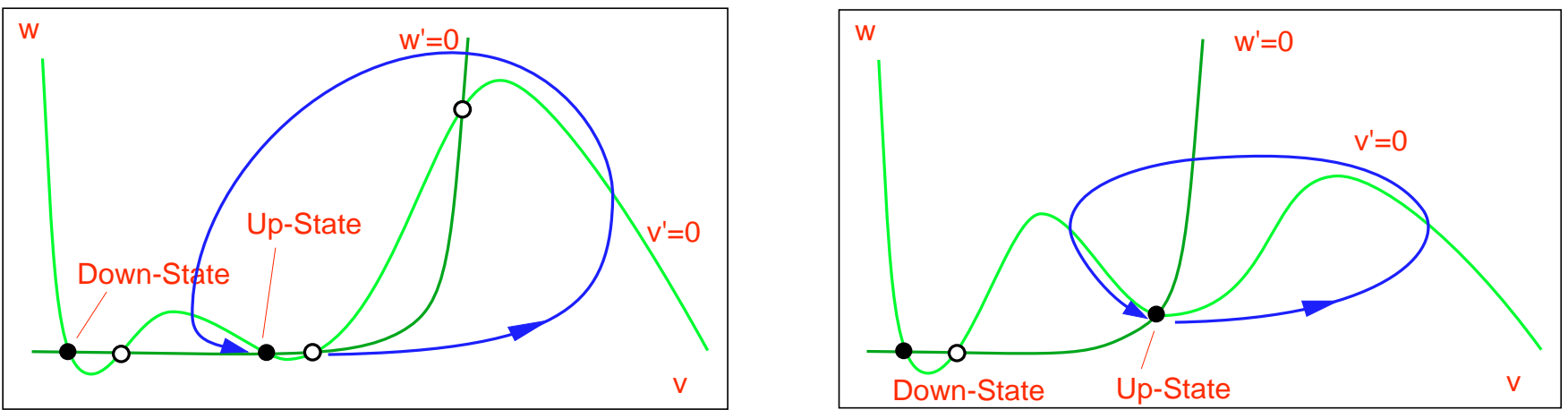

Fig. 54. Possible intersections of nullclines in planar system $v^{\prime}=f(v, w), w^{\prime}=\mu g(v, w)$ that would result in the two-state behavior depicted in Fig. 50 with only the up-state being excitable (notice the blue periodic pseudo-orbits). Here $v$ and $w$ are the voltage and recovery variables, respectively.
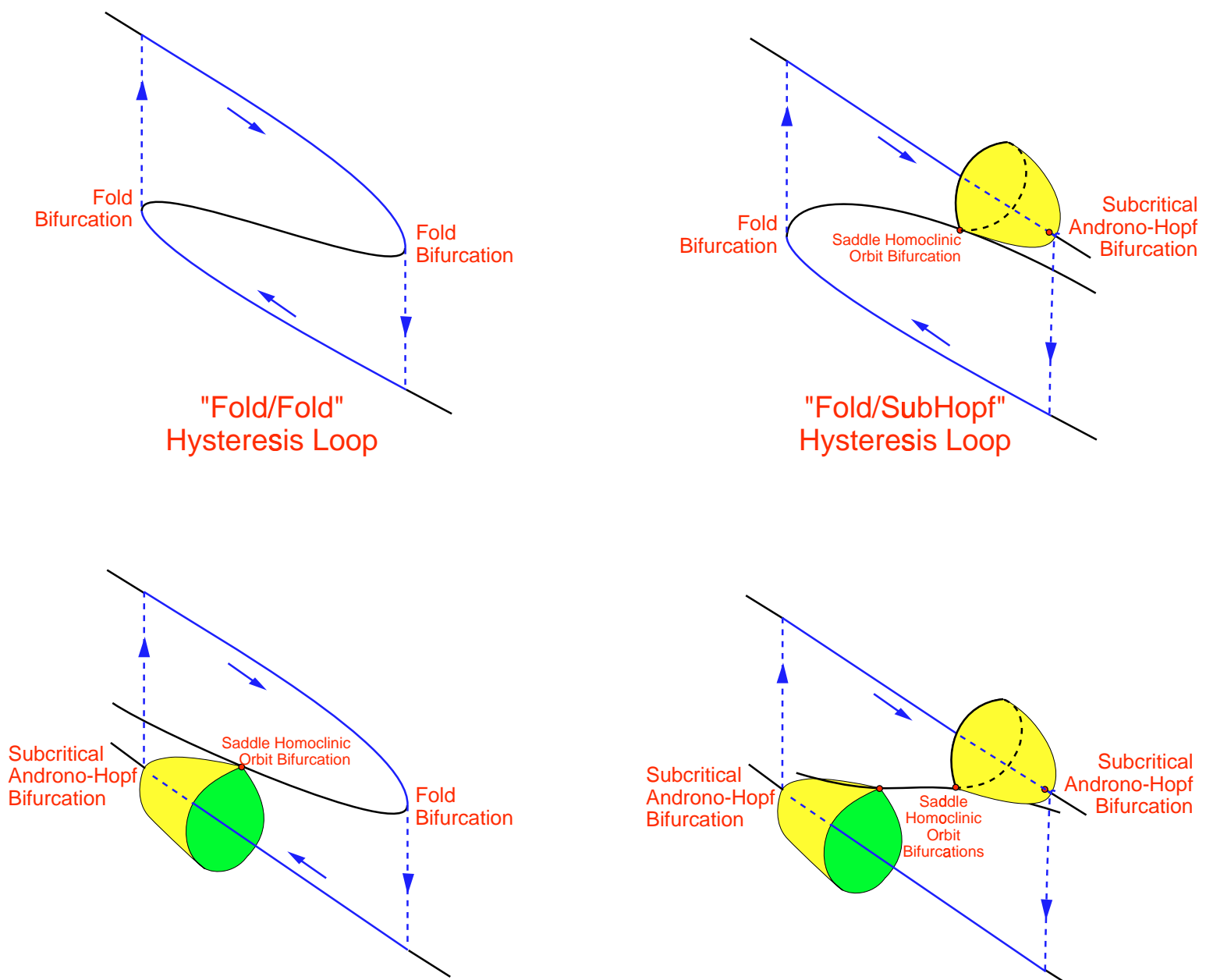

"SubHopf/Fold"

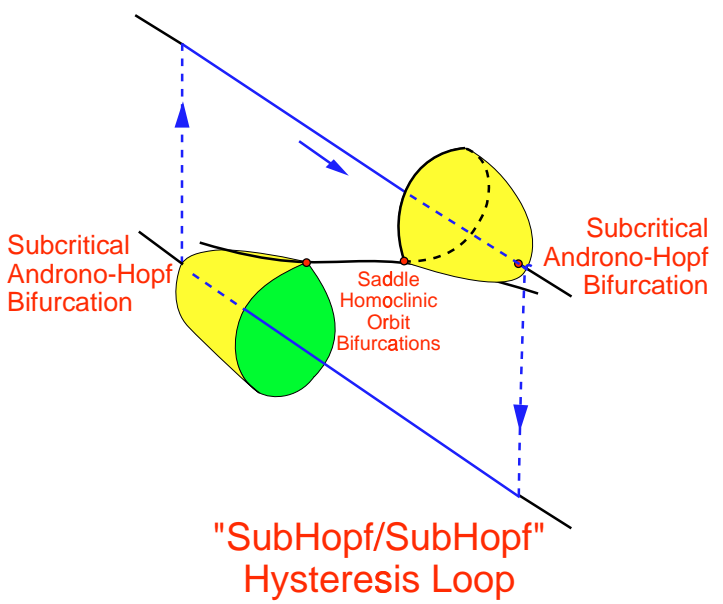

Fig. 55. Classification of point-point hysteresis loops. 


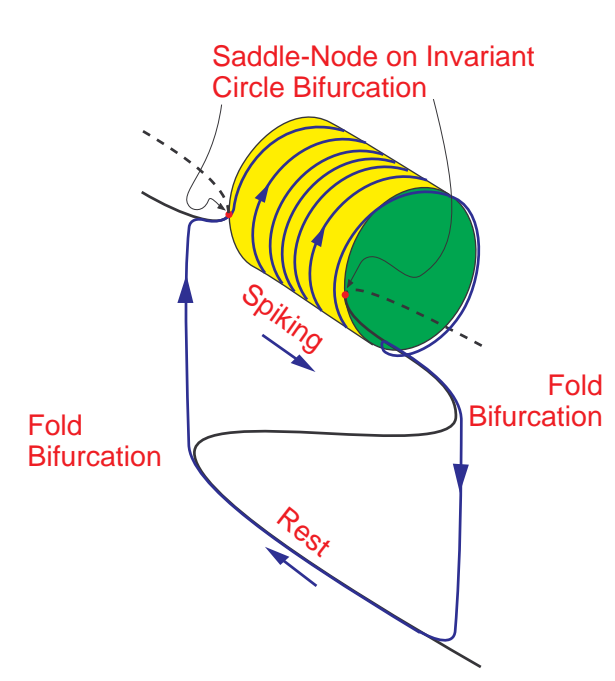

"Circle/Circle" Bursting
via "Fold/Fold"
Hysteresis Loop

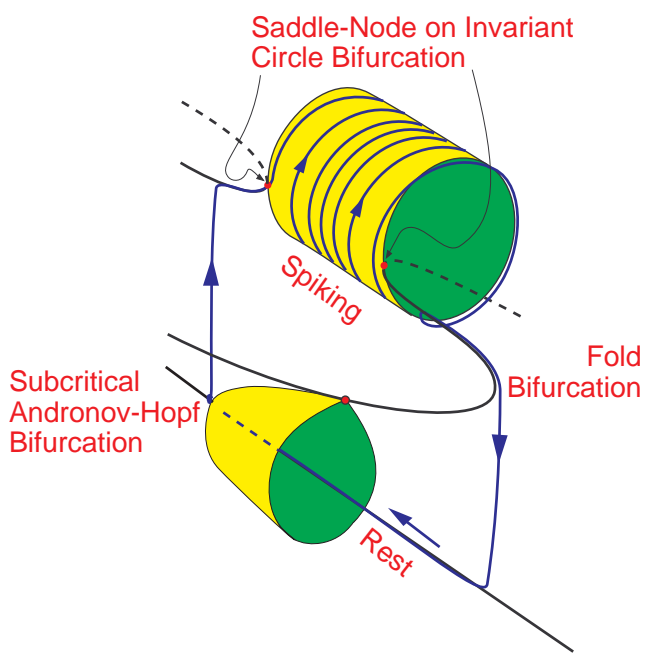

"Circle/Circle" Bursting via "SubHopf/Fold" Hysteresis Loop
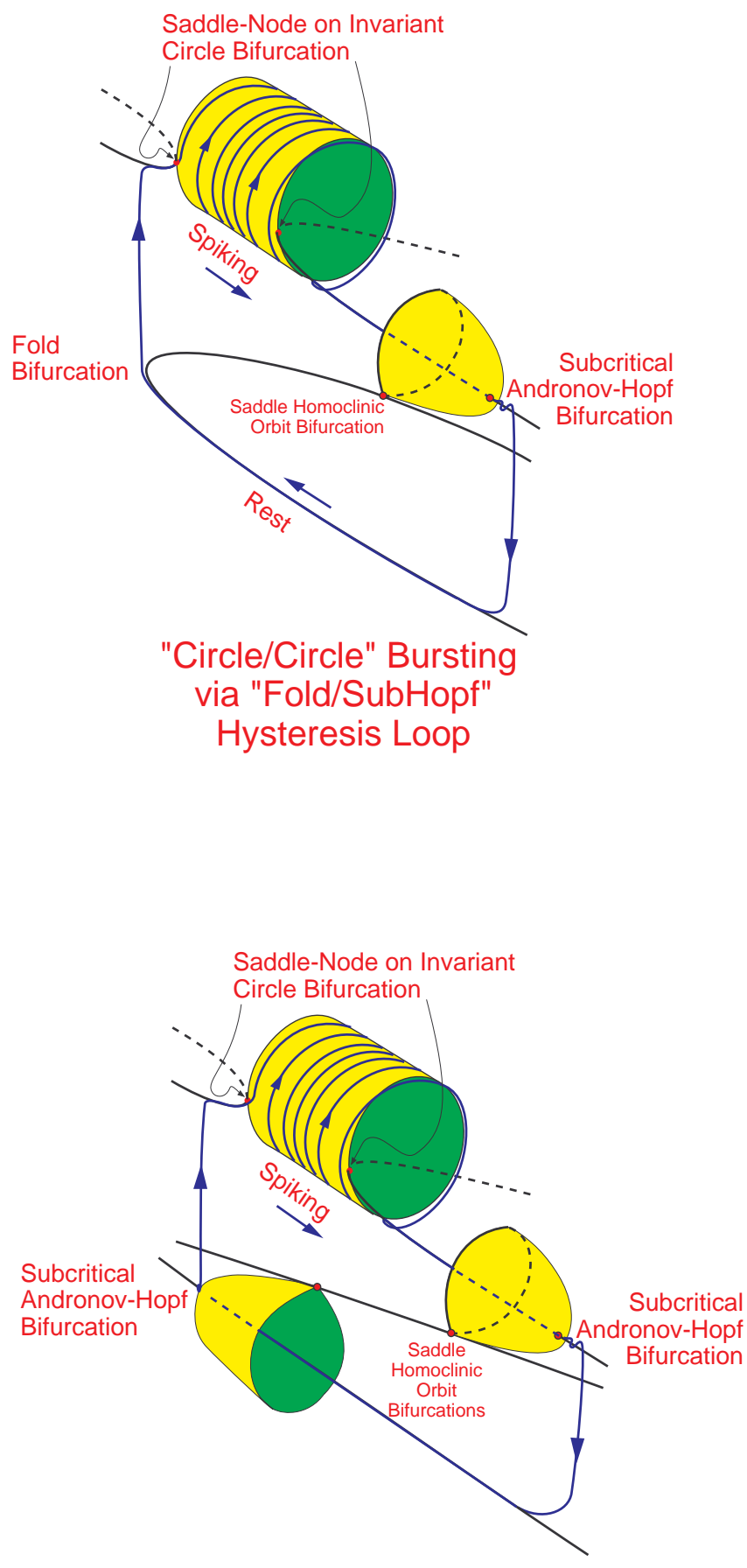

"Circle/Circle" Bursting via "SubHopf/SubHopf" Hysteresis Loop

Fig. 56. Subtypes of the "circle/circle" hysteresis loop bursting. See Fig. 55 for reference. 

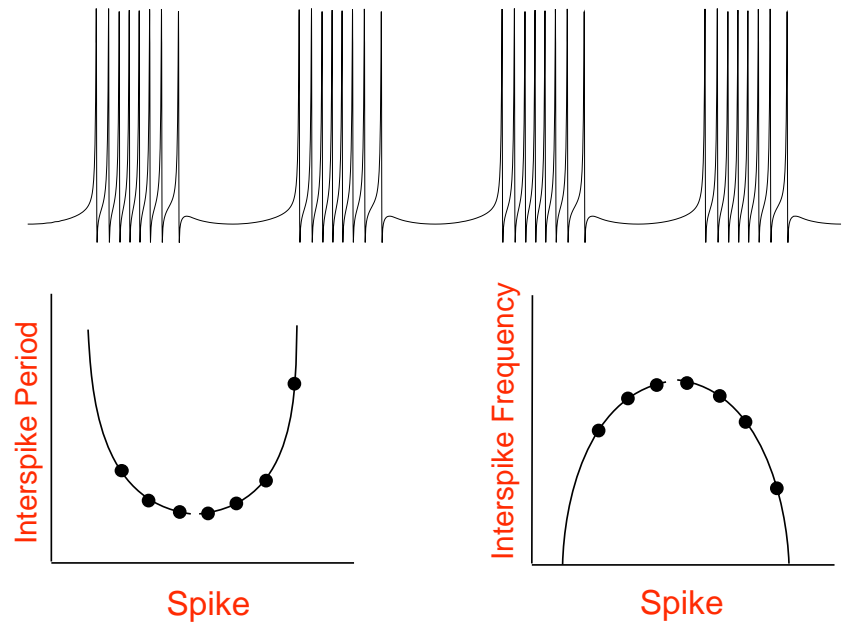

Fig. 57. The interspike period in the "circle/circle" bursting resembles a parabola, which motivates the name "parabolic bursting".

behaves as $\sqrt{\lambda}$, where $\lambda$ is the distance to the bifurcation, the interspike period "looks" like a parabola (see Fig. 57), which motivates the name "parabolic bursting". Such a bursting is exhibited by the $R_{15}$ cells in the abdominal ganglion of the mollusk Aplysia and it has been studied by many scientists, including Plant [1981], Rinzel and Lee [1987], Ermentrout and Kopell [1986a, 1986b], Canavier et al. [1991], Bertram [1993], Baer et al. [1995], Bertram et al. [1995], Soto-Trevino et al. [1996], and Butera et al. [1996, 1997], Hoppensteadt [1997], and Taylor and Holmes [1998].

Under fairly general conditions, any slow wave "circle/circle" burster can be transformed to the Ermentrout-Kopell canonical model [Ermentrout \& Kopell, 1986a, 1986b; Hoppensteadt \& Izhikevich, 1997]

$$
\begin{aligned}
& \dot{\vartheta}=1-\cos \vartheta+(1+\cos \vartheta) r(\psi) \\
& \dot{\psi}=\omega
\end{aligned}
$$

where $r: \mathbb{S}^{1} \rightarrow \mathbb{R}$ is some function that depends on particulars of the burster, e.g. $r(\psi)=\cos \psi$. When $r(\psi)<0$, the cell is quiescent; When $r(\psi)>0$, it fires periodically; see Fig. 58. As was shown by Soto-Trevino et al. [1995], the slow subsystem does not need a limit cycle attractor to exhibit periodic bursting; it might be just excitable; see detailed discussion in Sec. 8.2.3 by Hoppensteadt and Izhikevich [1997].

As we discussed above, there can be at least four subtypes of hysteresis loop "circle/circle" bursting having one-dimensional slow variable (i.e. " $2+1$ " bursting); see Figs. 52 and 56 .

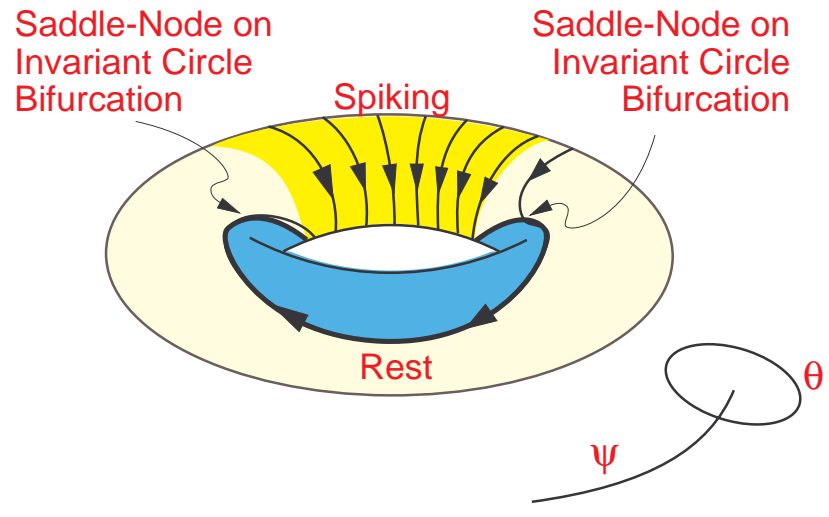

Fig. 58. "Circle/circle" ("parabolic") slow wave bursting. Variables $\theta, \psi \in \mathbb{S}^{1}$.

\subsection{5. "Fold/homoclinic" ("square-wave") bursting}

The rest state disappears via fold bifurcation, and the periodic spiking disappears via saddlehomoclinic orbit bifurcation; see Fig. 47. Since there is a coexistence of attractors, such bursting occurs via "fold/homoclinic" hysteresis loop; see Fig. 59. It is often referred to as being "squarewave" bursting due to the voltage amplitude profile; see however Fig. 123.

This type of bursting is exhibited by pancreatic $\beta$-cells; see [Chay \& Keizer, 1983; Pernarowski et al., 1992] and references therein. It is ubiquitous in many systems [Alexander \& Cai, 1991] including Hindmarsh and Rose [1984] model [Wang, 1993], Chay-Cook [Bertram et al., 1995] model, and the reduced Morris-Lecar model

$$
\begin{aligned}
\dot{V}= & I-g_{l}\left(V-E_{l}\right)-g_{\mathrm{k}} w\left(V-E_{\mathrm{k}}\right) \\
& -g_{\mathrm{Ca}} m_{\infty}(V)\left(V-E_{\mathrm{Ca}}\right) \\
\dot{w}= & \lambda(V)\left(w_{\infty}(V)-w\right)
\end{aligned}
$$

where

$$
\begin{aligned}
m_{\infty}(V) & =\frac{1}{2}\left(1+\tanh \frac{V-V_{1}}{V_{2}}\right) \\
w_{\infty}(V) & =\frac{1}{2}\left(1+\tanh \frac{V-V_{3}}{V_{4}}\right) \\
\lambda(V) & =\frac{1}{3} \cosh \frac{V-V_{3}}{2 V_{4}} .
\end{aligned}
$$

We assume $I(u)=-u$ and use a linear slow subsystem

$$
\dot{u}=\mu(0.2+V)
$$



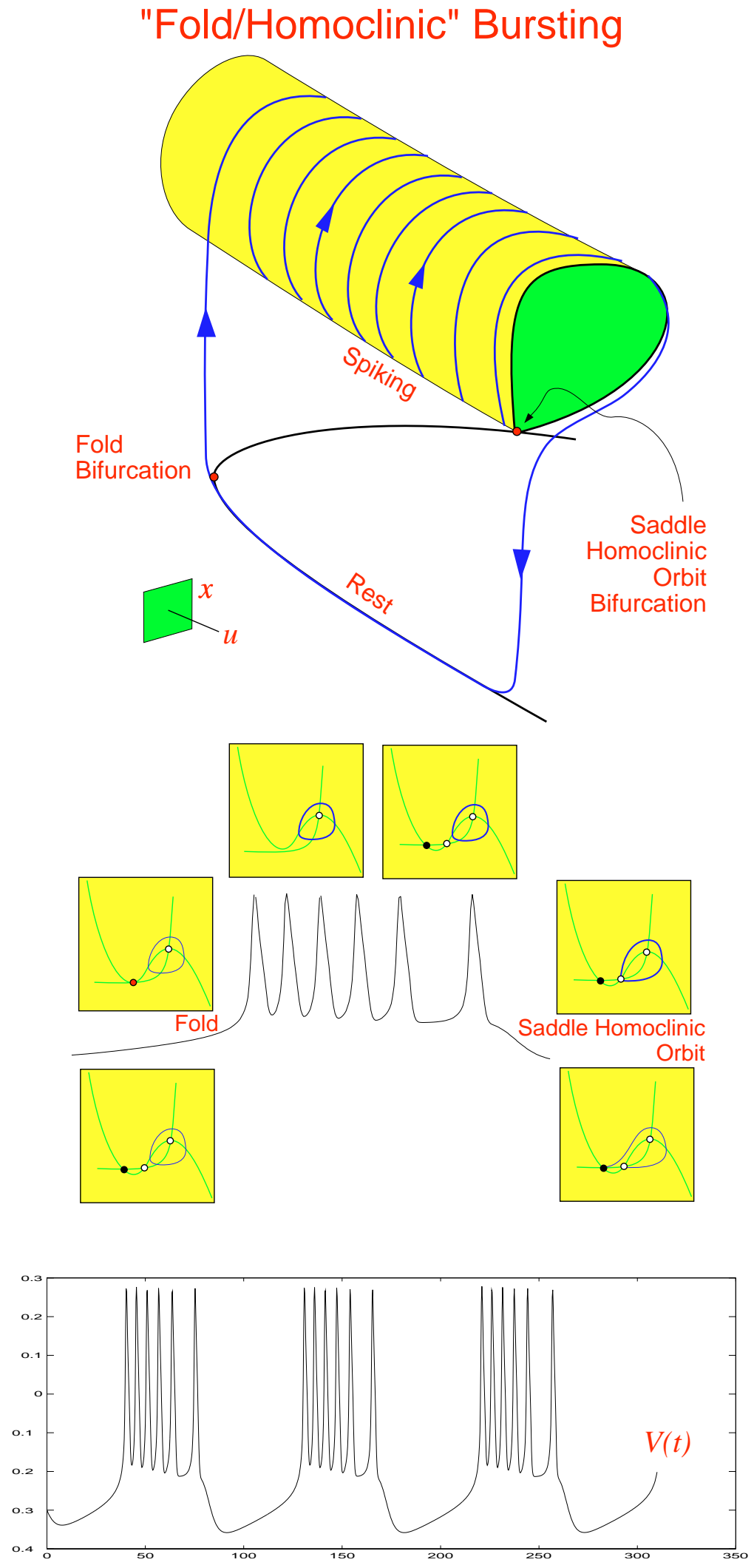

Fig. 59. "Fold/homoclinic" ("square-wave") bursting: The rest state disappears via fold bifurcation and the limit cycle disappears via saddle homoclinic orbit bifurcation. The same bifurcations form the "fold/homoclinic" hysteresis loop. (Modified from [Hoppensteadt \& Izhikevich, 1997].) Simulations of the Morris-Lecar system (25) with slow equation (26). Parameters: $\left(V_{1}, V_{2}, V_{3}, V_{4}, E_{l}, E_{\mathrm{k}}, E_{\mathrm{Ca}}, g_{l}, g_{\mathrm{k}}, \mathrm{g}_{\mathrm{Ca}}\right)=(-0.01,0.15,0.1,0.05,-0.5,-0.7,1,0.5,2,1.2)$ and $\mu=0.005$. 
to obtain "fold/homoclinic" bursting depicted in Fig. 59, as well as many other types. Mathematical aspects of "fold/homoclinic" bursting have been studied by Terman [1991] who showed that the burster could have a Smale horseshoe structure leading to chaotic dynamics.

When the two bifurcations occur for nearby values of the slow variable, the fast subsystem is near saddle-node separatrix loop bifurcation; see Sec. 2.3. Under fairly general conditions on the dynamics of the slow subsystem, the burster can be transformed into the canonical model

$$
\begin{aligned}
& \dot{\vartheta}=1-\cos \vartheta+(1+\cos \vartheta) u \\
& \dot{u}=\mu
\end{aligned}
$$

where each spike resets $\vartheta$ to $a$ and decreases $u$ by a constant $b$; i.e.

$$
\vartheta(t) \leftarrow a \text { and } u(t) \leftarrow u(t)-b
$$

as soon as $\vartheta(t)=\pi$. The canonical model exhibits "fold/homoclinic" hysteresis loop periodic bursting for any $0<a<\pi$, any $b>0$ and sufficiently small $\mu$; see Fig. 60 .

\subsection{6. "Fold/circle" ("triangular") bursting}

The quiescent state disappears via fold bifurcation, and the periodic limit cycle attractor corresponding to repetitive spiking disappears via saddle-node on invariant circle bifurcation; see Figs. 47 and 61 . Such bursting has been studied by Rush and Rinzel [1994] in a thalamic neuron model, and it is referred to as being "triangular" bursting [Wang \& Rinzel, 1995].
The bursting can occur via "fold/fold" hysteresis loop, as we depict in Fig. 61, or via "fold/subHopf" hysteresis loop (not shown).

\subsection{7. "Circle/homoclinic" bursting}

The quiescent state disappears via saddle-node on invariant circle bifurcation, and the periodic limit cycle attractor corresponding to repetitive spiking disappears via saddle homoclinic orbit bifurcation; see Figs. 47 and 62. Such a bursting can also occur via "subHopf/homoclinic" hysteresis loop (not shown).

\subsection{8. "Fold/Hopf" ("tapered") bursting}

The rest state disappears via fold bifurcation, and the periodic spiking disappears via supercritical Andronov-Hopf bifurcation; see Fig. 63. Such bursting, also known as "tapered", was studied by Holden and Erneux [1993a, 1993b] in the context of slow passage through the supercritical Hopf bifurcation. It is exhibited by pancreatic $\beta$-cell models [Smolen et al., 1993; Pernarowski, 1994; de Vries, 1998] and by coupled Hindmarsh-Rose neurons [Abarbanel et al., 1996]. It can occur via "fold/fold" hysteresis loop, as depicted in Fig. 63, or via any other point-point hysteresis loop from Fig. 55.

\subsection{9. "Circle/fold cycle" bursting}

The rest state disappears via saddle-node on invariant circle bifurcation, and the periodic spiking disappears via fold limit cycle bifurcation. It can occur

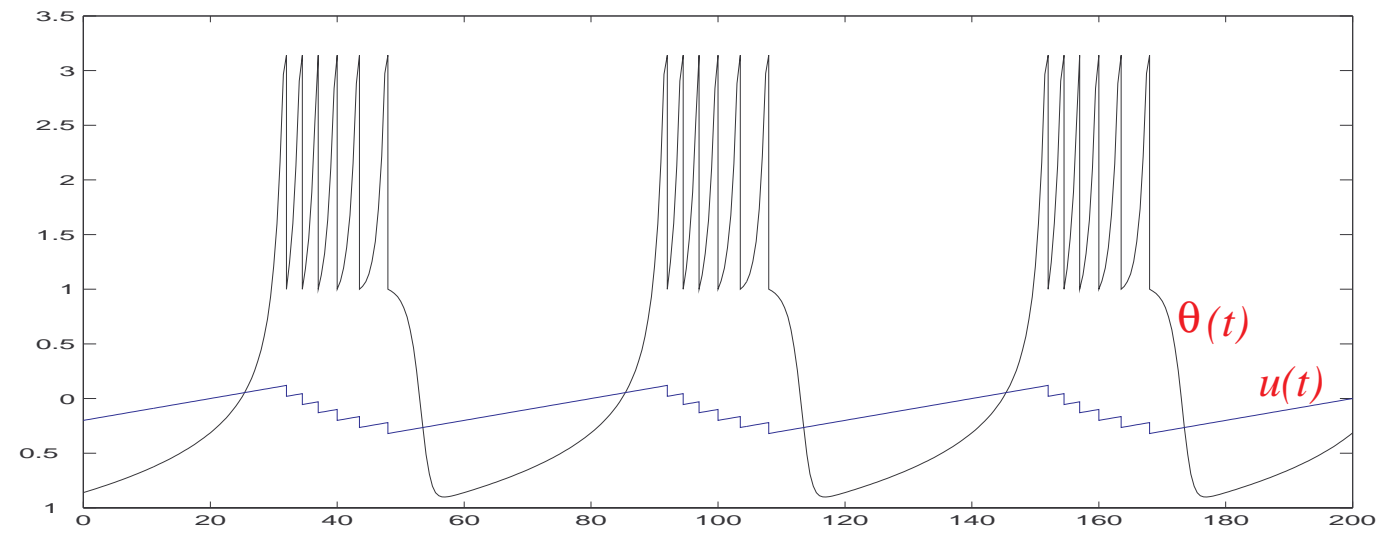

Fig. 60. Typical bursting solution of (27). Parameters: $a=1, b=0.1$, and $\mu=0.01$. 


\section{"Fold/Circle" Bursting}
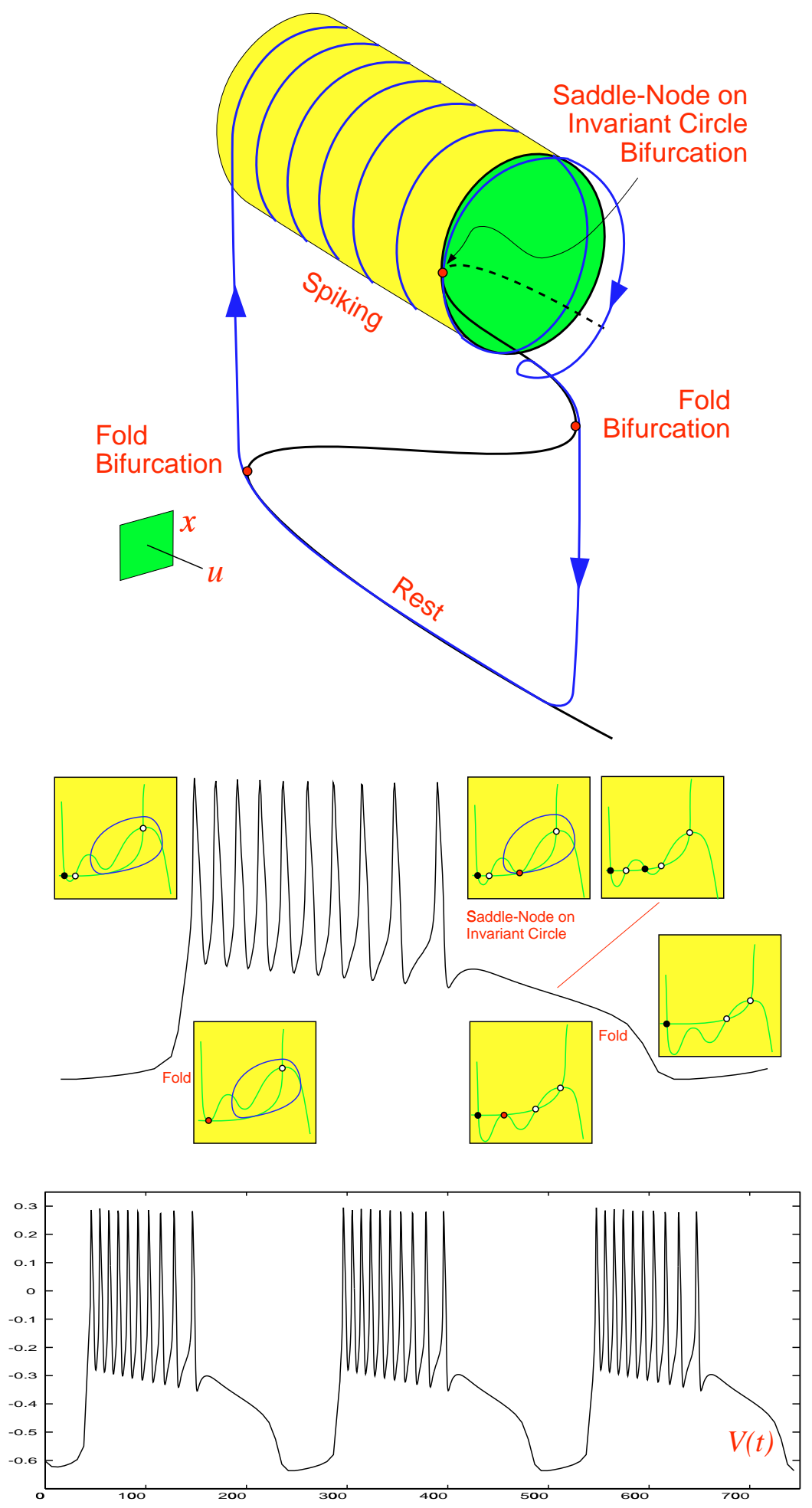

Fig. 61. "Fold/circle" ("triangular") bursting via "fold/fold" hysteresis loop: The rest state disappears via fold bifurcation, and the repetitive spiking disappears via saddle-node on invariant circle bifurcation. Simulations of the Morris-Lecar system (25) with parameters as in Fig. 59 and an additional slowly inactivating low-threshold current $I_{\mathrm{T}}(V, u)=0.1|u|(1+\tanh \{(V-$ $\left.\left.\left.V_{5}\right) / 0.1\right\}\right)\left(E_{\mathrm{Ca}}-V\right)$ where $V_{5}=-0.5, u^{\prime}=\mu \lambda(V)(-0.5-V)$ and $\mu=0.01$. 


\section{"Circle/Homoclinic" Bursting}
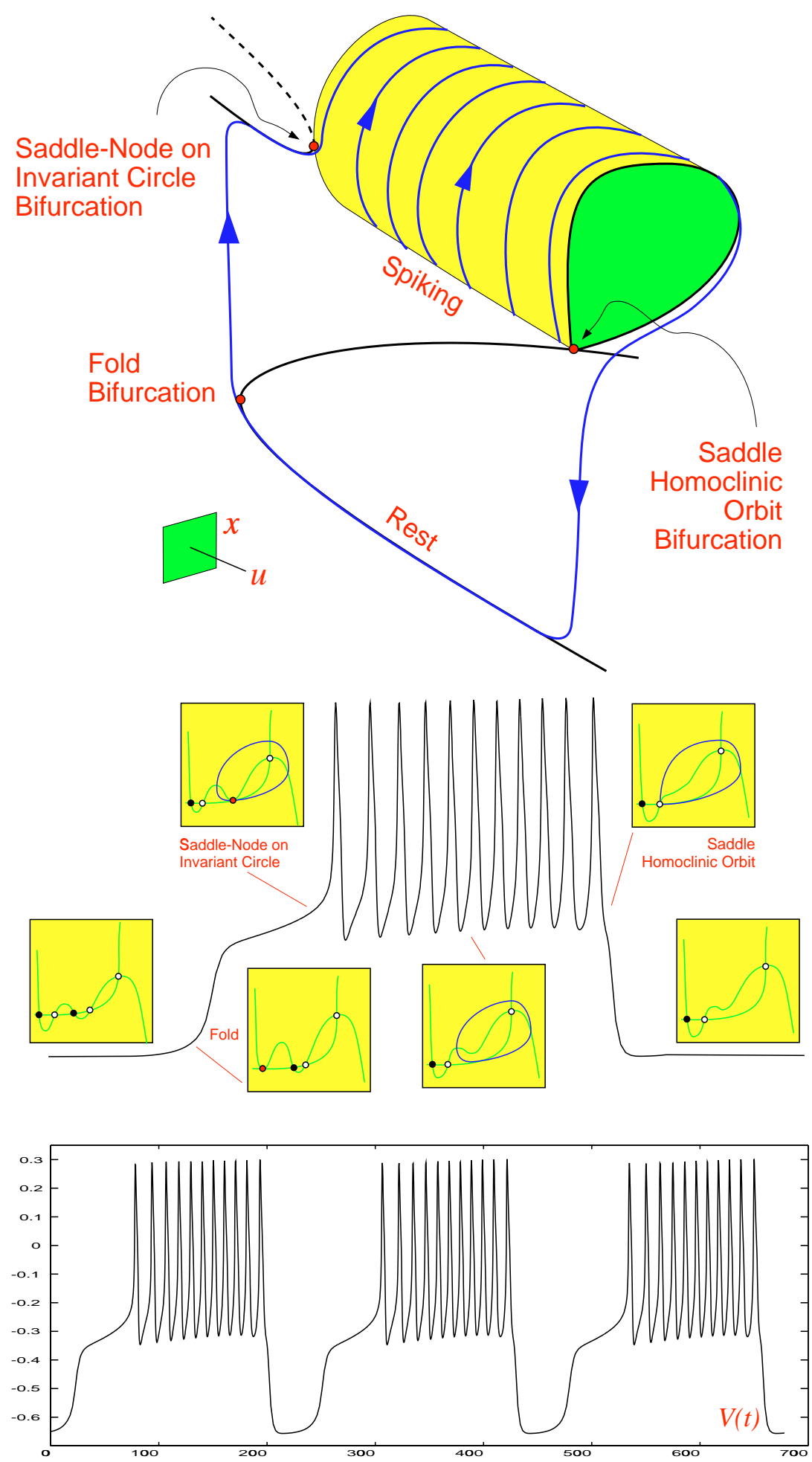

Fig. 62. "Circle/homoclinic" bursting via "fold/homoclinic" hysteresis loop: The rest state disappears via saddle-node on invariant circle bifurcation, and the spiking state disappears via saddle homoclinic orbit bifurcation. Simulations of the Morris-Lecar system with additional current as in Fig. 61 except that $V_{5}(u)=-0.8-0.5 u$. 


\section{"Fold/Hopf" Bursting}
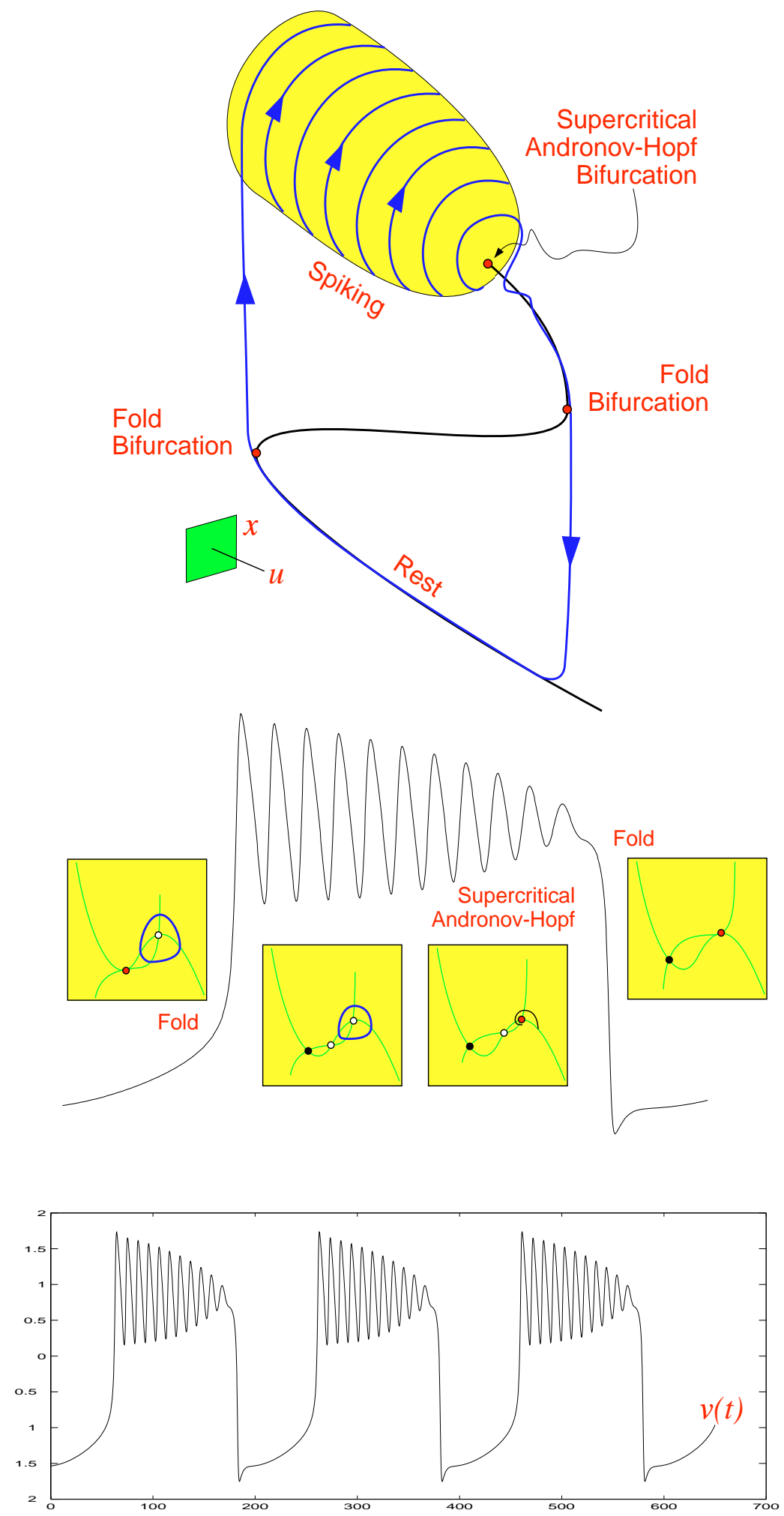

Fig. 63. "Fold/Hopf" ("tapered") bursting via "fold/fold" hysteresis loop: The rest state disappears via fold bifurcation, and the repetitive spiking disappears via supercritical Andronov-Hopf bifurcation. Simulations of (15) with slow subsystem $\dot{c}=\mu v$. Parameters: $(a, b, d, \varepsilon, \mu)=(-1.55,2.5,0.1,0.5,0.01)$. 


\section{"Circle/Fold Cycle" Bursting}

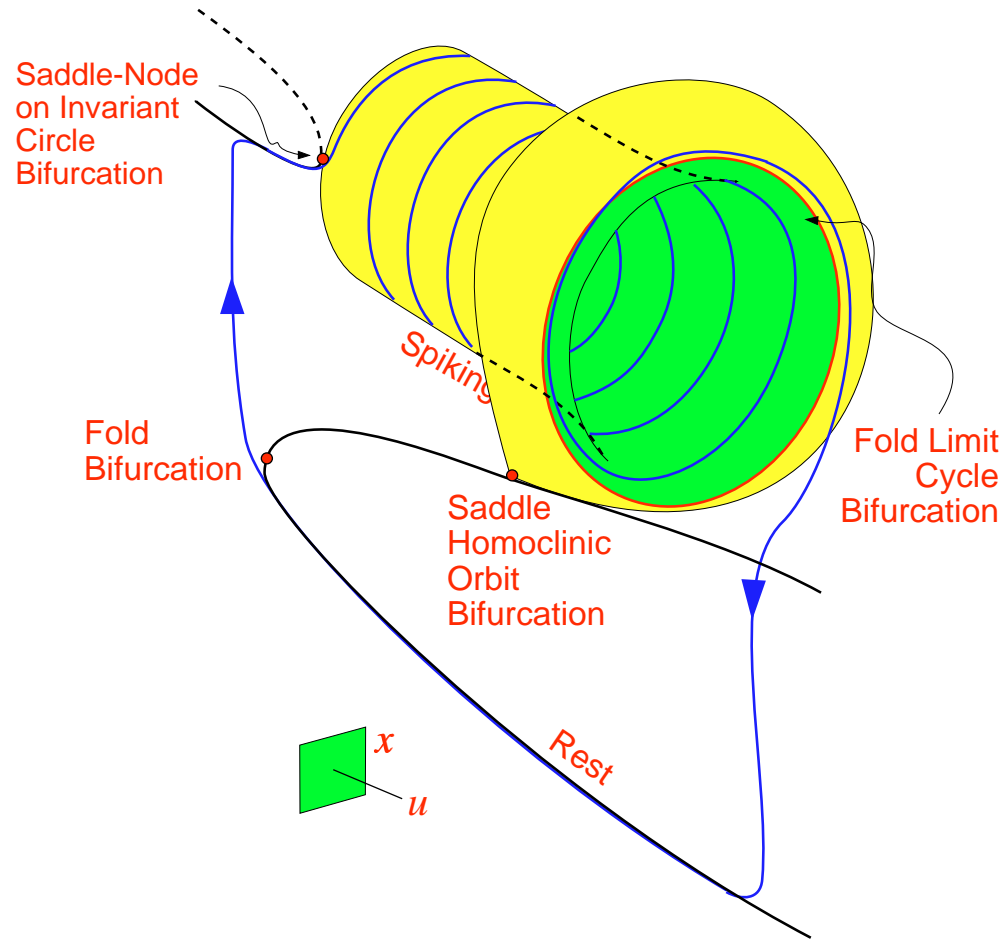

Fig. 64. "Circle/fold cycle" bursting via "fold/fold cycle" hysteresis loop: The rest state disappears via saddle-node on invariant circle bifurcation, and the repetitive spiking disappears via fold limit cycle bifurcation. See also Figs. 65 and 66 .
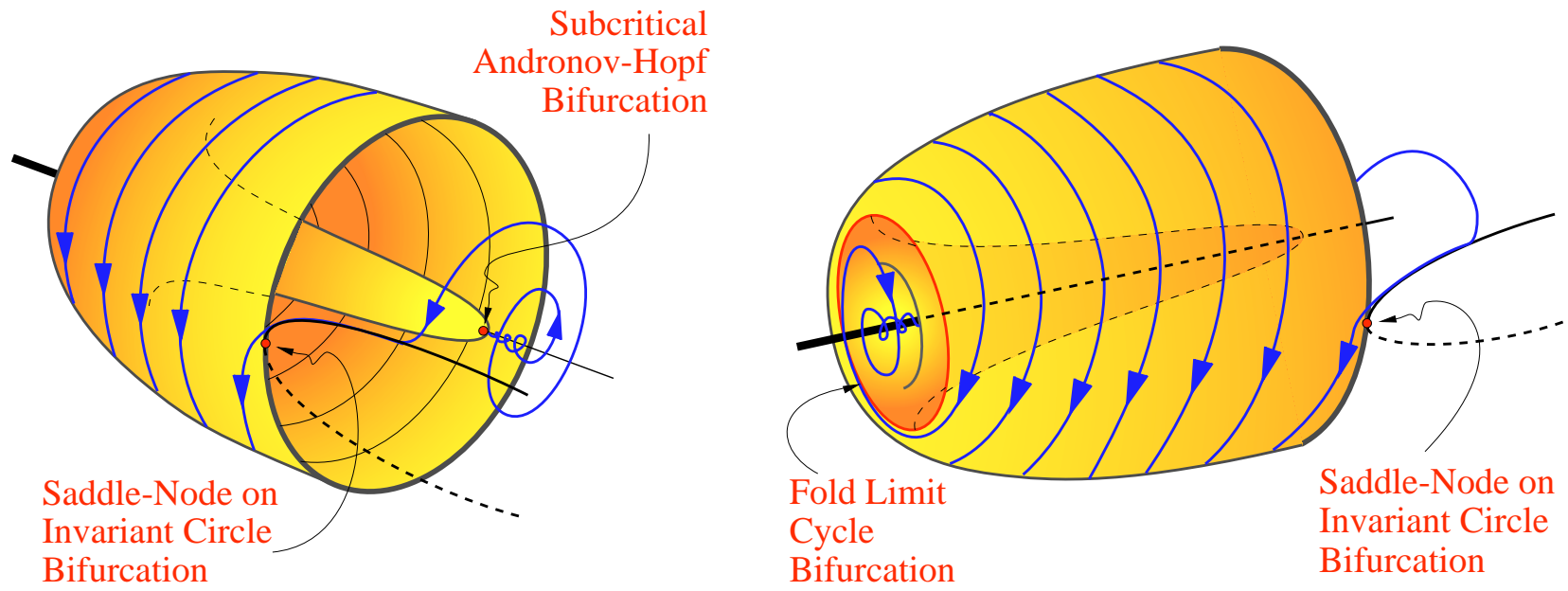

Fig. 65. "Circle/fold cycle" bursting via "subHopf/fold cycle" hysteresis loop: The rest state disappears via saddle-node on invariant circle bifurcation, and the repetitive spiking disappears via fold limit cycle bifurcation. 
via "fold/fold cycle hysteresis loop, as depicted in Fig. 64, or via "subHopf/fold cycle" hysteresis loop as depicted in Figs. 65 and 66.

\subsubsection{0. "Circle/Hopf" bursting}

The quiescent state disappears via saddle-node bifurcation on invariant circle, and the periodic limit cycle attractor corresponding to repetitive spiking shrinks to a point via supercritical Andronov-Hopf bifurcation. It can occur via "fold/fold" hysteresis loop as we depict in Fig. 67, or via any other point-point hysteresis loop from Fig. 55.

\subsubsection{1. "Fold/Fold cycle" bursting}

The quiescent state disappears via fold bifurcation, and the periodic limit cycle attractor corresponding to repetitive spiking disappears via fold limit cycle bifurcation; see Fig. 68. Such a bursting was first discovered in Chay-Cook model by Bertram et al. [1995], who referred to it as being a Type IV bursting.

\subsubsection{2. "SubHopf/homoclinic" bursting}

The quiescent state disappears via subcritical Andronov-Hopf bifurcation, and the periodic limit cycle attractor corresponding to repetitive spiking disappears via saddle homoclinic orbit bifurcation. The bursting can occur via "fold/ homoclinic" hysteresis loop, see Fig. 69, or via "subHopf/homoclinic" hysteresis loop as depicted in Fig. 70.

\subsubsection{3. "Hopf/homoclinic" bursting}

The quiescent state disappears via supercritical Andronov-Hopf bifurcation, and the periodic limit cycle attractor corresponding to repetitive spiking disappears via saddle homoclinic orbit bifurcation. The bursting can occur via "fold/homoclinic"
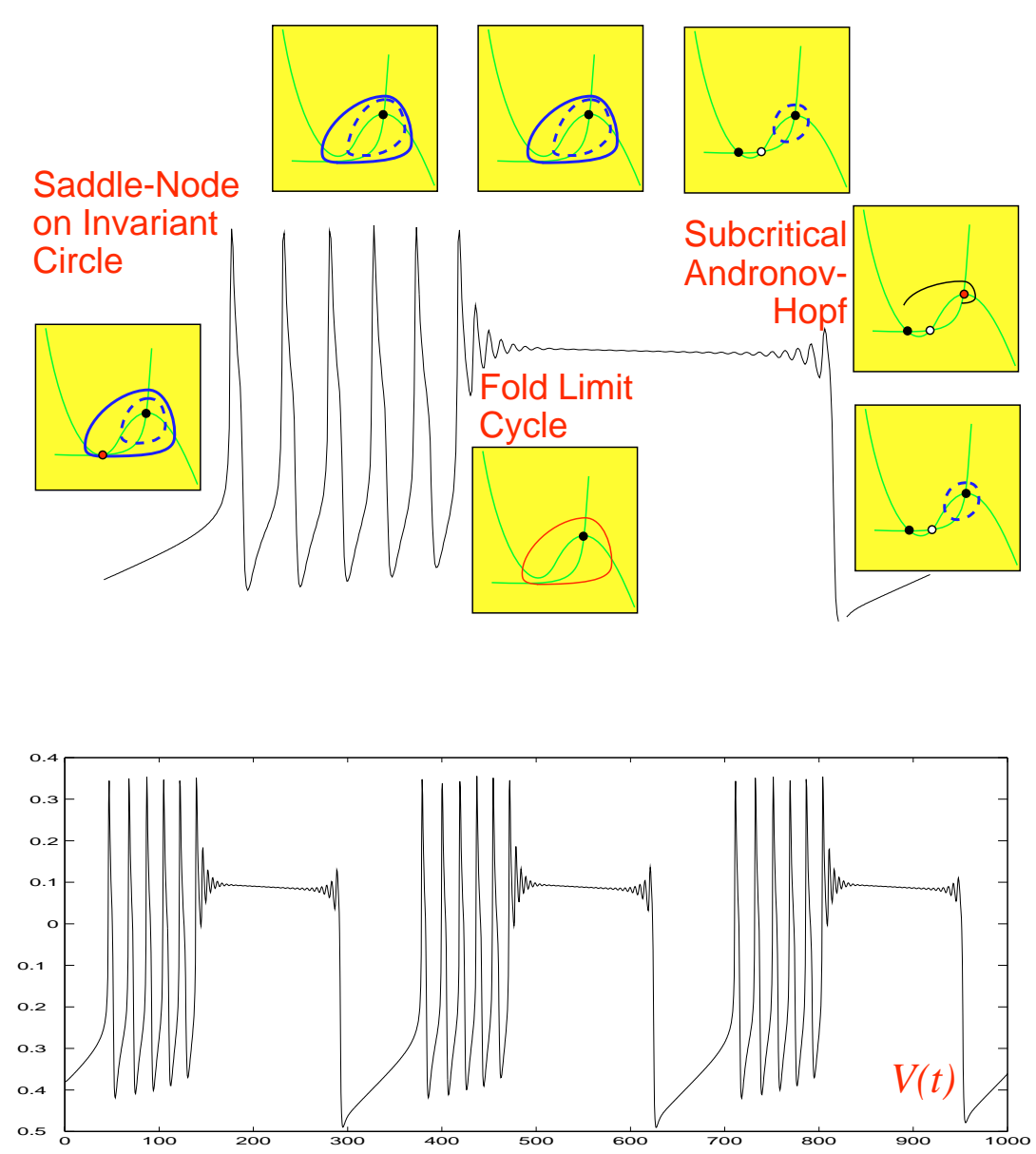

Fig. 66. "Circle/fold cycle" bursting via "subHopf/fold cycle" hysteresis loop (see Fig. 65) in the Morris-Lecar system (25) with slow subsystem $\dot{u}=\mu(0.1+V)$. Parameters $g_{\mathrm{Ca}}=1.36, V_{4}=0.16, \mu=0.003$, the rest as in Fig. 59 . 


\section{"Circle/Hopf" Bursting}
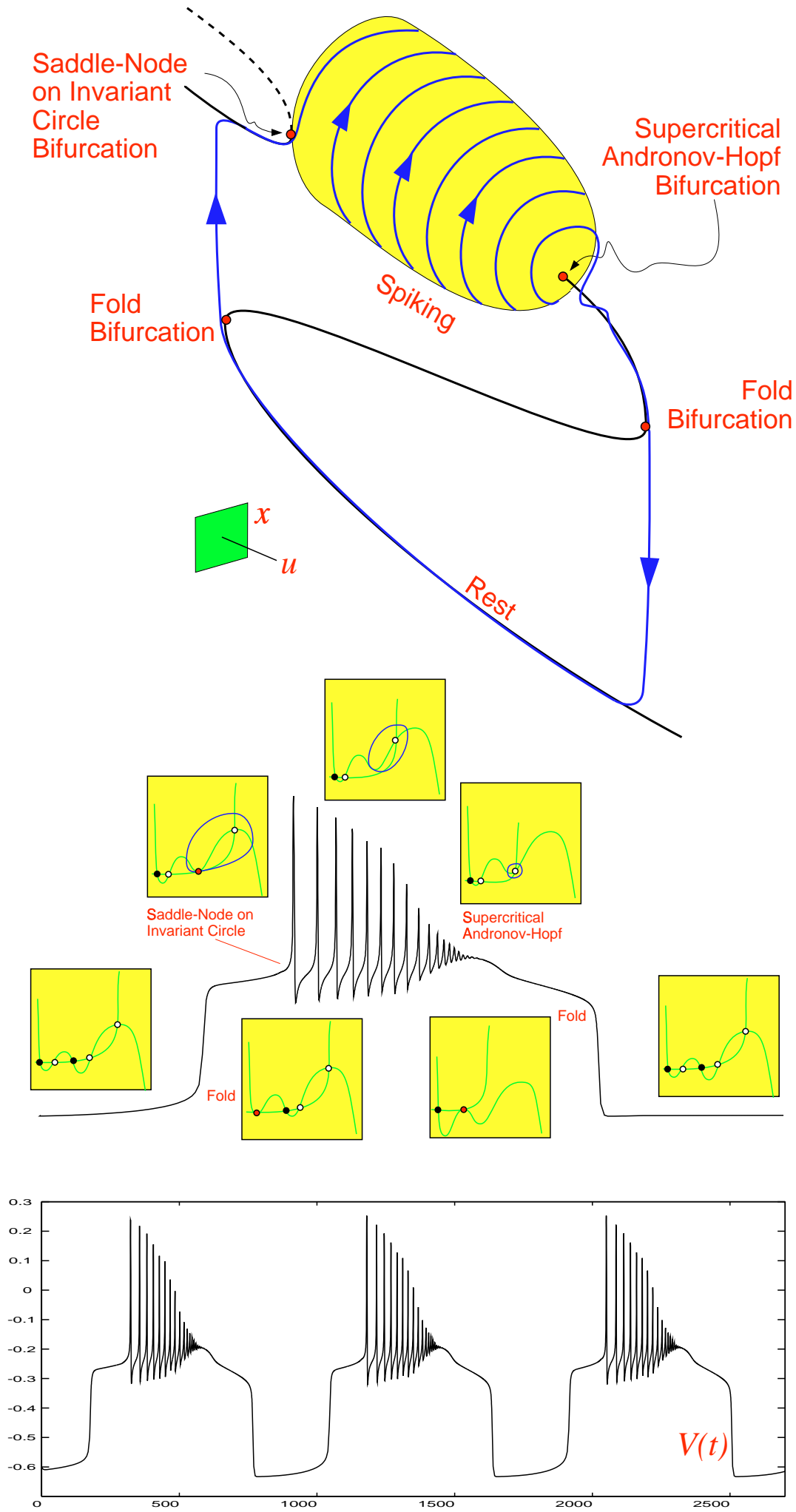

Fig. 67. "Circle/Hopf" bursting via "fold/fold" hysteresis loop: The quiescent state disappears via saddle-node bifurcation on invariant circle, and the periodic spiking disappears via supercritical Andronov-Hopf bifurcation. 


\section{"Fold/Fold Cycle" Bursting}
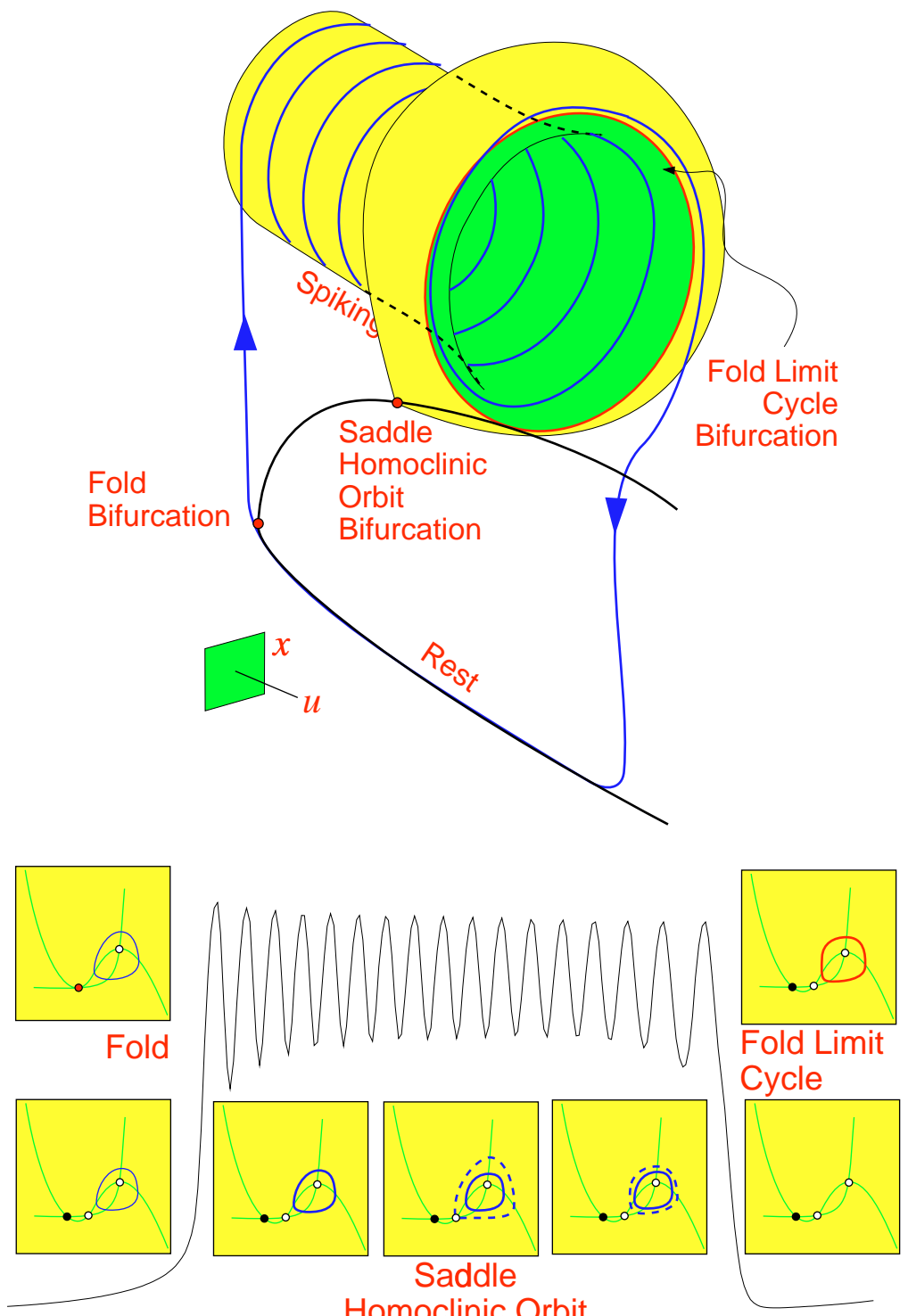

Homoclinic Orbit

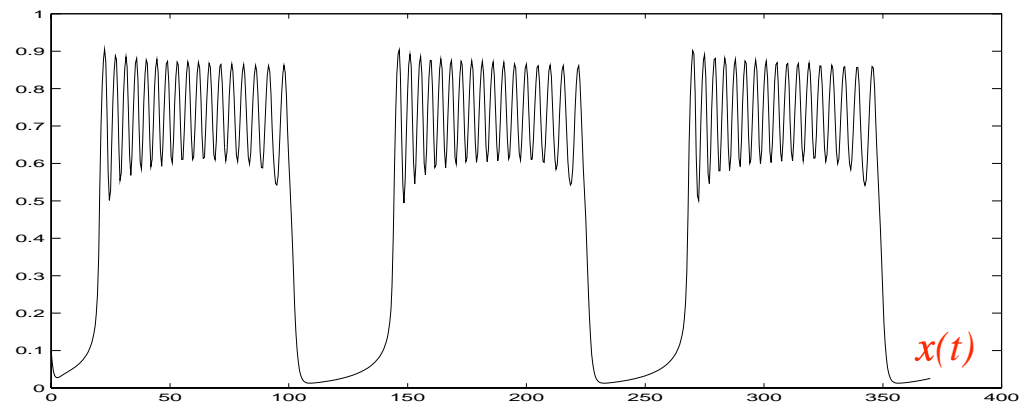

Fig. 68. "Fold/fold cycle" (Type IV) bursting: The quiescent state disappears via fold bifurcation, and the periodic spiking disappears via fold limit cycle bifurcation. The same bifurcations form the "fold/fold cycle" hysteresis loop. Shown are simulations of the Wilson-Cowan [1972] model with parameters as in [Hoppensteadt \& Izhikevich, 1997, Fig. 2.12], and $r_{\mathrm{x}}(u)=-4.76+u, r_{\mathrm{y}}=-9.7+0.3 u$, where $\dot{u}=\mu(0.5-x)$ is a slow subsystem, $\mu=0.1$. 


\section{"SubHopf/Homoclinic" Bursting}
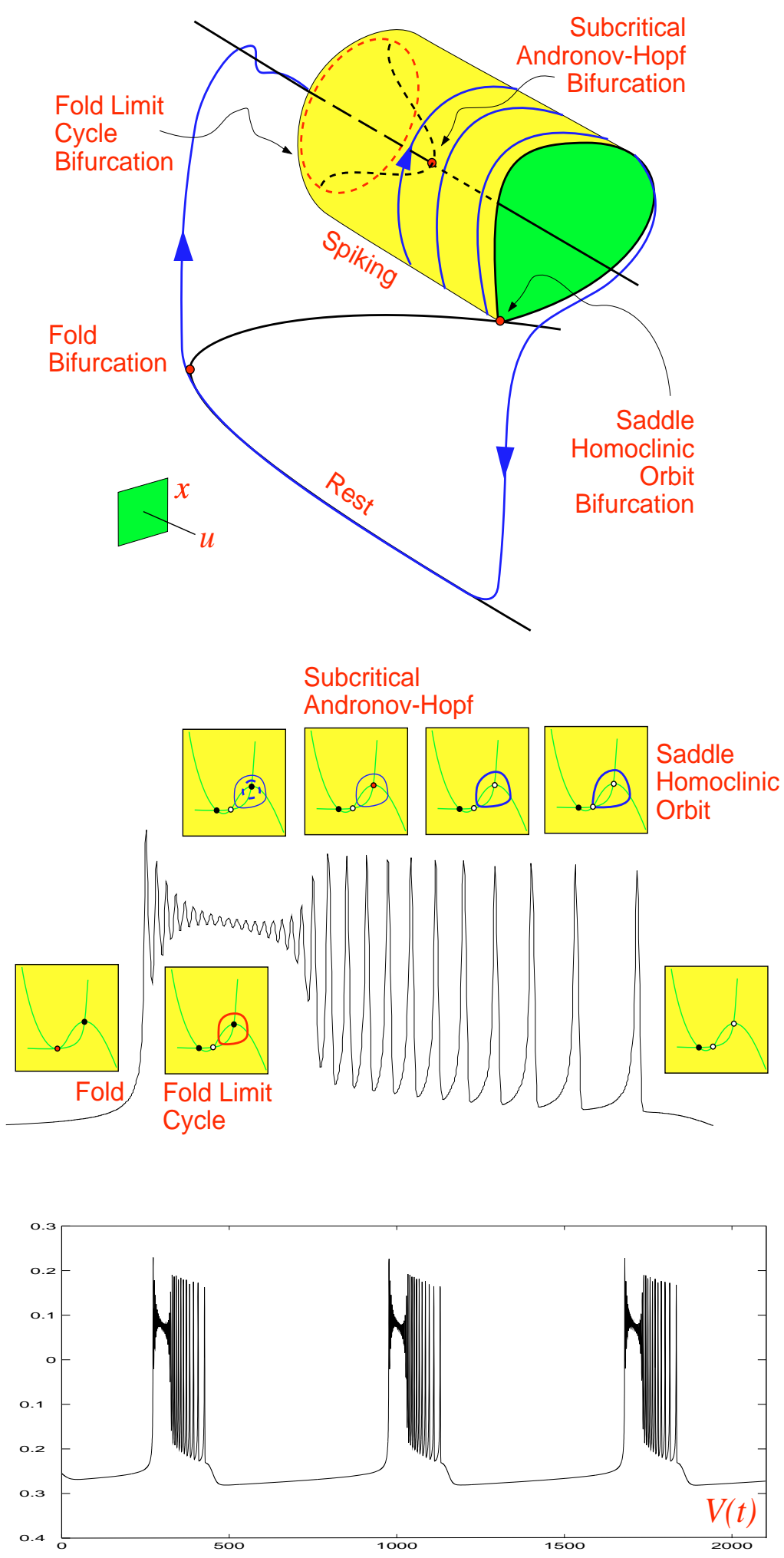

Fig. 69. "SubHopf/homoclinic" bursting via "fold/homoclinic" hysteresis loop: The quiescent state disappears via subcritical Andronov-Hopf bifurcation, and the periodic spiking disappears via saddle homoclinic orbit bifurcation. Simulations of the Morris-Lecar model. Parameters $g_{\mathrm{Ca}}=0.9, V_{4}=0.04, V_{3}(u)=0.08-u, I(u)=0.08-0.03 u$, where $\dot{u}=\mu(0.22+V)$ and $\mu=0.003$, the rest as in Fig. 59 . 


\section{"SubHopf/Homoclinic" Bursting}

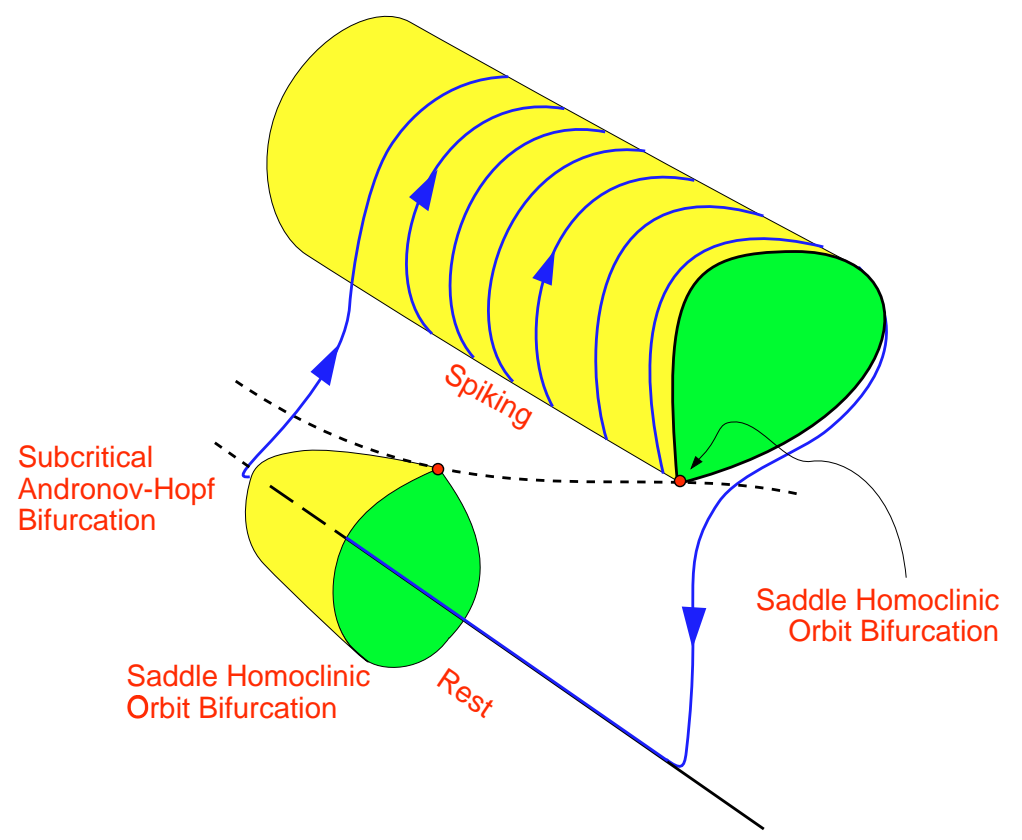

Fig. 70. "SubHopf/homoclinic" bursting: The quiescent state disappears via subcritical Andronov-Hopf bifurcation, and the periodic spiking disappears via saddle homoclinic orbit bifurcation. The same bifurcations form the "subHopf/homoclinic" hysteresis loop.

hysteresis loop, as shown in Fig. 71, or via "subHopf/homoclinic" hysteresis loop (not shown).

\subsubsection{4. "Hopf/circle" bursting}

The quiescent state disappears via supercritical Andronov-Hopf bifurcation, and the periodic limit cycle attractor corresponding to repetitive spiking disappears via saddle-node on invariant circle bifurcation. The bursting can occur via "fold/fold" hysteresis loop as shown in Fig. 72, or via any other point-point hysteresis loop from Fig. 55.

\subsubsection{5. "SubHopf/circle" bursting}

The quiescent state disappears via subcritical Andronov-Hopf bifurcation, and the periodic limit cycle attractor corresponding to repetitive spiking disappears via saddle-node on invariant circle bifurcation. The bursting can occur via "fold/fold" hysteresis loop as we illustrate in Fig. 73, or via "subHopf/fold" hysteresis loop; see Fig. 74.

\subsubsection{6. "Hopf/fold cycle" bursting}

The quiescent state disappears via supercritical Andronov-Hopf bifurcation, and the periodic limit cycle attractor corresponding to repetitive spiking disappears via fold limit cycle bifurcation; see Fig. 75. The bursting can also occur via "subHopf/fold cycle" hysteresis loop (not shown).

\subsubsection{7. "SubHopf/Hopf" bursting}

The quiescent state loses stability via subcritical Andronov-Hopf bifurcation, and the periodic limit cycle attractor corresponding to repetitive spiking shrinks to a point via supercritical Andronov-Hopf bifurcation. The bursting can occur via "fold/fold" hysteresis loop (Fig. 76) or "subHopf/fold" hysteresis loop (Fig. 77).

\subsubsection{8. "SubHopf/fold cycle" ("Elliptic") bursting}

The quiescent state loses stability via subcritical Andronov-Hopf bifurcation, and the periodic limit cycle attractor corresponding to repetitive spiking disappears via fold limit cycle bifurcation, as we illustrate in Fig. 78.

When the two bifurcations occur for nearby values of the slow variable, the fast subsystem is near Bautin bifurcation, and the burster has a local 


\section{"Hopf/Homoclinic" Bursting}
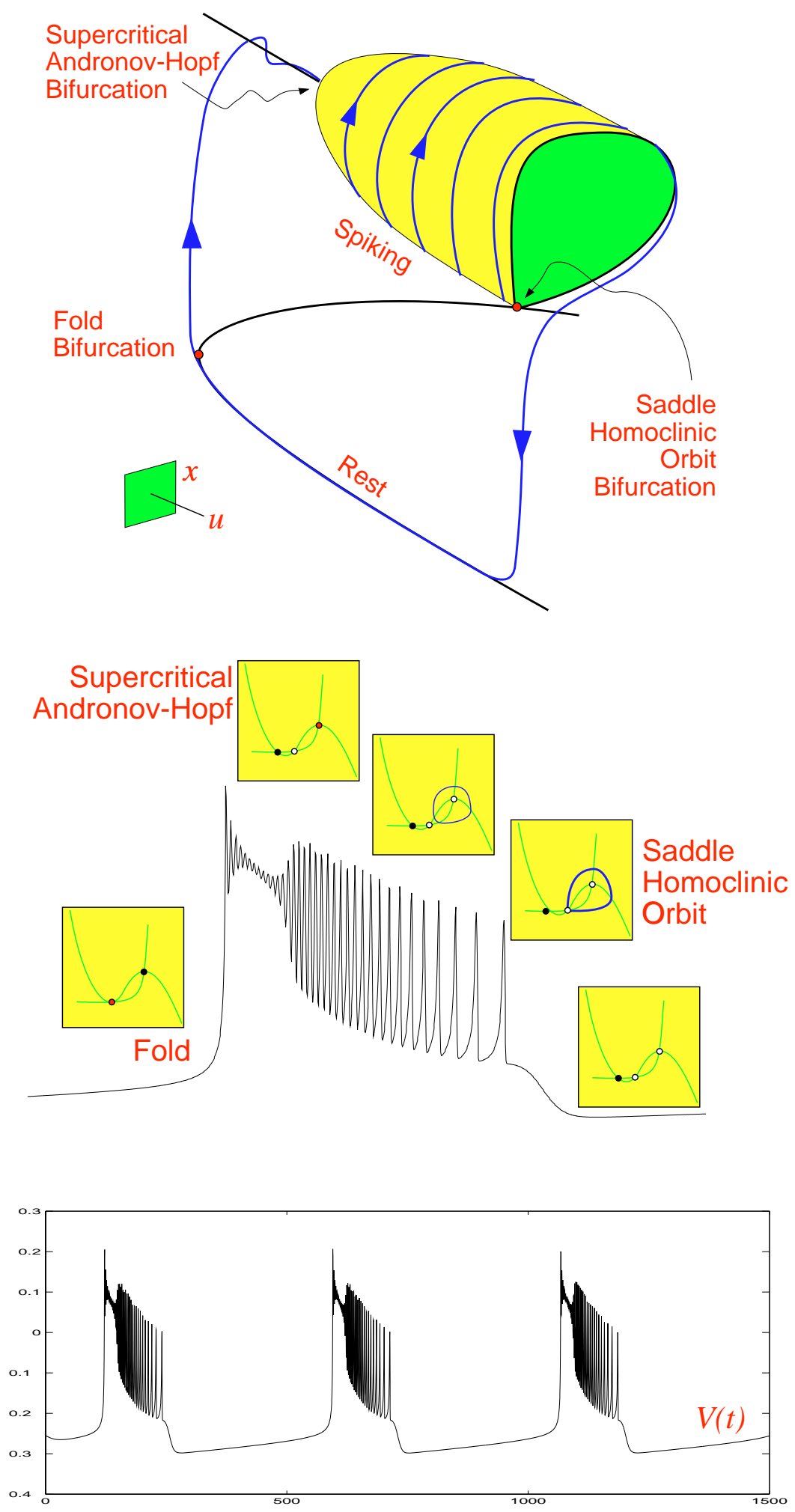

Fig. 71. "Hopf/homoclinic" bursting via "fold/homoclinic" hysteresis loop: The quiescent state disappears via supercritical Andronov-Hopf bifurcation, and the periodic spiking disappears via saddle homoclinic orbit bifurcation. Simulations of the Morris-Lecar model. Parameters as in Fig. 69, but $V_{4}=0.02$ and $\mu=0.01$. 


\section{"Hopf/Circle" Bursting}
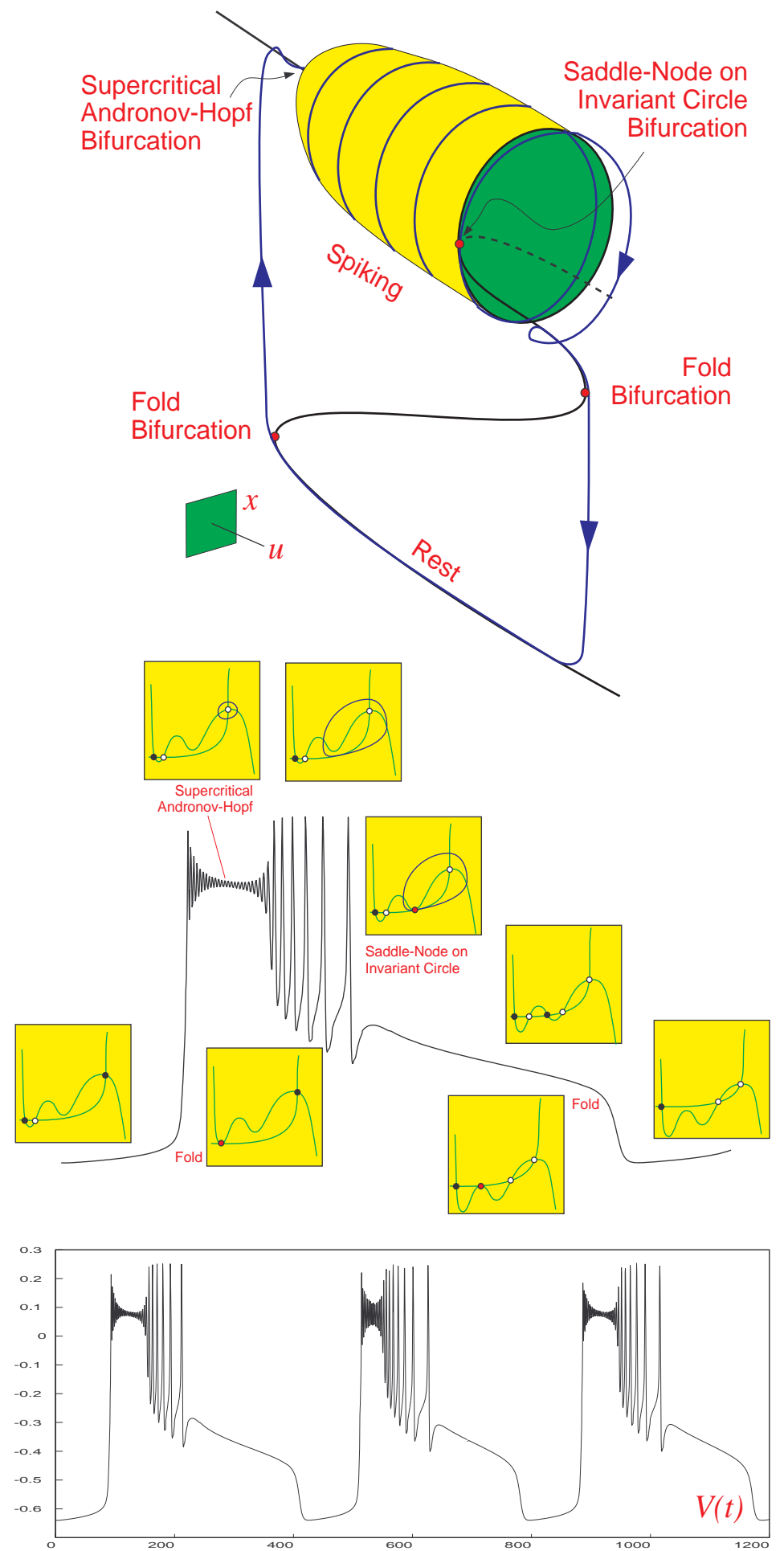

Fig. 72. "Hopf/circle" bursting via "fold/fold" hysteresis loop: The quiescent state disappears via supercritical AndronovHopf bifurcation, and the periodic spiking disappears via saddle-node on invariant circle bifurcation. 


\section{"SubHopf/Circle" Bursting}
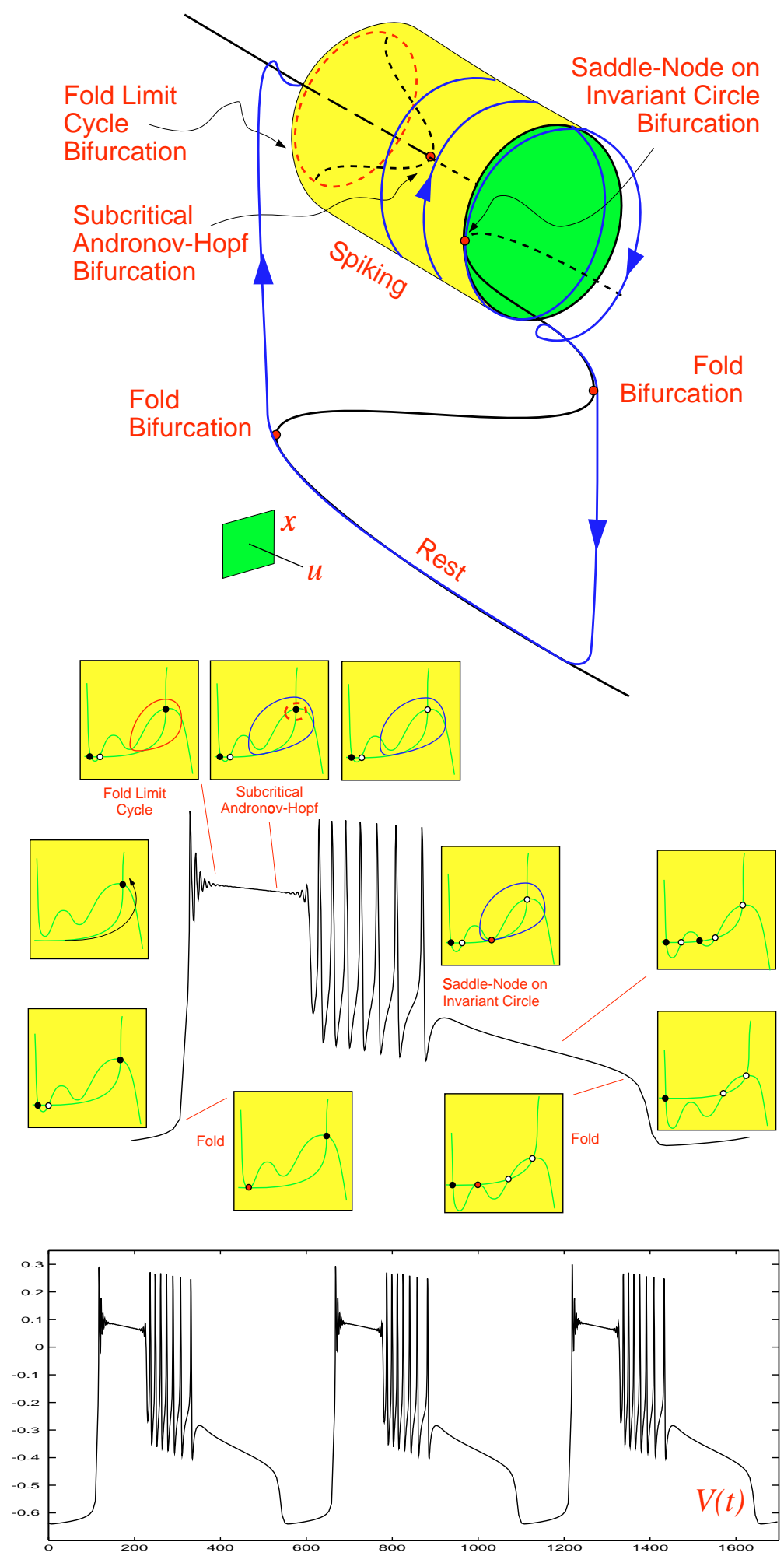

Fig. 73. "SubHopf/circle" bursting via "fold/fold" hysteresis loop: The quiescent state disappears via subcritical AndronovHopf bifurcation, and the periodic spiking disappears via saddle-node on invariant circle bifurcation. Simulations of the system from Fig. 61 with $V_{3}(u)=-0.135+0.3 u$ and $\mu=0.005$. 


\section{"SubHopf/Circle" Bursting}

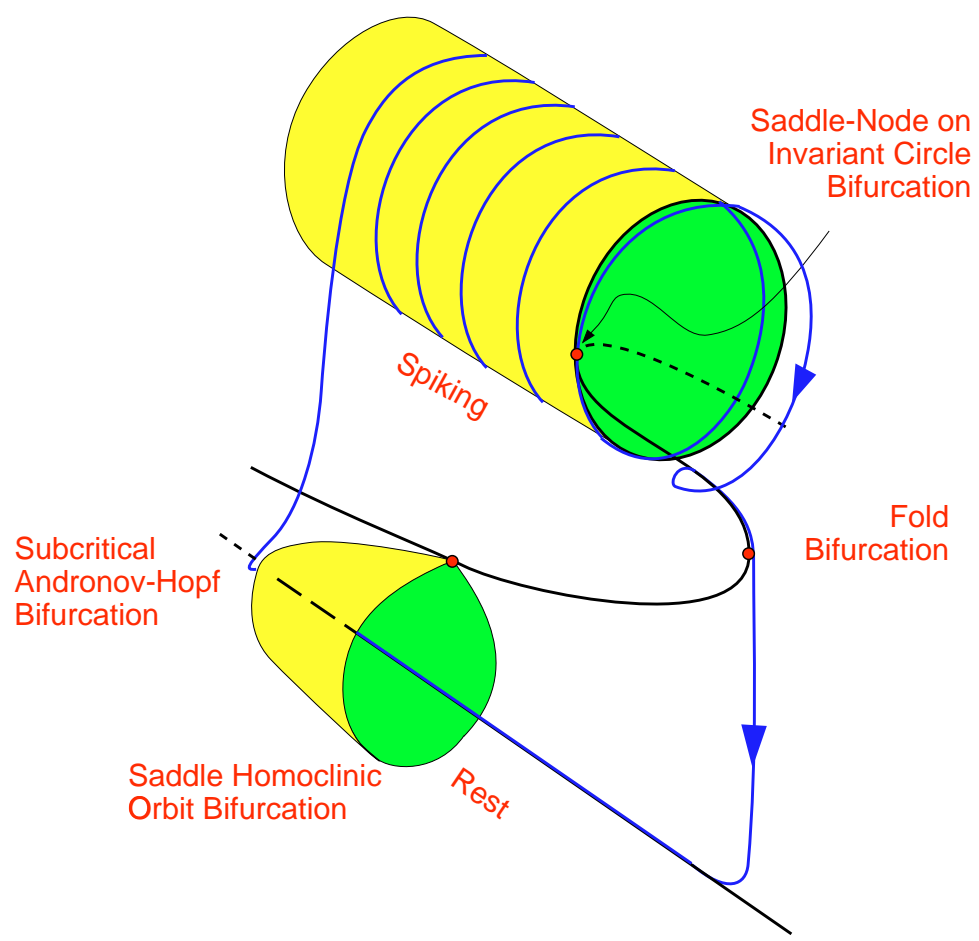

Fig. 74. "SubHopf/circle" bursting via "subHopf/fold" hysteresis loop: The quiescent state disappears via subcritical Andronov-Hopf bifurcation, and the periodic spiking disappears via saddle-node on invariant circle bifurcation.

canonical model [Izhikevich, 2000a]

$$
\begin{aligned}
& \dot{z}=(u+\mathrm{i} \omega) z+2 z|z|^{2}-z|z|^{4} \\
& \dot{u}=\mu\left(a-|z|^{2}\right)
\end{aligned}
$$

where $z \in \mathbb{C}$ and $u \in \mathbb{R}$ are the canonical fast and slow variables, respectively, and $a, \omega$ and $\mu \ll 1$ are parameters. The canonical model exhibits hysteresis loop periodic point-cycle bursting behavior for $0<a<1$; see Fig. 79. The bursting is frequently called "elliptic" due to the profile of the spiking amplitude.

Such a bursting occurs in rodent trigeminal interneurons [Del Negro et al. 1998], and it is exhibited by the FitzHugh-Rinzel model [Rinzel, 1987, see Figs. 80 and 81), Rush-Rinzel model [1994], Chay-Cook model [Bertram et al., 1995], Wu-Baer [1998] model, and Pernarowski [1994] polynomial model [de Vries, 1998]. Synchronization behavior of weakly connected "subHopf/fold cycle" bursters is scrutinized by Izhikevich [2000a]. We discuss it in Sec. 4.6.

Finally, the spiking limit cycle may not surround the rest state, as we illustrate in Fig. 82.

\subsubsection{9. "Hopf/Hopf" Bursting}

The rest state loses stability via supercritical Andronov-Hopf bifurcation, and the limit cycle attractor corresponding to repetitive spiking shrinks to a point also via supercritical Andronov-Hopf bifurcation; see Figs. 83. The bursting can occur via "fold/fold" hysteresis loop as we illustrate in Fig. 84, or via any other point-point hysteresis loop from Fig. 55.

When the quiescent state is the only attractor of a neural system, then one might assume that "Hopf/Hopf" bursting can only be of slow wave type; that is, the slow subsystem should be at least two-dimensional having a slow wave limit cycle (" $2+2$ " bursting). However, there is an example of " $2+1$ " hysteresis loop periodic "Hopf/Hopf" bursting having unique attractor for any value of the slow variable [Hoppensteadt \& Izhikevich, 1997, Sec. 2.9.4]; see Fig. 85 and detailed discussion by Izhikevich [1998].

Let us elaborate. Even though there is no coexistence of attractors at the supercritical AndronovHopf bifurcation, there may be a hysteresis loop due 


\section{"Hopf/Fold Cycle" Bursting}
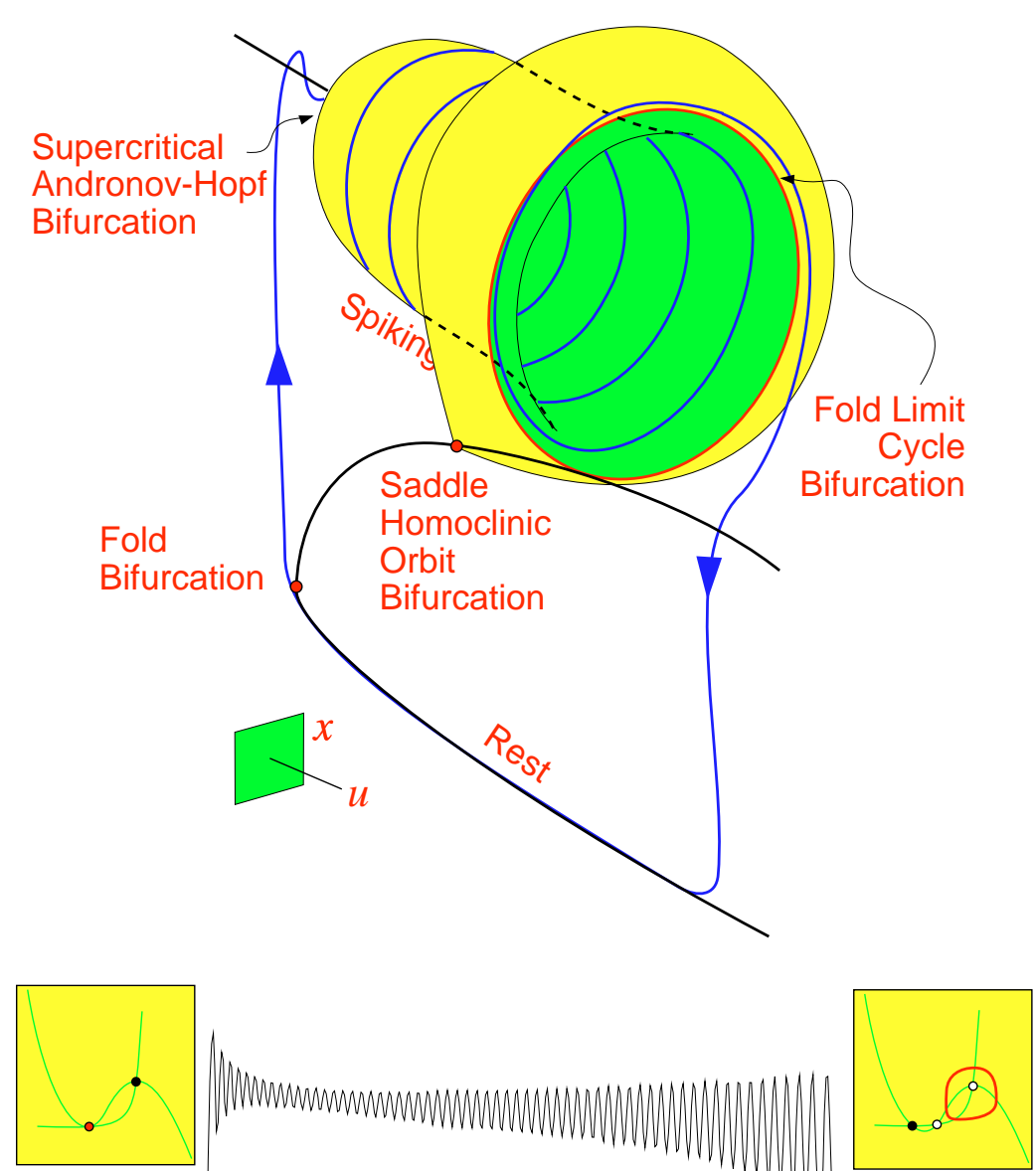

Fold

Supercritical Andronov-Hopf
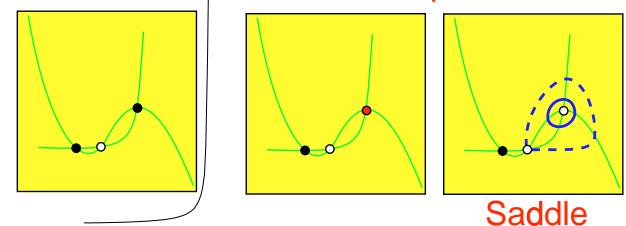

Saddle

Homoclinic

Orbit

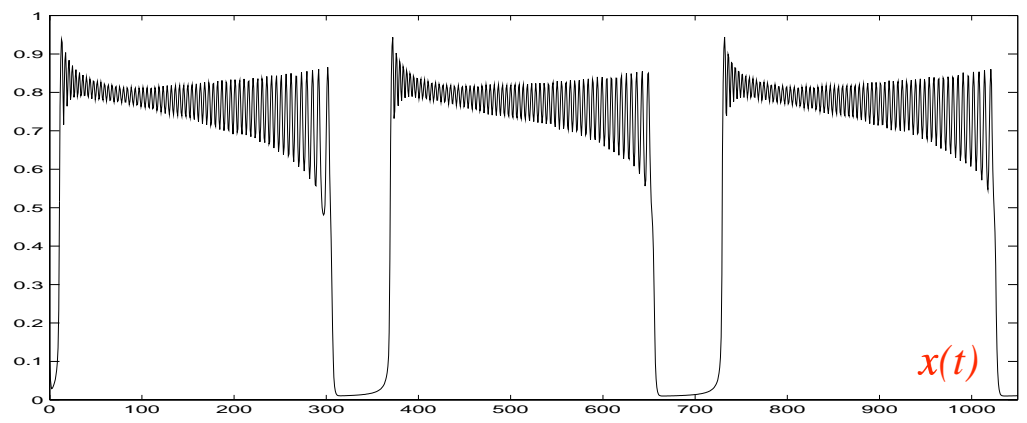

Fig. 75. "Hopf/fold cycle" bursting via "fold/fold cycle" hysteresis loop: The quiescent state disappears via supercritical Andronov-Hopf bifurcation, and the periodic spiking disappears via fold limit cycle bifurcation. Simulations of the WilsonCowan [1972] model with parameters as in Fig. 68 except $r_{\mathrm{y}}=-9.7, \mu=0.5$, and $\dot{u}=\mu(0.745-x) \sqrt{x}(u+0.05)$. 


\section{"SubHopf/Hopf" Bursting}
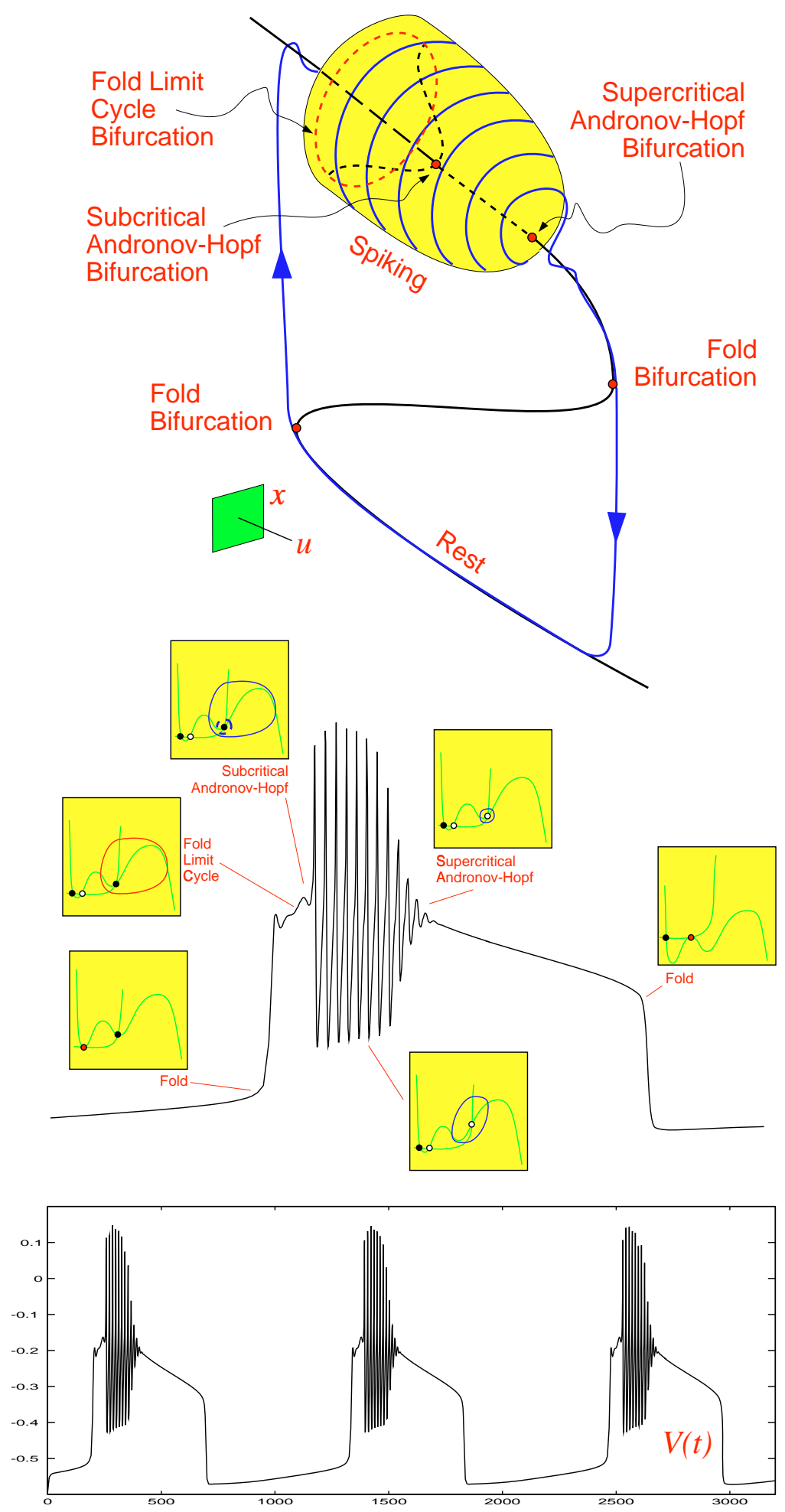

Fig. 76. "SubHopf/Hopf" bursting via "fold/fold" hysteresis loop: The quiescent state loses stability via subcritical AndronovHopf bifurcation, and the periodic spiking disappears via supercritical Andronov-Hopf bifurcation. 


\section{"SubHopf/Hopf" Bursting}

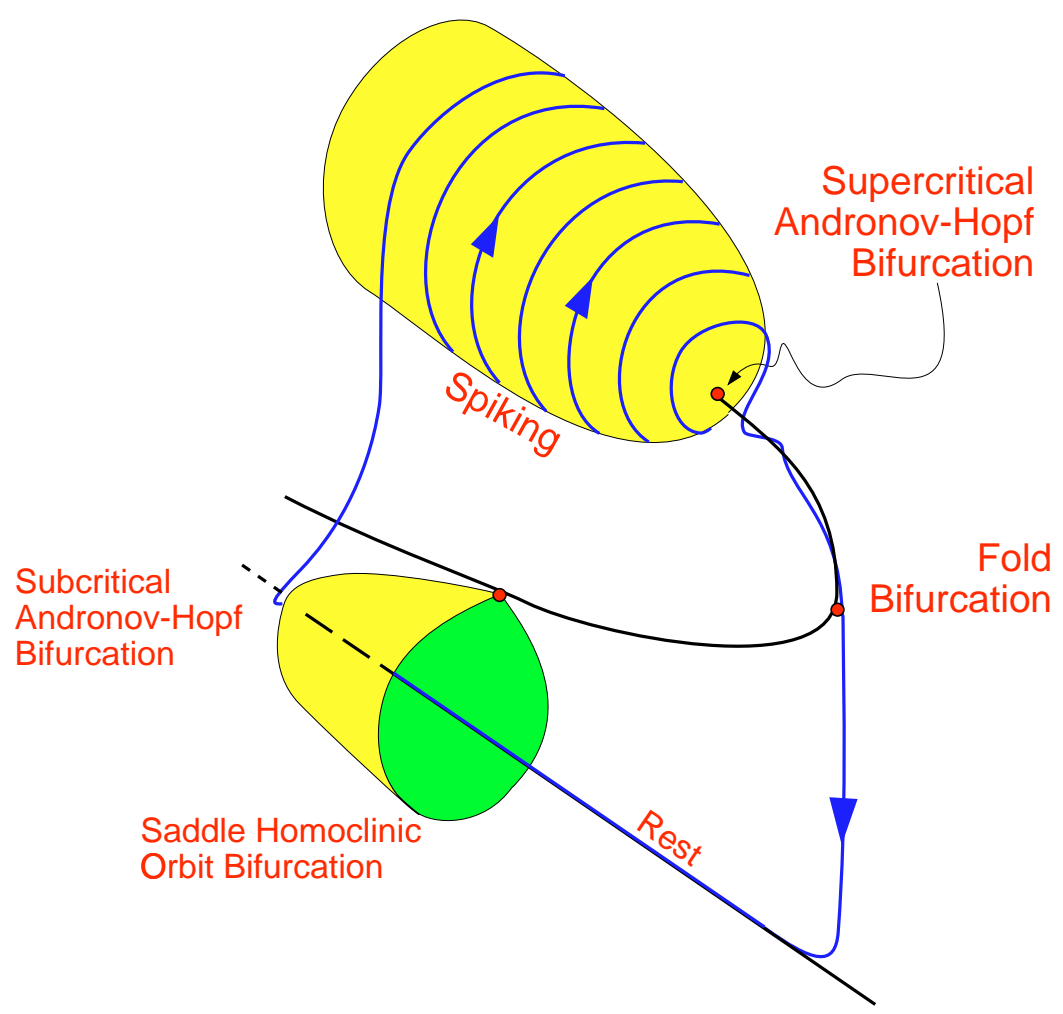

Fig. 77. "SubHopf/Hopf" bursting via "subHopf/fold" hysteresis loop: The quiescent state loses stability via subcritical Andronov-Hopf bifurcation, and the periodic spiking disappears via supercritical Andronov-Hopf bifurcation.

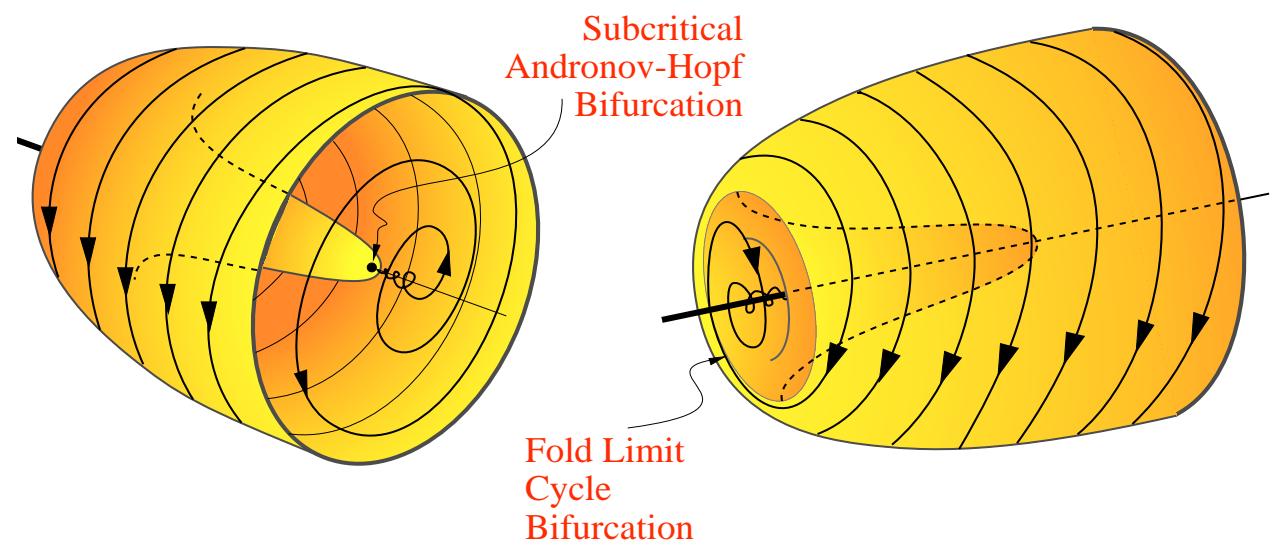

Fig. 78. "SubHopf/fold cycle" ("elliptic") bursting occurs when the rest state loses stability via subcritical AndronovHopf bifurcation, and the periodic attractor corresponding to spiking disappears via fold limit cycle bifurcation. The same bifurcations form the "subHopf/fold cycle" hysteresis loop. (Modified from [Hoppensteadt \& Izhikevich, 1997].) 

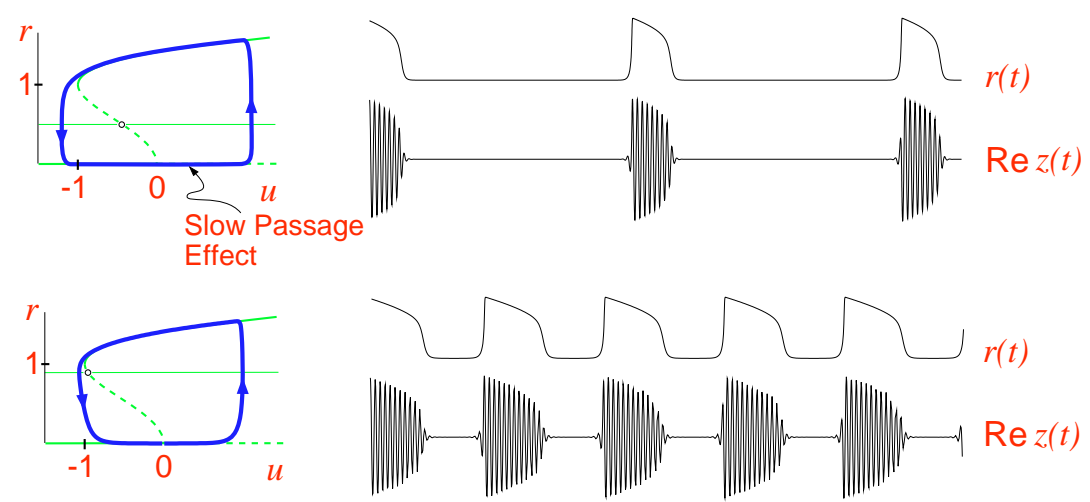

Fig. 79. Phase portrait and solution of the canonical model (28) for $\mu=0.1, \omega=3$, and $a=0.25$ (top) and $a=0.8$ (bottom). Modified from [Izhikevich, 2000a].
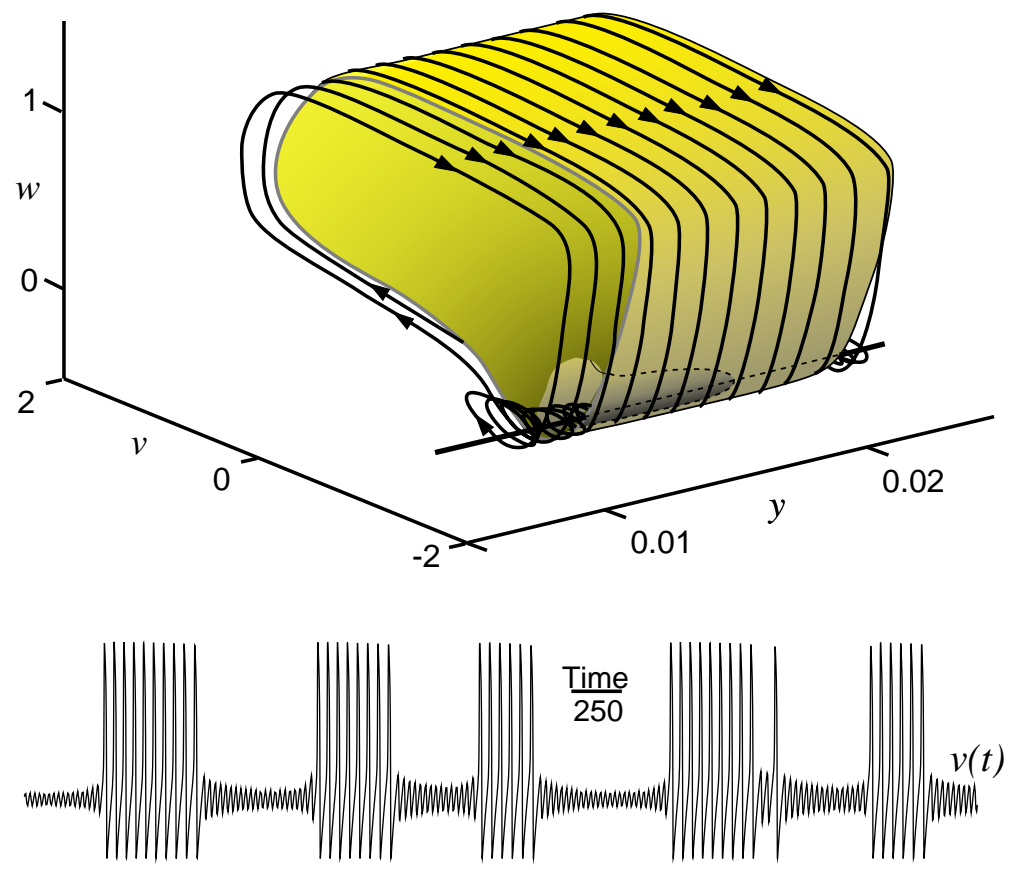

Fig. 80. FitzHugh-Rinzel model of "subHopf/fold cycle" bursting (from [Izhikevich, 2000a]).

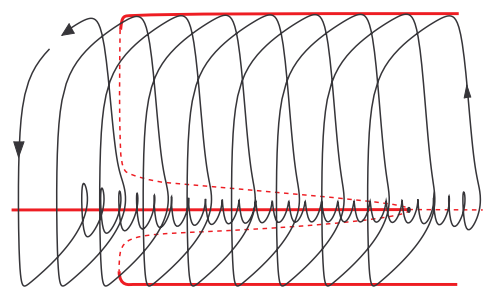

Slow Passage Effect

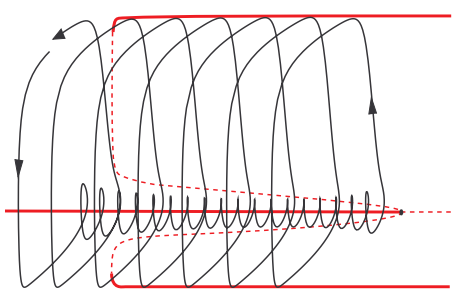

Premature Reentry

Fig. 81. Slow passage effect and premature reentry in the FitzHugh-Rinzel model of "subHopf/fold cycle" bursting. The latter likely contributes to the apparent irregularity of bursting (from [Izhikevich, 2000a]). 


\section{"SubHopf/Fold Cycle" Bursting}

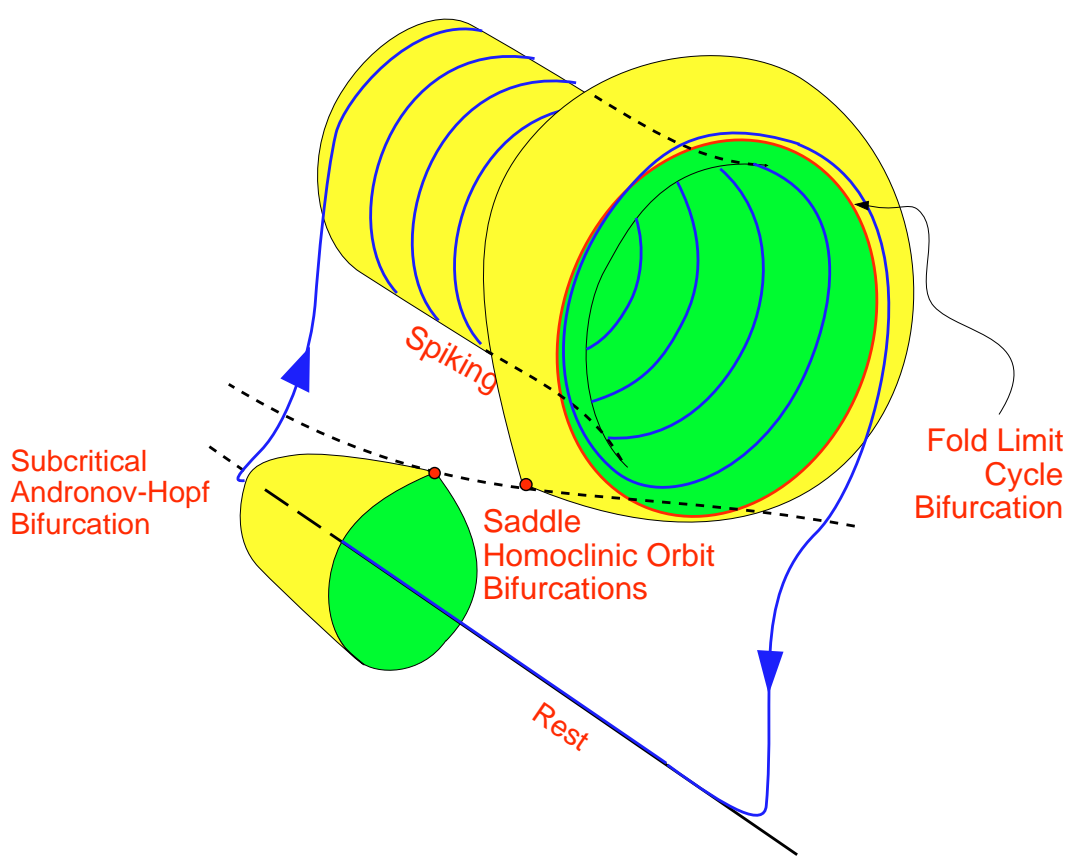

Fig. 82. "SubHopf/fold cycle" bursting occurs when the rest state loses stability via subcritical Andronov-Hopf bifurcation, and the periodic attractor corresponding to spiking disappears via fold limit cycle bifurcation. The rest state may not be inside the spiking limit cycle attractor.

Supercritical

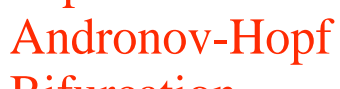

Bifurcation

\section{Supercritical Andronov-Hopf Bifurcation}

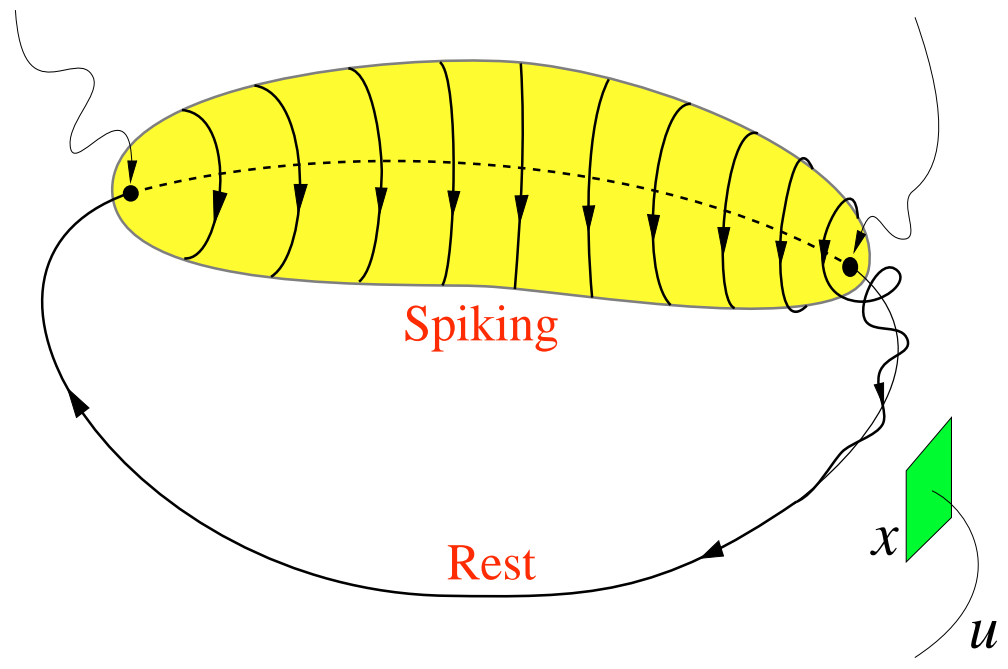

Fig. 83. "Hopf/Hopf" slow wave periodic burster: Rest state loses stability and the repetitive spiking disappears via supercritical Andronov-Hopf bifurcations. Slow variable $u \in \mathbb{S}^{1}$. 
"Hopf/Hopf" Bursting
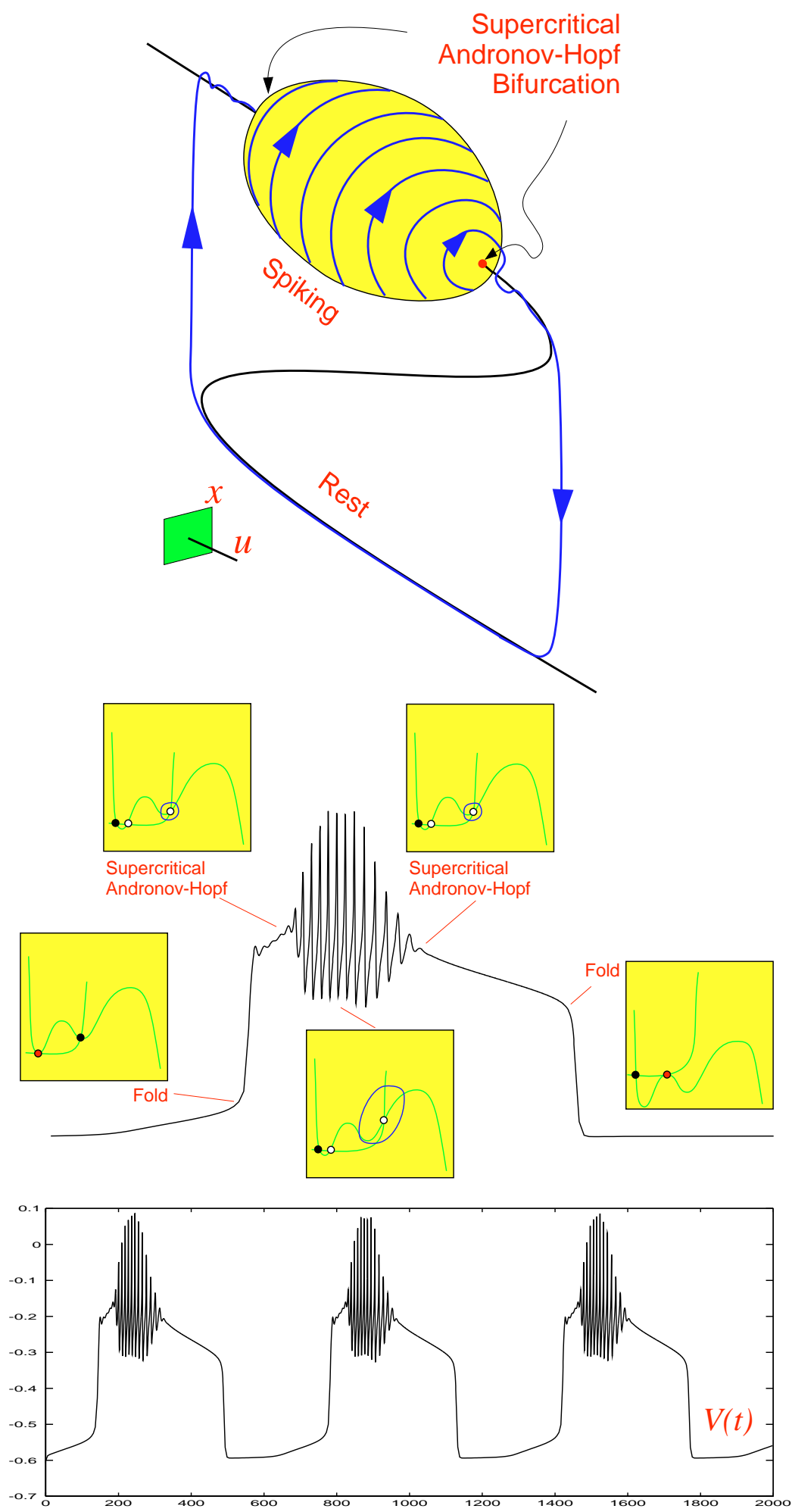

Fig. 84. "Hopf/Hopf" bursting via "fold/fold" hysteresis loop. Transitions between periodic spiking and quiescence occur via supercritical Andronov-Hopf bifurcations. 


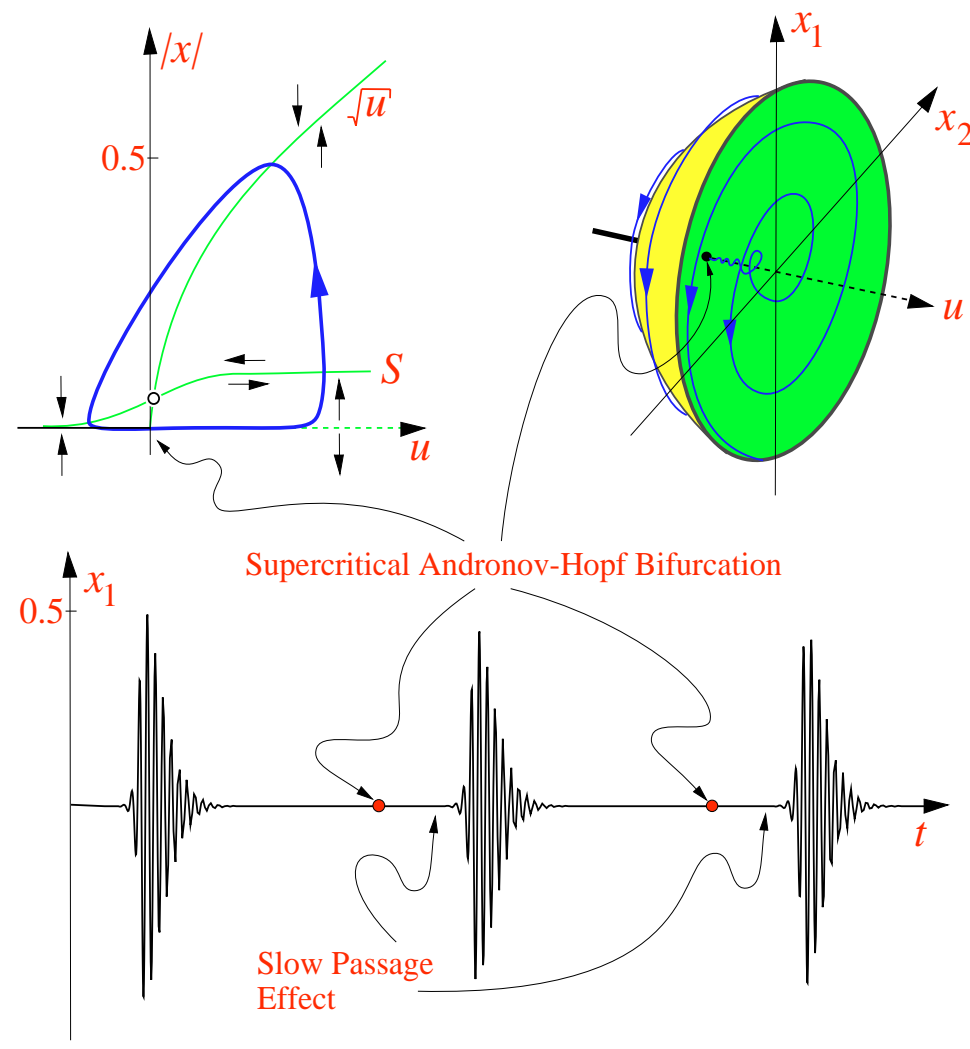

Fig. 85. "Hopf/Hopf" hysteresis loop periodic bursting in $\dot{x}=(u+\mathrm{i}) x-x|x|^{2}, \dot{u}=\mu(2 \lambda S(u / \lambda-\lambda)-|x|)$, where $x=$ $x_{1}+\mathrm{i} x_{2} \in \mathbb{C}, u \in \mathbb{R}, \mu=0.05, \lambda=\sqrt{\mu / 20}$ and $S(\rho)=1 /\left(1+e^{-\rho}\right)$. The hysteresis loop is due to the slow passage effect, not due to the coexistence of attractors (from [Hoppensteadt \& Izhikevich, 1997]). This burster is " $2+1$ ".

to the phenomenon knows as ramp effect, memory effect, or effect of slow passage through the bifurcation [Nejshtadt, 1985; Baer et al., 1989; Holden \& Erneux, 1993a, 1993b; Arnold et al., 1994]. The mechanism of supercritical elliptic bursting having one-dimensional slow subsystem can be explained as follows: When $x$ is close to a rest state, the variable $u$ increases and passes slowly through the bifurcation value. Due to the slow passage, the fast variable $x$ remains to be close to the rest equilibrium even after $u$ passed the bifurcation value. After a while $x$ diverges from the rest potential and starts to oscillate, in which case $u$ decreases (relatively quickly) and passes through the bifurcation in the opposite direction thereby completing the hysteresis loop, as in the upper-left corner of Fig. 85.

One should be warned though that the slow passage effect is very sensitive to nonsmoothness of the system and to noise. Thus, however ubiquitous this type of bursting can be in computer simulations, it may never be encountered experimentally.

The example of hysteresis loop "Hopf/Hopf" bursting depicted in Fig. 85 shows how cautious one should be when averaging bursting systems.
Indeed, if the fast subsystem has a unique attractor, and the slow subsystem is one-dimensional, then the averaged system (22) is one-dimensional, and hence cannot oscillate. Therefore hysteresis loop periodic bursting in (21) is impossible. This argument fails because (22) describes accurately (21) on the time scale of order $1 / \mu$ or less, unless other restrictions are imposed. But the time required for convergence of $x(t)$ to the unique attractor might be larger than $1 / \mu$ due to the slow passage effect. Moreover, the averaged system $(22)$ is not Lipchitz since $x(t, u)$ behaves like $\sqrt{u}$ near the Andronov-Hopf bifurcation point $u=0$. Thus, not only (22) is a bad approximation to (21), but also it has nonunique solutions. Therefore, averaging fails to provide good intuition for such bursting.

\subsubsection{Subtypes}

Let us use the "fold/homoclinic" bursting to illustrate the issue raised by Bertram et al. [1995]: The large amplitude limit cycle attractor corresponding to periodic spiking may encompass the quiescent state, which happens, e.g. during the big 


\section{"Fold/BIG Homoclinic" Bursting}
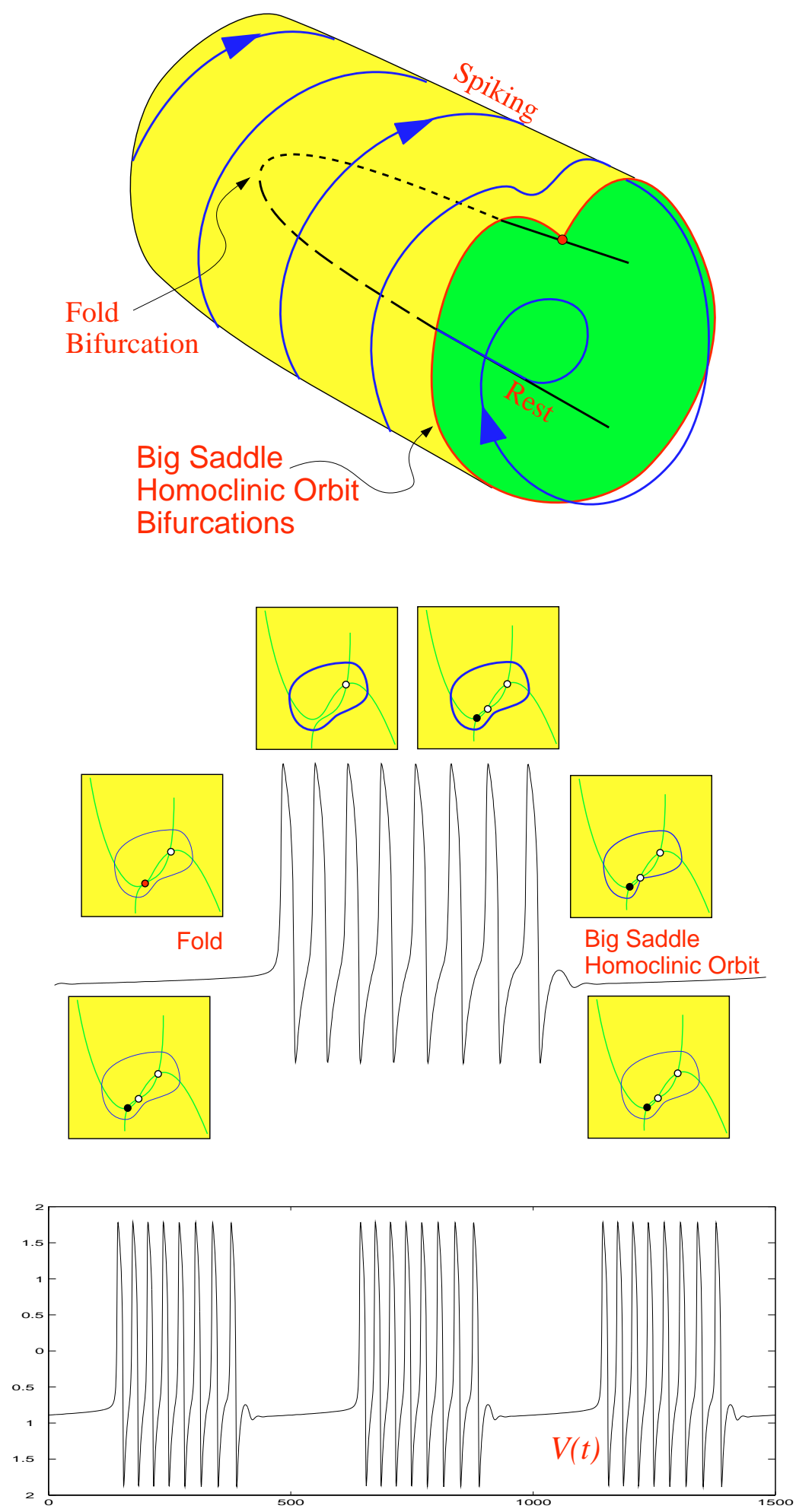

Fig. 86. "Fold/big homoclinic" ("square-wave") bursting: The rest state disappears via fold bifurcation and the limit cycle disappears via big saddle homoclinic orbit bifurcation. Simulations of (15) with $a(u)=-u, b=1.75, c=-0.5, d=0.1$, $\varepsilon=0.15$, and the slow subsystem $\dot{u}=\mu(0.5+v)$ with $\mu=0.0005$. 


\section{"Circle/Circle" Bursting}

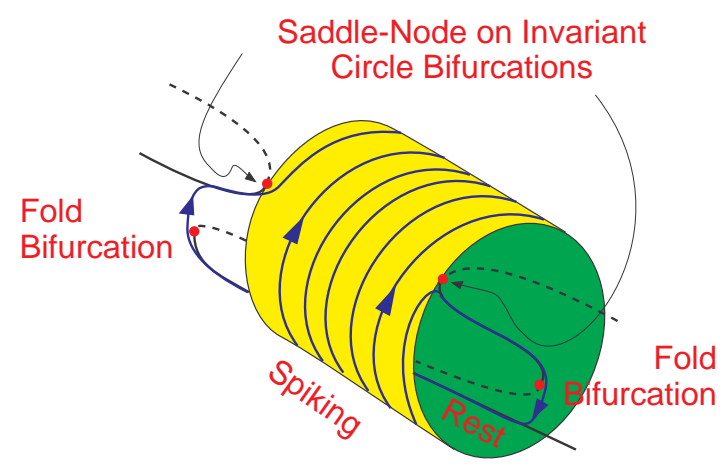

"Circle/BIG Homoclinic" Bursting

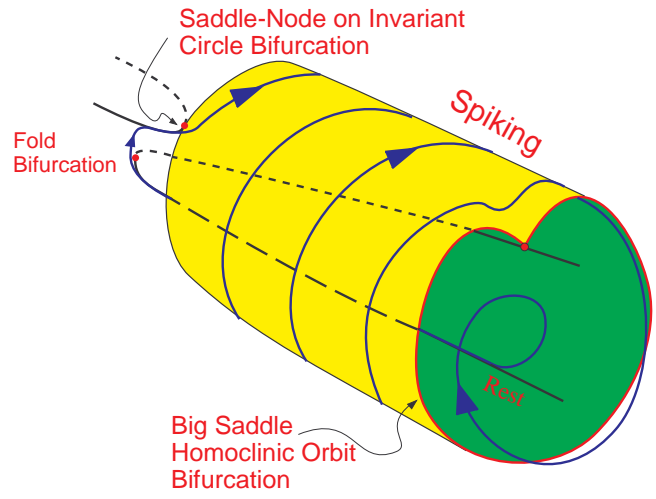

"SubHopf/BIG Homoclinic" Bursting

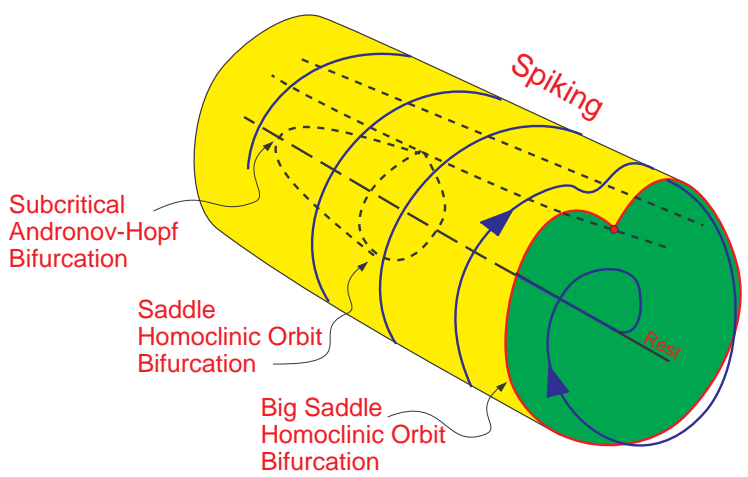

"SubHopf/Circle" Bursting

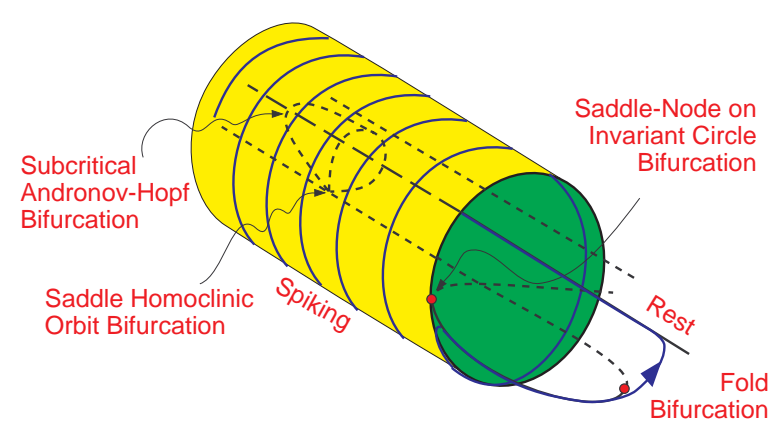

"Fold/Circle" Bursting

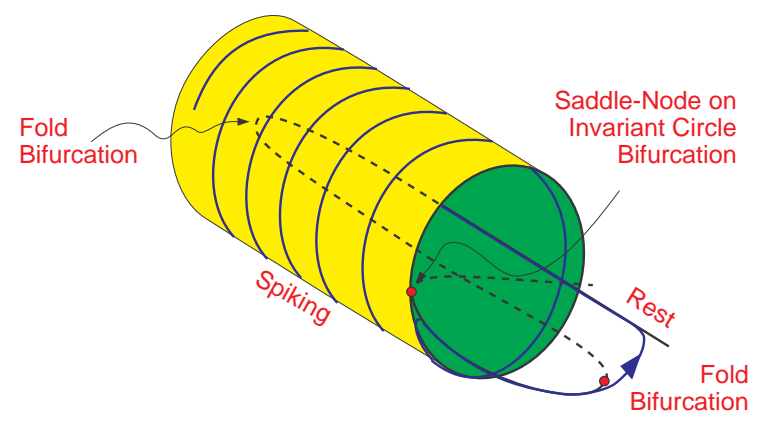

"Fold/Fold Cycle" Bursting

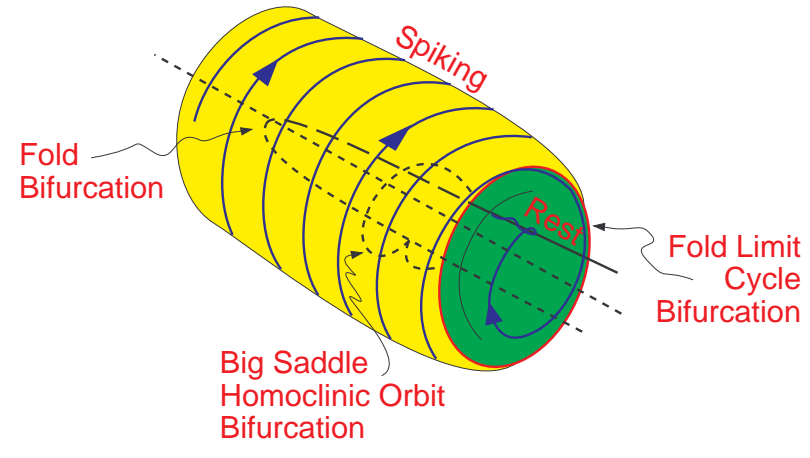

Fig. 87. The large amplitude limit cycle attractor corresponding to repetitive spiking can surround the rest state. 


\section{"Homoclinic/BIG Homoclinic" Bursting}
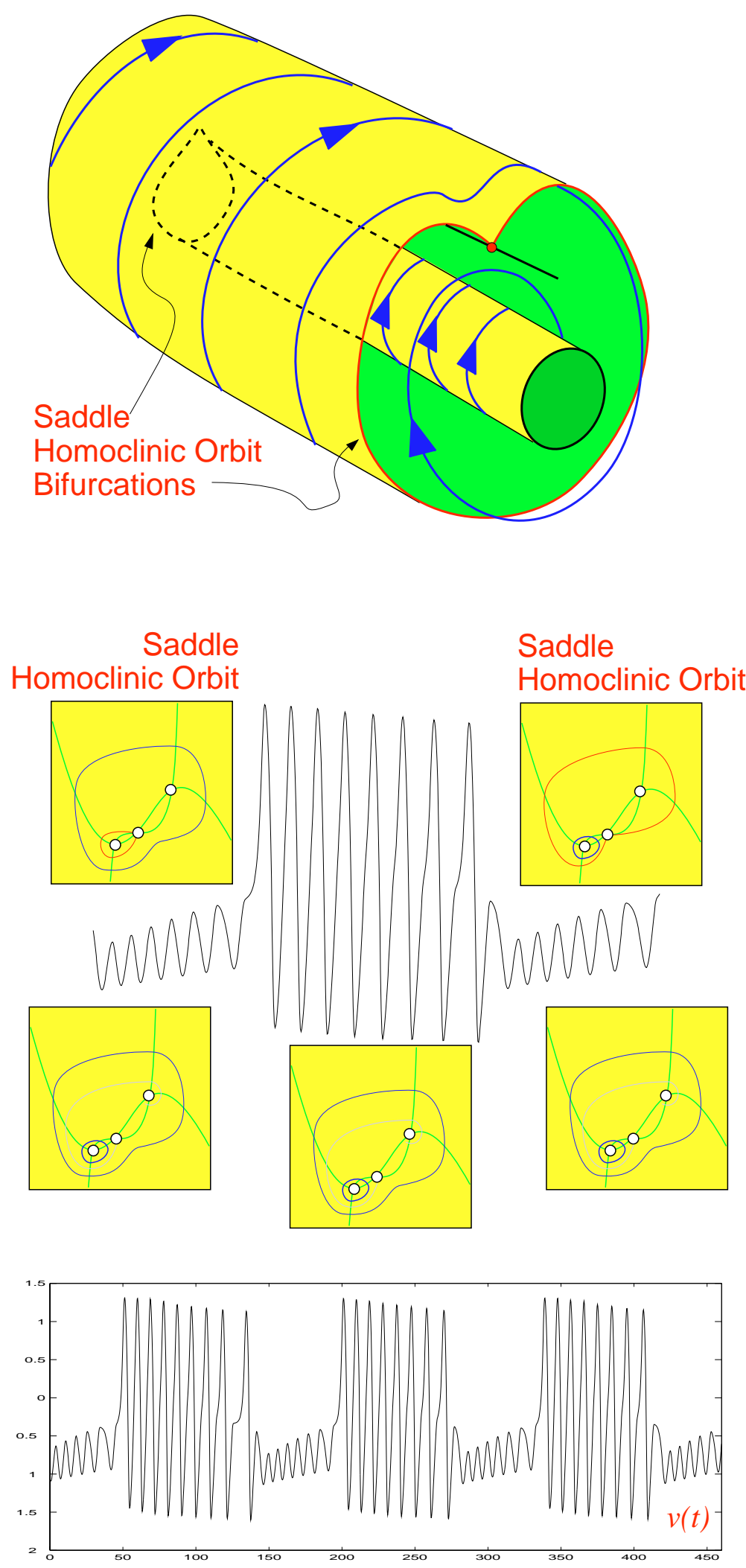

Fig. 88. "Homoclinic/homoclinic" cycle-cycle bursting: The transitions between quiescent and spiking limit cycles occur via saddle homoclinic orbit bifurcations. The same bifurcations form the hysteresis loop. Simulations of $(15)$ with $a(u)=-u$, $b=1.3, c=-0.32, d=0.05, \varepsilon=1$, slow subsystem $\dot{u}=\mu(0.4+v)$ and $\mu=0.01$. 


\section{"Homoclinic/Homoclinic" Bursting}

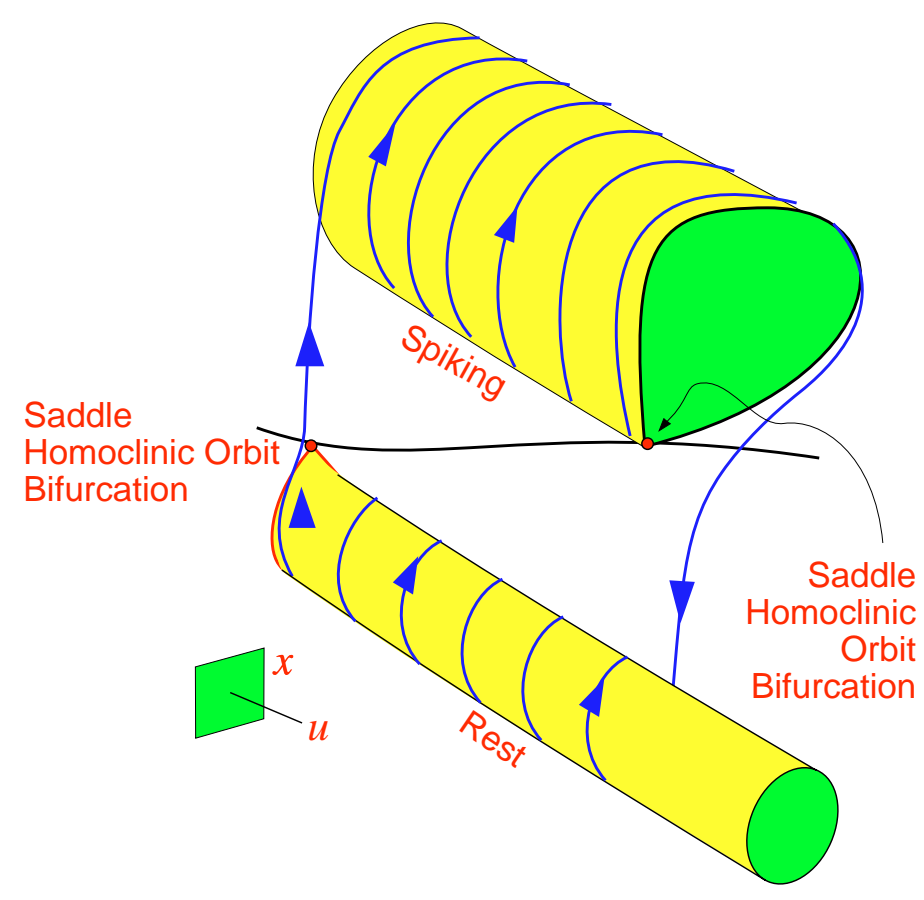

Fig. 89. "Homoclinic/homoclinic" cycle-cycle bursting: The transitions between quiescent and spiking limit cycles occur via saddle homoclinic orbit bifurcations, which form a hysteresis loop.

saddle homoclinic orbit bifurcation; see Figs. 32 and 86. Bertram et al. [1995] suggested to refer to such "fold/homoclinic" bursting as being Type Ib, as opposed to "regular" Type Ia. The "fold/big homoclinic" bursting is not an exception: Other bursters may have the same property, as we illustrate in Fig. 87. Even though the new types are equivalent to the old ones from the purely mathematical point of view, it may still be useful to distinguish between them, since they might have different neurocomputational properties.

Finally, the distinction may have limited value when the fast subsystem has dimension 3 and up, since the notions surround or in between may not be well defined there.

\subsection{Planar Cycle-cycle bursting}

Next, we give examples of some cycle-cycle planar bursters.

\subsection{1. "Homoclinic/homoclinic" bursting}

The transitions from quiescent to spiking limit cycles and back occur via saddle homoclinic orbit bifurcations; see Figs. 88 and 89.

\subsection{2. "Homoclinic/Hopf" bursting}

The quiescent limit cycle disappears via saddle homoclinic orbit bifurcation and the spiking limit cycle shrinks to a point via supercritical AndronovHopf bifurcation; see Fig. 90.

\subsection{3. "Homoclinic/fold cycle" bursting}

The quiescent limit cycle disappears via saddle homoclinic orbit bifurcation and the spiking limit cycle disappears via fold limit cycle bifurcation; see Fig. 91.

\subsection{4. "Homoclinic/circle" bursting}

The quiescent limit cycle disappears via saddle homoclinic orbit bifurcation and the spiking limit cycle disappears via saddle-node on invariant circle bifurcation; see Fig. 92.

\subsection{5. "Fold cycle/fold cycle" bursting}

The transitions between quiescent and spiking limit cycle attractors occur via fold limit cycle bifurcations; see Figs. 93 and 94. This is the only type of 


\section{"Homoclinic/Hopf" Bursting}
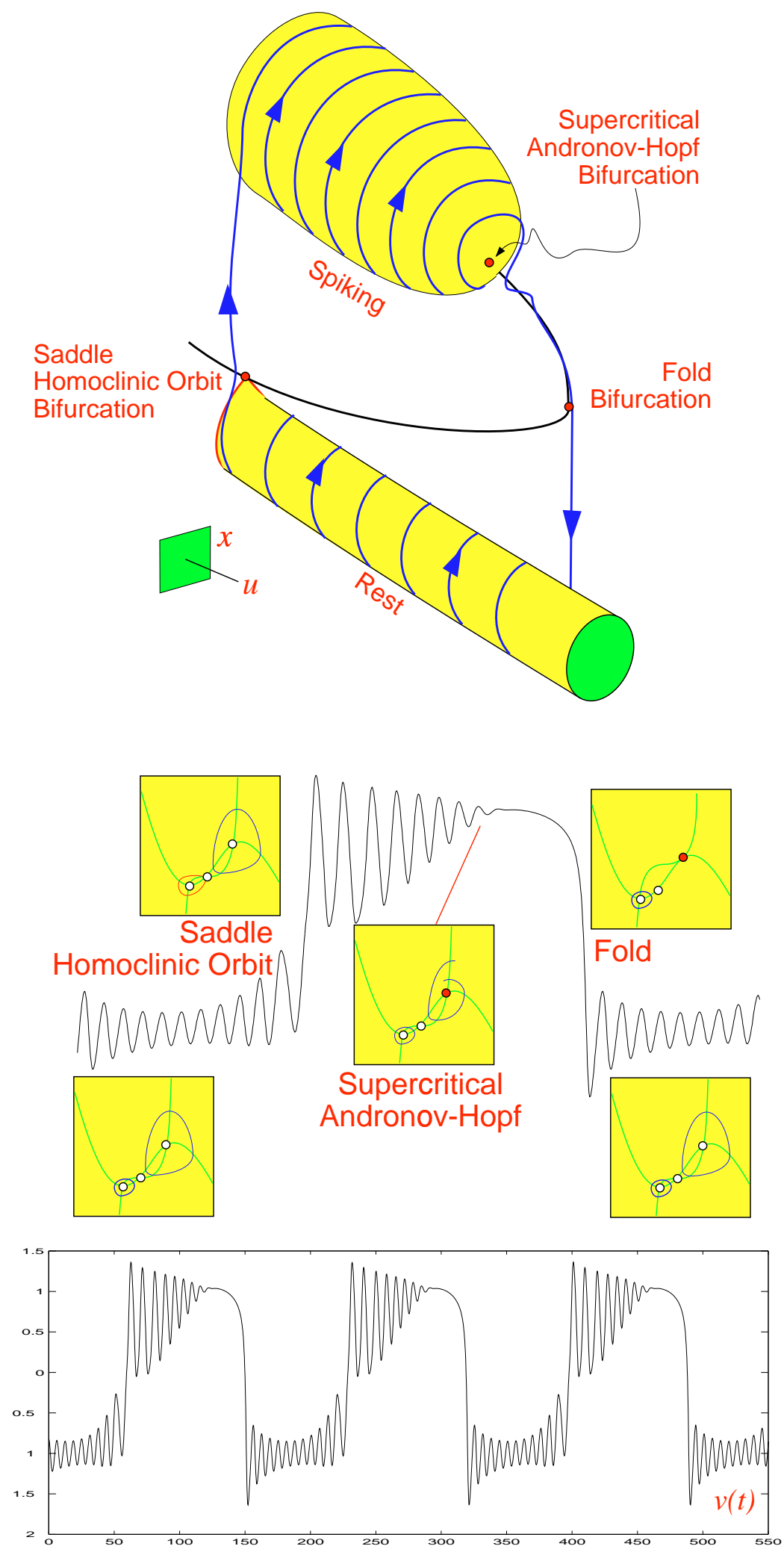

Fig. 90. "Homoclinic/Hopf" cycle-cycle bursting via "homoclinic/fold" hysteresis loop: The quiescent oscillation disappears via saddle homoclinic orbit bifurcation and the periodic spiking disappears via supercritical Andronov-Hopf bifurcation. Simulations of $(15)$ with $a(u)=-0.77-0.33 u /(u+0.15), b(u)=1.65+u, c=-0.15+u, d=0.1, \varepsilon=1$, slow subsystem $\dot{u}=\mu v$ and $\mu=0.01$. 


\section{"Homoclinic/Fold Cycle" Bursting}

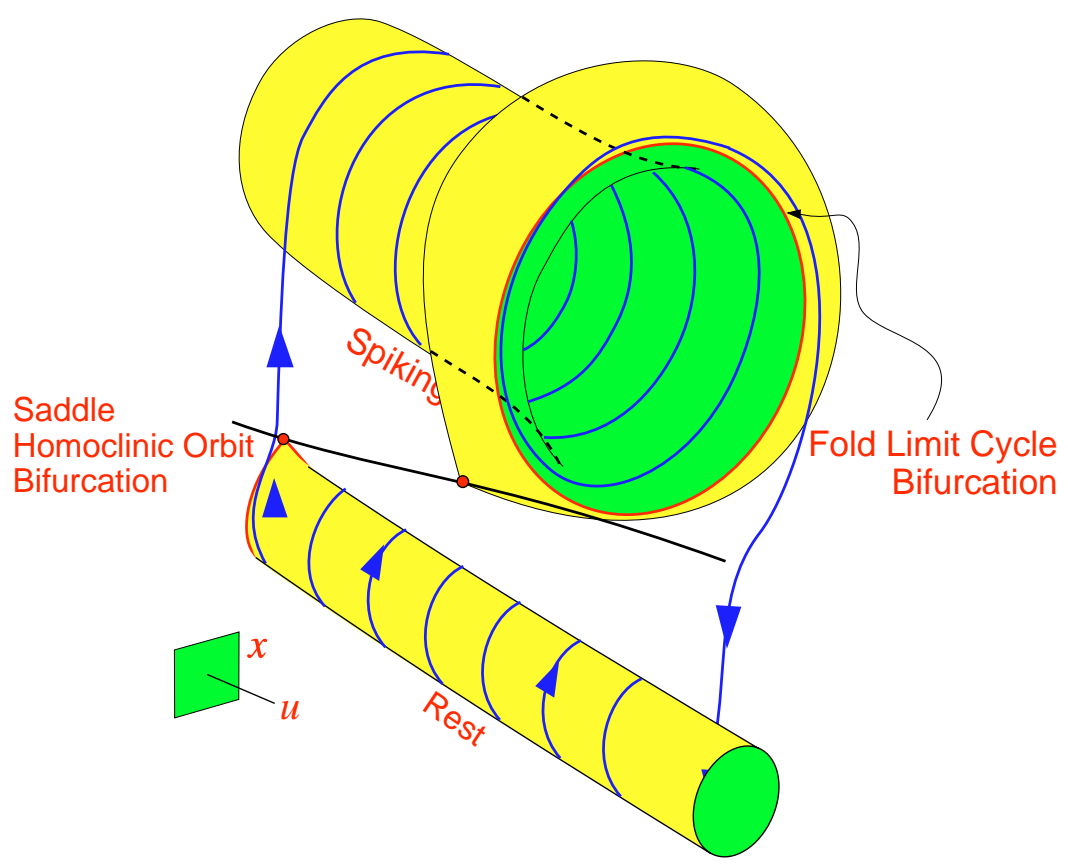

Fig. 91. "Homoclinic/fold cycle" bursting: The quiescent oscillation disappears via saddle homoclinic orbit bifurcation and the periodic spiking disappears via fold limit cycle bifurcation. The same bifurcations form the hysteresis loop.

\section{"Homoclinic/Circle" Bursting}

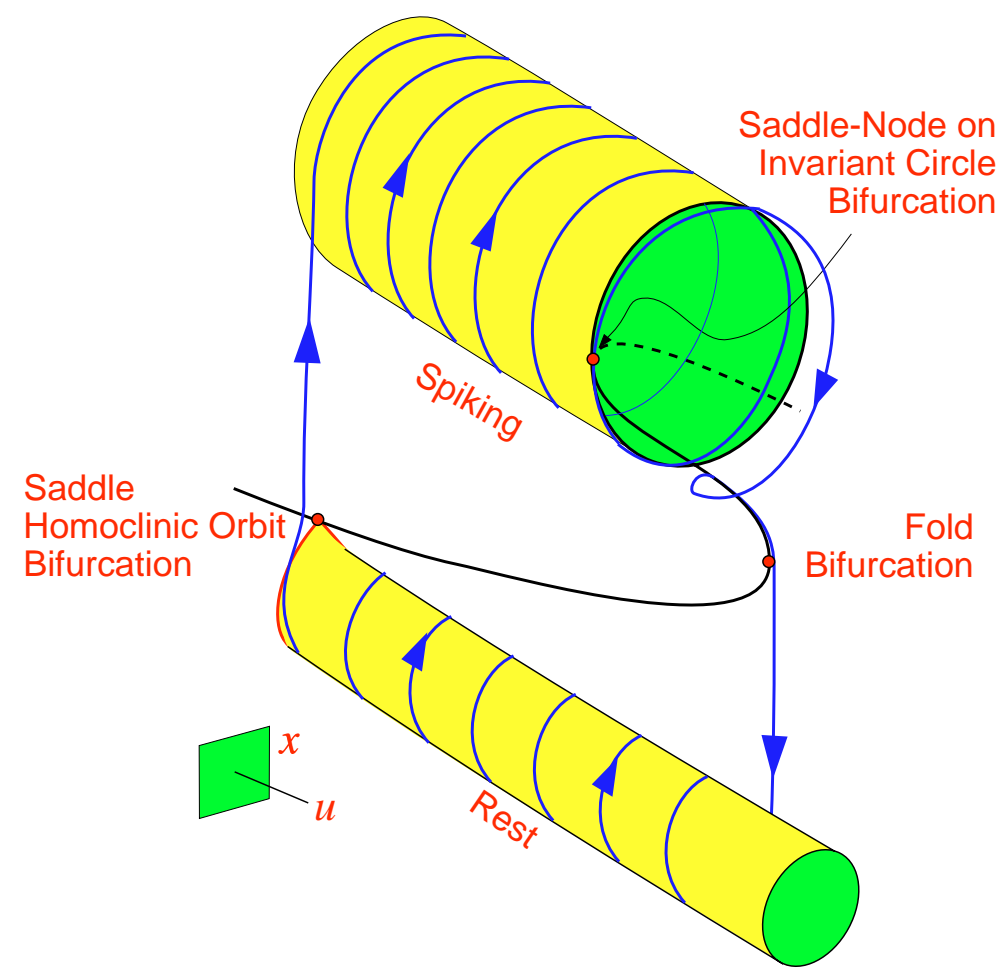

Fig. 92. "Homoclinic/circle" cycle-cycle bursting via "homoclinic/fold" hysteresis loop: The quiescent oscillation disappears via saddle homoclinic orbit bifurcation and the periodic spiking disappears via saddle-node on invariant circle bifurcation. 


\section{"Fold Cycle/Fold Cycle" Bursting}

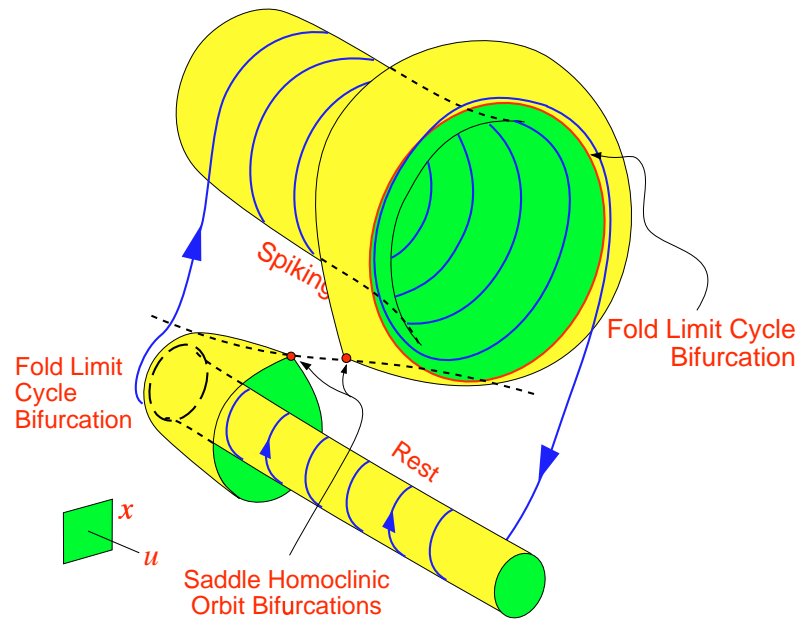

Fig. 93. "Fold cycle/fold cycle" bursting: The transitions between quiescent and spiking oscillations occur via fold limit cycle bifurcations, which form a hysteresis loop.

\section{"Fold Cycle/Fold Cycle" Bursting}

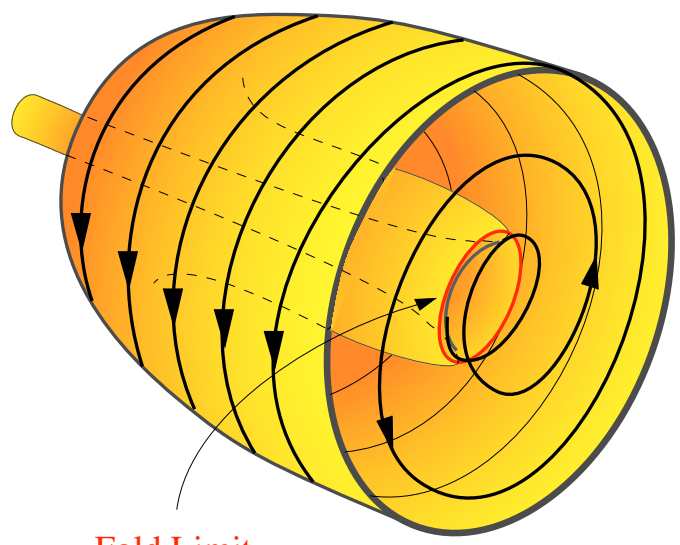

Fold Limit

Cycle

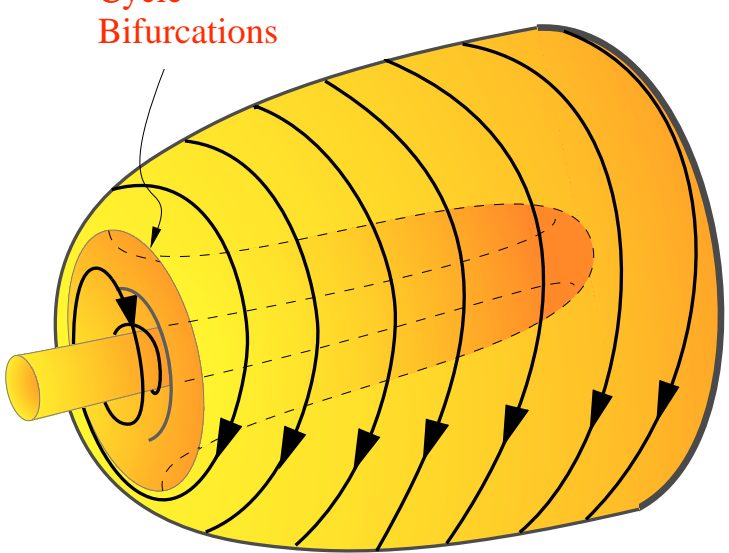

Fig. 94. "Fold cycle/fold cycle" bursting: The transitions between quiescent and spiking oscillations occur via fold limit cycle bifurcations, which form a hysteresis loop.

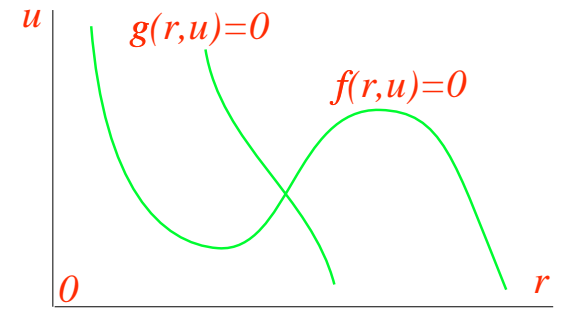

Fig. 95. Typical intersections of nullclines in "fold cycle/fold cycle" hysteresis loop bursting: $r>0$ is the amplitude of fast oscillation, $u$ is the slow variable.

cycle-cycle bursting for which rigorous asymptotic theory exists [Mishchenko et al., 1994]. "Fold cycle/fold cycle" bursting can be seen in the Hodgkin-Huxley-like models by Shorten and Wall [2000] and Wang [1993]. The latter also exhibits "fold cycle/flip" bursting.

An easy way to make a simple example of "fold cycle/fold cycle" burster is to take any standard relaxation system

$$
\begin{aligned}
& \dot{r}=f(r, u) \\
& \dot{u}=\mu g(r, u)
\end{aligned}
$$

having $N$-shaped fast nullcline that intersects the slow nullcline at one point (see Fig. 95) and assume that $r$ is the amplitude of fast oscillation. The variable $z(t)=r(t) e^{\mathrm{i} \omega t}$ would exhibit "fold cycle/fold cycle" hysteresis loop bursting. Thus, most of the results devoted to studying synchronization properties of relaxation oscillators (see e.g. [Belair \& Holmes, 1984; Storti \& Rand, 1986; Grasman, 1987; Somers \& Kopell, 1993, 1995; Kopell \& Somers, 1995; Terman \& Wang, 1995; Terman \& Lee, 1997; Izhikevich, 2000b]) can immediately be generalized to that of "fold cycle/fold cycle" bursters.

There is a relationship between the "fold cycle/fold cycle" and "subHopf/fold cycle" ("elliptic") bursting: When the latter has a fast subsystem near Bautin bifurcation and it receives weak time-dependent input, it is described by the canonical model [Izhikevich, 1999]

$$
\begin{aligned}
\dot{r} & =b+r+2 r^{3}+r^{5} \\
\dot{\varphi} & =\omega \\
\dot{u} & =\mu\left(a-r^{2}\right)
\end{aligned}
$$

where $z=r e^{\mathrm{i} \varphi} \in \mathbb{C}$, which coincides with (28) when $b=0$. Its typical "fold cycle/fold cycle" bursting solution is depicted in Fig. 96. 


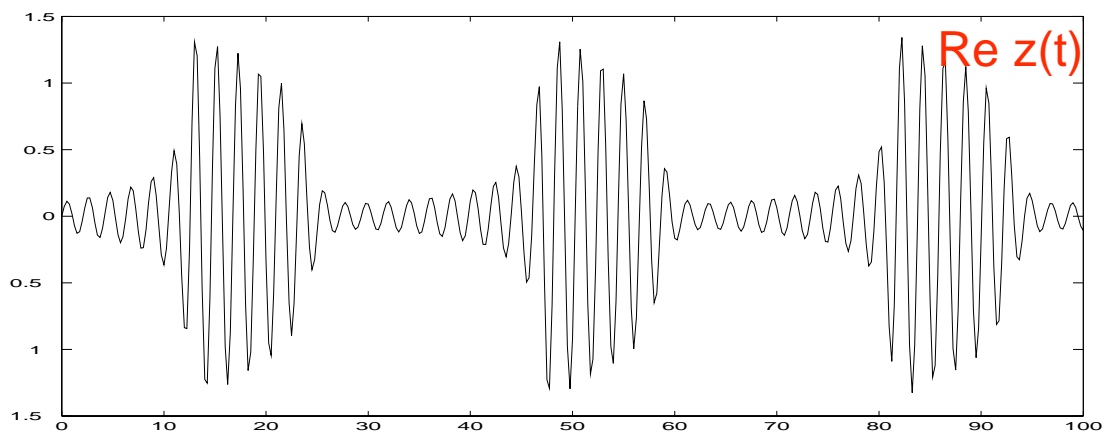

Fig. 96. A typical "fold cycle/fold cycle" bursting solution of (29). Parameters: $a=0.5, b=0.1, \omega=3, \mu=0.1$.

\section{"Fold Cycle/Homoclinic" Bursting}

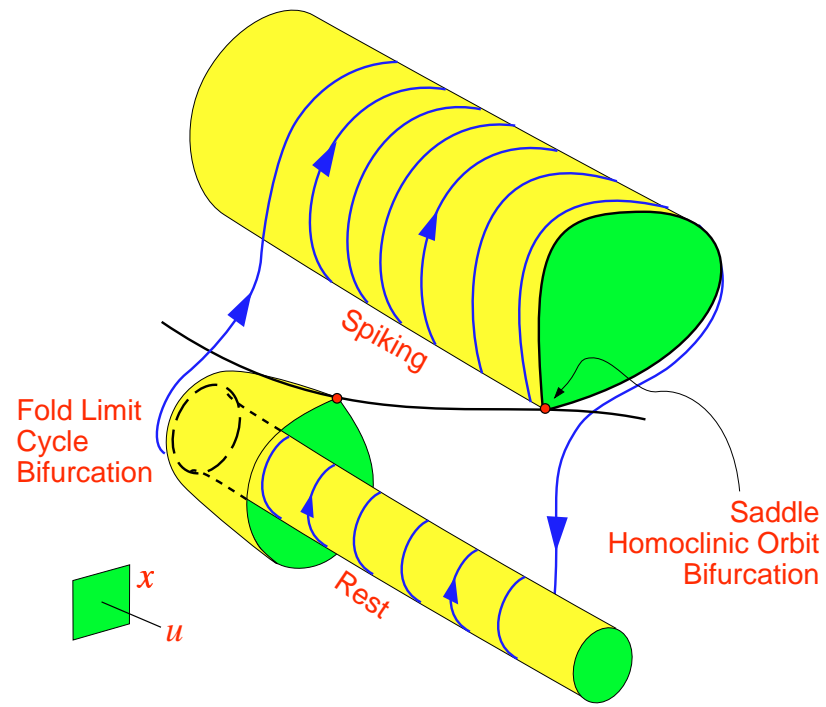

Fig. 97. "Fold cycle/homoclinic" cycle-cycle bursting: The quiescent state disappears via fold limit cycle bifurcation and the spiking state disappears via saddle homoclinic orbit bifurcation. The same bifurcations form the hysteresis loop.

\subsection{6. "Fold cycle/homoclinic" bursting}

The quiescent oscillation disappears via fold limit cycle bifurcation and the spiking oscillation disappears via saddle homoclinic orbit bifurcation; see Fig. 97.

\subsection{7. "Fold cycle/circle" bursting}

The quiescent oscillation disappears via fold limit cycle bifurcation and the spiking oscillation disappears via saddle-node on invariant circle bifurcation; see Fig. 98.

\subsection{8. "Fold cycle/Hopf" bursting}

The quiescent oscillation disappears via fold limit cycle bifurcation and the spiking oscillation shrinks

\section{"Fold Cycle/Circle" Bursting}

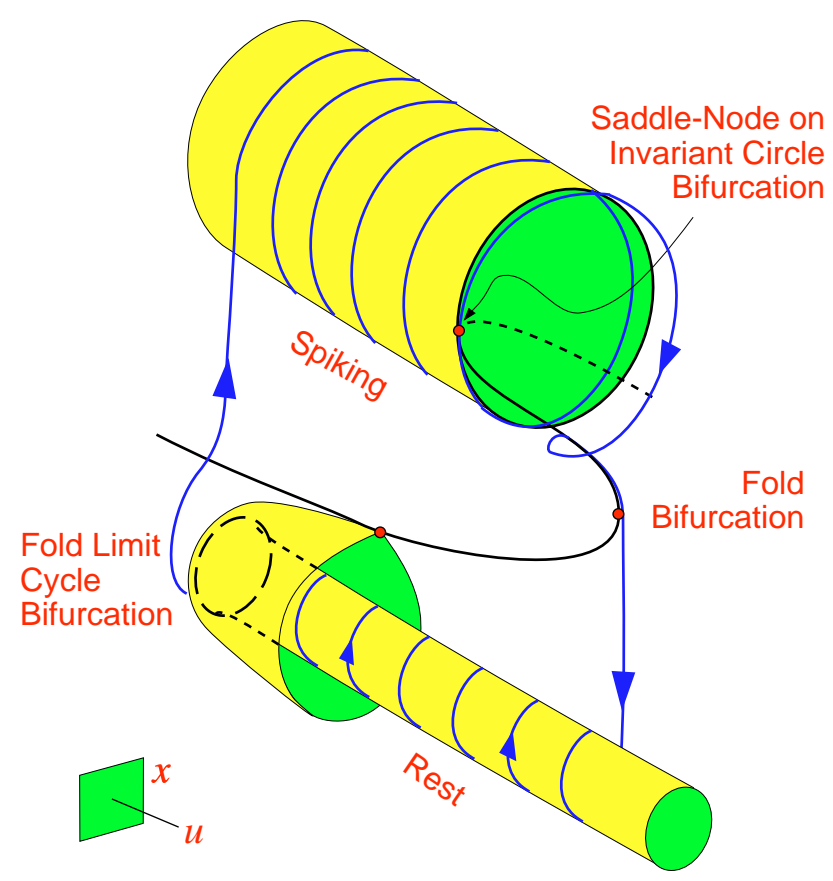

Fig. 98. "Fold cycle/circle" bursting via "fold cycle/fold" hysteresis loop: The quiescent state disappears via fold limit cycle bifurcation and the spiking state disappears via saddlenode on invariant circle bifurcation.

to a point via supercritical Andronov-Hopf bifurcation; see Fig. 99.

\subsection{Intermediate $\mu$}

The basic idea behind the dissection of the fastslow bursting is that the relaxation parameter $\mu$ in (21) is as small as we wish so that the slow variable $u$ may be assumed to be static on the fast time scale. In practice, $\mu$ may not be infinitesimal, but may assume intermediate values; e.g. $\mu=0.1$ in 


\section{"Fold Cycle/Hopf" Bursting}

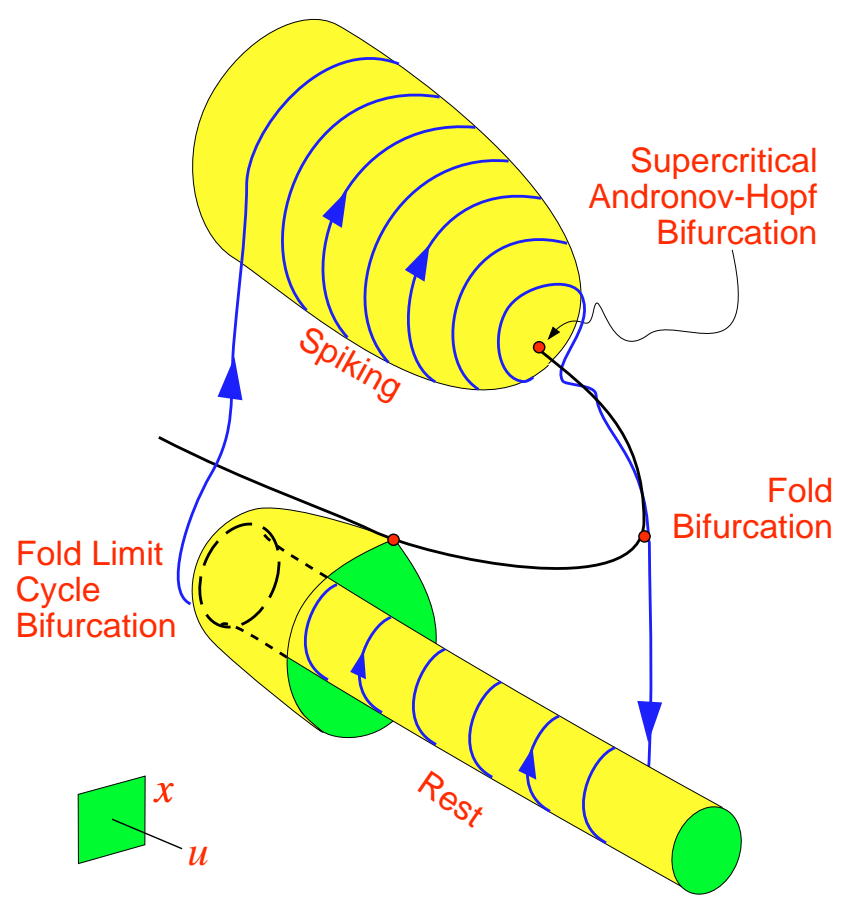

Fig. 99. "Fold cycle/Hopf" bursting via "fold cycle/fold" hysteresis loop: The quiescent state disappears via fold limit cycle bifurcation and the spiking state disappears via supercritical Andronov-Hopf bifurcation.

Figs. 68 and 79 and $\mu=0.01$ in many other illustrations. The theory developed above is valid for intermediate $\mu$ as well. However, one should be careful, since many counterintuitive phenomena can occur when $\mu$ is not "sufficiently" small (how small is "sufficient" depends on details of the model under consideration). In particular, a fast-intermediately slow system can exhibit bursting behavior even when the fast subsystem does not have periodic attractor for any value of the slow variable. There are two mechanisms that could lead to such a bizarre behavior. We consider them below.

\subsubsection{Modulated excitability}

Consider a system having nullclines as in the top left corner of Fig. 100. Such a system has a unique attractor - a stable equilibrium, and the solution always converges to it. Suppose the system depends on a slow variable $I$ which controls the vertical position of the $N$-shaped nullcline. If $I$ increases, the nullcline moves up slowly, and so does the solution because it tracks the equilibrium. However, if the increasing rate is not slow enough, the so- lution starts to oscillate with a large amplitude. Thus, the system exhibits spiking behavior even though it does not have a limit cycle attractor for any fixed $I$. Similarly, a system having a unique globally asymptotically stable attractor - a limit cycle (see top right corner of Fig. 100) may exhibit low amplitude quiescent behavior when the nullcline moves down.

In all these cases studying the behavior of the fast subsystem for fixed values of the slow variable may not provide adequate information when the slow variable is allowed to evolve. One should consider the full fast-slow system.

Indeed, consider the case of equilibrium dynamics in Fig. 100. The globally asymptotically stable equilibrium is excitable for any fixed value of $I$. It has a periodic pseudo-orbit depicted in Fig. 101. Suppose $I$ is fixed or increases sufficiently slow. If a perturbation moves the solution to point $p$, then the solution runs along the orbit, exits through point $q$, and stays in a small neighborhood of the equilibrium until the next strong perturbation. This corresponds to a single spike. In contrast, if $I$ increases faster, then the exit point $q$ becomes the entry point $p$ by the time the solution completes the rotation. Thus, the spike starts again, and so on.

This dynamical mechanism is closely related to the mechanism of accommodation and anodal break excitation, and combined with appropriate dynamics of the slow variable $I$ it may lead to bursting behavior. Sivan et al. [1995] refer to this phenomenon as modulated excitability. Neurocomputational properties of such a burster would depend on the type of excitability of the equilibrium, which in turn depends on the bifurcation the equilibrium is near. Thus, one can still use the classification described above to classify such fastintermediately slow bursters.

\subsubsection{Point-point bursters}

Consider the four hysteresis loops depicted in Fig. 55. If the rate of attraction to the up-state is relatively weak in comparison with the magnitude of $\mu$, then the fast variable spends most of its time converging to the equilibrium. Whenever the convergence is of the form of damped oscillations, the system's behavior resembles bursting, as we illustrate in Figs. 102 and 103 using the "fold/fold" and "fold/subHopf" hysteresis loops. We refer to such bursters as being point-point bursters. The remaining "subHopf/fold" and "subHopf/subHopf" 


\section{Equilibrium Dynamics}
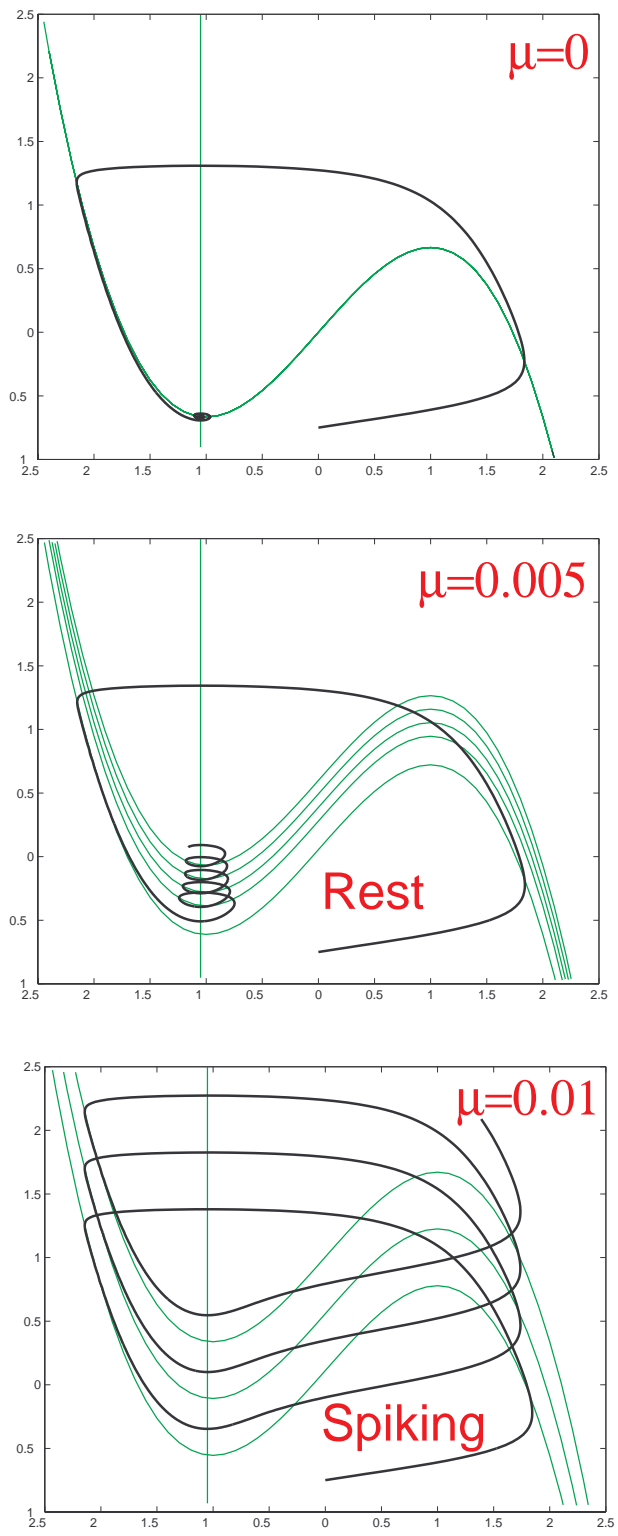

Periodic Dynamics
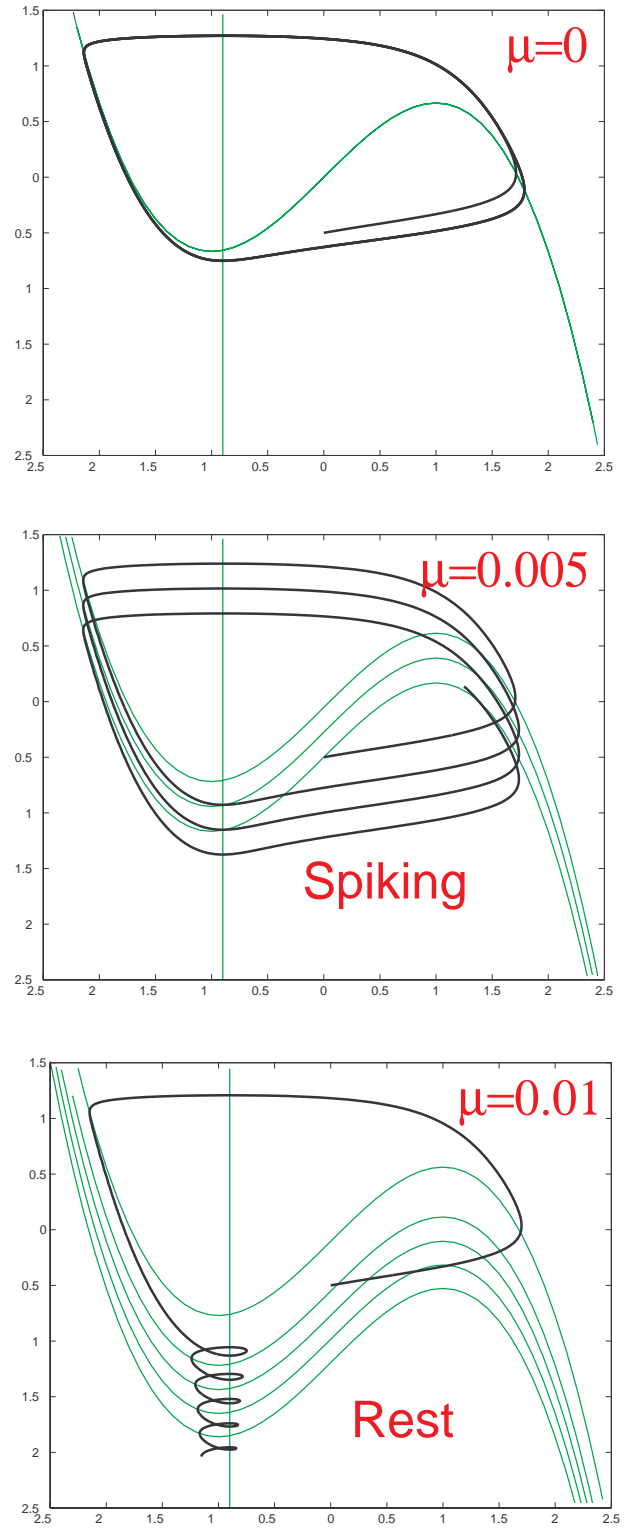

Fig. 100. Left: The system has a unique attractor — equilibrium, yet it can exhibit repetitive spiking activity when the $N$ shaped nullcline is moved up. Right: The system has a unique attractor - limit cycle, yet it can exhibit low amplitude quiescent activity when the $N$-shaped nullcline is moved down. Simulations of the Bonhoeffer-Van Der Pol oscillator $\dot{x}=I+x-x^{3} / 3-y$, $\dot{y}=\varepsilon(x+a)$ for $\varepsilon=0.1, a=1.05$ (left) or $a=0.9$ (right), and $\dot{I}=\mu$.

point-point bursters are illustrated elsewhere. It is remarkable that periodic bursting may occur despite the fact that the fast subsystem does not have a limit cycle attractor for any value of the slow variable.

It is believed that the "fold/subHopf" hysteresis loop point-point bursting is exhibited by the pituitary somatotroph cells, which secrete growth hormone [A. Sherman \& A. LeBeau, personal communication].

\subsection{Synchronization of bursters}

In this section we consider a weakly connected system of the form

$$
\begin{aligned}
& \dot{x}_{i}=f_{i}\left(x_{i}, u_{i}\right)+\varepsilon p_{i}(x, u, \varepsilon) \\
& \dot{u}_{i}=\mu\left[g_{i}\left(x_{i}, u_{i}\right)+\varepsilon q_{i}(x, u, \varepsilon)\right]
\end{aligned}
$$

where $x=\left(x_{1}, \ldots, x_{n}\right) \in \mathbb{R}^{m n}$ and $u=$ $\left(u_{1}, \ldots, u_{n}\right) \in \mathbb{R}^{k n}$ are vectors of the fast and slow 


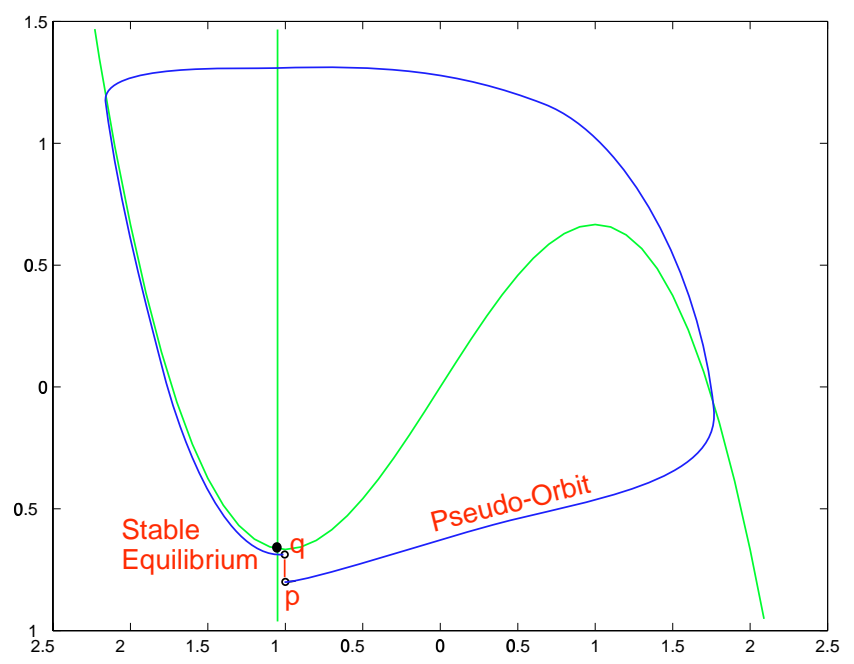

Fig. 101. A periodic pseudo-orbit in Bonhoeffer-Van Der Pol oscillator from Fig. 100 having (excitable) asymptotically stable equilibrium; i.e. a piece of solution starting from a point $p$ and exiting at a nearby point $q$.

variables, respectively, and $\varepsilon \ll 1$ is the strength of connections. We assume that each subsystem

$$
\begin{aligned}
& \dot{x}_{i}=f_{i}\left(x_{i}, u_{i}\right) \\
& \dot{u}_{i}=\mu g_{i}\left(x_{i}, u_{i}\right)
\end{aligned}
$$

exhibits periodic hysteresis loop bursting behavior. We also assume that the coupling is relatively strong in comparison with the magnitude of $\mu$; that is,

$$
\mu=\mathcal{O}(\varepsilon),
$$

which also includes the case $\mu \ll \varepsilon$. The case of extremely weak coupling $(\mu \gg \varepsilon)$ is not interesting for our discussion here.

There are two rhythmic processes associated with each burster: repetitive spiking and repetitive bursting. Therefore, there could be at least two different regimes of synchronization, which are depicted in Fig. 104:

- Synchronization of individual spikes.

- Synchronization of bursts.

One of them does not imply the other. Of course, there is an additional regime when both types of synchronization occur simultaneously.

\subsubsection{Spike synchronization}

A naive approach to study spike synchronization is to consider the fast subsystem (30), which is a weakly connected network of limit cycle oscillators. In Sec. 3.5 we show that the oscillators cannot lock unless they have nearly equal or loworder resonant frequencies. Thus, synchronization of individual spikes within a single burst depends crucially on the interspike frequencies. The frequencies, however, may vary substantially during the burst, because they depend on the values of the slow variables $u_{1}, \ldots, u_{n}$. A rigorous averaging theory for such systems is quite complicated [Arnold, 1982].

Spike synchronization may occur during the entire duration of a burst, or during the initial or the final stage of the burst. We present a nonrigorous discussion of these cases below.

\subsubsection{Initial stage}

Spike synchronization during the initial stage of the burst usually does not occur between "circle/*" bursters (where "*" means a wildcard) because of the following two reasons: (i) The interspike frequency changes substantially during the initial stage. Hence, small deviations in the values of the slow variables may lead to drastic variances in the frequencies. (ii) The canonical model for Class 1 systems (11) does not exhibit stable locking. Similar arguments can be applied to the "blue-sky/*" and "torus/*" bursters.

The other types of bursters tend to have regular spiking during the initial stage of a burst. Therefore, they could exhibit spike synchronization. However, the convergence to the synchronized state takes as many as $\mathcal{O}(1 / \varepsilon)$ spikes, and can be longer than the entire initial stage.

\subsubsection{Final stage}

Using nonrigorous arguments similar to those presented above, one can conclude that "*/circle", "*/blue-sky", and "*/torus" bursters do not usually exhibit spike synchronization during the final stage of the burst.

The case of "*/* homoclinic" bursting is subtler. The interspike frequency decreases as the function $1 /|\ln \lambda|$ (see Table 2), which does not seem to vanish unless the distance to the bifurcation, $\lambda$, is very small. Hence, the sudden drop in the interspike frequency occurs during the last few spikes, which may lead to their desynchronization, as one can see in Fig. 106. 


\section{"Fold/Fold" Bursting}
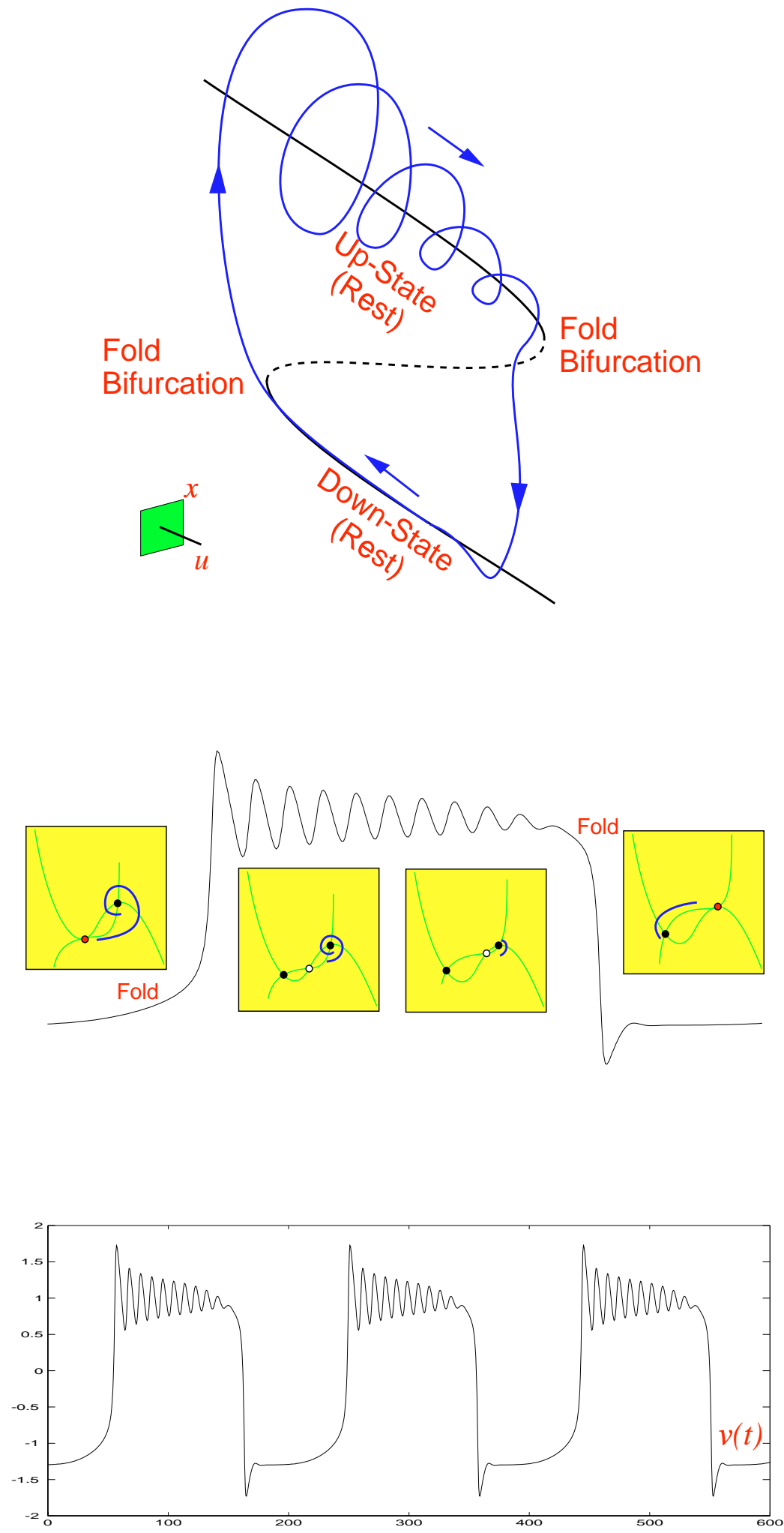

Fig. 102. "Fold/fold" hysteresis loop bursting of point-point type. Notice that the fast subsystem does not have a limit cycle attractor for any value of the slow variable. Bursting occurs because the rate of convergence to the up-state is relatively weak. Shown are simulations of the model (15) with parameters of the slow subsystem $\dot{c}=\mu v$. Parameters: $(a, b, d, \varepsilon, \mu)=(-1.3$, $2.3,0.1,0.5,0.01)$. 


\section{"Fold/SubHopf" Bursting}
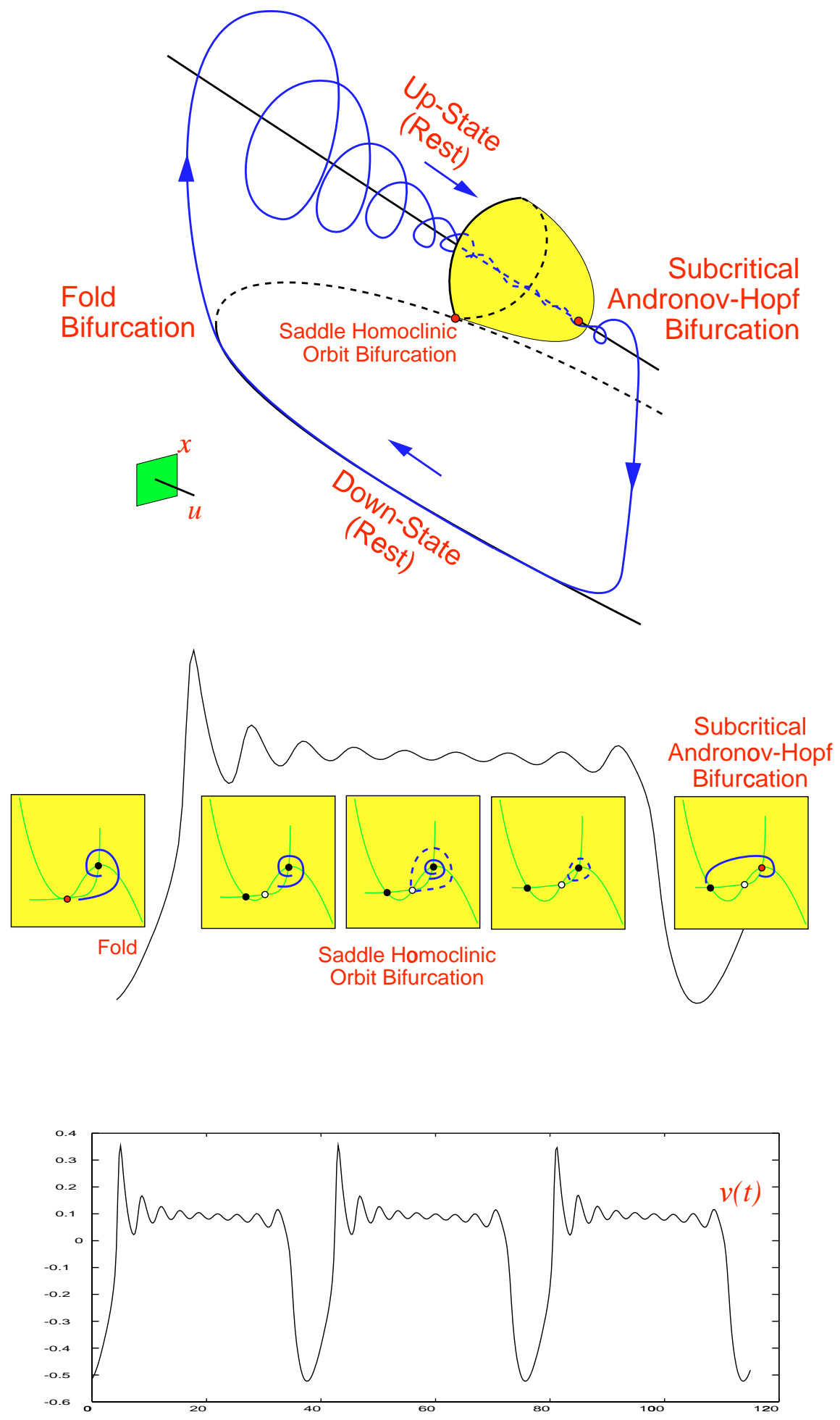

Fig. 103. "Fold/subHopf" hysteresis loop bursting of point-point type. The fast subsystem does not have a limit cycle attractor for any value of the slow variable. Bursting occurs because the rate of convergence to the up-state is relatively weak. Shown are simulations of the Morris-Lecar model (25) with $I(u)=-u$ and the slow subsystem $\dot{u}=\mu V$. Parameters: $V_{3}=0.107, V_{4}=0.07, g_{\mathrm{Ca}}=1.33, \mu=1 / 8$, the rest as in Fig. 59 . 


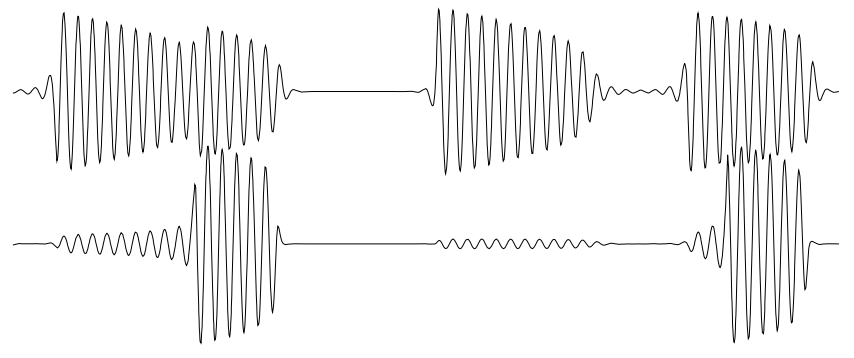

Spike Synchronization

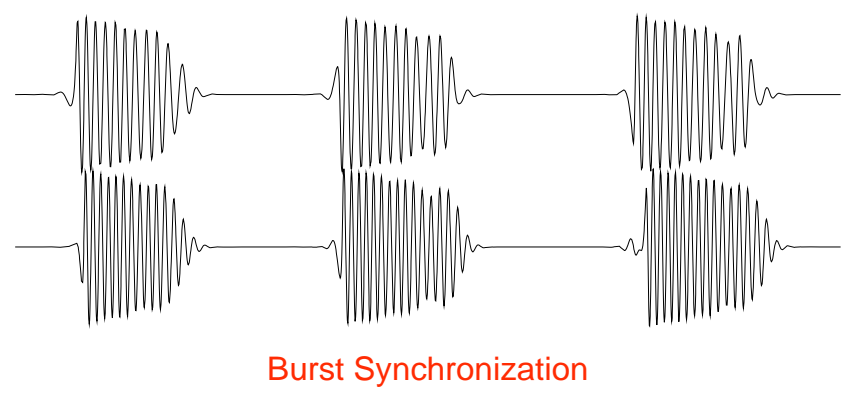

Fig. 104. Various regimes of synchronization of bursters (modified from [Izhikevich, 2000a]).

\subsubsection{Burst synchronization}

Dynamics of burst synchronization of weakly coupled bursters is similar in some aspects to that of strongly coupled relaxation oscillators.

To understand the mechanism of stable burst synchronization we consider two planar point-cycle bursters: One in the active (spiking) state, and the other still in the quiescent down-state. If the active burster makes the quiescent burster jump from the down-state prematurely, then they may exhibit stable burst synchronization. If it prolongs the quiescent down-state, then the in-phase burst synchronization cannot be stable, but some other regime, such as anti-phase burst synchronization, may occur.

We distinguish two mechanisms that lead to stable burst synchronization depending on whether the quiescent burster is an integrator or a resonator.

\subsubsection{Integrators}

If point-cycle bursting occurs via "fold/*" hysteresis loop, then the burster acts as an integrator. While the slow variable is near the bifurcation value, the fast variable is ready to jump up in response to incoming pulses from the active burster. This jump may lead to repetitive spiking when the

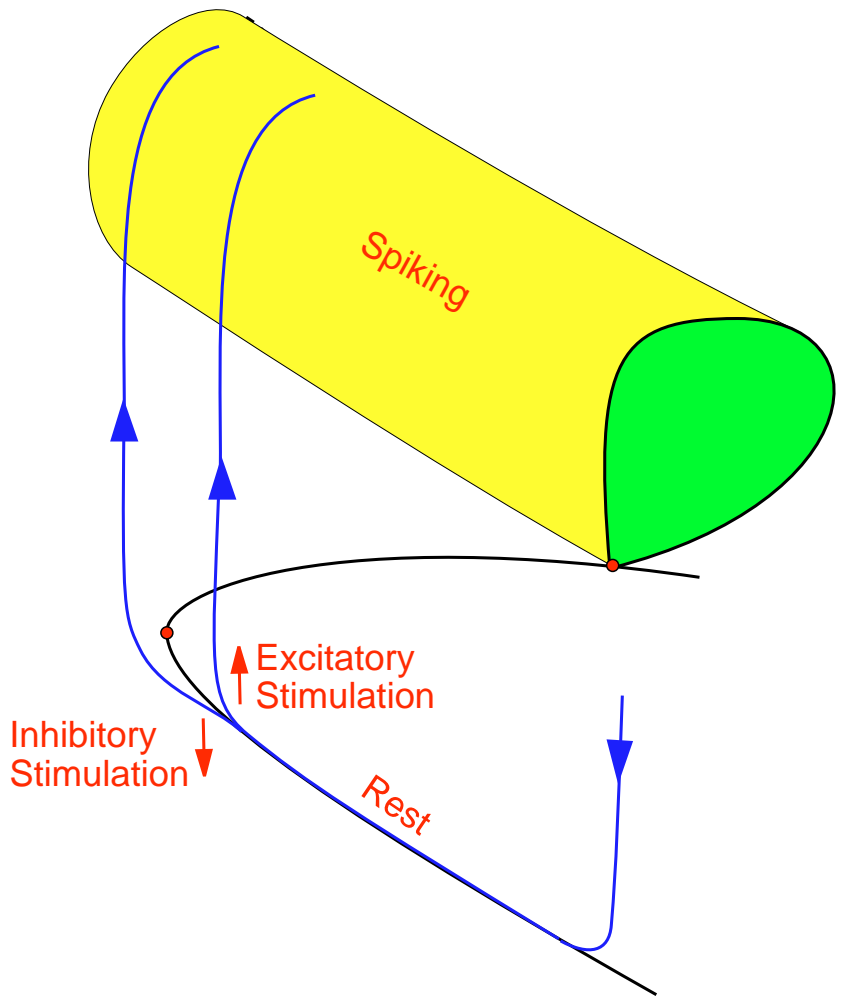

Fig. 105. Excitatory (inhibitory) pulses shorten (lengthen) the quiescent state in "fold/*" hysteresis loop bursters. This may lead to burst synchronization (desynchronization).

bursting is of "fold/*" type, or to the up-state when the bursting is of "circle/*" or "Hopf/*" type. In any case the jump shortens the quiescent phase.

If the synaptic connections are excitable, the quiescent burster does jump prematurely. The higher the frequency of the incoming pulses, the sooner it jumps. In contrast, if the connections are inhibitory, the quiescent down-state is prolonged; see Fig. 105.

Shortening the quiescent state is necessary but not sufficient condition for stable synchronization. The latter has yet to be found; It will depend on the bifurcation of the spiking state, and probably be similar to the compression hypothesis in the Fast Threshold Modulation (FTM) theory of synchronization of strongly connected relaxation oscillators [Somers \& Kopell, 1993, 1995]: The rate of change of slow variable before the jump is less than that after the jump. In this case the bursters may behave according to a simple rule excitation means synchronization, inhibition means desynchronization, as we illustrate in Fig. 106. 

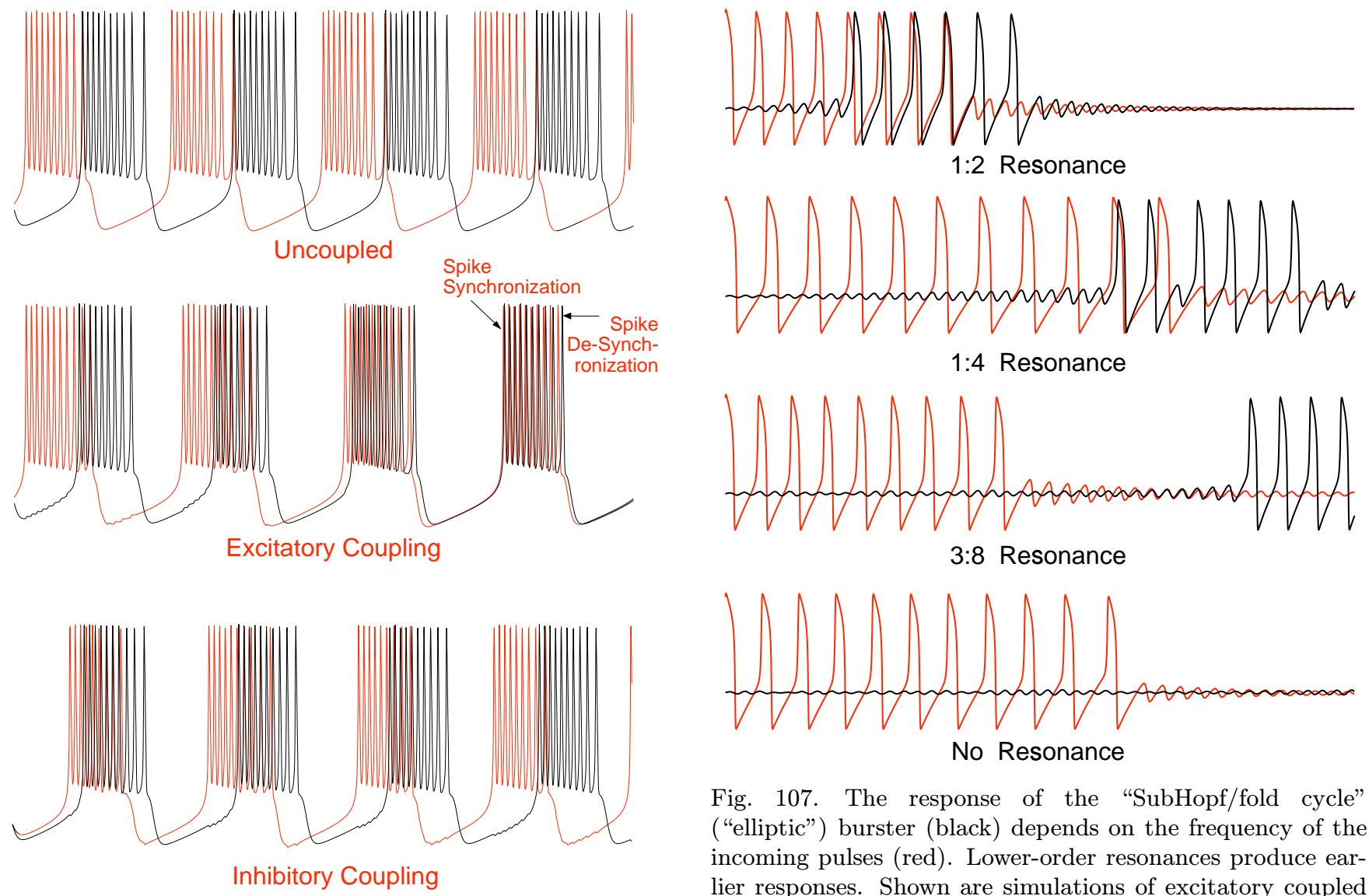

Fig. 107. The response of the "SubHopf/fold cycle" ("elliptic") burster (black) depends on the frequency of the incoming pulses (red). Lower-order resonances produce earlier responses. Shown are simulations of excitatory coupled FitzHugh-Rinzel bursters [Izhikevich, 2000a].

Fig. 106. Burst synchronization and desynchronization in "fold/homoclinic" ("square-wave") bursters. Shown are simulations of coupled Morris-Lecar systems with additional slow variables.

\subsubsection{Resonators}

If point-cycle bursting occurs via "subHopf/*" hysteresis loop, then the burster acts as a resonator in the quiescent down-state. Such a burster exhibits damped subthreshold oscillations of the membrane potential, see Sec. 2.2. If the frequency of the incoming pulses is resonant with the frequency of the subthreshold oscillation, then the quiescent burster jumps up prematurely, which may result in stable in-phase burst synchronization. The lower the order of resonance is, the sooner it jumps, as we illustrate in Fig. 107. If the input pulse train is not resonant, the quiescent down-state is not affected.

A seemingly counterintuitive observation is that the sign of synapse does not affect burst synchronization, but may only affect spike synchronization, as we illustrate in Fig. 108. This observation agrees with the fact that a resonator can fire in response to excitatory as well as inhibitory pulse, as we discuss in Sec. 2.2. Therefore, in contrast to

the previously discussed case, both excitation and inhibition can lead to burst synchronization.

\subsubsection{Delayed onset of bursting}

There is an interesting phenomenon that is ubiquitous in computer simulations of bursters but may never be encountered in experiments: The onset of bursting can be delayed significantly when the variable $u$ passes slowly a bifurcation value. That is, the fast variable remains quiescent despite the fact that the slow variable has already crossed the bifurcation value and the quiescent state has already become unstable or disappeared. Because such a quiescence is highly unstable, small perturbations from the other bursters can cause an immediate transition to the active state, which may result in instantaneous synchronization.

There are two different but related mechanisms for such a delay.

\subsubsection{Slow passage effect}

First, consider the case when the quiescent state loses stability, e.g. via an Andronov-Hopf bifurca- 

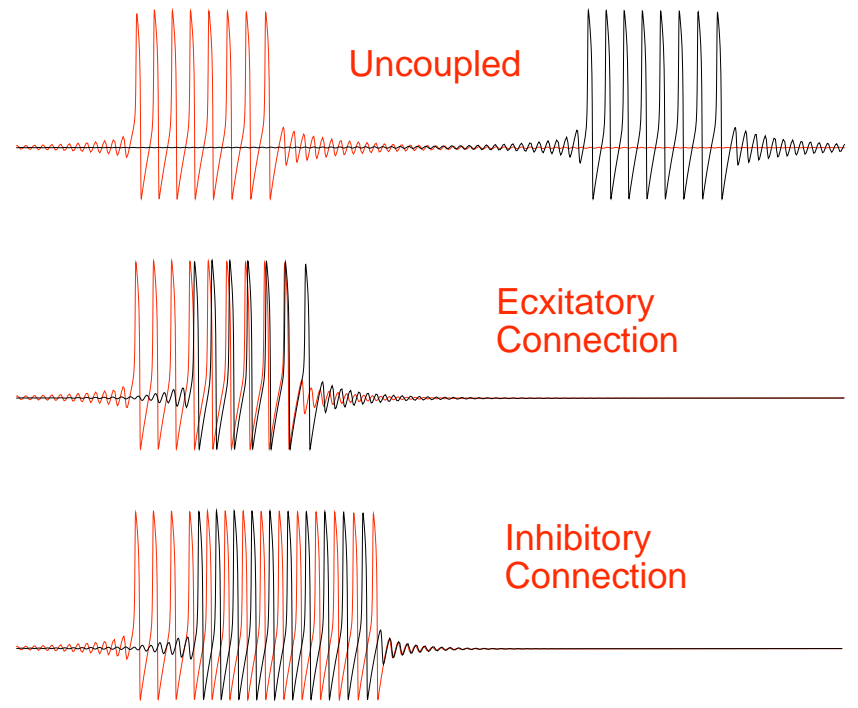

Fig. 108. The type of synaptic connection does not affect burst synchronization in coupled "SubHopf/fold cycle" ("elliptic") bursters. It affects only the spike synchronization, which is in-phase for excitatory synapses and anti-phase for inhibitory synapses. Shown are simulations FitzHugh-Rinzel bursters [Izhikevich, 2000a].
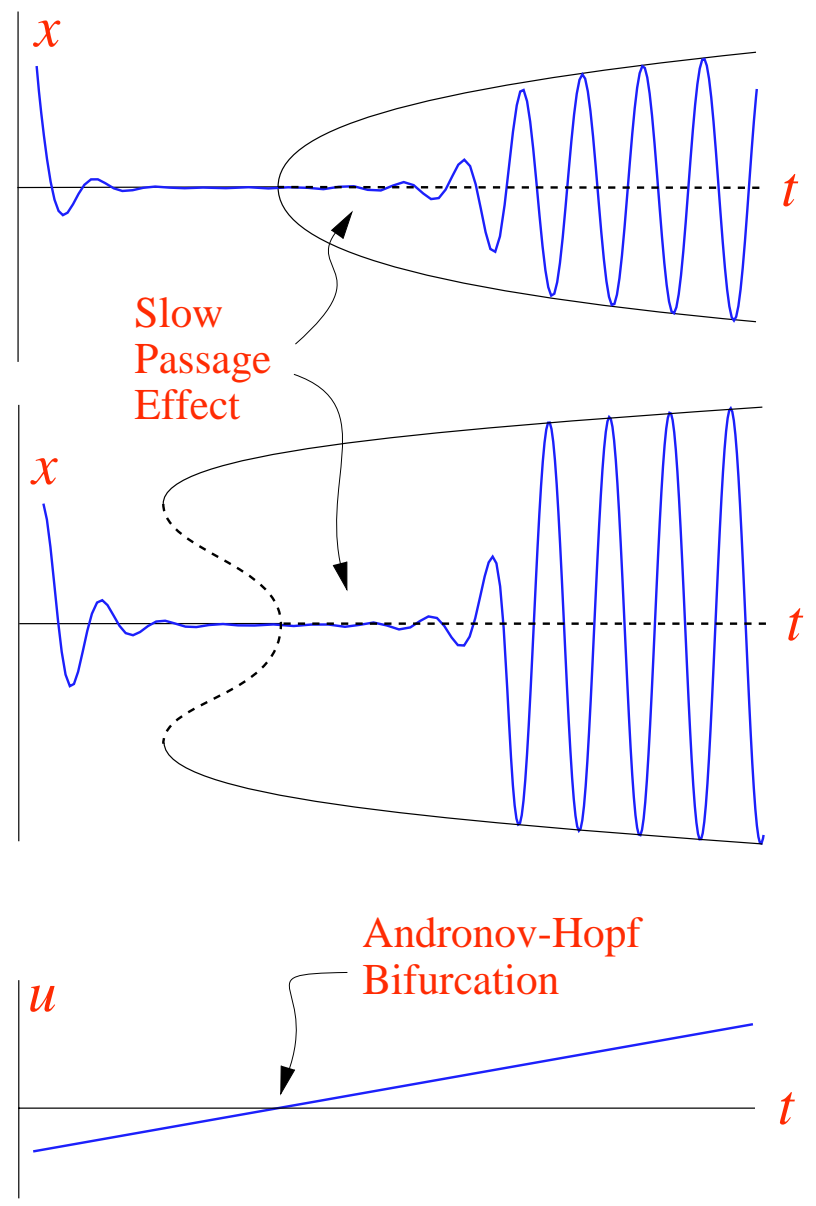

Fig. 109. Slow passage through Andronov-Hopf bifurcation. (Modified from [Hoppensteadt \& Izhikevich, 1997].) tion. When the rest state becomes unstable, the fast variable still needs some time to diverge from the equilibrium, which can be significant, as we illustrate in Fig. 109. We encounter this phenomenon when we consider "Hopf/Hopf" and "SubHopf/fold cycle" bursting. The slow passage effect can also be observed near Neimark-Sacker bifurcation.

The slow passage effect is very sensitive to whether or not the system is analytical [Nejshtadt, 1985]. Many mathematical models of bursters are analytical, and hence they exhibit such an effect in computer simulations. However, the effect can be a mathematical artifact, since it has yet to be seen in experiments.

The slow passage effect can be shortened significantly by noise or weak input from other bursters. The latter provides a powerful mechanism for instantaneous synchronization of bursters even when they have essentially different interburst frequencies. For example, a pair of "SubHopf/fold cycle" bursters from Fig. 79 can synchronize inphase instantaneously (if coupled) because the slower burster spends half of the quiescent time near the unstable equilibrium and is ready to fire in response to the very first pulse coming from the faster burster.

\subsubsection{French duck (canard) solutions}

When a relaxation system has nullclines intersected as in Fig. 110, it may have a so-called French duck (canard) periodic solution [Eckhaus, 1983]. After the fast variable reached the left knee point, it does not jump to the right branch, but stays near the unstable middle branch for some time. Similar phenomenon may occur in "fold/*", "fold cycle/*", and "*homoclinic/*" bursters [Booth et al., 1997], as we illustrate in Fig. 111. The fast variable stays near the unstable state for some time before jumping to the limit cycle attractor. A necessary condition for such a delayed quiescence is that the slow subsystem has an equilibrium near the bifurcation value. That is, the manifold of equilibria of the slow subsystem depicted as a yellow plane in Fig. 111 should pass near the left knee point.

The fast variable near the unstable branch is highly susceptible to small perturbations, e.g. those coming from the other bursters. An excitable spike can make it fire, resulting in instantaneous synchronization. In contrast, an inhibitory spike can make it jump to the lower (stable) branch, thereby delaying the onset of bursting even further. 


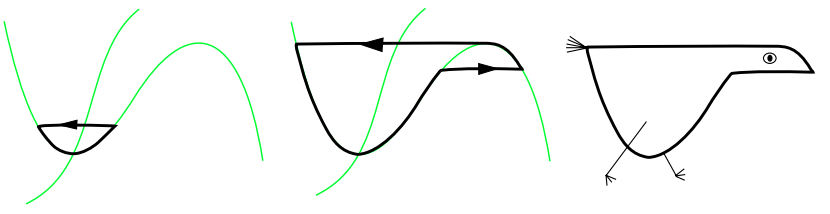

Fig. 110. French duck (canard) solutions in relaxation systems.

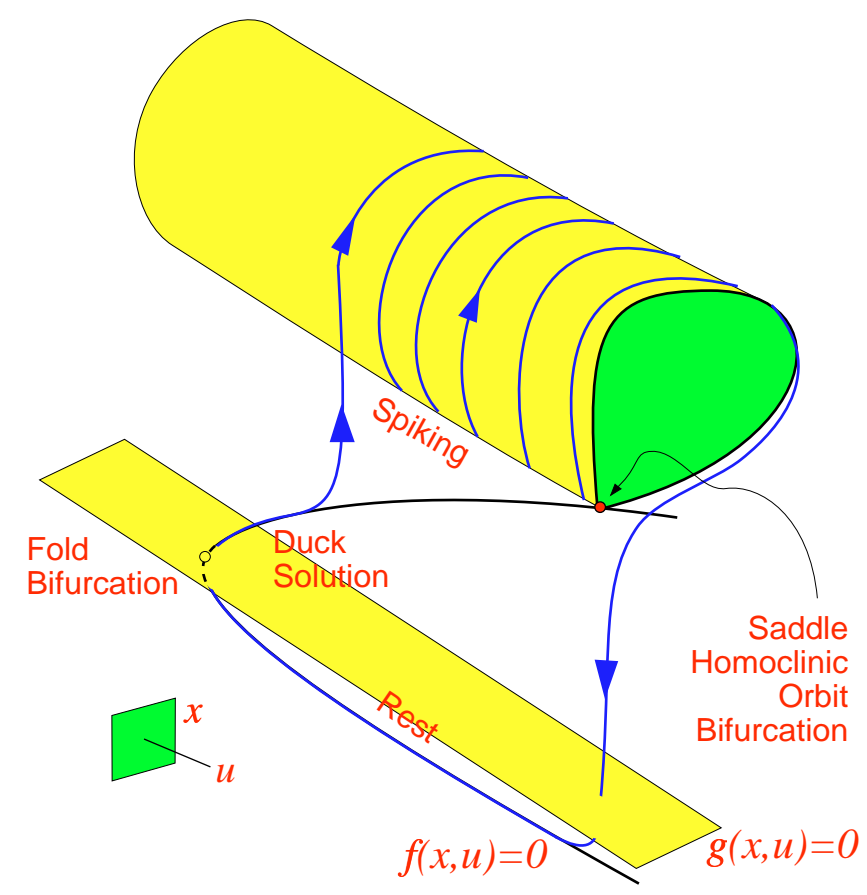

Fig. 111. The onset of bursting is delayed in the "fold/homoclinic" ("square-wave") burster due to the French duck (canard) phenomenon.

Since French duck solution exists in a narrow range of parameters [Arnold et al., 1994], and it is sensitive to noise, it is not clear whether the phenomenon of delayed onset of bursting can occur in real cells.

Similarly, we do not discuss here the phenomenon of delayed transition to the quiescent state, which occurs in "*/homoclinic" bursters as we illustrate in Fig. 112. A tiny transition delay can be seen in almost all simulations of "*/homoclinic" bursters presented in this paper. A significant delay may even affect synchronization properties of bursters in computer simulations, but it is also unlikely to be encountered in real cells.

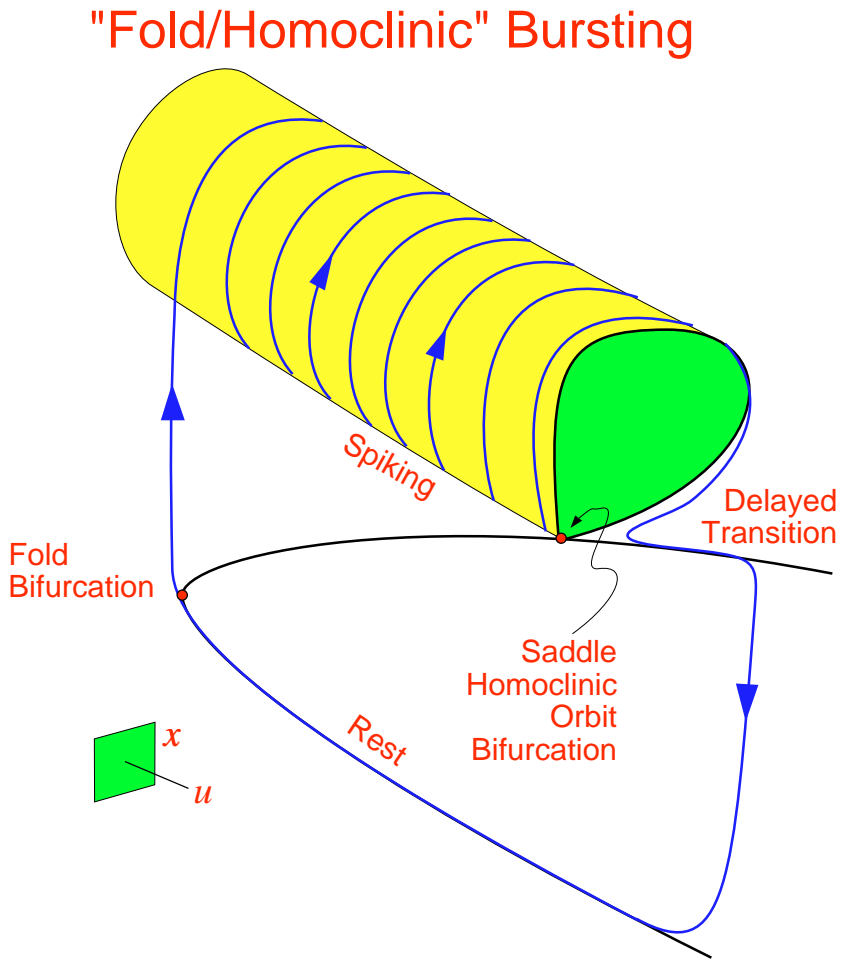

Fig. 112. Delayed transition to the quiescent state during "fold/homoclinic" bursting.

\subsubsection{Rate of locking}

The rate of convergence to attractors in weakly connected networks is very slow; namely, it is of order $\varepsilon \ll 1$, where $\varepsilon$ is the strength of connections [Hoppensteadt \& Izhikevich, 1997]. For example, a weakly connected oscillatory network (18) needs as many as $\mathcal{O}(1 / \varepsilon)$ spikes to synchronize ${ }^{2}$ regardless of whether each element is a smooth or a relaxation oscillator. This is the major source of criticism of weakly connected models, since the slow rate of convergence contradicts some experimental observations. For example, the CPG behavior in the lamprey is characterized by a strong rate of convergence to attractor (see e.g. [Kopell, 1995; Somers \& Kopell, 1995; Williams \& Sigvardt, 1995]), but it is modeled by a system of phase equations which is a canonical model for weakly connected oscillators.

Our analysis of weakly connected bursters suggests that the discrepancy in rates of convergence is not due to the assumption of weakness of connections, but due to the assumption that each segment

\footnotetext{
${ }^{2}$ Under synchronization we mean here convergence to a small but finite neighborhood of an appropriate attractor, which takes $\mathcal{O}(1 / \varepsilon)$ units of time. Complete convergence to the attractor is an asymptotic process that requires infinite amount of time, unless the system is non-Lipchitz or the initial state is on the attractor.
} 
of lamprey spinal cord can be modeled by an oscillator. If we model it by a burster, which it is, then the rate of convergence to an attractor looks "fast" compared to the interburst period despite the fact that the bursters are weakly connected. Indeed, it takes $\mathcal{O}(1 / \varepsilon)$ spikes to produce $\mathcal{O}(1)$ changes in the activity of a postsynaptic burster, but there could be as many as $\mathcal{O}(1 / \mu)$ spikes in each burst. If $\varepsilon$ and $\mu$ are of the same order, then one or two bursts are usually enough for locking. Another way to explain this is to note that locking of two bursters requires $\mathcal{O}(1 / \varepsilon)$ units of time, but each burst lasts $\mathcal{O}(1 / \mu)$ units. Therefore, locking may look instantaneous on the time scale of interburst intervals, especially when $\mu \ll \varepsilon$.

\section{Other Types}

In this section we briefly mention bursters that cannot be described by the fast-slow system (21). We distinguish three cases: Periodic, quasiperiodic, and chaotic bursting behavior.

\subsection{Periodic bursting: Hedgehog limit cycles}

Periodic bursting often arises when a system has hedgehog-like limit cycle attractor depicted in Fig. 113. We refer to such a burster as being hedgehog burster. Many fast-slow bursters considered in the previous section have periodic dynamics with the hedgehog attractor when $\mu$ is small, and they persist as hedgehog bursters when $\mu$ is intermediate or large.

Hedgehog bursting can be exhibited even by two-dimensional systems, e.g. those having twisted fast nullclines; see Fig. 114. Following are two hedgehog cases.

\subsubsection{Blue-sky catastrophe}

The hedgehog attractor can also arise when a neural system is near blue-sky catastrophe (Fig. 30) with the fold limit cycle bifurcation in the spiking area of the phase space. Indeed, the vector field is directed along the disappeared cycle, hence the solution spends most of its time rotating around it thereby firing spikes; see Fig. 115. The system exhibits periodic bursting behavior, although it cannot be decomposed into fast and slow subsystems of the form (21). Hence such bursting is not of the fast-slow type.

\subsubsection{Saddle-focus homoclinic orbit}

Now consider a neural system near a saddle-focus homoclinic orbit bifurcation; see top of Fig. 30. Under certain fairly general conditions found by Shilnikov (see [Kuznetsov, 1995]) the saddlefocus has secondary homoclinic orbits that can bifurcate (via secondary saddle-focus homoclinic bifurcations) into stable cycles corresponding to doublets, triplets, etc., see Fig. 116.

This is the mechanism of a generation of complex spikes in the FitzHugh-Nagumo partial differential equation [Hastings, 1976; Evans et al., 1982; Feroe, 1982], and it may be related to the mechanism of bursting in the LP cell of the lobster stomatogastric ganglion [Guckenheimer et al., 1997].

\subsection{Quasiperiodic bursting}

A continuous rhythmic signal $x(t)$ is quasiperiodic if there is a continuous function $q\left(\theta_{1}, \ldots, \theta_{k}\right)$, which is $2 \pi$-periodic in each argument, such that

$$
x(t)=q\left(\omega_{1} t, \ldots, \omega_{k} t\right), \quad \text { for all } t \geq 0,
$$

where $\Omega=\left(\omega_{1}, \ldots, \omega_{k}\right)^{\top} \in \mathbb{R}^{k}$ is a frequency vector [Samoilenko, 1991; Izhikevich, 1999a]. Quasiperiodic activity corresponds to a torus attractor, and it often looks like bursting or spindles waves; see Fig. 117. Its shape depends on the function $q$ and the frequency vector $\Omega$.

The torus attractor may appear, e.g. via supercritical Neimark-Sacker bifurcation. It can also appear via fold limit cycle bifurcation with a homoclinic torus structure (Fig. 30). When the cycle disappears, the system exhibits bursting behavior similar to the "blue-sky" bursting depicted in Fig. 115. Some additional cases are depicted in Fig. 118.

\subsection{Chaotic bursting}

Many chaotic signals resemble cycle-cycle bursting; see Fig. 119. Point-cycle bursting occurs, e.g. in the presence of Pomeau-Manneville intermittency. Many systems near saddle-focus homoclinic orbit bifurcation also exhibit point-cycle chaotic bursting [Kuznetsov, 1995] with a Rössler-like attractor; see Fig. 120.

On the other hand, many fast-slow bursters have chaotic dynamics. For example, the "fold/homoclinic" burster may have a horseshoe structure [Terman, 1991, 1992], and hence exhibit 


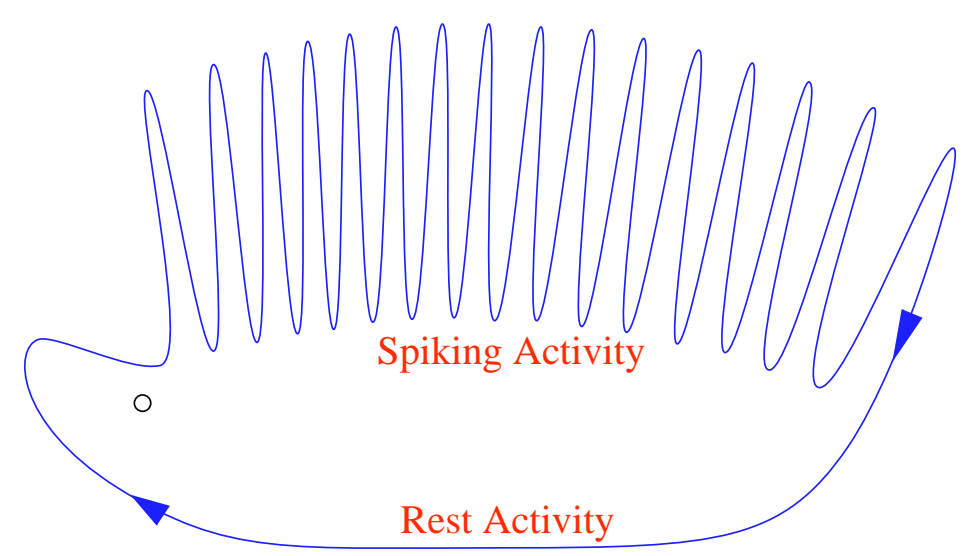

Fig. 113. A hedgehog-like limit cycle attractor often corresponds to bursting behavior (from [Hoppensteadt \& Izhikevich, 1997]).
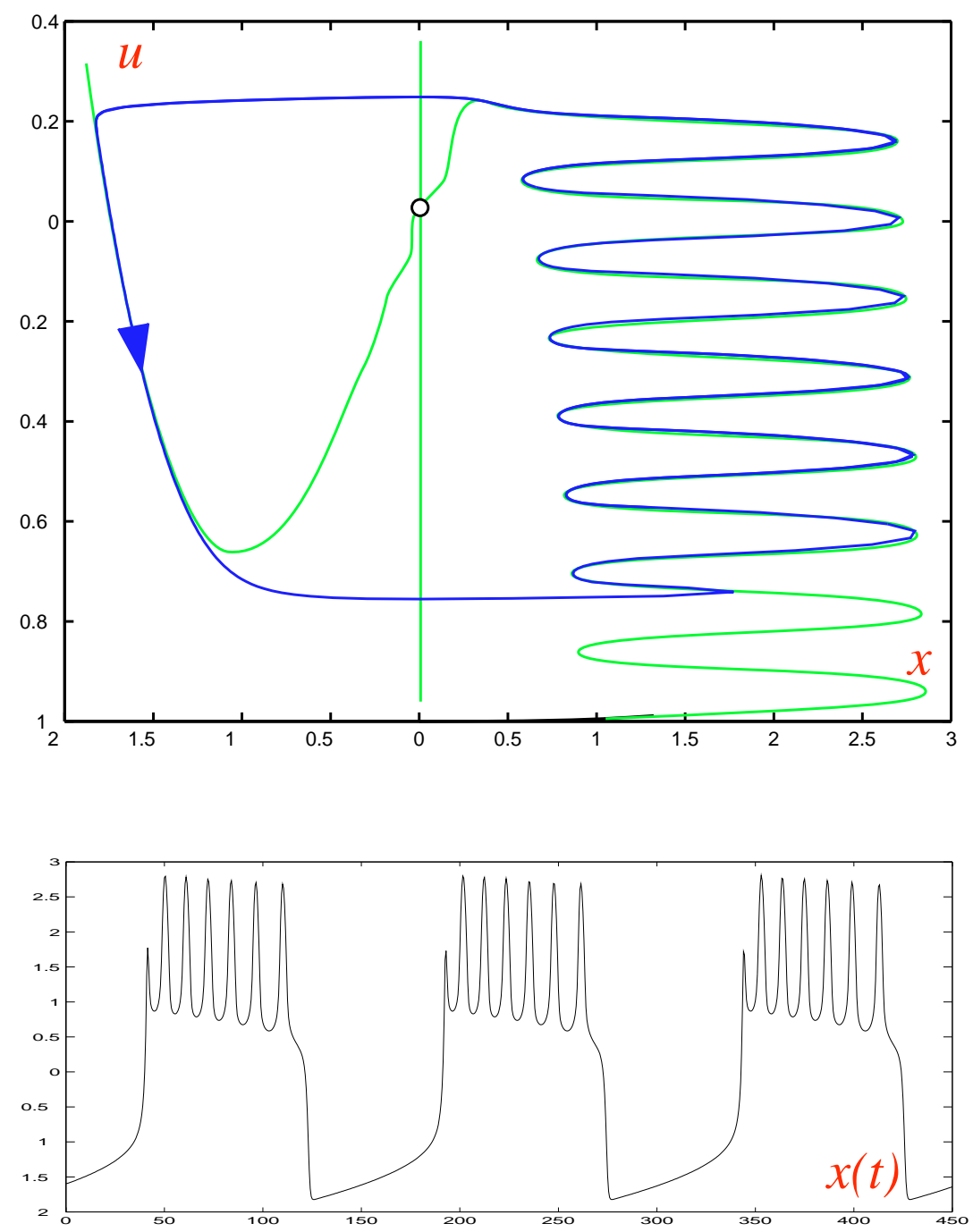

Fig. 114. Fast and slow nullclines (green) and Hedgehog limit cycle attractor (blue) in a planar relaxation system $\dot{x}=$ $x-x^{3} / 3-u+4 S(x) \cos 40 u, \dot{u}=\mu x$, where $S(x)=1 /\left(1+e^{5(1-x)}\right)$ and $\mu=0.01$. 

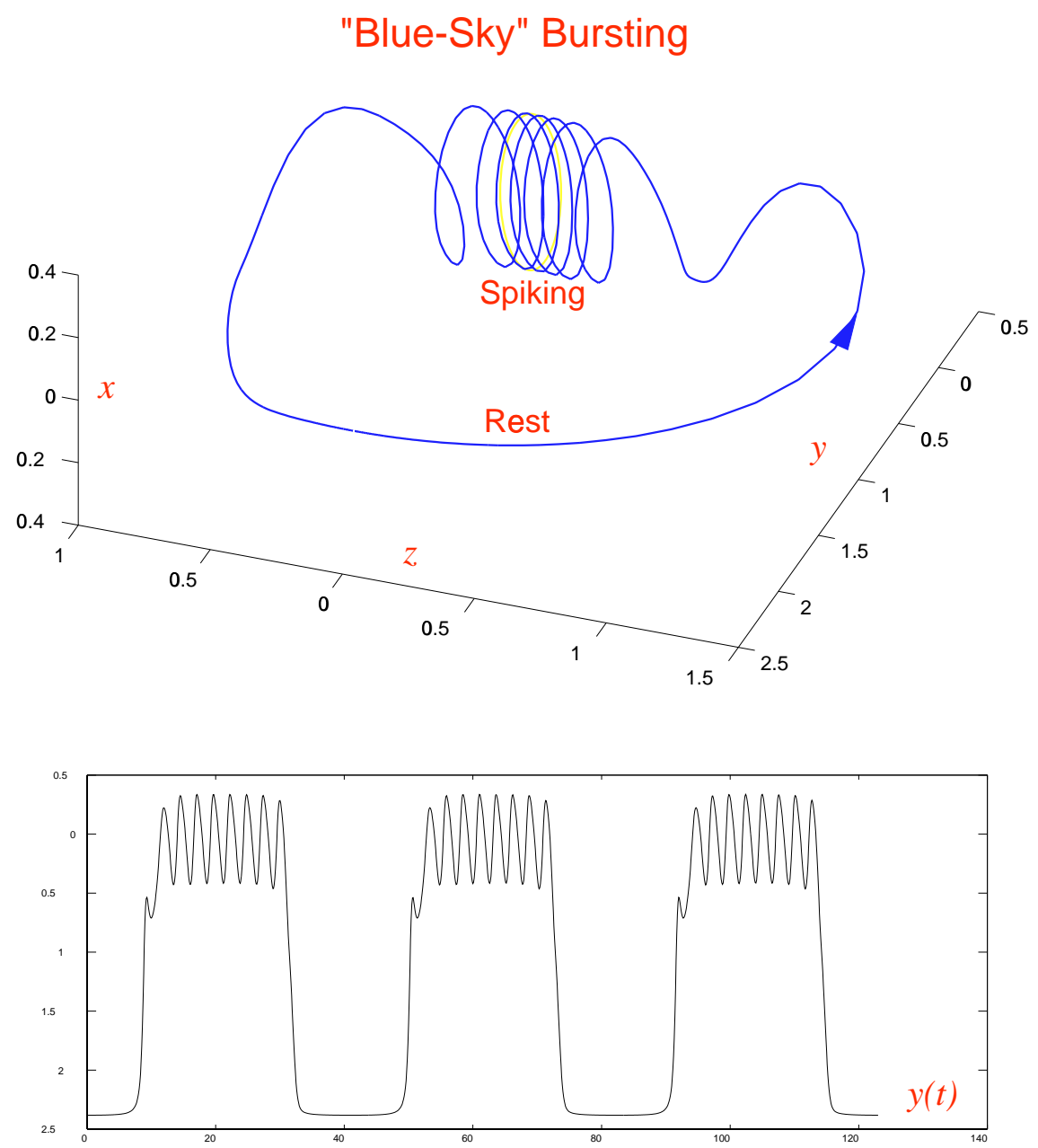

Fig. 115. Hedgehog bursting of the "Blue-sky" type. Simulations of the Gavrilov-Shilnikov model [Kuznetsov, 1995, p. 256]. Parameters: $b=10, \varepsilon=0.02, \mu=0.5$.
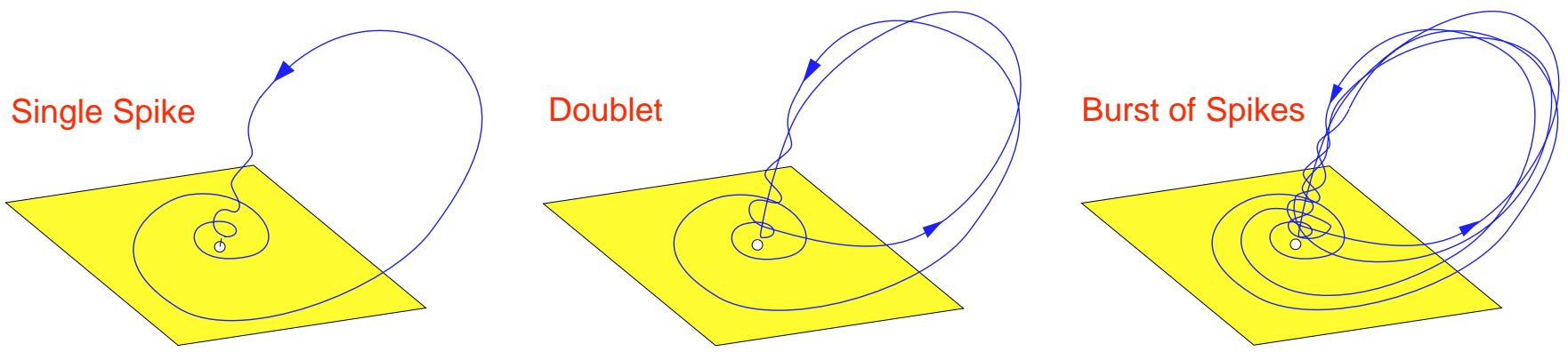

Fig. 116. Secondary homoclinic orbits to saddle-focus equilibrium can bifurcate into periodic solutions corresponding to doublets, triplets (not shown) or bursts of spikes. 


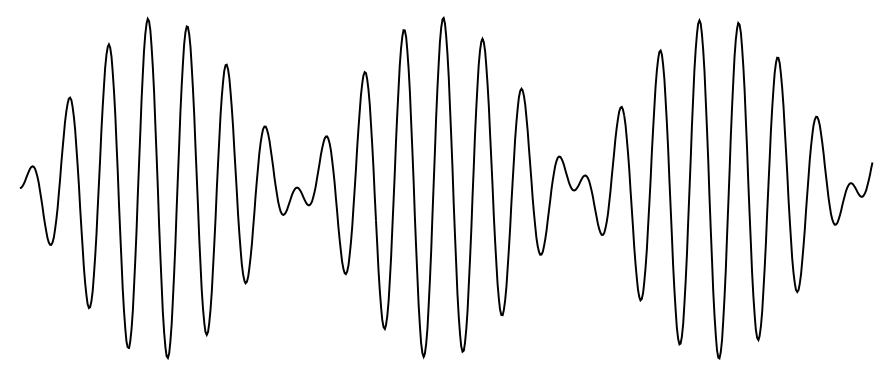

Fig. 117. An example of a quasiperiodic signal. $x(t)=\sin \omega_{1} t \sin \omega_{2} t$ with the frequency vector $\left(\omega_{1}, \omega_{2}\right)=(1, \sqrt{2} / 20)$.
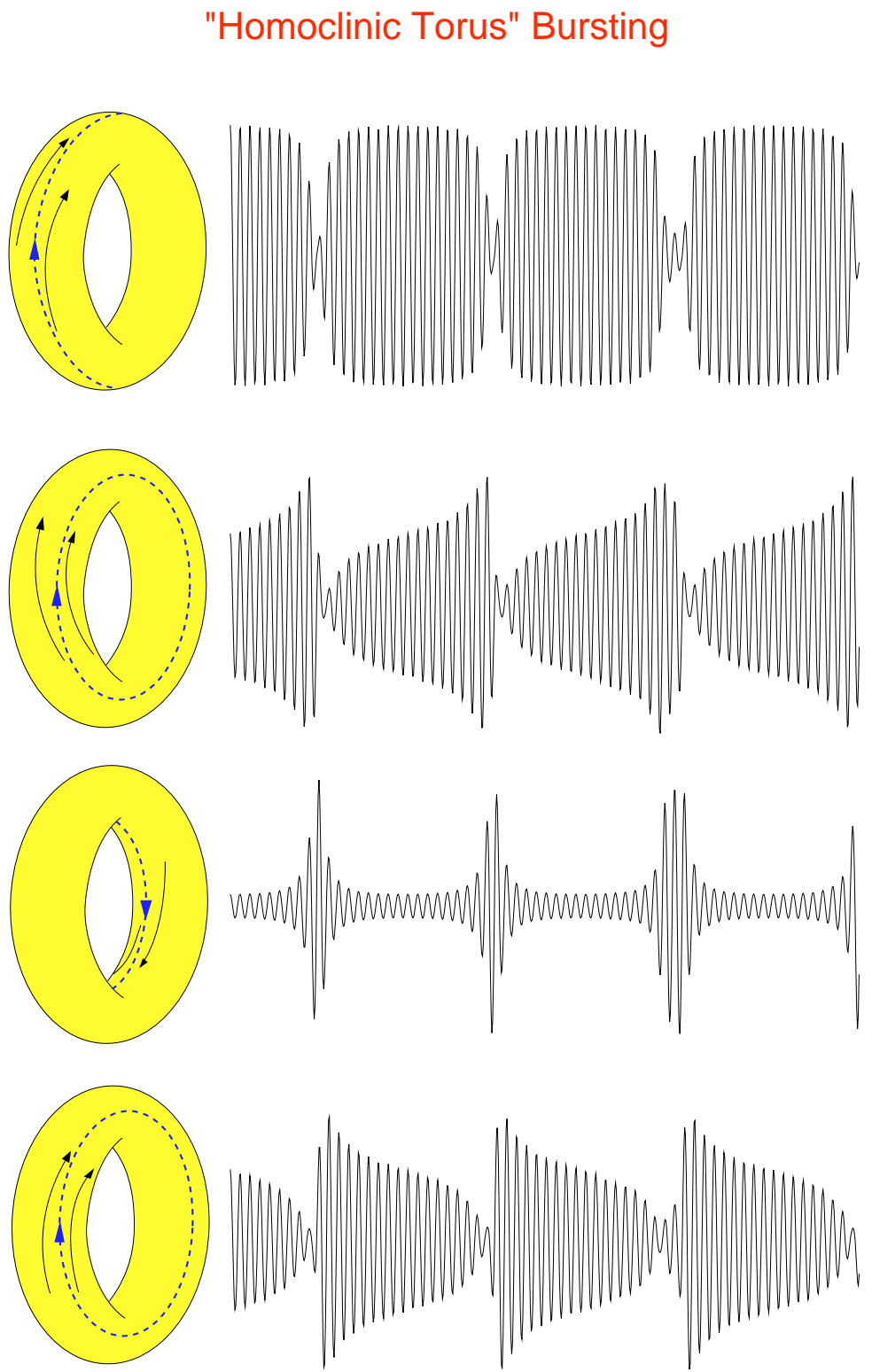

Fig. 118. "Homoclinic torus" bursting. The pattern depends on the location of the disappeared fold limit cycle (dotted blue circle). 


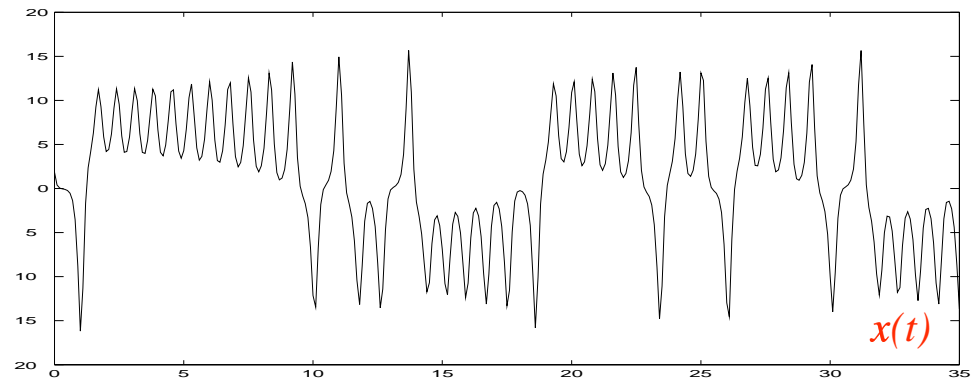

Fig. 119. Solution of the Lorenz system resembles chaotic cycle-cycle bursting.
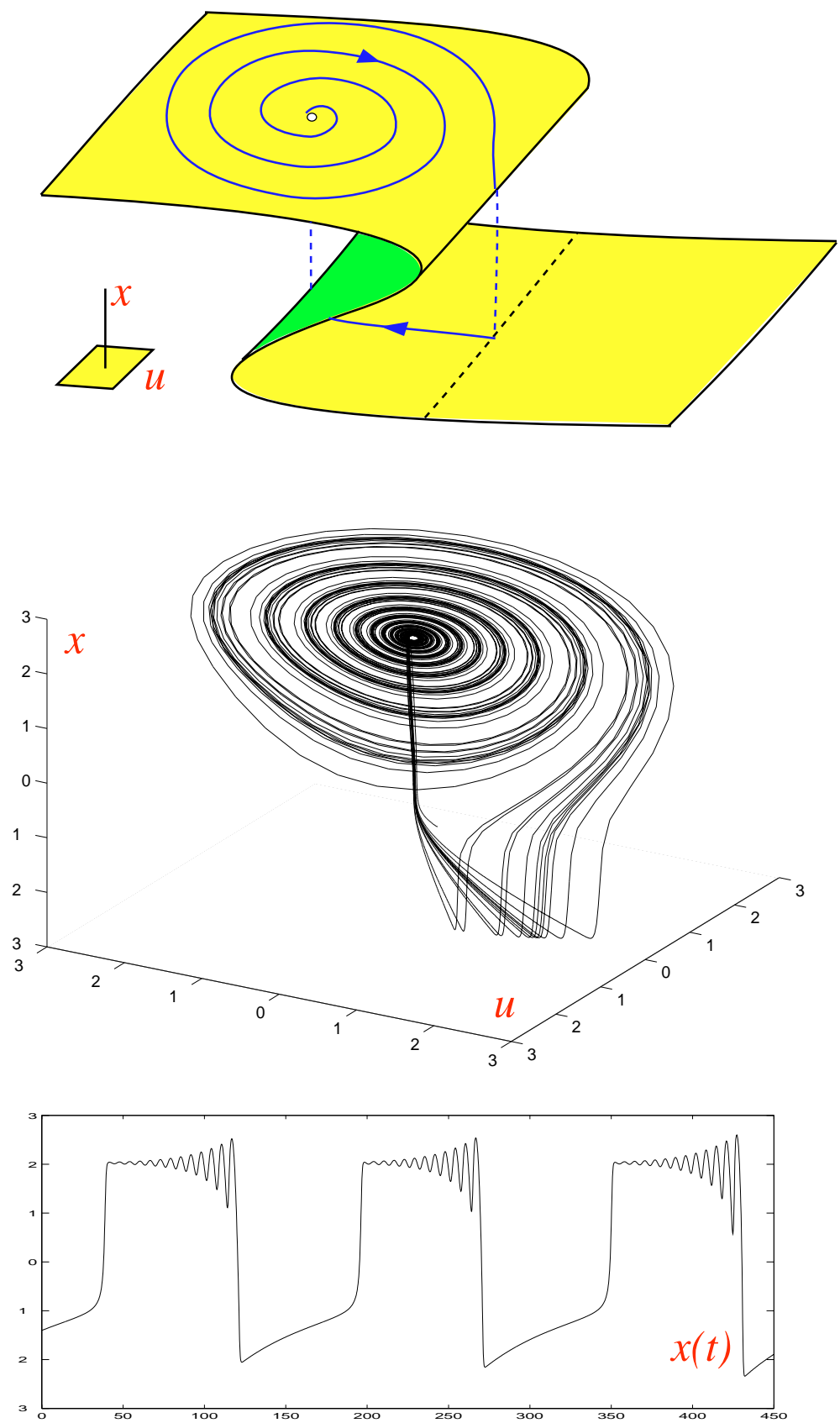

Fig. 120. Chaotic bursting near saddle-focus homoclinic orbit bifurcation. Simulations of a system $\dot{x}=x-x^{3} / 3+I-\operatorname{Re} u$, $\dot{u}=\mu(S(x)(1+\omega \mathrm{i}) u-u / 2)$, where $u \in \mathbb{C}, S(x)=1 /\left(1+e^{-5 x}\right), \omega=-10, I=0.75, \mu=0.1$. 
chaotic bursting. Terman's arguments can be generalized to most "*/homoclinic" bursters.

\section{Classification of Bursters: Why Bother?}

There are many types of distinct bursters from the purely dynamical system point of view. Are they really so different from the neuro-computational point of view? The answer is maybe.

Indeed, some bursters have quite unique neurocomputational properties. For example, the "circle/circle" ("parabolic") bursting exhibits Class 1 neural excitability and Class 1 spiking, and it may act as an integrator of neural signals when it occurs via "fold/*" hysteresis loop. In contrast, the "subHopf/fold cycle" ("elliptic") burster exhibits Class 2 neural excitability and Class 2 spiking, and it acts as a resonator. Interactions between such resonator bursters depend crucially on the resonant relations between their interspike frequencies [Izhikevich, 2000a]; they can exhibit spike synchronization, burst synchronization, or both. In contrast, "circle/circle" bursters do not have welldefined interspike frequencies, and hence are reluctant to exhibit spike synchronization.

Thus, different bursters can communicate, synchronize, and process information differently. However, we cannot exclude the case when a pair of distinct bursters has identical neuro-computational properties, since there are so many bursters [see Table 4], and so little information about their behavior.

\subsection{How to distinguish bursters?}

Since different bursters may have different neurocomputational properties, it is important to have criteria to distinguish them experimentally. The least useful, but probably the most common way, is to browse through this paper and try to find a picture that "resembles" micro-electrode recordings under consideration. This might be a complete waste of time since two identical bursters may look quite different (Fig. 121) or two quite distinct bursters may look "similar"; compare "circle/circle" bursting in Fig. 57 and "fold/big homoclinic" bursting in Fig. 86 .

\subsubsection{Frequency of emerging/ terminating spiking}

First, one should inspect the frequency of emerging and terminating spiking and compare it with

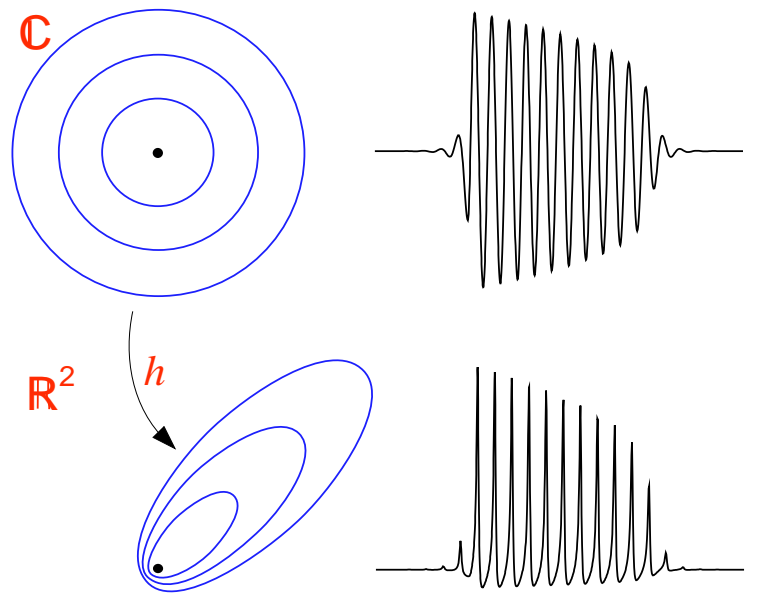

Fig. 121. Two topologically equivalent (via homeomorphism h) "subHopf/fold cycle" bursters may "look" quite different (from [Izhikevich, 2000a]).

the interspike frequency in the middle of a burst. A significant drop of the frequency is a good indicator of saddle-node on invariant circle and saddle homoclinic orbit bifurcations. For example, if we consider the planar case, then small initial frequency implies "circle/*" bursting. Similarly, small terminating frequency implies "*/circle" or "*/homoclinic" bursting. If there is no significant change in the frequency during the burst, then possible bifurcations are of fold or Andronov-Hopf type; see Tables 1 and 2 .

Guckenheimer et al. [1997] used heuristic arguments and obtained detailed asymptotics of the interspike frequency at the end of the burst, which could be used to distinguish, e.g. "*/circle" from "*/homoclinic" bursting.

\subsubsection{Amplitude of emerging/ terminating spiking}

A small amplitude initial or terminating spiking is a strong indicator against fold, saddle-node on invariant circle, and saddle homoclinic orbit bifurcations, since it occurs only during Andronov-Hopf and fold limit cycle bifurcations. However, large amplitude spiking cannot rule out Andronov-Hopf and fold limit cycle bifurcations, since many relaxation systems near (singular) Andronov-Hopf bifurcations exhibit small and intermediate amplitude spiking only in an extremely narrow region of bifurcation parameters; see e.g. Figs. 73 and 76.

\subsubsection{Damped oscillations at rest}

Existence of damped subthreshold oscillation is a strong indicator of nearness to an Andronov-Hopf 


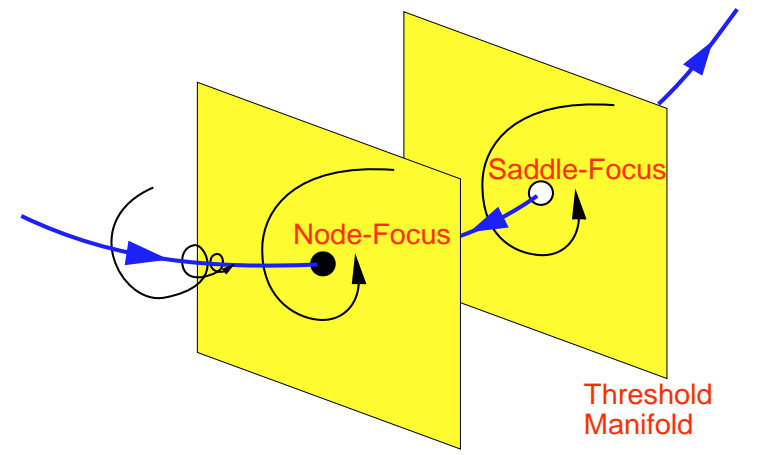

Fig. 122. A small perturbation may exhibit damped oscillation even though the system is near fold bifurcation point.

bifurcation. Its frequency is the neuron's resonant frequency. However, damped oscillations may exist even for fold bifurcation provided that the dimension of the system is greater than 2; see Fig. 122 .

Such an oscillation does not make the neuron a resonator, since it occurs in a subspace tangent to the stable manifold of the saddle-node. Increas- ing the amplitude of the oscillation does not change significantly the distance to the threshold manifold, and hence does not facilitate the spike.

\subsubsection{Spike undershoot}

Can the presence or lack of spike undershoot be used to distinguish different types of bursters? The answer is $N O$.

It is commonly believed that the undershoot occurs because the limit cycle attractor corresponding to the repetitive spiking encompasses the equilibrium corresponding to the quiescent state. This indeed leads to the undershoot, but only in a twodimensional fast subsystem. Moreover, the undershoot may occur without the encompassing, as we illustrate in Fig. 123. This suggests, in particular, that the famous "fold/homoclinic" ("squarewave" or "Type Ia") bursting may display undershoot even when the fast subsystem is planar.

If the fast system is multidimensional, then whether the limit cycle surrounds the equilibrium

\section{"Fold/Homoclinic" ("Square-Wave") Bursting}
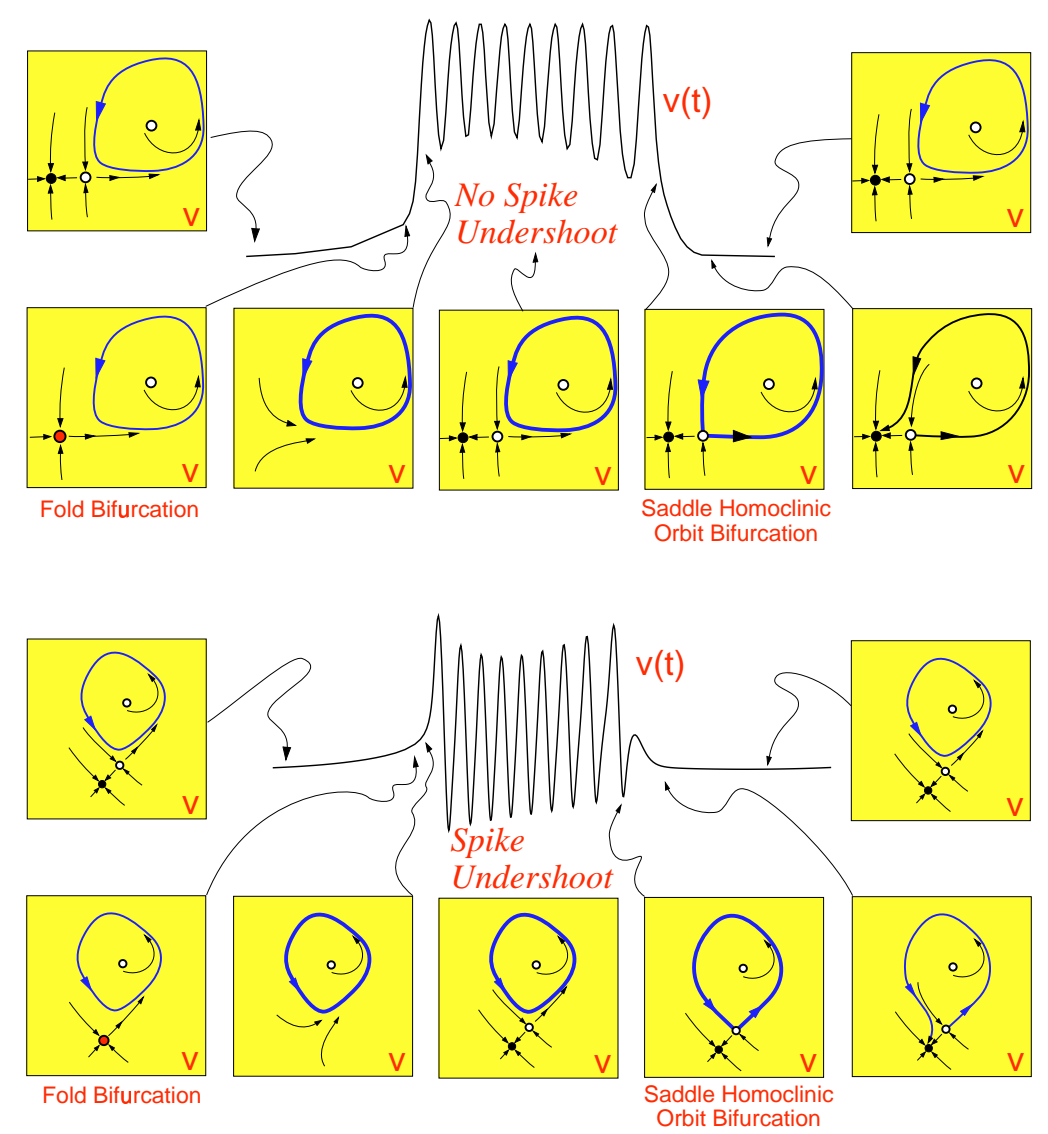

Fig. 123. Spike undershoot in "fold/homoclinic" ("square-wave" or "Type Ia") bursting. 


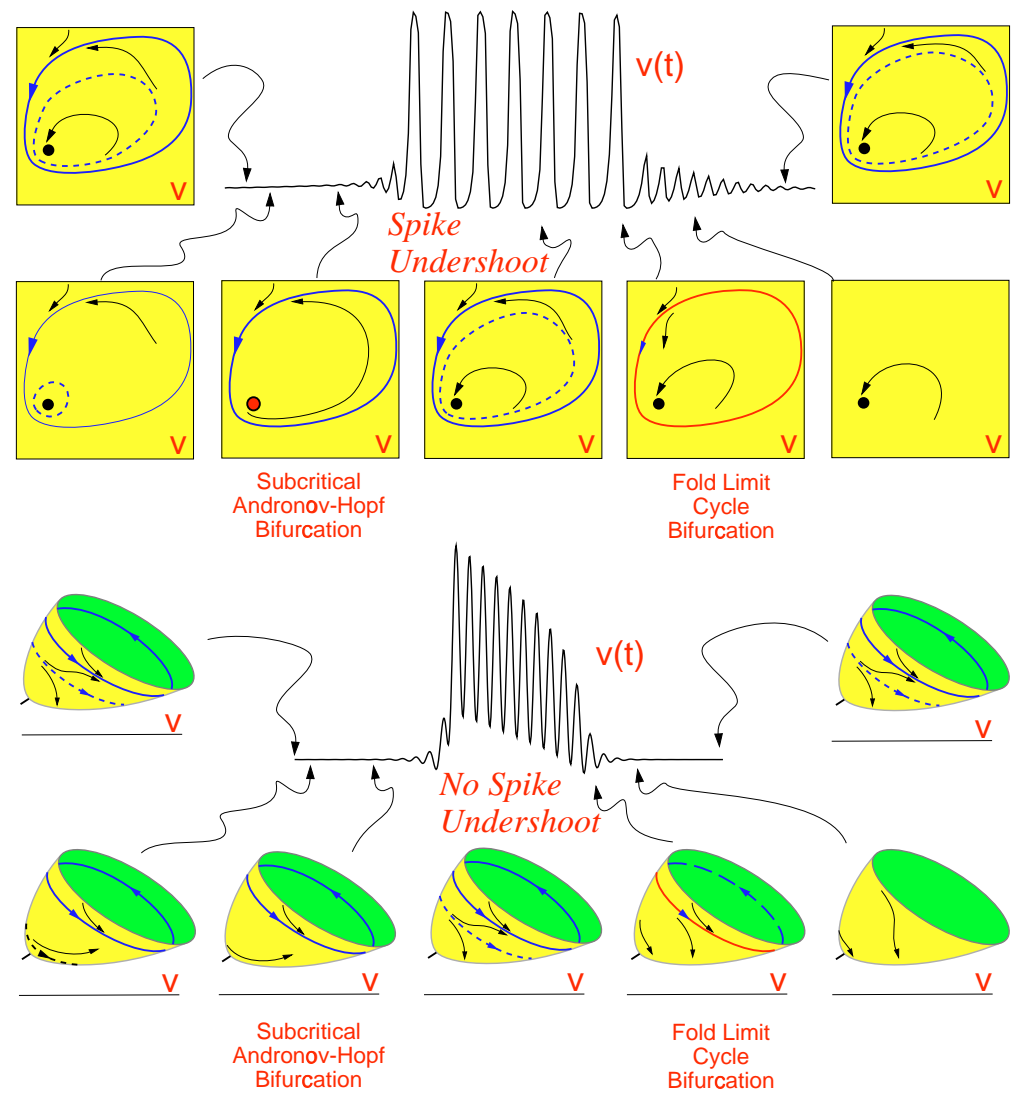

Fig. 124. The absence of spike undershoot in "subHopf/fold cycle" ("elliptic") burster when the fast subsystem is three-dimensional.

is irrelevant. Besides, the notion "surrounds" is not well defined in this case, since it depends on the point of view. In Fig. 124 we illustrate the issue and show that the "subHopf/fold cycle" bursting may not display undershoot.

Obviously, spike undershoot depends upon the overlapping of the orthogonal projections onto the voltage axis of the limit cycle attractor corresponding to repetitive spiking and the stable equilibrium corresponding to the quiescent state; see Fig. 125. Since the overlap depends exclusively on the location of the attractors, but not on the bifurcation mechanism leading to their appearance or disappearance, spike undershoot should not be used as an indicator of the type of neural bursting.

\subsubsection{Coexistence of spiking and quiescent states}

Global coexistence of attractors may not be useful in identifying class of bursting since any burster can have stable quiescent and spiking states. However, local coexistence may be useful.

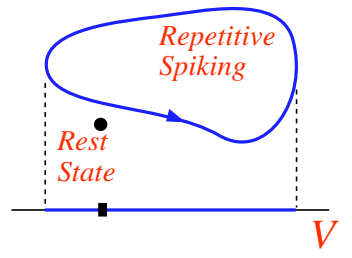

Spike Undershoot

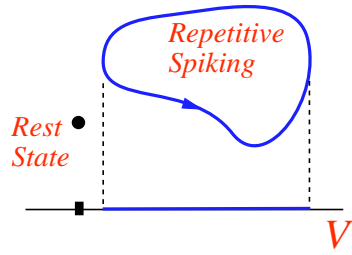

No Spike Undershoot
Fig. 125. Orthogonal projections of the limit cycle attractor and the stable equilibrium on the voltage axis $v$.

Let us elaborate. Consider the "circle/circle" bursting in Fig. 49 or 52. The spiking limit cycle attractor coexists with the rest state, hence a suitable short perturbation can shut down the spiking. The perturbation however should be strong enough to push the solution into the attraction domain of the down-state. In contrast, repetitive spiking in the "fold/homoclinic" bursting in Fig. 59 can be shut down prematurely by a weak perturbation having appropriate timing.

In general, repetitive spiking in any "*/homoclinic" and "*/fold cycle" bursting can be shut down 
prematurely by a weak stimulation, while that in "*/circle" and "*/Hopf" cannot. Similarly, a brief weak stimulation can evoke repetitive spiking in "fold/*" and "subHopf/*" bursters, and cannot in "circle/*" and "Hopf/*" bursters.

Finally, notice that testing for coexistence of attractors is an invasive procedure that requires stimulation, while the other criteria discussed above are noninvasive because they are based only on observations.

\subsubsection{Transition from bursting to tonic spiking or quiescence}

Fine structure of a bursting pattern depends on many physiological parameters, such as temperature, concentration of extracellular ions, etc. Changing such parameters can distort the bursting pattern, and in extreme cases, can shut it down completely or transform it to a tonic spiking. These are interesting cases because they correspond to a bifurcation in the behavior of the entire system. Measuring the interspike and/or interburst intervals at the bifurcation, one can in principle determine the type of bursting the system can exhibit. Unfortunately, little is known about possible mechanisms of transition from bursting to tonic spiking or quiescence, except in some special cases discussed by Terman [1992] and Izhikevich [2000a]. Thus, more analysis is needed before one can make sense of those measurements.

\subsubsection{Conductance based models}

We have treated neurons as general dynamical systems with no restriction on the form of the righthand side. Now we restrict ourselves and consider the conductance-based models of the form

$$
\begin{aligned}
\dot{V} & =I-\sum_{i=1}^{n} g_{i}(x)\left(V-E_{i}\right) \\
\dot{x} & =f(V, x)
\end{aligned}
$$

where $V$ is the voltage, and $x \in \mathbb{R}^{m}$ is the vector of conductances, gating variables, etc. Does the conductance based form impose any restriction on the possible bifurcations of the rest state or limit cycle? Could some bifurcation always lead to spike undershoot or overshoot? These are important open problems.

\section{Conclusion}

In this paper we review relevant bifurcations that are involved in the generation of action potentials by neurons. The bifurcations determine excitable properties of neurons, and hence their neurocomputational features, which are summarized in Tables 1 and 2. Table 4 classifies fast-slow bursters. Among them only a few, shown in Fig. 126, were identified before, the others are new.

Neurons: Integrators or resonators? The type of bifurcation of the rest state determines the most important neuro-computational feature of the neuron: It is either an integrator or a resonator.

- Integrator. If the rest state disappears via fold or saddle-node on invariant circle bifurcations, then the neuron acts as an integrator; the higher the frequency of the input, the sooner it fires.

- Resonator. If the rest state disappears via an Andronov-Hopf bifurcation, then the neuron acts as a resonator; it prefers a certain (resonant) frequency of the input spike train that is equal to a low-order multiple of its eigenfrequency. Increasing the frequency of the input may delay or even terminate its response.

Integrators have a well-defined threshold manifold, while resonators usually do not. Integrators distinguish between weak excitatory and inhibitory inputs, while resonators do not, since an inhibitory pulse can make a resonator fire. Integrators can easily encode information about the intensity of stimulation into their mean firing rate, whereas resonators cannot. In contrast, resonators are sensitive to the fine temporal structure of the input spike train, while integrators are not because they average (integrate) it. It is curious that many investigators tried to illustrate various aspects of sensitivity of biological neurons to timings of spikes, such as coincidence detection, using only the integrate-and-fire model.

Another astonishing fact is that many neurocomputational features of integrators, such as allor-none response and threshold manifold, have been introduced by studying the classical HodgkinHuxley model, which is a resonator, and hence does have these features.

Bursting. All bursting neurons seem to have similar behavior: Repetitive spiking, then quiescence, then spiking again, and so on. However, 


\begin{tabular}{|l|c|c|c|c|}
\hline Bifurcations & $\begin{array}{c}\text { Saddle-Node } \\
\text { on Invariant } \\
\text { Circle }\end{array}$ & $\begin{array}{c}\text { Saddle } \\
\text { Homoclinic } \\
\text { Orbit }\end{array}$ & $\begin{array}{c}\text { Supercritical } \\
\text { Andronov-Hopf }\end{array}$ & $\begin{array}{c}\text { Fold Limit } \\
\text { Cycle }\end{array}$ \\
\hline Fold & triangular & $\begin{array}{c}\text { square-wave } \\
\text { Type I }\end{array}$ & $\begin{array}{c}\text { tapered } \\
\text { Type V }\end{array}$ & Type IV \\
\hline $\begin{array}{l}\text { Saddle-Node on } \\
\text { Invariant Circle }\end{array}$ & $\begin{array}{c}\text { parabolic } \\
\text { Type II }\end{array}$ & & & \\
\hline $\begin{array}{l}\text { Supercritical } \\
\text { Andronov-Hopf }\end{array}$ & & & & elliptic \\
\hline $\begin{array}{l}\text { Subcritical } \\
\text { Andronov-Hopf }\end{array}$ & & & & Type III \\
\hline
\end{tabular}

Fig. 126. Bifurcation mechanisms of classical bursters. See also Tables 3 and 4 .

bifurcation analysis of the bursting mechanism reveals that seemingly similar bursters can have quite different neuro-computational properties. Some bursters act as integrators, others act as resonators. The former may exhibit burst synchronization, but could be reluctant to exhibit spike synchronization, while the latter could easily do both [Izhikevich, 2000a]. Thus, it is important to distinguish bursters.

Rigorous attempt to classify bursters started from the seminal paper by Rinzel [1987], and it was extended by other researchers. Bifurcation mechanisms of those classical bursters are summarized in Fig. 126. Since their naming scheme was awkward and frequently misleading, we faced a challenging problem to provide a novel and convenient nomenclature. We suggest to name bursters after the two fundamental bifurcations involved, see Fig. 2. The advantage of such a naming scheme is that it is selfexplanatory for most scientists.

Our classification of bursters is complete for codimension 1 planar fast-slow bursters described by smooth ODEs. Since there are only six relevant codimension 1 bifurcations of the quiescent state and four relevant codimension 1 bifurcations of the spiking state on a plane, there are only 24 planar fast-slow bursters, which are summarized in Table 3. Each of them can have subtypes depending on the type of the hysteresis loop (Fig. 55) and whether the quiescent state is inside or outside of the spiking limit cycle attractor.

The classification of nonplanar bursters may still be incomplete. Indeed, we took into account all known relevant bifurcations of codimension 1 , but there could be new bifurcations discovered in the future, which would lead to new nonplanar bursters. Besides, we did not consider bifurcations in piecewise smooth and delay systems. Finally, we provided a dozen or so examples of bursters of the hedgehog type, but we do not have any meaningful framework for their classification.

\section{Acknowledgments}

The author thanks Frank C. Hoppensteadt for his constant support and active collaboration in writing this paper. Special thanks to Alan R. Champneys, who noticed a missing bifurcation in the first draft of the paper, and to Richard Bertram, Cristina Soto-Trevino and Peter Rowat, who scrutinized the second draft and made many valuable suggestions. The author thanks Arthur Sherman for hospitality during his visit to the NIH where he learnt about point-point bursters. This research was supported by the NSF grant "Canonical Models for Mathematical Neuroscience", DMS-9805544.

\section{References}

Abarbanel, H. D. I., Huerta, R., Rabinovich, M. I., Rulkov, N. F., Rowat, P. F. \& Selverston, A. I. [1996] "Synchronized action of synaptically coupled chaotic model neurons," Neural Comput. 8, 1567-1602.

Alexander, J. C. \& Cai, D. [1991] "On the dynamics of bursting systems," J. Math. Biol. 29, 405-423.

Alexander, J. C., Doedel, E. J. \& Othmer, H. G. [1990] "On the resonance structure in a forced excitable system," SIAM J. Appl. Math. 50, 1373-1418.

Arnold, V. I. [1982] Geometrical Methods in the Theory of Ordinary Differential Equations (SpringerVerlag, NY); Russian original [1977] Additional 
Chapters of the Theory of Ordinary Differential Equations, Moscow.

Arnold, V. I., Afrajmovich, V. S., Il'yashenko, Yu. S. \& Shil'nikov, L. P. [1994] "Bifurcation theory," in $D y$ namical Systems V. Bifurcation Theory and Catastrophe Theory, ed. Arnold, V. I. (Springer-Verlag, NY).

Aronson, D. G., Ermentrout, G. B. \& Kopell, N. [1990] "Amplitude response of coupled oscillators," Physica D41, 403-449.

Baer, S. M., Erneux, T. \& Rinzel, J. [1989] "The slow passage through a Hopf bifurcation: Delay, memory effects, and resonances," SIAM J. Appl. Math. 49, $55-71$.

Baer, S. M., Rinzel, J. \& Carrillo, H. [1995] "Analysis of an autonomous phase model for neuronal parabolic bursting," J. Math. Biol. 33, 309-333.

Bedrov, Y. A., Akoev, G. N. \& Dick, O. E. [1992] "Partition of the Hodgkin-Huxley type model parameter space into regions of qualitatively different solutions," Biol. Cybern. 66, 413-418.

Belair, J. \& Holmes, P. [1984] "On linearly coupled relaxation oscillations," Quarterly of Appl. Math. 42, 193-219.

Bertram, R. [1993] "A computational study of the effects of serotonin on a molluscan burster neuron," Biol. Cybern. 69, 257-267.

Bertram, R., Butte, M. J., Kiemel, T. \& Sherman, A. [1995] "Topological and phenomenological classification of bursting oscillations," Bull. Math. Biol. 57, 413-439.

Booth, V., Carr, T. W. \& Erneux, T. [1997] "Nearthreshold bursting is delayed by a slow passage near a limit point," SIAM J. Appl. Math. 57, 1406-1420.

Butera Jr., R. J., Clark Jr., J. W. \& Byrne, J. H. [1996] "Dissection and reduction of a modeled bursting neuron," J. Comput. Neurosci. 3, 199-223.

Butera Jr., R. J., Clark Jr., J. W. \& Byrne, J. H. [1997] "Transient responses of a modeled bursting neuron: Analysis with equilibrium and averaged nullclines," Biol. Cybern. 77, 307-322.

Canavier, C. C., Clark, J. W. \& Byrne, J. H. [1991] "Simulation of the bursting activity of neuron-R15 in aplysia - role of ionic currents, calcium balance, and modulatory transmitters," J. Neurophysiol. 66, $2107-2124$.

Carpenter, G. A. [1979] "Bursting phenomena in excitable membranes," SIAM J. Appl. Math. 36, 334-372.

Chay, T. R. \& Keizer, J. [1983] "Minimal model for membrane oscillations in the pancreatic $\beta$-cell," Biophys. J. 42, 181-190.

Connor, J. A. \& Stevens, C. F. [1971] "Prediction of repetitive firing behavior from voltage-clamped data on an isolated neurone soma," J. Physiol. Lond. 214, $31-53$.

Del Negro, C. A., Hsiao, C.-F., Chandler, S. H. \&
Garfinkel, A. [1998] "Evidence for novel bursting mechanism in rodent trigeminal neurons," Biophys. J. 75, 174-182.

de Vries, G. [1998] "Multiple bifurcations in a polynomial model of bursting oscillations," J. Nonlin. Sci. 8, 281-316.

Ermentrout, G. B. [1996] "Type I membranes, phase resetting curves, and synchrony," Neural Comput. 8, 979-1001.

Ermentrout, G. B. [1998] "Linearization of F-I curves by adaptation," Neural Comput. 10, 1721-1729.

Ermentrout, G. B. \& Kopell, N. [1986a] "Parabolic bursting in an excitable system coupled with a slow oscillation," SIAM J. Appl. Math. 46, 233-253.

Ermentrout, G. B. \& Kopell, N. [1986b] "Subcellular oscillations and bursting," Math. Biosci. 78, 265-291.

Evans, J., Fenichel, N. \& Feroe, J. [1982] "Double impulse solutions in nerve axon equations," SIAM J. Appl. Math. 42, 219-234.

Fenichel, N. [1971] "Persistence and smoothness of invariant manifolds for flows," Ind. Univ. Math. J. 21, 193-225.

Feroe, J. A. [1982] "Existence and stability of multiple impulse solutions of a nerve equation," SIAM J. Appl. Math. 42, 235-246.

FitzHugh, R. [1955] "Mathematical models of threshold phenomena in the nerve membrane," Bull. Math. Biophys. 17, 257-278.

Frankel, P. \& Kiemel, T. [1993] "Relative phase behavior of two slowly coupled oscillators," SIAM J. Appl. Math. 53, 1436-1446.

Grasman, J. [1987] Asymptotic Methods for Relaxation Oscillations and Applications (Springer-Verlag, NY).

Guckenheimer, J., Harris-Warrick, R., Peck, J. \& Willms, A. [1997] "Bifurcations, bursting and spike frequency adaptation," J. Comput. Neurosci. 4, $257-277$.

Gutfreund, Y., Yarom, Y. \& Segev, I. [1995] "Subthreshold oscillations and resonant frequency in guinea-pig cortical neurons: Physiology and modeling," J. Physiol. London 483, 621-640.

Gutkin, B. S. \& Ermentrout, G. B. [1998] "Dynamics of membrane excitability determine interspike interval variability: A link between spike generation mechanisms and cortical spike train statistics," Neural Comput. 10, 1047-1065.

Hansel, D., Mato, G. \& Meunier, C. [1995] "Synchrony in excitatory neural networks," Neural Comput. 7, 307-335.

Hassard, B. D. [1978] "Bifurcation of periodic solutions of the Hodgkin-Huxley model for the squid giant axon," J. Theoret. Biol. 71, 401-420.

Hassard, B. D., Kazarinoff, N. D. \& Wan, Y. H. [1981] Theory and Applications of Hopf Bifurcation (Cambridge University Press, Cambridge).

Hastings, S. [1976] "On the existence of homoclinic 
and periodic orbits for FitzHugh-Nagumo equations," Quart. J. Math. (Oxford) 27, 123-134.

Hindmarsh, J. L. \& Rose, R. M. [1984] "A model of neuronal bursting using three coupled first order differential equations," Philos. Trans. R. Soc. London, Ser. B221 87-102.

Hodgkin, A. L. [1948] "The local electric changes associated with repetitive action in a non-medulated axon," J. Physiol. 107, 165-181.

Hodgkin, A. L. \& Huxley, A. F. [1952] "A quantitative description of membrane current and application to conduction and excitation in nerve," J. Physiol. 117, 500-544.

Holden, L. \& Erneux, T. [1993a] "Slow passage through a Hopf bifurcation: Form oscillatory to steady state solutions," SIAM J. Appl. Math. 53, 1045-1058.

Holden, L. \& Erneux, T. [1993b] "Understanding bursting oscillations as periodic slow passages through bifurcation and limit points," J. Math. Biol. 31, 351-365.

Holden, A. V., Hyde, J. \& Muhamad, M. [1991] "Equilibria. Periodicity, bursting and chaos in neural activity," Proc. 9th Summer Workshop on Mathematical Physics, Vol. 1, pp. 96-128.

Hoppensteadt, F. C. [1997] An Introduction to the Mathematics of Neurons. Modeling in the Frequency Domain (Cambridge University Press).

Hoppensteadt, F. C. [1993] Analysis and Simulations of Chaotic Systems (Springer-Verlag, NY).

Hoppensteadt, F. C. \& Izhikevich, E. M. [1996] "Synaptic organizations and dynamical properties of weakly connected neural oscillators: I. Analysis of canonical model," Biol. Cybern. 75, 117-127.

Hoppensteadt, F. C. \& Izhikevich, E. M. [1997] Weakly Connected Neural Networks (Springer-Verlag, NY).

Hoppensteadt, F. C. \& Izhikevich, E. M. [1998] "Thalamo-Cortical interactions modeled by weakly connected oscillators: Could brain use FM radio principles?" BioSyst. 48, 85-94.

Hutcheon, B., Miura, R. M. \& Puil, E. [1996] "Models of subthreshold membrane resonance in neocortical neurons," J. Neurophysiol. 76, 698-714.

Hutcheon, B., Miura, R. M., Yarom, Y. \& Puil, E. [1994] Low-threshold calcium current and resonance in thalamic neurons: A model of frequency preference," $J$. Neurophysiol. 71, 583-594.

Il'iashenko, Iu. S. \& Li, W. [1999] Nonlocal Bifurcations Mathematical Surveys and Monographs (American Mathematical Society), Vol. 66.

Izhikevich, E. M. [2001] "Resonate-and-fire neurons," Neural Networks, submitted.

Izhikevich, E. M. [2000a] "Subcritical elliptic bursting of Bautin type," SIAM J. Appl. Math. 60, 503-535.

Izhikevich, E. M. [2000b] "Phase equations for relaxation oscillators," SIAM J. Appl. Math., in press.

Izhikevich, E. M. [1999a] "Weakly connected quasiperi- odic oscillators, FM interactions, and multiplexing in the brain," SIAM J. Appl. Math. 59, 2193-2223.

Izhikevich, E. M. [1999b] "Class 1 neural excitability, conventional synapses, weakly connected networks, and mathematical foundations of pulse-coupled models," IEEE Trans. Neural Networks 10, 499-507.

Izhikevich, E. M. [1999c] "Weakly pulse-coupled oscillators, FM interactions, synchronization, and oscillatory associative memory," IEEE Trans. Neural Networks 10, 508-526.

Izhikevich, E. M. [1998] "Supercritical elliptic bursting, slow passage effect, and assistance of noise," preprint.

Jansen, H. \& Karnup, S. [1994] "A spectral analysis of the integration of artificial synaptic potentials in mammalian central neurons," Brain Res. 666, 9-20.

Johnston, D. \& Wu, S. M. [1995] Foundations of Cellular Neurophysiology (The MIT Press).

Kopell, N. [1995] "Chains of coupled oscillators," in Brain Theory and Neural Networks, ed. Arbib, M. A. (The MIT press, Cambridge, MA).

Kopell, N. \& Somers, D. [1995] "Anti-phase solutions in relaxation oscillators coupled through excitatory interactions," J. Math. Biol. 33, 261-280.

Kowalski, J. M., Albert, G. L., Rhoades, B. K. \& Gross, G. W. [1992] "Neuronal networks with spontaneous, correlated bursting activity: Theory and simulations," Neural Networks 5, 805-822.

Kuznetsov, Yu. [1995] Elements of Applied Bifurcation Theory 2nd edition (Springer-Verlag, NY).

Levi, M., Hoppensteadt, F. C. \& Miranker, W. L. [1978] "Dynamics of the Josephson junction," Quart. J. Appl. Math. July, 167-190.

Llinas, R. R. [1988] "The intrinsic electrophysiological properties of mammalian neurons: Insights into central nervous system function," Science $\mathbf{2 4 2}$, 1654-1664.

Llinas, R. R., Grace, A. A. \& Yarom, Y. [1991] "In vitro neurons in mammalian cortical layer 4 exhibit intrinsic oscillatory activity in the $10-$ to $50-\mathrm{Hz}$ frequency range," Proc. Natl. Acad. Sci. USA 88, 897-901.

Mishchenko, E. F., Kolesov, Yu. S., Kolesov, A. Yu. \& Rozov, N. K. [1994] Asymptotic Methods in Singularly Perturbed Systems (Plenum Press, NY).

Morris, C. \& Lecar, H. [1981] "Voltage oscillations in the Barnacle giant muscle fiber," Biophys. J. 35, 193-213.

Nejshtadt, A. [1985] "Asymptotic investigation of the loss of stability by an equilibrium as a pair of eigenvalues slowly cross the imaginary axis," Usp. Mat. Nauk 40, 190-191.

Pernarowski, M. [1994] "Fast subsystem bifurcations in a slowly varied Liénard system exhibiting bursting," SIAM J. Appl. Math. 54, 814-832.

Pernarowski, M., Miura, R. M. \& Kevorkian, J. [1992] "Perturbation techniques for models of bursting electrical activity in pancreatic $\beta$-cells," SIAM J. Appl. Math. 52, 1627-1650. 
Plant, R. E. [1981] "Bifurcation and resonance in a model for bursting nerve cells," J. Math. Biol. 11, 15-32.

Puil, E., Meiri, H., Yarom, Y. [1994] "Resonant behavior and frequency preference of thalamic neurons," $J$. Neurophysiol. 71, 575-582.

Rinzel, J. [1987] "A formal classification of bursting mechanisms in excitable systems," Mathematical Topics in Population Biology, Morphogenesis, and Neurosciences, eds. Teramoto, E. \& Yamaguti, M., Vol. 71 of Lecture Notes in Biomathematics (Springer-Verlag, Berlin).

Rinzel, J. \& Ermentrout, G. B. [1989] "Analysis of neural excitability and oscillations," eds. Koch, C. \& Segev, I. Methods in Neuronal Modeling (The MIT Press, Cambridge).

Rinzel, J. \& Lee, Y. S. [1986] "On different mechanisms for membrane potential bursting," Nonlinear Oscillations in Biology and Chemistry, ed. Othmer, H. G., Lecture Notes in Biomathematics (Springer-Verlag).

Rinzel, J. \& Lee, Y. S. [1987] "Dissection of a model for neuronal parabolic bursting," J. Math. Biol. 25, 653-675.

Rinzel, J. \& Miller, R. N. [1980] "Numerical calculation of stable and unstable periodic solution to the Hodgkin-Huxley equations," Math. Biosci. 49, 27-59.

Rush, M. E. \& Rinzel, J. [1995] "The potassium ACurrent, low firing rates and rebound excitation in Hodgkin-Huxley models," Bull. Math. Biol. 57, 899-929.

Rush, M. E. \& Rinzel, J. [1994] "Analysis of bursting in a thalamic neuron model," Biol. Cybern. 71, 281-291.

Samoilenko, A. M. [1991] "Elements of the mathematical theory of multi-frequency oscillations," Mathematics and Its Applications (Soviet Series), Vol. 71 (Kluwer Academic, Dordrecht).

Schecter, S. [1987] "The saddle-node separatrix-loop bifurcation," SIAM J. Math. Anal. 18, 1142-1156.

Sharp, A. A., O'neil, M. B., Abbott, L. F. \& Marder, E. [1993] "Dynamic clamp: Computer-generated conductances in real neurons," J. Neurophysiol. 69, 992-995.

Shepherd, G. M. [1981] "Introduction: The nerve impulse and the nature of nervous function," Neurones Without Impulses, eds. Roberts \& Bush (Cambridge University Press).

Shepherd, G. M. [1983] Neurobiology (Oxford University Press, NY).

Shorten, P. R. \& Wall, D. J. N. [2000] "A HodgkinHuxley model exhibiting bursting oscillations," Bull. Math. Biol., accepted.

Sivan, E., Segel, L. \& Parnas, H. [1995] "Modulated excitability: A new way to obtain bursting neurons," Biol. Cybern. 72, 455-461.

Smolen, P., Terman, D. \& Rinzel, J. [1993] "Properties of a bursting model with two slow inhibitory variables," SIAM J. Appl. Math. 53, 861-892.

Softky, W. R. \& Koch, C. [1993] "The highly irregu- lar firing of cortical-cells is inconsistent with temporal integration of random EPSPs," J. Neurosci. 13, 334-350.

Somers, D. \& Kopell, N. [1993] "Rapid synchronization through fast threshold modulation," Biol. Cybern. 68, 393-407.

Somers, D. \& Kopell, N. [1995] "Waves and synchrony in networks of oscillators or relaxation and nonrelaxation type," Physica D89, 169-183.

Soto-Trevino, C., Kopell, N. \& Watson, D. [1996] "Parabolic bursting revisited," J. Math. Biol. 35, 114-128.

Storti, D. W. \& Rand, R. H. [1986] "Dynamics of two strongly coupled relaxation oscillators," SIAM J. Appl. Math. 46, 56-67.

Taylor, D. \& Holmes, P. [1998] "Simple models for excitable and oscillatory neural networks," J. Math. Biol. 37, 419-446.

Terman, D. [1991] "Chaotic spikes arising from a model of bursting in excitable membranes," SIAM J. Appl. Math. 51, 1418-1450.

Terman, D. [1992] "The transition from bursting to continuous spiking in excitable membrane models," $J$. Nonlinear Sci. 2, 133-182.

Terman, D. \& Lee, E. [1997] "Partial synchronization in a network of neural oscillators," SIAM J. Appl. Math. 57, 252-293.

Terman, D. \& Wang, D. [1995] "Global competition and local cooperation in a network of neural oscillators," Physica D81, 148-176.

Traub, R. D. \& Miles, R. [1991] Neuronal Networks of the Hippocampus (Cambridge University Press, Cambridge).

Troy, W. [1978] "The bifurcation of periodic solutions in the Hodgkin-Huxley equations," Quart. Appl. Math. 36, 73-83.

Wang, X.-J. [1993] "Ionic basis for intrinsic $40 \mathrm{~Hz}$ neuronal oscillations," NeuroReport 5, 221-224.

Wang, X.-J. [1993] "Genesis of bursting oscillations in the Hindmarsh-Rose model and homoclinicity to a chaotic saddle," Physica D62, 263-274.

Wang, X.-J. [1998] "Calcium coding and adaptive temporal computation in cortical pyramidal neurons," $J$. Neurophysiol. 79, 1549-1566.

Wang, X. J. \& Rinzel, J. [1995] "Oscillatory and bursting properties of neurons," Brain Theory and Neural Networks, ed. Arbib, M. A. (The MIT press, Cambridge, MA).

Williams, T. L. \& Sigvardt, K. A. [1995] "Spinal cord of lamprey: Generation of locomotor patterns," Brain Theory and Neural Networks, ed. Arbib, M.A. (The MIT press, Cambridge, MA).

Wilson, C. J. [1993] "The generation of natural firing patterns in neostriatal neurons," Progress in Brain Research, eds. Arbuthnott, G. W. \& Emson, P. C. 99, pp. 277-297. 
Wilson, C. J. \& Kawaguchi, Y. [1996] "The origins of two-state spontaneous membrane potential fluctuations of neostriatal spiny neurons," J. Neurosci. 16, $2397-2410$.

Wilson, H. R. \& Cowan, J. D. [1972] "Excitatory and inhibitory interaction in localized populations of model neurons," Biophys J. 12, 1-24.
Wilson, M. A. \& Bower, J. M. [1989] "The simulation of large scale neural networks," Methods in Neuronal Modeling, eds. Koch, C. \& Segev, I. (The MIT Press, Cambridge, MA).

Wu, H.-Y. \& Baer, S. M. [1998] "Analysis of an excitable dendritic spine with an activity-dependent stem conductance," J. Math. Biol. 36, 569-592. 